Portland State University

PDXScholar

$1-1-2011$

\title{
Synthesis and Characterization of Glyconanomaterials, and Their Applications in Studying Carbohydrate-Lectin Interactions
}

Xin Wang

Portland State University

Follow this and additional works at: https://pdxscholar.library.pdx.edu/open_access_etds Let us know how access to this document benefits you.

Recommended Citation

Wang, Xin, "Synthesis and Characterization of Glyconanomaterials, and Their Applications in Studying Carbohydrate-Lectin Interactions" (2011). Dissertations and Theses. Paper 626.

https://doi.org/10.15760/etd.626

This Dissertation is brought to you for free and open access. It has been accepted for inclusion in Dissertations and Theses by an authorized administrator of PDXScholar. Please contact us if we can make this document more accessible: pdxscholar@pdx.edu. 
Synthesis and Characterization of Glyconanomaterials, and Their Applications in Studying Carbohydrate-Lectin Interactions

by

Xin Wang

A dissertation submitted in partial fulfillment of the requirements for the degree of

\author{
Doctor of Philosophy \\ in \\ Chemistry
}
Dissertation Committee:
Mingdi Yan, Chair
Andrea M. Goforth
Andres H. La Rosa
Shankar B. Rananavare
Jun Jiao

Portland State University

(C) 2011 


\begin{abstract}
This dissertation focuses on the synthesis and characterization of glyconanomaterials, as well as their applications in studying carbohydrate-protein interactions. A new and versatile method for coupling underivatized carbohydrates to nanomaterials including gold and silica nanoparticles was developed via the photochemically induced coupling reaction of perfluorophenylazide (PFPA). A wide range of carbohydrates including mono-, oligo- and poly-saccharides were conjugated to the nanoparticles with high yields and efficiency. New analytical methods were developed to determine the binding affinities of glyconanoparticles (GNPs) with lectins; these include fluorescence-based competition assay, dynamic light scattering (DLS) and isothermal titration calorimetry (ITC). Results showed that the multivalent presentation of carbohydrate ligands significantly enhanced the binding affinity of GNPs by several orders of magnitude compared to the free ligands. Systematic studies were carried out to investigate the impact of ligand presentation, i.e., the type and length of spacer linkage, the ligand density and the nanoparticle size on the binding affinity of the resulting glyconanoparticles. We used gold GNPs to study interactions with anti-HIV lectin cyanovirin-N (CV-N), and dye-doped silica nanoparticles for labeling glyans and developing high-throughput screening technique.
\end{abstract}


Acknowledgements

I wish to express my warm and sincere thanks to my advisor Prof. Mingdi Yan. Her broad knowledge and logical way of thinking have been of great value for me. The present dissertation would not have been possible without her understanding, encouraging and personal supervision.

I want to thank my committee members: Prof. Andrea M. Goforth, Prof. Andres H. La Rosa, Prof. Shankar B. Rananavare and Prof. Jun Jiao for their generous guidance.

I would like to express special thanks to Prof. Olof Ramström from Department of Chemistry at KTH- Royal Institute of Technology, Sweden, visiting professors Prof. Raymond Bard (University of Portland) and Prof. Takuya Kubo (Tohoku University, Japan) for their kind efforts on providing valuable suggestions and help.

I would like thank my past and current labmates: Dr. Lihong Liu, Dr. Hui Wang, Dr. Ke Jiang, Dr. Anuradha Tyagi, Suji Uppalapati, Sailaja Chada, Kai Wang, Qi Tong, Liling Li, Surangi Jayawardena, Jing Yuwen and Xuan Chen. It is a pleasure to work with them, and discuss about our research. All of my co-workers offered me lots of help, as well as valuable suggestions to my works. I also want to thank Lingquan Deng (KTH), Dr. Matei and Dr. Koharudin (U. Pitts) for providing samples for my studies.

I owe my loving thanks to my wife Xiaohua Wang and my parents. Without their encouragement and great helps it would have been impossible for me to finish this work. 
Table of Contents

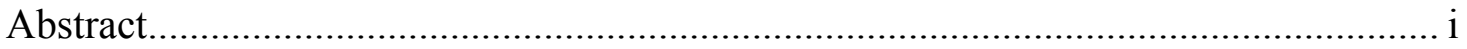

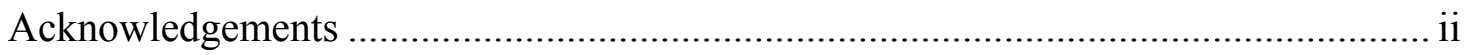

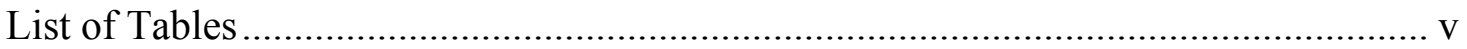

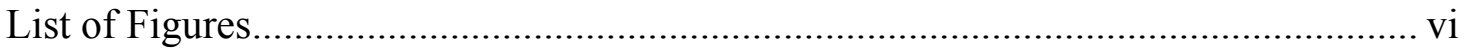

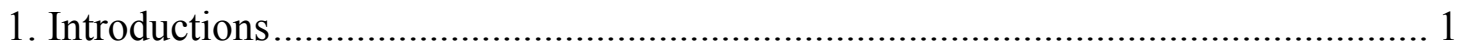

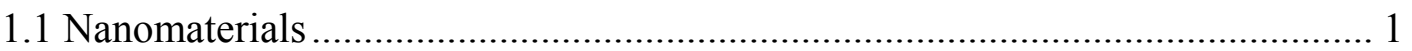

1.2 Nanomaterials surface functionalization .................................................. 6

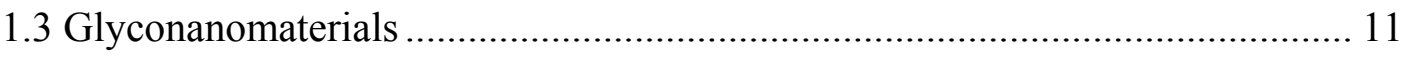

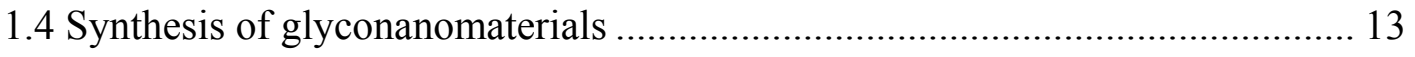

2. Development of a Photocoupling Method for the Synthesis of Glyconanoparticles

3. Quantitative Analysis of Binding Affinity of Glyconanoparticles with Lectins ...... 44

3.1 Fluorescence-based competition assays ................................................. 48

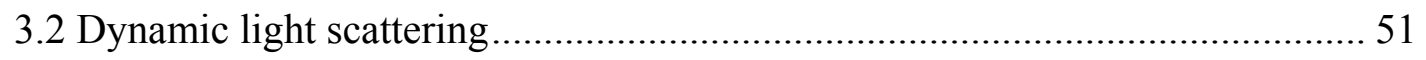

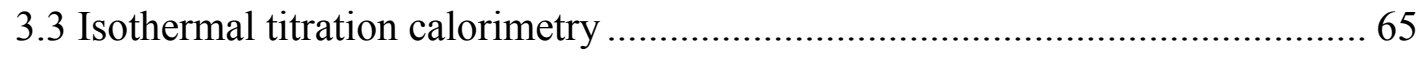

4. Impact of Ligand Presentation on the Binding Affinity of Glyconanoparticles-

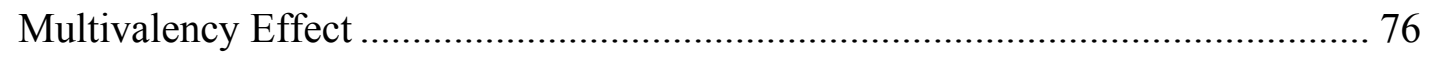

5. Applications of Glyconanomaterials in Bioanalysis ...................................... 105

5.1 Signal enhancement study glycan-lectin interactions using anti-HIV lectins. 106 
5.2 Labeling native glycans with dye-doped silica nanoparticles ...................... 119

5.3 High-throughput glycan screening on lectin microarray ............................ 136

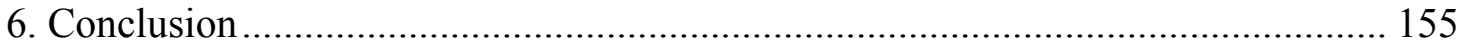

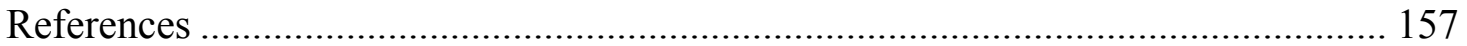

Appendices

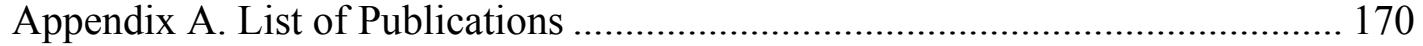

Appendix B. Copyright Information....................................................... 172 


\section{List of Tables}

Table 1.1 Properties of typical nanomaterials and biomedical applications 3

Table 1.2 Typical complementary functional groups of covalent coupling chemistries 10

Table 2.1 Coupling efficiency and surface coverage of D-mannose immobilized on Au NPs using PFPA-disulfide 2 as the coupling agent 32

Table 3.1 LOD and $\mathrm{K}_{\mathrm{D}}$ values of GNP-lectin interactions. 60

Table 3.2 The thermodynamic parameters of GNP-lectin interactions at $25^{\circ} \mathrm{C}$ 74

Table 4.1 Coupling yield of mono-, oligo-, and poly-saccharides on Au NPs............ 88

Table 4.2 Binding affinity of different GNPs with Con A ..................................... 94

Table 4.3 Binding affinity as a function of ligand density .................................... 96

Table 4.4 Binding affinity of GNPs with Con A .................................................. 100

Table 4.5 Binding affinity of GNPs of varying sizes ........................................... 102

Table 5.1 Affinities for Man2/3 $\left(\mathrm{K}_{\mathrm{d}}\right)$ and GNP-M2/3 $\left(\mathrm{K}_{\mathrm{D}}\right)$ binding to $\mathrm{CVN}^{\mathrm{Q} 50 \mathrm{C}} \ldots \ldots 117$

Table 5.2 Ligand density and coupling yield of glyco FSNPs ............................... 135

Table 5.3 Lectins and their corresponding carbohydrate binding ligands................ 145

Table 5.4 Association constants $\left(\mathrm{K}_{\mathrm{a}}\right)$ of lectins and glycans................................ 148

Table 5.5 Apparent $\mathrm{K}_{\mathrm{D}}$ values of glyco-FSNPs with lectins obtained from supermicroarrays 152 


\section{List of Figures}

Figure 1.1. Modification of nanomaterials surface by non-covalent and covalent

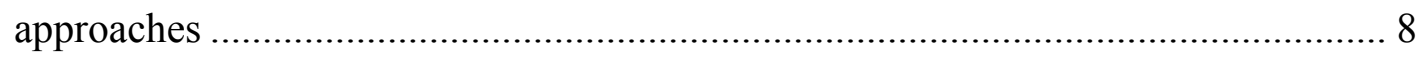

Figure 2.1 Simplified description of phenylazide photochemistry. ......................... 19

Figure 2.2 Synthesis of PFPA-Au NPs and subsequent coupling of $\alpha$-1,4-mannobiose...

Figure 2.3 Synthesis of PFPA disulfides 1 and 2.

Figure 2.4 (a) UV-vis spectra (insert: Au nanoparticle solutions) b) TEM micrographs (scale bar: $200 \mathrm{~nm}$ ) of Au NPs (A), PFPA-disulfide-functionalized Au NPs (B), Au NPs with surface-coupled $\alpha-1,4$-mannobiose (C), and subsequent treatment with Con A (D). c) Schematic illustration of the interaction of mannobiose-coupled $\mathrm{Au}$ NPs with Con A, and the formation of Au NP aggregates. 30

Figure 2.5 UV-vis spectra of (a) monosaccharide-, and (b) disaccharide-functionalized $\mathrm{Au}$ NPs before and after binding with Con $\mathrm{A}$ 34

Figure 2.6 SPR peak shifts of carbohydrate-functionalized Au NPs after treating with various lectins 36

Figure $2.7{ }^{1} \mathrm{H}$ NMR spectra of PFPA-disulfide 1 and gold nanoparticles functionalized with PFPA-disulfide 1 (Au-PFPA) in $\mathrm{CDCl}_{3}$ with TMS (0.0 ppm). 38

Figure 2.8 FT-IR spectra of PFPA-disulfide 1, gold nanoparticles functionalized with PFPA-disulfide 1 (Au-PFPA), and Au NPs subsequently coupled with $\alpha-1,4-$ mannobiose (Au-DiMan)...... 
Figure 2.9 Calibration curve obtained by treating various concentrations of D-mannose with anthrone/sulfuric acid and measuring the absorption at $620 \mathrm{~nm}$.

Figure 2.10 D-mannose molecules and Au NPs. 41

Figure 2.11 UV-vis spectra of PFPA-functionalized Au NPs and after treating with $100 \mathrm{nM}$ and $1000 \mathrm{nM}$ Con A.

Figure 3.1. Equilibria involved in the competition binding assay (left). Concentration dependent fluorescence intensity curve (right)

Figure 3.2 (a) Synthesis of silica and Au GNPs: S-GNP and Au-GNP. (b) GNP aggregation upon addition of lectin 56

Figure 3.3 (a-d) DLS spectra and TEM images (inserts, scale bars: $50 \mathrm{~nm}$ ) when 35 nm S-M-GNP was treated with varying concentrations of Con A. (e) The particle size vs. concentration of Con A.

Figure 3.4 The change in particle diameter $(\Delta \mathrm{D})$ vs. lectin concentration: experimental data (circles) and the corresponding Hill fitting curves (lines) for a) $35 \mathrm{~nm}, \mathrm{~b}) 110$ nm, and $470 \mathrm{~nm}$ silica NPs, respectively. The scale bars in the TEM images are a) $50 \mathrm{~nm}$, b) $200 \mathrm{~nm}$, and c) $500 \mathrm{~nm}$ respectively. 59

Figure 3.5 Percent increase in particle size (= increase in particle diameter $(\Delta \mathrm{D})$ /original particle diameter $\left.\left(\mathrm{D}_{0}\right) \times 100 \%\right)$ vs. concentration of Con $\mathrm{A}$ for various GNPs 61

Figure 3.6 (a) Diagram of ITC cells and (b) Typical ITC data. Top: raw ITC data.

Bottom: binding isotherm curve. 65 
Figure 3.7 ITC graphs of GNP-Man with a) Con A and b) PNA. The experimental data (solid squares) were fit to theoretical titration curves (solid lines) using the software supplied by the ITC manufacturer 69

Figure 3.8 ITC titration graphs of Con A with (a) GNP-Man2I, (b) GNP-Man2II, and (c) GNP-Man2III. Solid squares: experimental data, lines: fitted titration curves.. 71 Figure 3.9 ITC titration graphs of GNP-Man2 with CV-N Mut ${ }^{\mathrm{DB}}$ 73

Figure 4.1 Synthesis of PFPA-thiols a-d. 79

Figure 4.2 Functionalization of Au NPs with PFPA-thiol and subsequent coupling of carbohydrates.

Figure 4.3 a) UV-vis spectra of AuNP-a-Man upon addition of increasing concentration of Con A. (b) Absorbance of GNPs at $650 \mathrm{~nm}$ vs. Con A concentration

Figure 4.4 (a) The fluorescence-based competition binding assay; (b) two equilibriums established in the system; (c) Cheng-Prusoff equation, where $\mathrm{IC}_{50}=$ concentration of ligand displaying $50 \%$ of specific binding; $[\mathrm{M}]=$ concentration of free ligand, $\mathrm{K}_{\mathrm{d} 1}=$ dissociation constant of the free ligand with Con $\mathrm{A}$; and $\mathrm{K}_{\mathrm{d} 2}=$ dissociation constant of GNPs with Con A; (d) fluorescence spectra of the supernatant as a function of increasing concentration of AuNP-b-Man; (e) concentration response curve, where the $\mathrm{IC}_{50}$ value was obtained.

Figure 4.5 Binding affinity vs. ligand density for Man (a), Man2 (b), and Man3 (c) with Con A 
Figure 4.6 TEM images of gold nanoparticles in 4 different sizes: $7 \mathrm{~nm}$ (a), $14 \mathrm{~nm}$ (b), $22 \mathrm{~nm}(\mathrm{c})$ and $30 \mathrm{~nm}(\mathrm{~d})$ 101

Figure 4.7 Schematic illustration of Con A and GNP 103

Figure 5.1 Synthesis of Man2- and Man3-conjugated AuNPs GNP-M2 and GNPM3.

Figure 5.2 UV-vis spectra of GNP-M3 before (solid line) and after (dotted line) treatment with a) $\mathrm{CVN}^{\mathrm{Q} 50 \mathrm{C}}$ and b) $\mathrm{CVN}^{\mathrm{MutDB}}$. TEM micrographs of GNP-M3 treated with c) $\mathrm{CVN}^{\mathrm{Q} 50 \mathrm{C}}$ and d) $\mathrm{CVN}^{\mathrm{MutDB}}$ 113

Figure 5.3 Fluorescence competition assay. a) Schematic representation of binding scenario. b) Modified Cheng-Prusoff equation based on a competitive two site binding model, where $[\mathrm{M}]$ is the concentration of the free ligand, and $\mathrm{K}_{\mathrm{d} 1}$ and $\mathrm{K}_{\mathrm{d} 2}$ are the dissociation constants of the free ligand for the glycan-binding sites on Domains $\mathrm{A}$ and $\mathrm{B}$ of $\mathrm{CVN}^{\mathrm{Q} 50 \mathrm{C}}$, respectively. The data were fitted using the maximum bound fractions, $\mathrm{f}_{\mathrm{B} \max 1}$ and $\mathrm{f}_{\mathrm{Bmax} 2}$, corresponding to the two binding sites, and dissociation constants $\mathrm{K}_{\mathrm{D} 1}$ and $\mathrm{K}_{\mathrm{D} 2}$ as adjustable parameters. c) Concentration response curves of GNP-M2 and GNP-M3

Figure 5.4 Calorimetric titration of a) $\mathrm{CVN}^{\mathrm{Q} 50 \mathrm{C}}(35 \mu \mathrm{M})$ with Man2 $(1.5 \mathrm{mM})$, and b) $\mathrm{CVN}^{\mathrm{Q} 50 \mathrm{C}}(50 \mu \mathrm{M})$ with Man3 $(0.64 \mathrm{mM})$ at $30^{\circ} \mathrm{C}$. The raw data were obtained for 30 and 20 automatic injections, respectively. The integrated curves show experimental points (-) and the best fit (-). The buffer was $50 \mathrm{mM}$ sodium phosphate, $200 \mathrm{mM} \mathrm{NaCl}, \mathrm{pH} 7.5$, containing $0.02 \% \mathrm{NaN}_{3}$ 116 
Figure 5.5 (a) Synthesis of PFPA-functionalized FSNPs, and (b) high-throughput synthesis of glyco FSNPs

Figure 5.6 Fluorescence spectra of FITC-doped silica nanoparticles before and after

UV irradiation for $10 \mathrm{~min}$

Figure 5.7 TEM images after FSNP-Man was treated with E. coli strain ORN178 (a), or ORN208 (b). Insert: fluorescence image of the corresponding sample 129

Figure 5.8 (a) Preparation of lectin microarray, incubation with FSNP-Man, and fluorescence imaging. (b) Fluorescence image and (c) fluorescence intensities of lectin microarray after treating with FSNP-Man. 130

Figure 5.9 TEM image of FITC-doped SNPs 133

Figure 5.10 Size distribution of FITC-doped SNPs measured by DLS 133

Figure 5.11 Fluorescence spectra of FITC-doped silica nanoparticles before and after UV irradiation for $10 \mathrm{~min}$ 134

Figure 5.12 Fluorescence spectra of FITC before and after UV irradiation for 10 $\min$ 134

Figure 5.13 Schematics of lectin super-microarray fabrication, and the subsequent assays with glyco-FSNPs.

Figure 5.14 Schematics of (a) high-throughput screening, and (b) competition assay on super microarrays. 138

Figure 5.15 Interactions of Man2-FSNPs with super-microarray: (a) fluorescence intensity vs. the printing concentration of Con A ( $\square$ ); (b) fluorescence intensity vs. 
the concentration of Man2 -FSNPs (o); (c) fluorescence intensity vs. Man2-FSNPs $(1.5 \mathrm{mg} / \mathrm{mL})$ incubation time (inserts: fluorescence images of Con A spots (top panel) and SBA spots (bottom panel)). The concentration of Man2-FSNPs in (a) was $1.5 \mathrm{mg} / \mathrm{mL}$, and the printing concentration of Con A was $1 \mathrm{mg} / \mathrm{mL}$ in (b)... 143 Figure 5.16 Fluorescence (a) and AFM images (b-d) of a printed Con A spot (1 $\mathrm{mg} / \mathrm{mL})$ after incubation with Man2-FSNP $(1.5 \mathrm{mg} / \mathrm{mL})$ for $2 \mathrm{~h}$.......................... 143

Figure 5.17 Carbohydrate structures used in this study ......................................... 146

Figure 5.18 Fluorescence image (a, c) and fluorescence intensity (b, d) of lectin supermicroarrays interacting with glyco-FSNPs

Figure 5.19 (a) Fluorescence image of a lectin super-microarray after incubating with Man2-FSNP and varying concentrations of $2 \alpha-M a n 2 . ~(b-d)$ Fluorescence intensities vs. free $2 \alpha-$ Man2 concentration for CVN-Q CVN-M (c) and Con A (d) 151 


\section{Chapter 1. Introduction}

\subsection{Nanomaterials}

Research on nanoscience and nanotechnology has increased exponentially over the past decade, with new investigations and discoveries appearing daily ${ }^{1}$. Unique properties of optical, electronic, magnetic, mechanical, and chemical reactivities have been discovered and are associated with nanomaterials solely because of their nanoscale sizes and shapes. These materials serve as model systems providing fundamental understanding of structure-property relationships at the nanoscale. The investigations in turn guide the creation of new structures, systems, and devices with novel properties, functions, and utilities. The interdisciplinary research on nanomaterials merges the fields of synthetic and materials chemistry, condense-matter physics, and fabrication engineering, solving problems in materials synthesis and characterization, and providing core frameworks for biomedical functions ${ }^{2}$. While challenges remain in improving the capabilities of characterization tools at the nanoscale, and the synthesis of nanomaterials of well-defined size, shape and composition, progress has already been made beyond fundamental research to the development of diverse and versatile nanomaterials-based biomedical devices adopting nanomaterials into current biomedical technologies ${ }^{3,4}$. For instance, chipbased microfluidic nanodevices enable high-throughput and exceptionally efficient analysis of gene sequences, greatly expanding the ability for the characterization of 
genetic makeup and revolutionalizing the specificity of diagnostics and therapeutics. Nanomaterials, having at least one dimension smaller than $100 \mathrm{~nm}$, are comparable in size to many biological molecules. The nanosize dimension allows them to incorporate into cells for in vitro and in vivo imaging, drug-delivery, and targeting tumor cells ${ }^{5}$. Nanosensors aid early detection and prevention of diseases, and nanodevices, used remotely and in vivo, show high promise for effective and low-cost home-based health-care, benefiting the well-being of the entire human society.

The field of nanomaterials is vastly diverse and is evolving rapidly. A great variety of nanomaterials are synthesized for biomedical applications (Table 1.1); a considerable number of which are polymeric nanomaterials formed either by applying the techniques of nanofabrication or via molecular self-assembly. These polymers, carrying multiple or multifunctional ligands by synthesis or physical encapsulation, have been used in biomedical imaging, as vehicles for drug delivery, and as scaffolds in tissue engineering ${ }^{6}$. The functionalities on these polymeric nanomaterials are in general built into the material synthesis rather than by surface functionalization. 
Table 1.1. Properties of typical nanomaterials and their biomedical applications

\begin{tabular}{|c|c|c|c|}
\hline \multicolumn{2}{|c|}{ Nanomaterials } & \multirow{2}{*}{ Intrinsic properties } & \multirow{2}{*}{ Biomedical applications } \\
\hline Category & Examples & & \\
\hline Metallic & $\mathrm{Au}, \mathrm{Ag}$ & SPR & $\begin{array}{l}\text { Biosensing, drug } \\
\text { delivery, bioimaging }\end{array}$ \\
\hline $\begin{array}{l}\text { Semiconduct } \\
\text { or }\end{array}$ & $\begin{array}{l}\mathrm{CdS}, \\
\mathrm{CdSe}\end{array}$ & $\begin{array}{l}\text { Fluorescence, } \\
\text { luminescence }\end{array}$ & $\begin{array}{l}\text { Immunoassays, } \\
\text { bioimaging, biosensing }\end{array}$ \\
\hline Magnetic & $\mathrm{Fe}_{3} \mathrm{O}_{4}$ & Magnetism & MRI, drug delivery \\
\hline $\begin{array}{l}\text { Carbon- } \\
\text { based }\end{array}$ & $\begin{array}{l}\text { CNTs, } \\
\text { Fullerene }\end{array}$ & $\begin{array}{l}\text { Electronic and mechanical } \\
\text { properties, conductivity }\end{array}$ & $\begin{array}{l}\text { Drug and gene delivery, } \\
\text { therapy, biosensing }\end{array}$ \\
\hline
\end{tabular}

Metal nanoparticles (NPs) such as Au and Ag NPs are excellent nanomaterials providing a powerful platform in biomedical applications of biomolecular recognition and sensing, drug delivery, and imaging ${ }^{7,8}$. Au NPs are among the mostly used and studied nanomaterials owing to their ease of preparation, stability, well-established surface functionalization chemistry, and their unique optoelectronic properties. The so-called surface plasmon resonance (SPR) absorption, produced by the collective oscillation of conducting electrons in the metal NP core upon interacting with the incident light, is dependent on the NP size and shape, the dielectric property of the media, and the distance between particles. This provides a unique and convenient platform for monitoring the molecular recognition event occurring at close to the 
surface of the nanoparticles. Colorimetric bioassays have thus been achieved based on the SPR shift when molecular interactions take place at the surface of the nanoparticles, and have been employed to study fundamental biorecognition processes including cell-cell communication, enzymatic activity, protein-protein interaction, and DNA hybridization. When the ligand-receptor interaction causes additional aggregation of nanoparticles, very large SPR shifts occur producing intense color changes visible to the naked eyes ${ }^{9}$. These optical properties, induced by single particles or interactions between particles, allow the highly sensitive detection of molecular binding events. In addition, the SPR absorptions are not subject to quenching/photobleaching that are frequently associated with organic fluorophores, or blinking that occurs in quantum dots. An early example was demonstrated by Mirkin and coworkers using oligonucleotide-capped Au NPs ${ }^{10}$. Hybridization of the complimentary oligonucleotide strands induced the aggregation of Au NPs leading to a distinct solution color change easily visualized by naked eyes. Numerous examples can be found in the literature where bioconjugated Au NPs are used as colorimetric biosensors detecting proteins, viruses, and bacteria at an extremely sensitive level. An additional advantage of NPs is that the multiple ligands presented on the NP surface could drastically enhance affinities of specific monovalent interactions via the multivalent binding between NPs and the biological target. Lin et al. reported that the observed binding affinity of mannose-encapsulated Au NPs with Concanavalin A (Con A) was several orders of magnitude higher in comparison with that of mannose with Con $A$ in solution ${ }^{11}$. In the study of Melander et al., SDC-1721, which is a 
structural fragment of the HIV inhibitor TAK-77 and displays no inhibition activity in solution, became a potent inhibitor when coupled to 2-nm Au NPs ${ }^{12}$. The authors attributed the enhanced activity to the multivalency effect where multiple ligands presented on the nanoparticle surface greatly enhanced the overall binding affinity with the protein.

Besides metallic nanoparticles, quantum dots (QDs) are zero-dimension materials exhibiting quantum confinement in all three spatial dimensions. QDs have broad excitation spectra yet narrow and tunable emissions, and have thus been widely used as optical labels in a wide range of biomedical applications including immunoassays for proteins, nucleic acids, bacteria and toxin analysis ${ }^{13,14}$. Compared with the organic fluorescent dyes, QDs have additional advantages of high quantum yields and high photochemical stability, and offer improved detection sensitivity and application lifetime ${ }^{15}$. Magnetic nanoparticles of iron oxides is another type of attractive nanomaterials that have a long history of investigation and have shown remarkable potentials in biomedical research, ${ }^{16-24}$ including magnetic resonance imaging (MRI) contrast enhancement ${ }^{25-27}$, drug delivery ${ }^{28-31}$, hyperthermia ${ }^{32-34}$, cell separation ${ }^{35-38}$, and tissue repair ${ }^{39}$. Superparamagnetic iron oxide nanoparticles can furthermore improve the diagnostic value by enhancing the MRI contrast on surrounding healthy and pathological tissues, increasing the MRI resolution at the microscopic-level ${ }^{40,41}$. 
An inherent feature of nanomaterials is their high surface areas, ie, high surface-tovolume ratio in comparison with their bulk material counterpart. For instance, for a CdSe QD of $\sim 2 \mathrm{~nm}$ in diameter, $\sim 90 \%$ of the atoms are located on the surface ${ }^{42}$. Nanomaterials thus have high surface energy resulting from increased surface curvature and a greater percentage of dangling bonds that lack nearest bonding neighbors. To minimize the surface energy, nanomaterials tend to adopt a spherical shape, and in addition, to agglomerate into large particles reducing the surface area and thus lowering the surface energy. Surface modification/passivation of nanomaterials is highly necessary where the surface layer serves to reduce the surface energy and at the same time acts as the protective coating preventing nanoparticles

from agglomerating thus increasing their long-term stability ${ }^{43}$. The capping layer can be further derivatized with additional ligands or functional groups introducing diverse functions and properties to the nanomaterials ${ }^{44}$.

\subsection{Nanomaterial Surface Functionalization}

Bio-functions and bio-compatibility of nanomaterials are realized by introducing synthetic ligands or natural biomolecules onto nanomaterials, and combining ligandreceptor biological interactions with intrinsic nanomaterial properties. Common strategies of engineering nanomaterial surfaces involve physisorption or chemisorption of desired ligands. Surface modification of nanomaterials follows the general strategies of non-covalent and covalent approaches (Figure 1.1). The non- 
covalent approach is a physisorption process where the ligand is adsorbed to the nanomaterials via the non-covalent forces, including electrostatic interactions, hydrogen bonding, and hydrophobic interactions. A popular method of non-covalent surface functionalization is the so-called steric stablization that involves polymers or surfactants as the capping layer. The surface coating stabilizes individual nanoparticles, and at the same time, the steric repulsion inhibits agglomeration by keeping the nanoparticle dispersion intact. An added benefit of this process is that monodisperse nanoparticles can be synthesized. The polymer layer adsorbed on the surface of nanoparticles serves as a diffusion barrier to the growing species, resulting in a diffusion-limited growth in the subsequent growth of nuclei. Diffusion-limited growth would reduce the size distribution of the initial nuclei, leading to monodisperse nanoparticles. Furthermore, polymers as the coating materials provide high-density functional groups that can be subsequently derivatized with appropriate ligands for bioconjugation. In the example by Star et. al, a FET device was constructed using polymer-coated carbon nanotubes (CNTs) for the detection of protein binding ${ }^{45}$. CNTs were coated with a mixture of poly(ethylene imine) (PEI) and poly(ethylene glycol) (PEG). PEI provided the functional groups, ie, $-\mathrm{NH}_{2}$, for the covalent immobilization of the ligand, biotin, to CNTs. PEG, on the other hand, served as a non-fouling coating preventing the non-specific adsorption of proteins on the device, thus giving much increased sensitivity. 


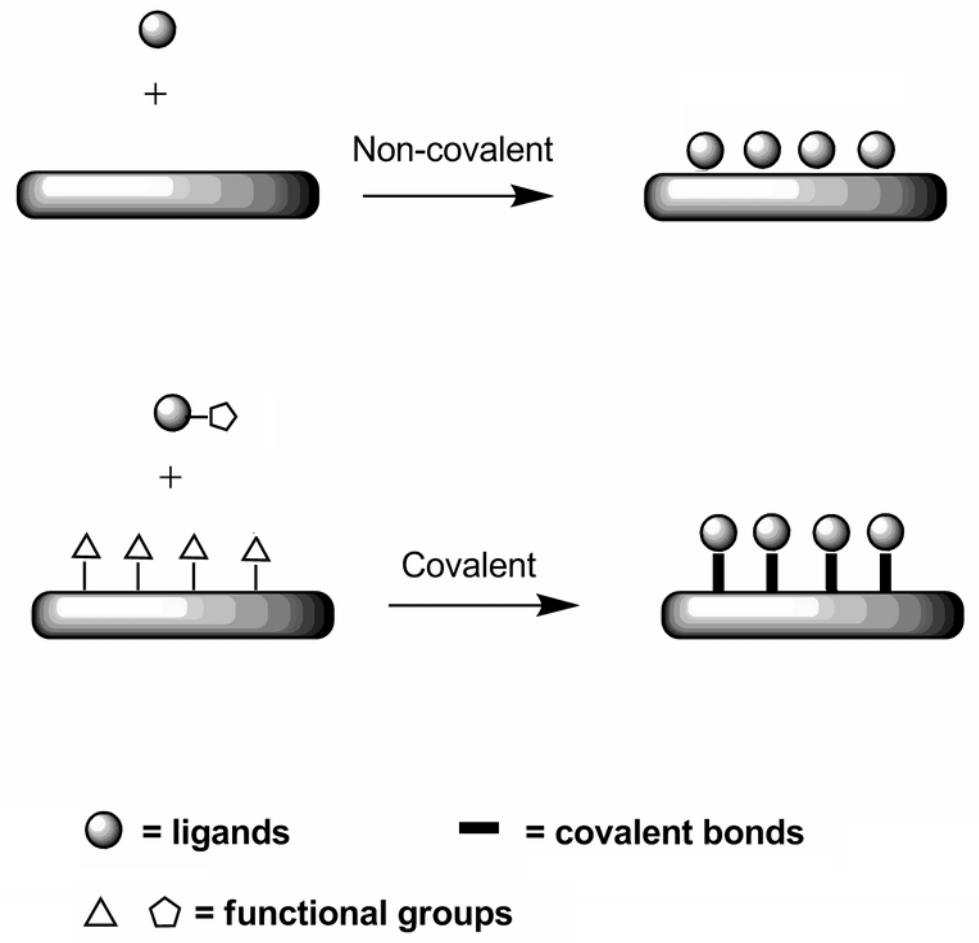

Figure 1.1. Modification of nanomaterial surface by non-covalent and covalent approaches.

The majority of surface functionalization methods are based on the covalent bond formation, which offers the advantage of robust linkage and the stability of the surface ligand. If the ligand possesses a functional group that is reactive towards the substrate materials, it can chemisorb to the nanomaterial surface and yield self-assembled structures. Typical examples of chemisorption include thiol/disulfide on metals (Au, $\mathrm{Ag}, \mathrm{Cu})$ and semiconductors $(\mathrm{CdS}, \mathrm{CdSe}, \mathrm{ZnS})$, silanes on oxides $\left(\mathrm{SiO}_{2}, \mathrm{TiO}_{2}\right)$, and phosphates on metal oxides (iron oxide, $\mathrm{TiO}_{2}$ ). Depending on the nature of the substrate material, ligands possessing the corresponding functional groups are chosen and synthesized. Of the chemisorbed self-assembly systems, thiol/Au is the most 
studied and used. The process is well-established and it produces well-behaved selfassembled monolayers that are stable, reproducible, and thoroughly characterized. The system is therefore widely used especially for proof-of-principle studies. The surface functionalization generally follows a simple solution process where the nanomaterial is immersed in a solution containing the ligand. The reaction occurs readily at room temperature. Excess ligands are then removed by rinsing with the solvent leaving behind nanomaterials that are surface-functionalized with the ligand. A highly effective surface engineering strategy is the so-called ligand exchange technique where the ligand of interest displaces the stabilizing capping layer on the nanomaterial. In this case the ligand should have at least equal or higher affinity than the capping molecule towards the nanomateiral in order to partially or fully displace them. In the synthesis of gold nanoparticles by the classic citrate reduction reaction of auric acid, the as-prepared Au NPs bear the citrate capping layer that can be subsequently replaced by a thiol or disulfide ligand ${ }^{46}$.

Covalent bond formation is also accomplished by reacting the complementary functional groups on the ligand and the surface of the nanomaterial. In this case, the nanomaterial is derivatized with a functional group, which then reacts with the ligand that either possesses the functional group in its native form or is derivatized by chemical synthesis. Table 1.2 shows typical complimentary functional groups used for coupling ligands to nanomaterials ${ }^{47-56}$. 
Table 1.2. Typical complementary functional groups of covalent coupling chemistries. (L: ligand)

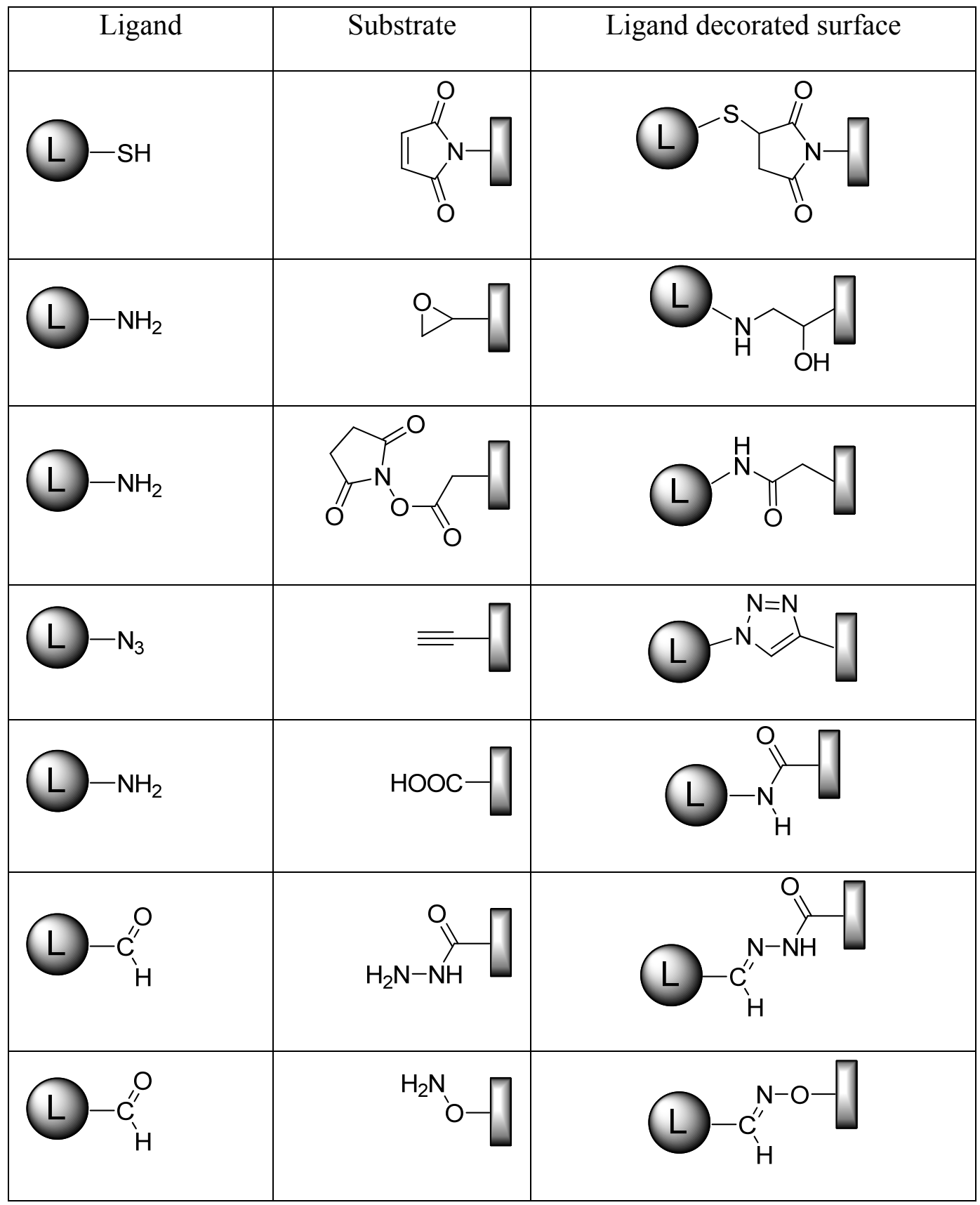




\subsection{Glyconanomaterials}

Carbohydrates are the most abundant biomolecules in nature and essential elements in a wide range of processes in living systems. Besides their use as structural materials and energy sources, they are to large extents mediating recognition events through their interactions with proteins and other biological entities. Complex carbohydrate structures are thus involved in, for example, cell communication and trafficking, tumor genesis and progression, immune responses, fertilization, apoptosis, and infection. ${ }^{57-63}$ Many challenges are, however, associated with the study of these processes, and the development of glycoscience has been largely hampered by the complexity and low abundance of the glycan structures involved, and the weak affinities often associated with carbohydrate-protein interactions. The field has recently experienced a dramatic upsurge, much on account of the very strong developments in carbohydrate synthesis, glycan analysis methods, and nanotechnology. ${ }^{64}$ New synthetic methods, such as automated strategies and enzymemediated protocols, have resulted in increased availability of complex carbohydrate structures promoting advances in the entire field. ${ }^{65-67}$

An important development in the field of glycoscience is the discovery that in biological systems, carbohydrates bind lectins, i.e., carbohydrate-binding proteins, in a highly cooperative manner to improve the weak affinity of individual carbohydrate ligands to the lectin. ${ }^{68-70}$ This cluster or multivalency effect involves multiple 
carbohydrate ligands and lectins interacting with each other enhancing binding affinity by several orders of magnitude. For example, oligosaccharides exhibit higher binding affinity than monosaccharides towards the same lectin. In the biological system, lectins associate with cells by interacting with the multiple copies of carbohydrate ligands on the cell surface, exhibiting binding affinities significantly higher than those of the interactions between the lectins and the isolated carbohydrate ligands. Although the quantitative aspect of the multivalency effect is yet to be established, the fact that multivalency can significantly enhance binding affinity has helped fuel a renewed interest in fundamental glycoscience and glycomaterial development. Extensive work has been conducted for conjugating carbohydrates to the scaffolds of proteins, peptides, lipids, and synthetic polymers. ${ }^{71}$ Synthetic strategies are applied to control the number of ligands on the scaffold, the spatial display of the ligands, and the structure of the scaffold, which in turn impact the binding affinity of the resulting glycoconjugates with their binding partners. These synthetic multivalent glycoconjugates could bind to receptors competitively having the potential to serve as inhibitors displacing natural ligands in the applications of carbohydrate-based drug design and therapeutics. ${ }^{72}$ When the scaffold is a flat solid surface, efficient glycan microarrays can be generated facilitating the development of high-throughput analysis of ligand-protein interactions in applications of ligand screening and diagnostics. ${ }^{73-80}$ Nanomaterials as scaffolds for carbohydrate ligand display have recently emerged, and glyconanomaterials have thus been synthesized, demonstrating great potential in biomedical imaging, diagnostics, and therapeutics. Compared with molecular scaffolds, 
nanomaterials as ligand carriers offer a number of attractive features. Nanomaterials, being small in size, have high specific surface areas and can therefore accommodate high-density ligands promoting multivalent interactions with their binding partners. The ligand density can be modulated by the size and shape of the nanomaterial, and multiple epitopes of the same ligand can be exposed and presented in a threedimensional format. Nanomaterials possess unique optical, electronic, magnetic, and mechanical properties, as well as chemical reactivities. These properties, together with their nanosized dimensions allow them to be incorporated into cells for in vitro and in vivo imaging, drug-delivery, and targeting tumor cells. This opens up a wide range of possibilities, the potential of which is just emerging. ${ }^{81-85}$

\subsection{Synthesis of glyconanomaterials}

Two general strategies for nanomaterial functionalization can be discerned, based on either non-covalent or covalent protocols. Both approaches are associated with advantages and drawbacks, although covalent protocols are generally preferred due to the considerably higher stabilities of the constructs.

\section{1) Non-covalent attachment}

A variety of glyconanomaterials based on physisorption of carbohydrate ligands to the material surface has been reported. The attachment relies on non-covalent interactions, 
including, for example, hydrogen bonding, Coulombic interactions, and hydrophobic effects. A method for producing metallic glyconanoparticles through electrostatic adsorption was reported by Yang et al., in which metal-chitosan nanocomposites were prepared on a range of different metals, including $\mathrm{Au}, \mathrm{Ag}, \mathrm{Pt}$ and $\mathrm{Pd} .^{86} \mathrm{The}$ nanoparticles were synthesized by reducing metal salts in the presence of chitosan, resulting in simultaneous ligand adsorption. Rosenzweig et al. synthesized dextrancoated quantum dots (QDs) where negatively-charged carboxymethyldextran was adsorbed onto QDs by mixing with positively-charged polylysine via electrostatic interactions. ${ }^{87}$ As noticed from these examples, a notable advantage of the physisorption strategy is that the reaction conditions are relatively mild, and minimal chemical derivatization is required for the nanomaterials substrates and the carbohydrate ligands. Nevertheless, the physical adsorption is relatively random and disordered compared to covalent linkages. In addition, the strength of the association is not sufficiently strong, which may lead to potential bond breakage during interactions, as well as increased nonspecific or unexpected interactions with the target molecules. This can significantly affect the specificity and sensitivity in applications such as biological sensing and recognition. However, as demonstrated in the mentioned examples, oligomer/polymer-based ligands can to some extent circumvent the stability problems.

\section{2) Covalent attachment}


The most commonly used method for conjugating carbohydrate structures to nanomaterials is based on covalent attachment. Among the various nanomaterials, $\mathrm{Au}$ NPs are the most extensively used scaffold materials especially in fundamental studies due to their ease of preparation, exceptional stability, and high reproducibility. ${ }^{88} \mathrm{Au}$ NPs of different sizes, shapes, and controlled dispersity can now be synthesized using simple solution-based methods. The well-established thiol- and disulfide-Au chemistry, first applied to nanoparticles using a two-phase system by Brust et al., allows the preparation of Au NPs with well-defined surfaces. ${ }^{89}$ These surface ligands serve as a protective layer to provide high stability for the nanomaterials in media ranging from organic solvents to biological milieus. The chemistry has been widely adopted to prepare Au NPs modified with various functional groups, and biological molecules including DNA, proteins, peptides, and carbohydrates have all been successfully introduced into the system. ${ }^{10,90,91}$ Penadés et al. reported the first synthesis of carbohydrate-functionalized Au NPs (glyconanoparticles, GNPs). ${ }^{92,93}$ The trisaccharide determinant of the Lewis ${ }^{\mathrm{x}}\left(\operatorname{Le}^{\mathrm{x}}\right)$ antigen was derivatized with an alkylthiol, and $\mathrm{Le}^{\mathrm{x}}$-coated $\mathrm{Au}$ NPs were prepared by reducing $\mathrm{HAuCl}_{4}$ with $\mathrm{NaBH}_{4}$ in presence of the thiol-derivatized Le ${ }^{\mathrm{x}}$. Based on this strategy, Au NPs functionalized with monosaccharides (glucose), disaccharides (maltose), and tetrasaccharides (Le ${ }^{\mathrm{y}}$ ) were prepared and applied to the studies of various biological interactions. ${ }^{94,95}$ Later, several other groups utilized a similar strategy to produce $\mathrm{Au}$ and $\mathrm{Ag}$ glyconanoparticles using thiolated carbohydrates. ${ }^{11,96-100}$ Furthermore, thiolated carbohydrate derivatives have been adopted in the preparation of glyco-quantum dots 
(GQDs). ${ }^{101-103}$ Additional coupling methods based on the reaction of complementary functional groups have also been developed to facilitate the conjugation of carbohydrates other than the thiolated derivatives. Examples include coupling $N$ hydroxysuccinimide (NHS)-functionalized dextran to amine-functionalized Ag NPs, ${ }^{104}$ and amine-derivatized carbohydrates to aldehyde-functionalized Au NPs. ${ }^{105}$ Copyright (2009) Royal Society of Medicine Press, UK

Overall, the objective of my $\mathrm{PhD}$ project is as follows:

1. To synthesize glyconanomaterials using the photocoupling strategy to covalently attach a variety of underivatized carbohydrates onto nanomaterials;

2. To develop new analytical methods to study the binding affinity of glyconanomaterials with lectins;

3. To comprehensively investigate the effect of ligand presentation on binding affinity on glyconanomaterials;

3. To develop applications of glyconanomaterials in bioanalysis, such as lectin recognition, affinity enhancement, glycan labeling, and high-throughput screening.

These studies will provide a fundamental understanding of glyconanomaterials, and illustrate their importance in studying carbohydrate-involved biological interactions. The methodologies established should benefit the study of bio-functionalized nanomaterials and enable a wide range of biomedical applications. 


\section{Chapter 2. Development of a Photocoupling Method for the Synthesis of}

\section{Glyconanoparticles}

This work was published on "Wang, X.; Ramström, O.; Yan, M., A Photochemically

Initiated Chemistry for Coupling Underivatized Carbohydrates to Gold Nanoparticles.

J. Mater. Chem. 2009, 19 (47), 8944-8949"

Reproduced by permission of The Royal Society of Chemistry 
Carbohydrate structures expressed on cell surfaces are vastly diverse. The matter is further complicated by the fact that half of all cellular proteins are post-translationally modified by the addition of structurally diverse and complex glycans with many types of chemical bonds and branch-chains. Therefore highly desirable for carbohydrate immobilization are effective coupling chemistries that are general and versatile, can accommodate ligand diversity and give stable interfaces, and yet are simple and reproducible. Current coupling methods involve chemical derivatization of carbohydrate structures to introduce functionality that then react with the functional groups on the nanoparticles, for example, by chemisorption of thiol-functionalized carbohydrates on gold nanoparticles.

In this chapter, a new technique has been developed and described for coupling underivatized carbohydrates on gold nanoparticles. The method is based on the photochemistry of perfluorophenylazides (PFPA), which upon light activation, undergoes $\mathrm{C}-\mathrm{H}$ insertion reaction with neighboring molecules. Phenylazides and derivatives were the most popular photoaffnity labeling (PAL) agents, due to their high reaction efficiency, fast kinetics, excellent storage stability and ease of synthesis. Upon light irradiation, they decompose by releasing $\mathrm{N}_{2}$ to give singlet phenylnitrene, a highly reactive intermediate which can undergo numerous nonselective reactions yielding a wide range of products. Three main processes of phenylnitrene reactions are shown in Figure 2.1: ring expansion, insertion/addition and intersystem crossing (ISC). ${ }^{106}$ 


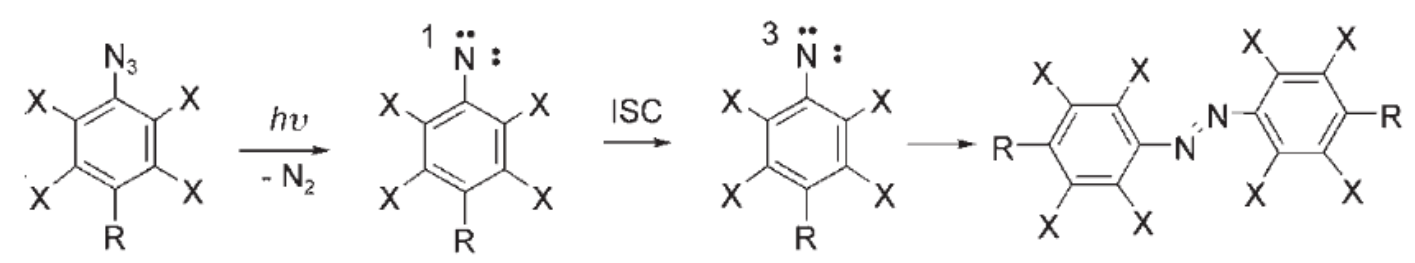

$$
X=H, F
$$
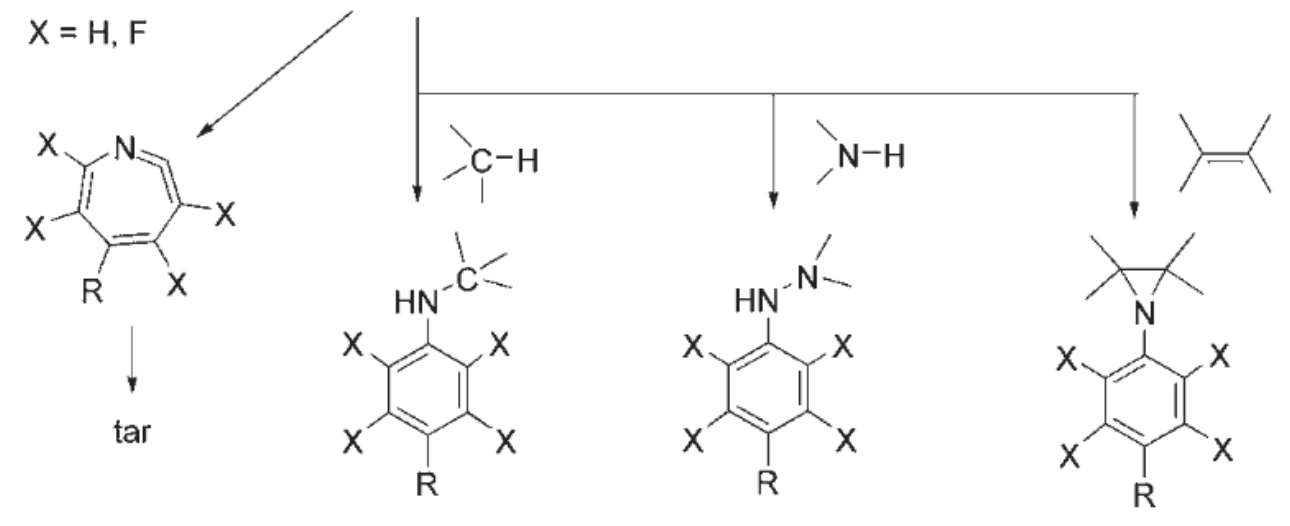

Figure 2.1 Simplified description of phenylazide photochemistry.

Because all carbohydrates and glycoconjugates possess $\mathrm{C}-\mathrm{H}$ bonds, no chemical derivatization is required and carbohydrate structures can be directly coupled to solid substrates in their native forms. We have successfully attached monosaccharides and oligosaccharides on Au nanoparticles using this approach. The immobilized carbohydrates retained their recognition abilities with lectins, and the binding strength, measured by the SPR red-shift of Au nanoparticles, was consistent with the binding affinity of the free carbohydrate with the corresponding lectin. This general coupling chemistry together with the unique and sensitive optical property of the resulting nanoparticles serve as a label-free and rapid detection platform readily applicable to clinical diagnosis, sensing, and ligand screening. 
Metal nanoparticles coupled with biological ligands have been widely used for monitoring activities of biomolecules and their interactions with ligands. ${ }^{107}$ A unique characteristic of metal nanoparticles as the recognition probe is their remarkable and tunable optical property, the so-called surface plasmon resonance (SPR), determined by their size and shape, the dielectric property of the media, and the distance between particles. Colorimetric bioassays have thus been achieved based on the SPR shift when molecular interactions take place at the surface of the nanoparticles, and have been employed to study fundamental biorecognition processes including cell-cell communication, enzymatic activity, protein-protein interaction, and DNA hybridization. ${ }^{9,108,109}$ When the ligand-receptor interaction causes additional aggregation of nanoparticles, significant red-shift of the SPR absorption occurs producing intense color changes visible to the naked eye. ${ }^{110-113}$

Naturally occurring carbohydrates, glycoproteins, and glycolipids are present at the surface of nearly every cell in living systems, and play crucial roles in biological events as recognition sites between cells and different binding partners. They for example mediate various phenomena including cell growth, inflammatory responses or viral infections, and changes in glycosylation are often involved in disease states, including cancer. Efficient analysis and control of such events are of high importance. Carbohydrate-based detection platforms have recently emerged as highly useful analytical and diagnostic tools, and have demonstrated tremendous potential to superior sensitivity, selectivity, and stability. ${ }^{68,114,115}$ A key technology requirement in 
receptor/ligand-based sensing and detection is the surface conjugation chemistry that can effectively couple ligands to solid substrates. Carbohydrate structures expressed on cell surfaces are vastly diverse and complex. Despite the development of new synthetic and enzymatic protocols, obtaining large quantities of glycans with the required functionality and precise glycosylation pattern is still a major challenge. Highly desirable are effective coupling chemistries that are general and versatile, can accommodate carbohydrate diversity and give stable interfaces, and yet are simple and reproducible. Ligands attached to solid surfaces through a covalent bond are more stable than those that are physisorbed by weaker forces. Of the reported covalent coupling chemistry, the most popular involves chemisorption of thiolated carbohydrates on Au nanoparticles. ${ }^{11,105,116-120}$ Other conjugation methods include coupling $N$-hydroxysuccinimide (NHS)-functionalized dextran to aminefunctionalized Ag NPs, ${ }^{104}$ and amine-functionalized carbohydrates to aldehydefunctionalized Au NPs. ${ }^{105}$ Coupling chemistry that does not require chemical derivatization of the carbohydrates is appealing. A few examples have been reported to conjugate underivatized carbohydrates on flat substrates for microarray construction, although the protocols have not been adapted for Au NPs. One approach used hydrazide-modified gold substrates where the hydrazide reacted with the terminal aldehyde group of the carbohydrates ${ }^{56,121}$ A similar approach employed aminefunctionalized surfaces and the coupling with carbohydrates took place by reductive amination to yield an amine conjugate. ${ }^{122}$ In both cases, reducing carbohydrates are 
necessary, and for monosaccharides, the coupled products often became acyclic and lost their binding affinity.

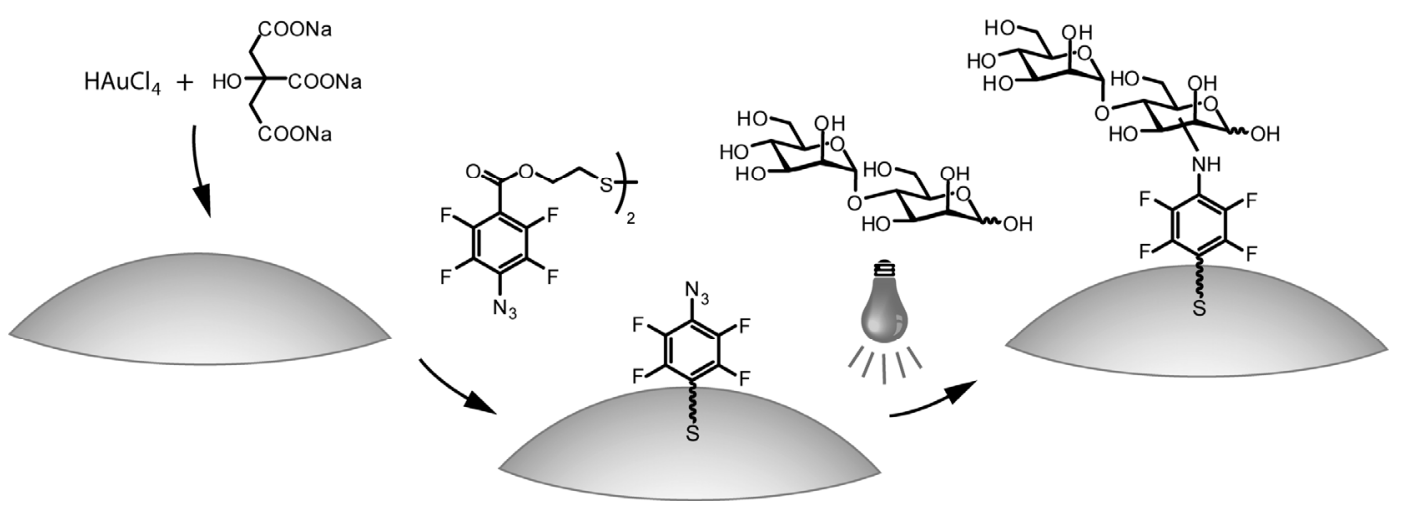

Figure 2.2 Synthesis of PFPA-Au NPs and subsequent coupling of $\alpha$-1,4-mannobiose.

We have successfully employed PFPAs in surface modification, targeting polymeric materials that lack reactive functional groups for surface coupling. ${ }^{123-126}$ Carbohydrates are another class of compounds that are well-suited for the PFPA photocoupling chemistry. The design of our approach is to prepare PFPAfunctionalized Au NPs which can be subsequently be used to covalently couple, in principle, any carbohydrate structures by way of the insertion reactions of photochemically activated nitrene species. (Figure 2.2) This coupling chemistry does not require chemical derivatization of the carbohydrate, which can be complex when multiple protection and glycosylation reactions are involved. PFPA has been successfully utilized to immobilize hyaluronan on polystyrene (PS) beads. ${ }^{127}$ PS beads with surface amino groups were treated with NHS-functionalized PFPA, and 
hyaluronan was then attached to the bead surface by UV irradiation. In this chapter, we report that PFPAs can be employed to conjugate monosaccharides and oligosaccharides on Au NPs. The surface-bound carbohydrates retained their binding affinity with the corresponding lectins, and the ranking of binding affinity was consistent with that observed for free ligands in solution. A colorimetry method was developed to determine the density of the carbohydrates attached to Au NPs. Results showed that the coupling chemistry is efficient and high yielding.

\section{Experimental details}
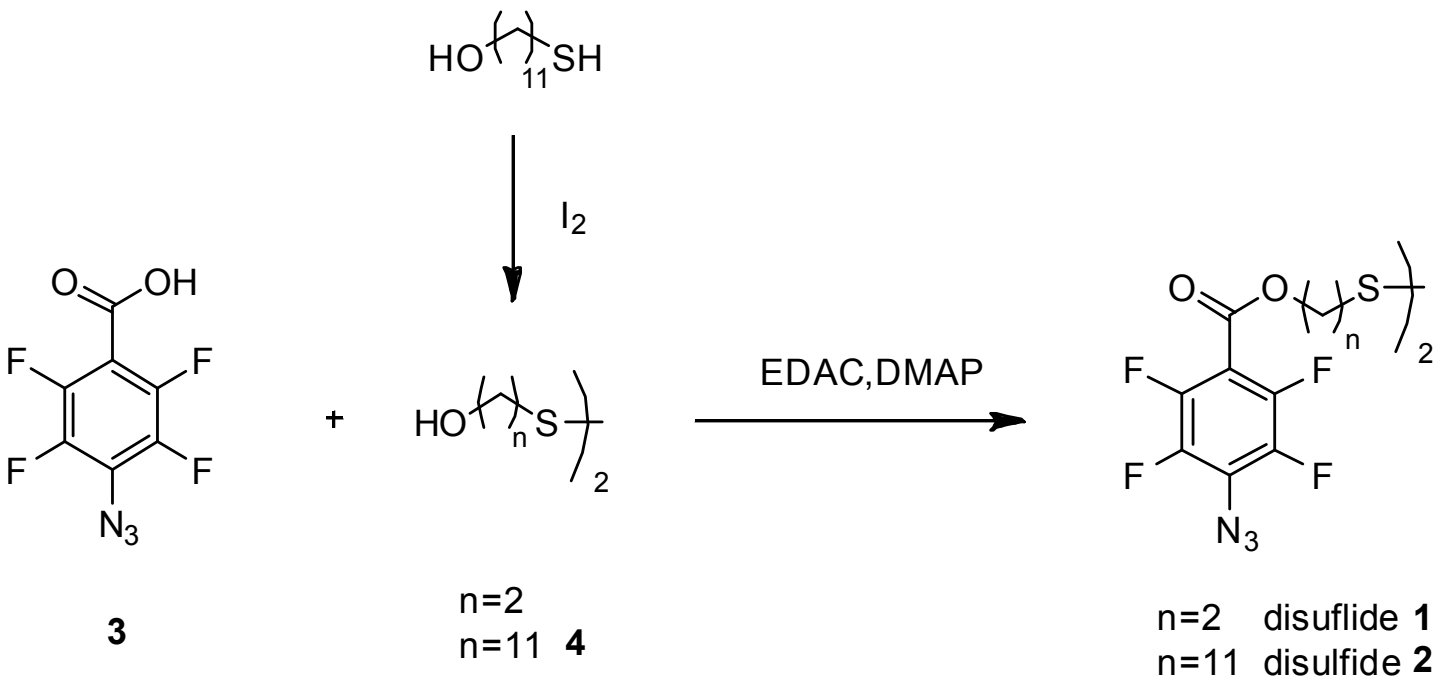

Figure 2.3. Synthesis of PFPA disulfides 1 and 2. 
Synthesis.

PFPA-disufide 1 was prepared following a previously reported procedure. ${ }^{128}$

\section{1,11'-Disulfanediylbi(undecane-11,1-diyl)bis(4-azido-2,3,5,6-tetrafluorobenzoate)}

(PFPA-disufide 2). 11-Mercapto-1-undecanol (90 mg, $0.42 \mathrm{mmol}$ ) in absolute ethanol $(15 \mathrm{~mL})$ was titrated with a saturated solution of iodine in ethanol until the brown color of iodine persisted. The solution was concentrated to $2 \mathrm{~mL}$ and then water $(15$ $\mathrm{mL})$ was added. The solution was extracted using diethyl ether ( $3 \times 15 \mathrm{~mL})$, and the combined ethereal extracts were washed with brine, dried over $\mathrm{NaSO}_{4}$, and the solvent was removed under reduced pressure to afford the disulfide $\mathbf{4}$ as a brown oil. A solution of $3^{129}$ (100 mg, $\left.0.42 \mathrm{mmol}\right)$ in $\mathrm{CH}_{2} \mathrm{Cl}_{2}(15 \mathrm{~mL})$ was cooled to $0{ }^{\circ} \mathrm{C}$, and $N, N^{\prime}$-dimethylaminopyridine (DMAP) $(5.2 \mathrm{mg}, 0.042 \mathrm{mmol})$ and 1-ethyl-3-(3dimethylaminopropyl) carbodiimide hydrochloride (EDAC) (88.6 mg, $0.046 \mathrm{mmol})$ were added. The disulfide 4 was then added and the solution was stirred for $1 \mathrm{~h}$, after which the solution was allowed to warm to room temperature, and stirred for $12 \mathrm{~h}$. The product was recovered by extraction with $\mathrm{CH}_{2} \mathrm{Cl}_{2}$. The organic layer was washed with water, brine, and dried over $\mathrm{Na}_{2} \mathrm{SO}_{4}$. Purification of the crude product was carried out by flash column chromatography with $10 / 1 \mathrm{v} / \mathrm{v}$ hexane/ethyl acetate to afford PFPAdisulfide 2 as clear oil (74.1 mg, 42\%). ${ }^{1} \mathrm{H}$ NMR (400 MHz, $\left.\mathrm{CDCl}_{3}\right): \delta(\mathrm{ppm}) 4.36(\mathrm{t}$, $J=7.4 \mathrm{~Hz}, 2 \mathrm{H}), 2.68(\mathrm{t}, J=7.2 \mathrm{~Hz}, 2 \mathrm{H}), 1.76-1.72(\mathrm{~m}, 4 \mathrm{H}), 1.41-1.28(\mathrm{~m}, 14 \mathrm{H}) .{ }^{13} \mathrm{C}$ NMR (100 MHz, $\left.\mathrm{CDCl}_{3}\right): \delta(\mathrm{ppm}) 159.4,146.0\left(\mathrm{~d}, J_{\mathrm{C}-\mathrm{F}}=258 \mathrm{~Hz}\right), 140.4\left(\mathrm{~d}, J_{\mathrm{C}-\mathrm{F}}=255\right.$ 
Hz), 124.7, 107.1, 63.8, 38.7, 34.4, 31.5, 29.8, 28.8, 28.1, 27.7, 25.1. Anal. Calcd for

$\mathrm{C}_{36} \mathrm{H}_{44} \mathrm{~F}_{8} \mathrm{~N}_{6} \mathrm{O}_{4} \mathrm{~S}_{2}:$ C, 51.42; H, 5.27; N, 9.99. Found: C, 51.50; H, 5.31; N, 10.01.

\section{Sample preparation}

The gold nanoparticles were prepared following a modified procedure of the twophase system. A $0.25 \mathrm{mM}$ aqueous solution $(100 \mathrm{~mL})$ of $\mathrm{HAuCl}_{4}$ (Aldrich) was heated to boiling and $1 \mathrm{wt} \%$ sodium citrate solution $(1.8 \mathrm{~mL})$ was added quickly under vigorous stirring. The solution was allowed to boil for an additional 5 min until the color of the solution became dark purple and finally light red. A $1.7 \mathrm{mM}$ solution of PFPA-disulfide $\mathbf{1}^{128}$ or $\mathbf{2}$ in acetone ( $5 \mathrm{~mL}$ ) was added slowly to the Au NP solution, and the solution was stirred for 10 hours when it turned to burgundy color. Toluene $(15 \mathrm{~mL})$ was subsequently added, and the mixture was vigorously stirred for 1 hour leaving behind a light pink aqueous phase. The toluene layer was then separated, concentrated to $5 \mathrm{~mL}$ using a rotary evaporator at $45{ }^{\circ} \mathrm{C}$, and then diluted with acetone (20 mL). The diluted solution was kept in refrigerator overnight, and centrifuged at 14,000 rpm for $30 \mathrm{~min}$. Precipitates were collected and re-dissolved in acetone by sonication for $1 \mathrm{~min}$, and was further centrifuged. The re-dissolution and centrifugation processes were repeated 3 times to remove the excess PFPA-disulfide. The functionalized Au NPs were kept in acetone for storage. To determine the concentration of functionalized Au NPs, an aliquot of the solution was centrifuged, and the precipitate collected, dried, and weighed. 
Carbohydrates were coupled to Au NPs according to the following general procedure using $\alpha$-1,4-mannobiose as the example. A solution of PFPA-functionalized gold nanoparticles $(1.5 \mathrm{~mL})$ was mixed with $2.9 \mathrm{mM}$ of $\alpha$-1,4-mannobiose aqueous solution $(0.1 \mathrm{~mL}, \mathrm{~V}-\mathrm{Labs})$ in a short flat beaker. The mixture was covered with a $280-\mathrm{nm}$ longpath optical filter (WG-280, Schott Glass) and was irradiated with a 450-W medium pressure $\mathrm{Hg}$ lamp (Hanovia) for 5 min under vigorous stirring. Centrifugation of the solution at 14,000 rpm for 15 min separated the mannobiose-attached gold nanoparticles as precipitates. Excess mannobiose was removed by rinsing the nanoparticles with water 3 times and centrifugation.

\section{Carbohydrate density determination}

A freshly-prepared anthrone solution in concentrated $\mathrm{H}_{2} \mathrm{SO}_{4}(0.5 \mathrm{wt} \%, 1 \mathrm{~mL})$ was added into various concentrations of D-mannose in water $(0.5 \mathrm{~mL})$ in ice bath under stirring. The solution was then heated to $100^{\circ} \mathrm{C}$ and stirred for $10 \mathrm{~min}$. After cooled to room temperature, the UV-vis spectra of the resulting solutions were recorded on a Perkin-Elmer Lamda 45 UV-vis spectrometer. The absorbance of the solution at 620 $\mathrm{nm}$ was measured and the data were plotted against the concentration of D-mannose. The result was used as the calibration curve for the calculation of the ligand density on Au NPs. Ligand density experiments for Au NPs were carried out by dissolving freshly-prepared D-mannose-conjugated Au NPs (0.3-0.5 mg, measured by drying the $\mathrm{Au}$ NPs solution under the reduced pressure) in $0.5 \mathrm{~mL}$ Milli-Q water, and the solutions were treated with anthrone $/ \mathrm{H}_{2} \mathrm{SO}_{4}$ following the same protocol described 
above.The absorbance of Au NPs were deducted from the total singals measured as the bakground, and the density of D-mannose immobilized was then determined using the calibration curve.

\section{Lectin binding assay}

The binding affinity of carbohydrates conjugated on Au NPs was evaluated using Con A according to the following procedure. The binding studies with other lectins (letin from Griffonia simplicifolia (GS II), lectin from Arachis hypogaea (peanut) (PNA), lectin from Glycine max (soybean), Sigma) were carried out in the similar manner. In the experiment, the nanoparticles were incubated in a $10 \mathrm{mM} \mathrm{pH} 7.4 \mathrm{PBS}$ buffer solution containing $0.1 \%$ Tween 20 and 3\% bovine serum albumin (BSA) for $30 \mathrm{~min}$, centrifuged, and incubated in a pH 7.4 PBS solution without BSA for another $20 \mathrm{~min}$. The nanoparticles were subsequently treated with $10 \mu \mathrm{g} / \mathrm{mL}$ Con A (from Canavalia ensiformis (Jack bean), Sigma) solution in $\mathrm{pH} 7.4 \mathrm{PBS}$ buffer $(0.05 \mathrm{~mL})$ containing 10 $\mathrm{mM} \mathrm{MnCl} 2$ and $\mathrm{CaCl}_{2}$ for 1 hour while shaking. In cases where aggregation was induced after binding with Con $\mathrm{A}$, the suspension was transferred to a centrifuge tube and centrifuged at $14,000 \mathrm{rpm}$ for $15 \mathrm{~min}$.

\section{Results and discussion}

PFPA-disulfides $\mathbf{1}$ and $\mathbf{2}$ were synthesized by coupling PFPA-COOH $\mathbf{3}$ with the corresponding diol using EDAC (Figure 2.3). Diol 4 was prepared by oxidizing the hydroxythiol with $\mathrm{I}_{2}{ }^{130}$ The two disulfides were chosen differing in the length of the 
spacer linkage. A one-pot procedure was developed to simultaneously synthesize and functionalize Au NPs with PFPA. Colloidal Au NPs, $\sim 20 \mathrm{~nm}$ in diameter, were prepared using the citrate reduction reaction of $\mathrm{HAuCl}_{4}$. The resulting citratestabilized Au NPs were light red in color exhibiting the surface plasmon absorption peak at $\sim 520 \mathrm{~nm}$ in the UV-vis spectrum. The Au NPs were subsequently functionalized with PFPA-disulfide (Figure 2.3) via a modified phase-transfer ligandexchange reaction, after which the PFPA-functionalized Au NPs migrated to the organic phase, indicating that the hydrophilic surface of citrate-stabilized $\mathrm{Au}$ nanoparticles became hydrophobic. The successful functionalization of Au NPs with PFPA was confirmed by ${ }^{1} \mathrm{H}$ NMR and FTIR (see Figures 2.7 and 2.8 in Supporting Information). The subsequent coupling of carbohydrates to PFPA-functionalized nanoparticles was carried out by mixing an aqueous solution of the carbohydrate with the nanoparticles in acetone, and irradiating the mixture with $>280 \mathrm{~nm}$ light. FTIR spectra of the resulting nanoparticles show that the characteristic $-\mathrm{N}_{3}$ absorption at $2125 \mathrm{~cm}^{-1}$ disappeared (see Figure 2.8), indicating that the azido groups were activated. The mannobiose coated NPs could be redispersed in water without significant change in optical density due to coating of high-density carbohydrate ligands, and excessive unattached carbohydrate was completely removed through water rinses. Accompanied by each step of the surface functionalization is the color change of the nanoparticle solution, shown in Figure 2.4a where $\alpha$-1,4-mannobiose was coupled on Au NPs. The UV-vis spectra of PFPA-Au and mannobiose-Au both underwent red-shifts after 
surface functionalization ( $8 \mathrm{~nm}$ and $4 \mathrm{~nm}$, respectively), likely due to the slight size growth and the change of environment around the nanoparticles.

The amount of carbohydrate ligands coupled to Au NPs was determined by a colorimetry method using anthrone/sulfuric acid. ${ }^{131,132}$ This is a well-established assay for the quantitative analysis of carbohydrates, and has been adopted in glyconanoparticle analysis. ${ }^{133}$ We investigated the ligand density on Au NPs using Dmannose. A calibration curve was obtained by treating various concentrations of Dmannose with anthrone/sulfuric acid, and the absorption at $620 \mathrm{~nm}$ was measured (Figure 2.9 in Supporting Information). Au NPs with D-mannose immobilized were subjected to the same assay and the absorptions at $620 \mathrm{~nm}$ were recorded. The amount of D-mannose attached to Au NPs was subsequently derived from the calibration curve, which averaged at $24 \mathrm{nmol} / \mathrm{mg}$ Au NPs, or 1,200 molecules per Au NP. Assuming that D-mannose ligands are close-packed on the NP, the maximal amount of D-mannose that can occupy on each 20 -nm Au NP was calculated to be $72 \mathrm{nmol} / \mathrm{mg}$ Au NPs, or 3,500 molecules per Au NP (see Supporting Information for detailed calculation). This result corresponds to a surface coverage of $34 \%$ of the photocoupled D-mannose, indicating a fairly reasonable coupling efficiency. 


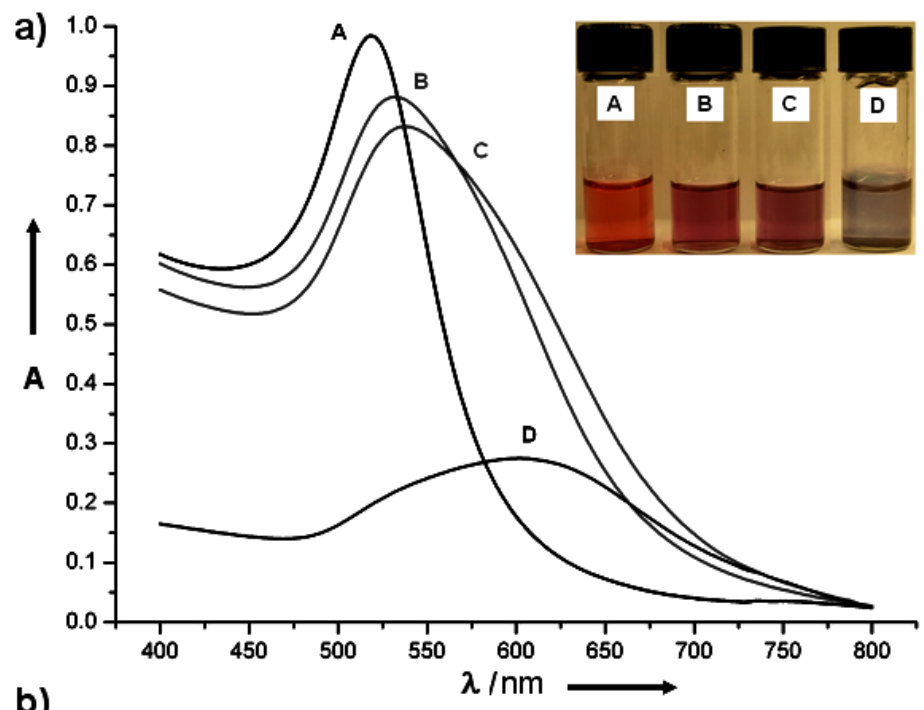

b)
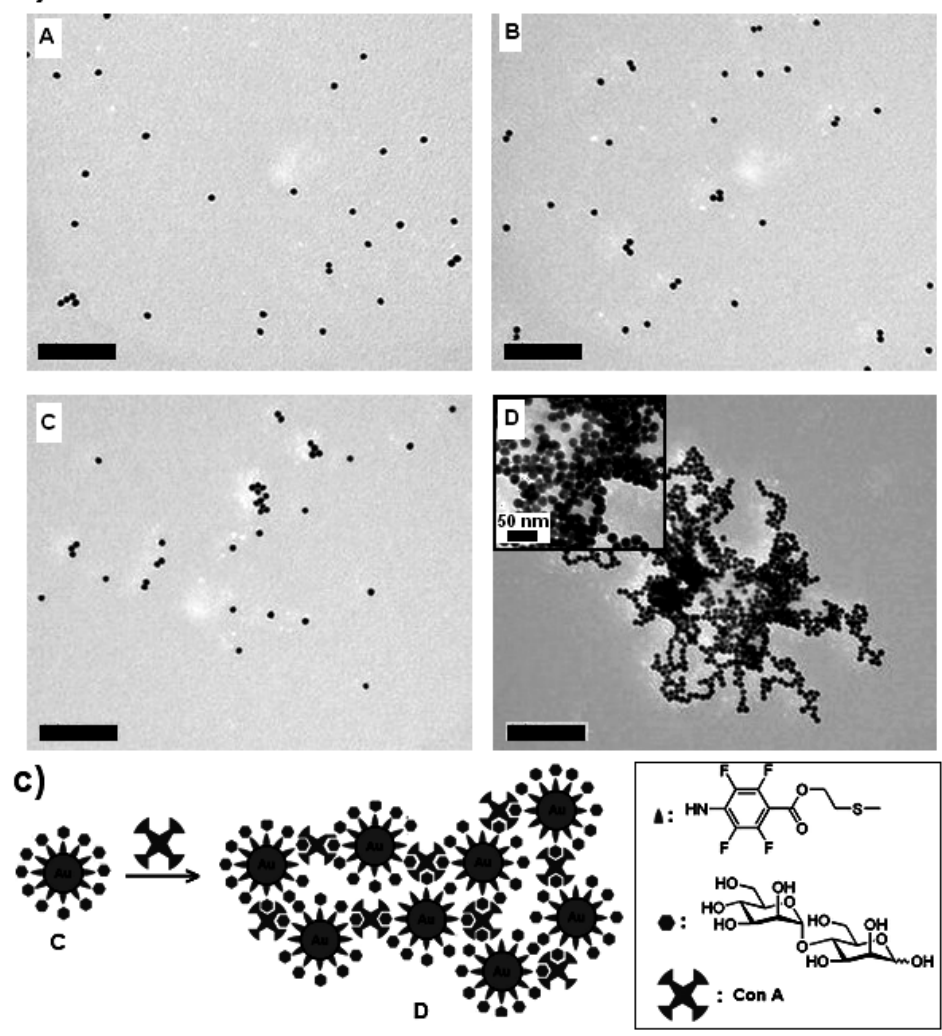

Figure 2.4 (a) UV-vis spectra (insert: Au nanoparticle solutions) b) TEM micrographs (scale bar: $200 \mathrm{~nm}$ ) of Au NPs (A), PFPA-disulfide-functionalized Au NPs (B), Au NPs with surface-coupled $\alpha$-1,4-mannobiose (C), and subsequent treatment with Con A (D). c) Schematic illustration of the interaction of mannobiose-coupled Au NPs with Con $\mathrm{A}$, and the formation of $\mathrm{Au}$ NP aggregates. 
To further investigate the efficiency of the photocoupling reaction, various concentrations of D-mannose were used when mixing with PFPA-functionalized Au NPs during light activation. Here PFPA-disulfide 2 was used in the study. The amount of D-mannose attached to Au NPs was then determined by the anthrone/sulfuric acid assay described above, and results are summarized in Table 2.1. At lower ligand loading, the coupling yield was high but the surface coverage was low. As the amount of added ligand increased, the coupling efficiency decreased whereas the surface coverage increased drastically before saturating at around $80 \%$. Note that even at low ligand loading concentration of $100 \mathrm{nmol} / \mathrm{mg}$ NPs, high surface coverage (80\%) was obtained while relatively high coupling efficiency of $57 \%$ was achieved. The result is significant that large excess of ligand is unnecessary, which is especially beneficial to carbohydrates that are difficult or costly to obtain. Moreover, this approach provides a simple means to control the ligand density on the NPs. Nanoparticles with coupled Dmannose density varying over 3 orders of magnitude can be produced by changing the amount of the ligand initially added. Note that the amount of D-mannose coupled to Au NPs functionalized with PFPA-disulfide $2(57.4 \mathrm{nmol} / \mathrm{mg})$ was higher than that on NPs functionalized with $1(31.6 \mathrm{nmol} / \mathrm{mg})$ with the same initial ligand concentration, demonstrating that the longer spacer increased the coupling efficiency. 
Table 2.1. Coupling efficiency and surface coverage of D-mannose immobilized on Au NPs using PFPA-disulfide 2 as the coupling agent.

\begin{tabular}{|l|l|l|l|}
\hline $\begin{array}{l}\text { Mannose Added } \\
(\mathrm{nmol} / \mathrm{mg} \mathrm{NPs})\end{array}$ & $\begin{array}{l}\text { Mannose Coupled } \\
(\mathrm{nmol} / \mathrm{mg} \text { NPs })\end{array}$ & $\begin{array}{l}\text { Coupling Yield } \\
(\%)[\mathrm{a}]\end{array}$ & $\begin{array}{l}\text { Surface Coverage } \\
(\%)[\mathrm{b}]\end{array}$ \\
\hline 0.1 & 0.081 & 85 & 0.11 \\
\hline 0.5 & 0.40 & 80 & 0.56 \\
\hline 1 & 0.78 & 78 & 1.1 \\
\hline 5 & 3.1 & 61 & 4.3 \\
\hline 10 & 5.3 & 53 & 7.4 \\
\hline 50 & 30 & 59 & 41 \\
\hline 80 & 44 & 56 & 62 \\
\hline 100 & 57 & 57 & 80 \\
\hline 120 & 58 & N/A & 80 \\
\hline 150 & 57 & N/A & 80 \\
\hline
\end{tabular}

[a] Coupling Yield = Mannose Coupled/Mannose Added x 100\%. [b] Surface

Coverage $=$ Mannose Coupled/Max. Mannose Computed x 100\%. The Max. Mannose Computed is $71.7 \mathrm{nmol} / \mathrm{mg}$ NPs for $20-\mathrm{nm}$ Au NPs.

The carbohydrate-functionalized NPs were subsequently subjected to binding studies with a series of lectins, i.e. carbohydrate-binding proteins, to investigate whether the coupled carbohydrates retained their binding affinity. Concanavalin A (Con A), a mannose-binding protein, was used as a model system to test the effectiveness of this coupling chemistry. At $\mathrm{pH}>7$, Con A is tetrameric, each monomer having one 
saccharide binding site specific for mannose, and to a lesser extent, glucose. ${ }^{114}$ Upon treating the mannobiose-Au NPs with Con A, rapid and drastic color change occurred (D, Figure 2.4a insert), and the UV-vis spectrum of the resulting solution showed a large SPR red-shift of $\sim 75 \mathrm{~nm}$ (D, Figure 2.4a). Simultaneously observed was the cluster formation causing broadening of the SPR peak and a decrease in the absorption intensity. The aggregation of the nanoparticles is likely a result of Con A's multiple binding sites for mannose bringing together dimannose-modified nanoparticles. Indeed, TEM micrographs showed that the nanoparticles were discrete and isolated until the addition of Con A (Figure 2.4b). The tetrameric Con A acted as a crosslinking agent that agglomerated mannose strongly, forming larger sizes of nanoclusters (Figure 2.4c). A control experiment was carried out where PFPA-functionalized Au NPs were treated with Con A. No SPR peak shift was observed in the UV-vis spectrum of the resulting solution (Figure 2.11 in Supporting Information). Therefore the SPR shift and agglomeration can only be attributed to the carbohydrate ligands on the Au NPs. 

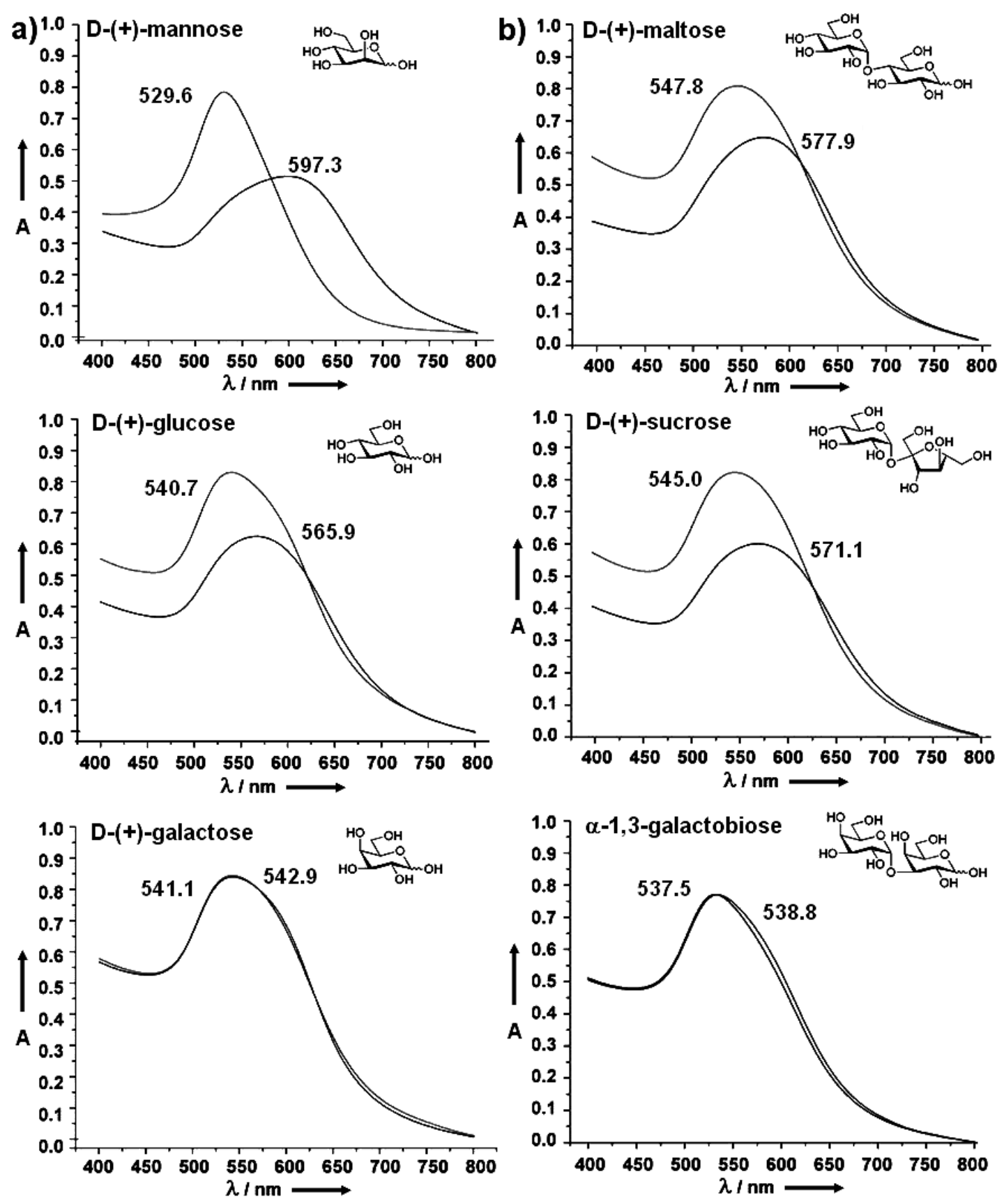

Figure 2.5 UV-vis spectra of (a) monosaccharide-, and (b) disaccharide-functionalized $\mathrm{Au}$ NPs before and after binding with Con A. Only one set of experimental data is shown here. The experiments were, however, repeated over 5 times and the results were consistent and reproducible. 
To further investigate the generality of this coupling chemistry and the specificity of surface-bound carbohydrates, monosaccharides (D-mannose, D-glucose, D-galactose) and disaccharides (maltose, sucrose, $\alpha$-1,3-galactobiose) were coupled to the PFPAfunctionalized Au NPs using the same experimental protocol. The resulting carbohydrate-NPs were subsequently treated with Con A and the UV-vis spectra were recorded. D-Glucose is a known ligand of Con A with a lower binding strength than that of D-mannose, and D-galactose is a non-binding ligand for Con A. ${ }^{134,135}$ The binding constants vary depending on the measurement methods. In the work of Mandal et al., the association constants $\left(\mathrm{K}_{\mathrm{a}}\right)$ of D-glucose and D-mannose were reported to be $1.96 \times 10^{3} \mathrm{M}^{-1}$ and $8.2 \times 10^{3} \mathrm{M}^{-1}$, respectively, measured by isothermal microcalorimetry. ${ }^{136}$ In our studies, the UV-vis spectra of the monosaccharidemodified Au NPs showed the largest SPR red-shift for D-mannose $(67.7 \mathrm{~nm})$ compared to D-glucose $(25.2 \mathrm{~nm})$, whereas almost no change was observed for Dgalactose-functionalized NPs (Figure 2.5a). These results correlated well with the affinity ranking of the free monosaccharides with Con A in solution. ${ }^{136}$ Similar results were also observed for disaccharide-functionalized NPs. Maltose, having two Dglucose units, showed a red-shift of $30.1 \mathrm{~nm}$ whereas sucrose, containing one Dglucose unit, gave a red-shift of $26.1 \mathrm{~nm}$ upon binding with Con A. Almost no change in SPR absorption was observed for galactobiose which is consisted of two nonbinding D-galactose units (Figure 2.5b). 


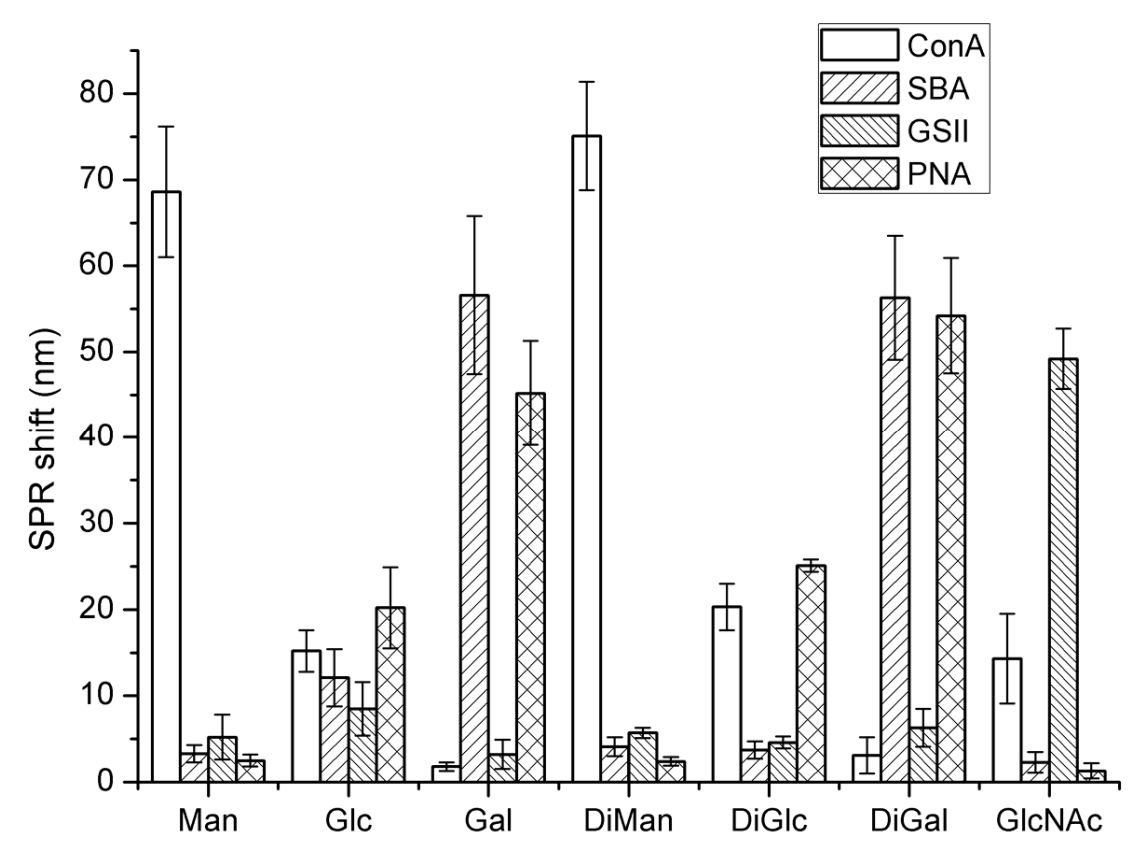

Figure 2.6 SPR peak shifts of carbohydrate-functionalized Au NPs after treating with various lectins. Each data was an average of 5 samples.

Additional cross-reactivity studies were conducted by treating Au NPs conjugated with mono- and di-saccharides (D-mannose (Man), D-glucose (Glc), D-galactose (Gal), $\alpha$-1,4-mannobiose (DiMan), $\beta$-1,3-glucobioses (DiGlc), $\alpha$-1,3-galactobiose (DiGal) and $N$-acetyl-D-glucosamine (GlcNAc)), with Con A and 3 other lectins (GSII, PNA and SBA). The SPR peak shifts were determined from the UV-vis spectra and are shown in Figure 2.6. The affinity ranking derived from the SPR peak shift directly correlates with reported solution binding affinity between each carbohydrate and lectin. ${ }^{68,114}$ For example, larger shifts were observed for the carbohydrate-lectin pairs of Gal-SBA, Gal-PNA, DiGal-SBA, DiGal-PNA, and GlcNAc-GSII, which was consistent with our previous study using a carbohydrate microarray where strong interactions were also observed for these carbohydrate-lectin pairs. ${ }^{128}$ 


\section{Conclusions}

In summary, we have developed a general method for coupling carbohydrates to gold nanoparticles. The method is based on the photochemically induced $\mathrm{CH}$ insertion reactions of PFPAs, and it does not require chemical derivatization of the carbohydrate structures. Furthermore, the coupling reaction is fast, taking place in minutes instead of hours which is needed in most thermally-initiated conjugation reactions. The coupling efficiencies were high, and surface coverage of over $80 \%$ was obtained. The coupled carbohydrates effectively retained their recognition abilities as demonstrated by the strong interactions with their corresponding carbohydrate-binding proteins. In addition, the binding affinities of surface-bound carbohydrates with various lectins were consistent with those of the free carbohydrates with the corresponding lectins in solution. The sensitive SPR signal was conveniently used to monitor the surface chemistry occurred on the nanoparticles, especially in examining the interactions of surface-bound carbohydrates with their binding proteins where large SPR red-shifts were observed causing visible color changes of the Au NP solutions. The method developed can be readily applied to other carbohydrate structures, and we have successfully coupled oligosaccharides and polysaccharides using the same approach. This general coupling chemistry together with the convenient optical detection offers an attractive platform for label-free, rapid, and sensitive detection of carbohydratebased molecular recognition. 


\section{Supporting Information}

Characterization of PFPA-functionalized and mannobiose-conjugated Au NPS

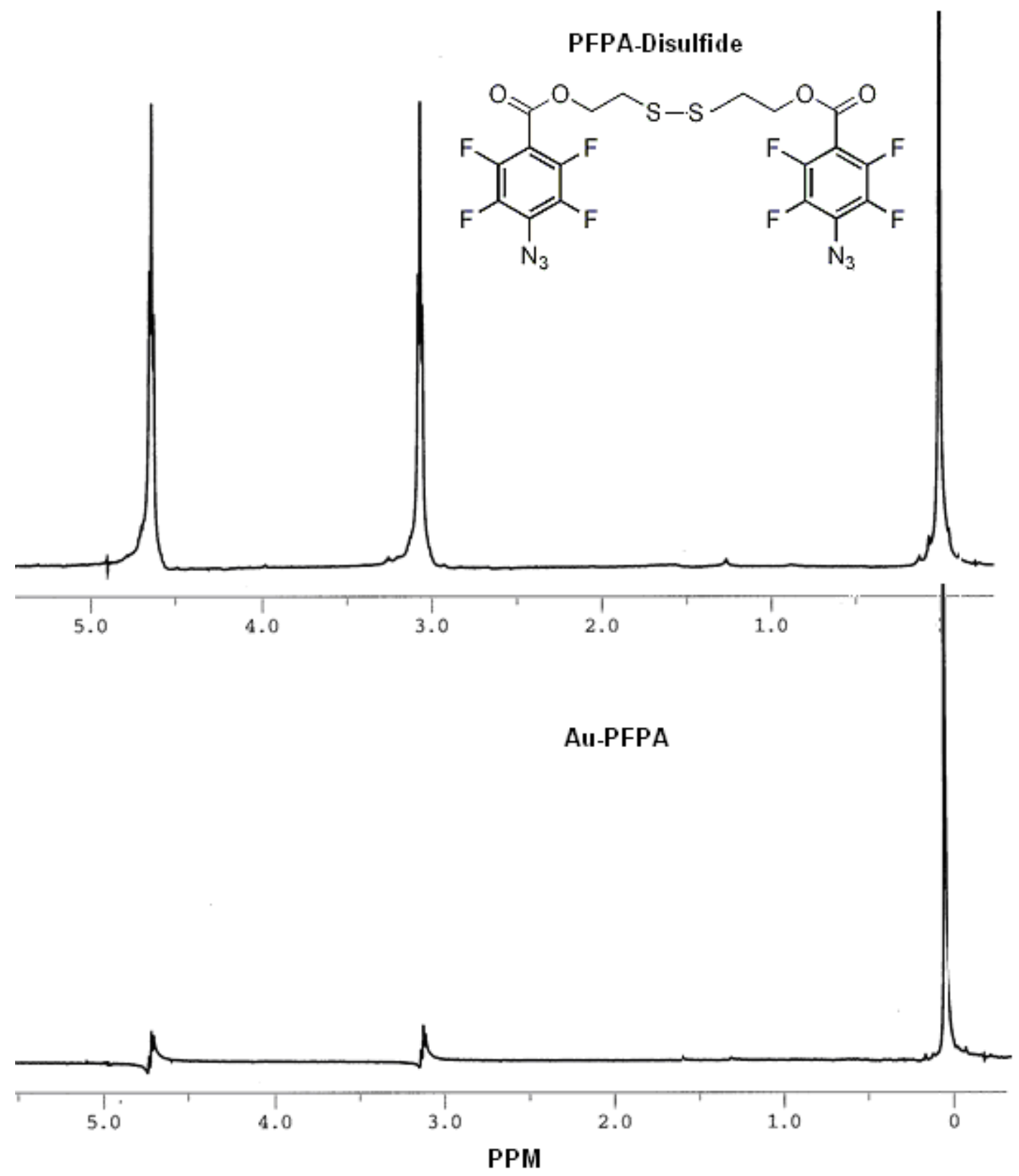

Figure $2.7{ }^{1} \mathrm{H}$ NMR spectra of PFPA-disulfide $\mathbf{1}$ and gold nanoparticles functionalized with PFPA-disulfide 1 (Au-PFPA) in $\mathrm{CDCl}_{3}$ with TMS ( $\left.\delta 0.0 \mathrm{ppm}\right)$. 

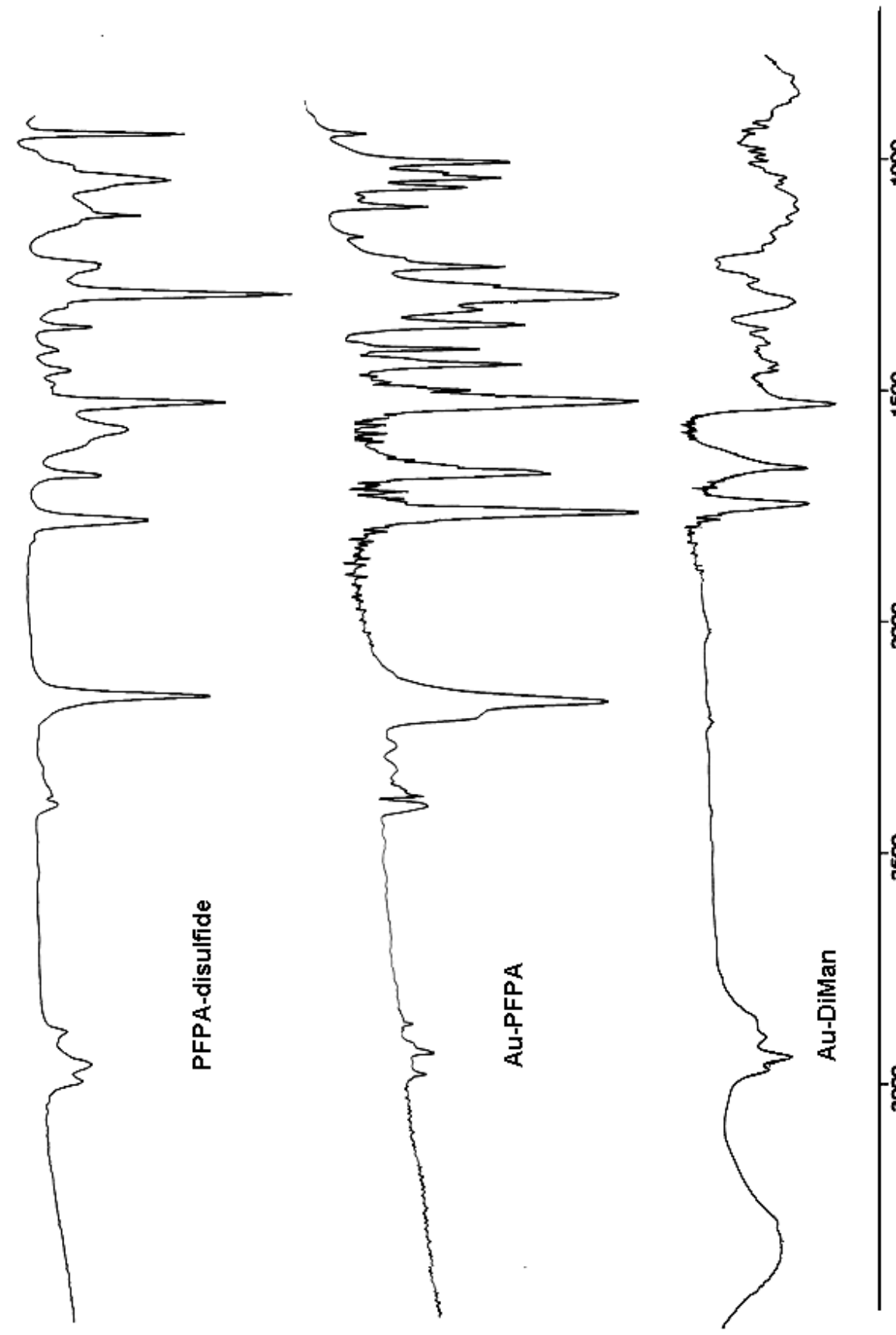

8
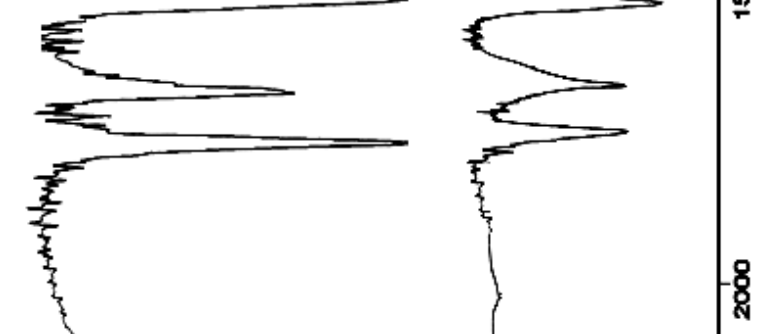

'텅

Figure 2.8 FT-IR spectra of PFPA-disulfide 1, gold nanoparticles functionalized with PFPA-disulfide 1 (Au-PFPA), and Au NPs subsequently coupled with $\alpha-1,4-$ mannobiose (Au-DiMan). The azide $\left(-\mathrm{N}_{3}\right)$ absorption at $\sim 2125 \mathrm{~cm}^{-1}$ disappeared after light activation. 
Mannose density measurement

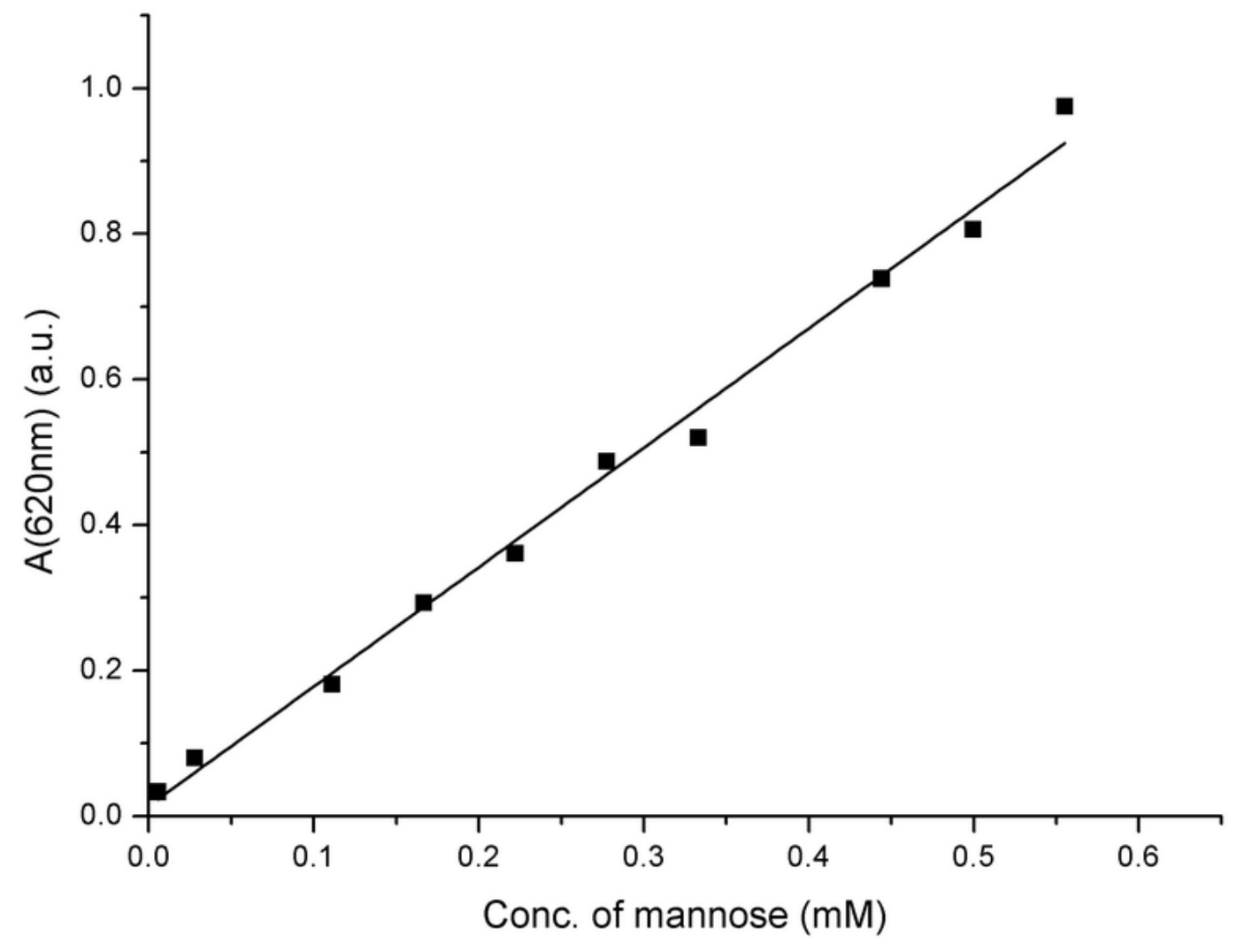

Figure 2.9 Calibration curve obtained by treating various concentrations of Dmannose with anthrone/sulfuric acid and measuring the absorption at $620 \mathrm{~nm}$. 

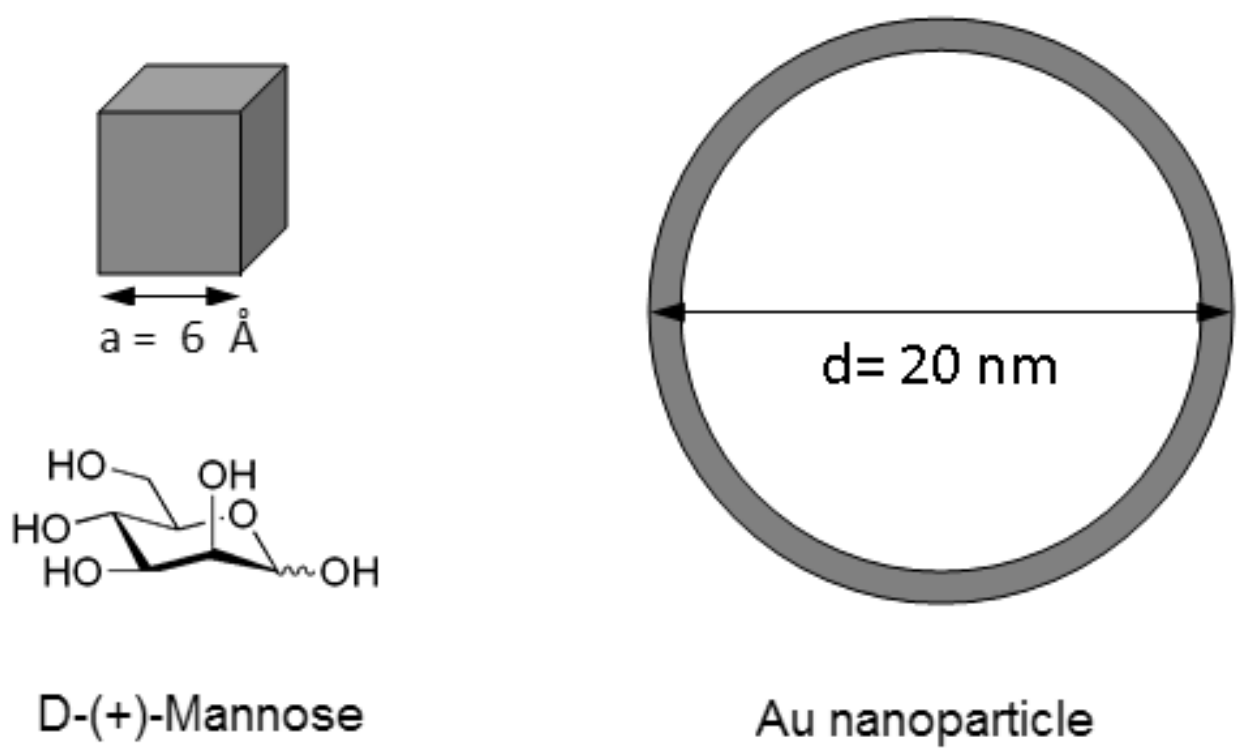

Au nanoparticle

Figure 2.10 D- mannose molecules and Au NPs

The maximal number of D-mannose molecules on each Au NP is calculated as follows.

Assuming that D-mannose occupies in space by taking the shape of a square, each side

of the square is measured to be $\sim 6 \AA$ by Chem $3 \mathrm{D}$ (CambridgeSoft., Ultra, version 9.0).

The D-mannose molecule is then projected to the surface, and the surface area of each

D-mannose molecule is $36 \AA^{2}$.

The surface area of one $20-\mathrm{nm}$ Au NP is $1.26 \times 10^{5} \AA^{2}\left(=4 \pi \times 100^{2}\right)$. 
The maximal number of D-mannose molecules occupying the surface of one Au NP in a closely packed manner is $3,491\left(=1.26 \times 10^{5} \AA^{2} / 36 \AA^{2}\right)$.

The volume of each Au NP is $4.19 \times 10^{6} \AA^{2}\left(=4 / 3 \pi \times 100^{3}\right)$.

Assuming that the density of Au NPs equals to that of gold (19.32 $\mathrm{g} / \mathrm{mol})$, the weight of each $\mathrm{Au} \mathrm{NP}$ is $8.09 \times 10^{-14} \mathrm{mg}$.

$1 \mathrm{mg}$ of Au NPs is equivalent to $0.0205 \mathrm{nmol}\left(1 / 8.09 \times 10^{-14} \times 6.02 \times 10^{23}\right)$.

The moles of D-mannose on $1 \mathrm{mg}$ Au NP is $71.7 \mathrm{nmol}(0.0205 \times 3,491)$.

The D-mannose density of Au NP coated was measured as $24.1 \pm 1.7 \mathrm{nmol} / \mathrm{mg} \mathrm{Au}$ NPs, and the number of D-mannose molecules is $\sim 1,200(=24.1 / 0.0205)$. And the estimated surface coverage is $\sim 34 \%(=1200 / 3491)$ 
Control experiments

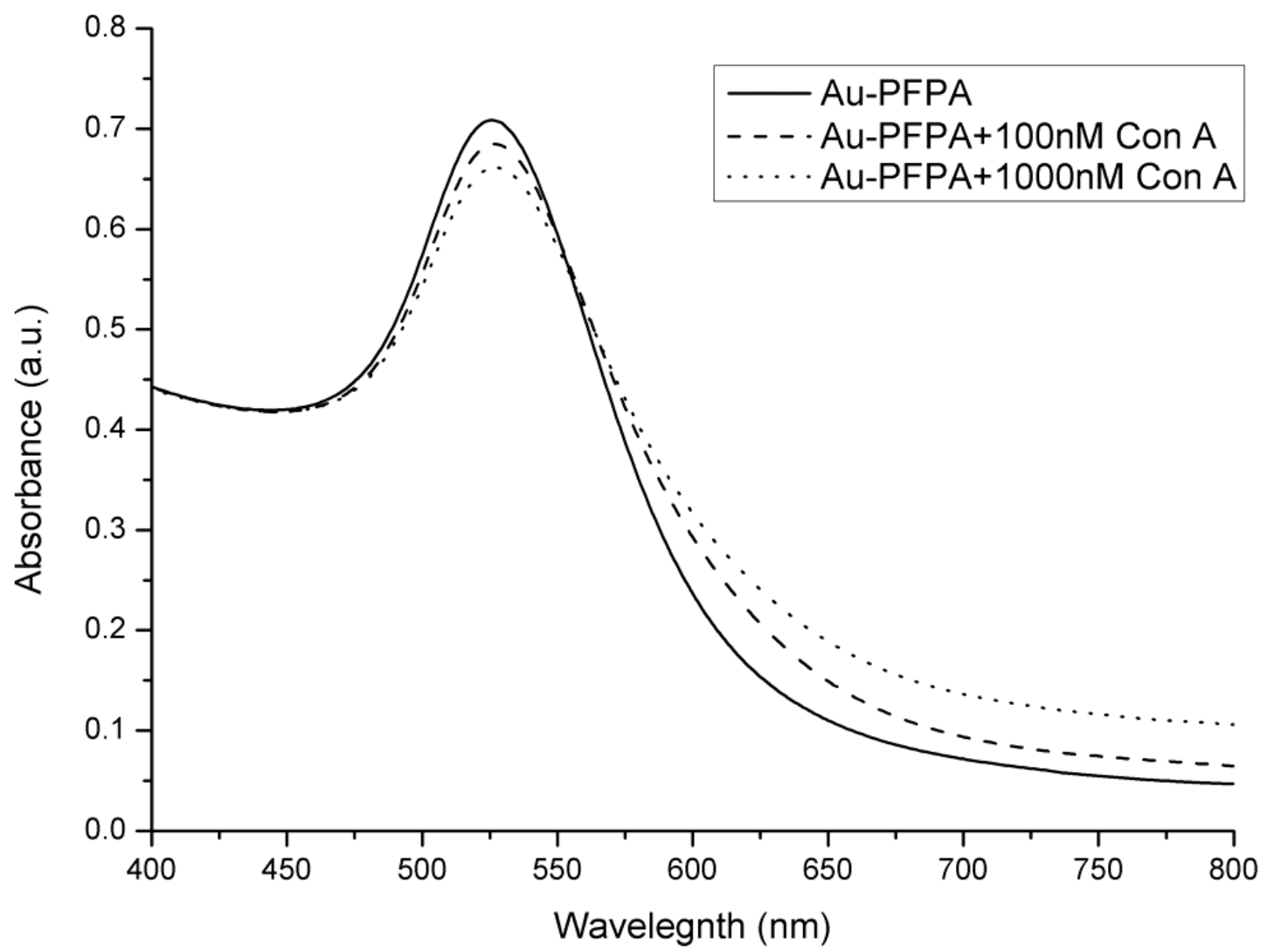

Figure 2.11 UV-vis spectra of PFPA-functionalized Au NPs and after treating with $100 \mathrm{nM}$ and $1000 \mathrm{nM}$ Con A. 


\section{Chapter 3. Quantitative Analysis of Binding Affinity of Glyconanoparticles with Lectins}

The work in Section 3.1 was published on "Wang, X.; Ramström, O.; Yan, M.,

Glyconanomaterials: Synthesis, Characterization, and Ligand Presentation. Adv. Mater. 2010, 22, 1946-1953"

http://onlinelibrary.wiley.com/doi/10.1002/adma.200903908/abstract Reproduced by permission of John Wiley and Sons

The work in Section 3.2 was published on "Wang, X.; Ramström, O.; Yan, M., Dynamic Light Scattering as Efficient Tool to Study Glyconanoparticle-Lectin Interactions. Analyst 2011, 136, 4174-4178"

http://pubs.rsc.org/en/Content/ArticleLanding/2011/AN/c1an15469a Reproduced by permission of The Royal Society of Chemistry

The work in Section 3.3 was submitted for publication. 
Glyconanomaterials are synthesized under specific conditions using different chemistry and reagents. Careful evaluation of these materials must therefore be conducted to fully characterize the structure, composition, density of surface ligands as well as biological activities in order to make proper correlation with their performances. Thanks to the significantly increased specific surface areas of nanomaterials, conventional chemical analytic techniques that are insensitive to flat substrates can be readily adopted for nanomaterial characterization. In Brust's first paper on the preparation of thiol-capped Au NPs, the products were characterized by FTIR showing the presence of alkanethiol, and TEM revealing the size and shape of the nanoparticles. ${ }^{89}$ With the rapid development of advanced analytical tools, especially sensitive surface characterization techniques, nanomaterials can now be analyzed more accurately, providing in-depth understanding of the chemical and physical properties of glyconanomaterials. ${ }^{137}$ NMR, surface-enhanced Raman spectroscopy (SERS), and FTIR offer detailed structural analysis of nanomaterials and surface ligands. Thermogravimetric analysis (TGA) yields the amount of organic components on the nanomaterials, from which the ligand densities can be derived. Elemental analysis and XPS provide information on the elemental composition and chemical state of the bulk nanomaterials and the surface ligands. A combination of microscopy techniques of scanning probe (STM, AFM), TEM and small-angle X-ray scattering (SAXS) reveals the physical characteristics of size, shape, and assembly behaviors of the nanomaterials. ${ }^{138,139}$ Caution should be used when analyzing the results as the experimental conditions applied to each technique (vacuum, ambient, 
solution) can significantly impact the outcome. Microscopic techniques can also be used to directly visualize the interactions of glyconanomaterials with their binding partners. In our study, when D-mannose-functionalized iron oxide nanoparticles were treated with E. coli strain ORN178, the nanoparticles selectively bound to the FimH lectin on the bacteria, which was clearly shown in TEM. ${ }^{140}$ The surfaces can furthermore be characterized by taking advantage of the unique properties offered by the nanomaterials. A classic example is metal nanoparticles, which exhibit SPR that is highly sensitive to the surface constituents and can be conveniently monitored colorimetrically as the molecular recognition event occurs at, or close to, the surface of the nanoparticles. ${ }^{110}$

Biomedical imaging, therapeutics, medical diagnosis, and drug delivery are among the many areas glyconanomaterials have the potential to impact. The interaction of glyconanomaterials with biological receptors and targets is a critical process involved in these applications and the binding affinity is thus an important parameter for evaluating the performance of glyconanomaterials. When a ligand is conjugated to a solid surface, the structure of the ligand is in a sense altered. The binding affinity of the free ligand in solution can no longer be used as the substitute for the glyconanomaterial with the corresponding binding partner. In glyconanomaterials, multiple ligands are clustered on a single solid entity. Multivalency effect comes in play whereby ligands act cooperatively enhancing the overall binding affinity with the 
receptor. This multivalency effect is highly sensitive to how the ligands are presented on the nanomaterial surface, i.e., the number of ligands or ligand density, the structure and length of the spacer linker, and how the ligand is attached or the coupling chemistry. Therefore the binding affinity of the glyconanomaterials must be carefully evaluated taking into consideration these parameters.

Carbohydrate-lectin interactions of free ligands in solution have been studied by many biochemical and biophysical methods including NMR spectroscopy, ${ }^{141}$ SPR spectroscopy, ${ }^{142,143} \mathrm{X}$-ray crystallography, ${ }^{144,145}$ titration microcalorimetry, ${ }^{146}$ and fluorescence spectroscopy. ${ }^{147}$ Quantitative analysis of glyconanomaterials is investigated to a lesser extent, and a few protocols were reported to determine the binding affinity of glyconanoparticles. Lin and coworkers used SPR to analyze the multivalent interactions between mannose, glucose, or galactose-encapsulated gold nanoparticles with Con A. ${ }^{11}$ A competition binding study was carried out where equilibria were established between mannopyranoside attached on the SPR sensor, Con A, and varied concentrations of mannose-encapsulated Au NPs. The dissociation constant $\mathrm{K}_{\mathrm{d}}$ of mannose-Au NPs with Con A was determined to be $2.3 \mathrm{nM}$, representing a binding affinity over 5 orders of magnitude higher than that of the free D-mannopyranoside with Con $A$ in solution $\left(\mathrm{K}_{\mathrm{d}}: 470 \mu \mathrm{M}\right.$ measured by ITC $\left.^{148}\right)$. In the system developed by $\mathrm{Wu}$ et al., magnetite-gold core/shell nanoparticles coated with proteins were allowed to interact with carbohydrate ligands on a glycan array. ${ }^{149} \mathrm{~A}$ magnetic field was applied to amplify the protein-carbohydrate interactions and the 
signals were visualized and quantified using a silver enhancement reagent. Apparent $\mathrm{K}_{\mathrm{d}}$ values of $66 \mathrm{nM}, 61 \mathrm{nM}$ and $57 \mathrm{nM}$ were determined for Man1, Man4 and Man9 ligands with Con A, respectively.

\subsection{Fluorescence-based Competition Assays}

In this part, a fluorescence-based competition assay has been developed to determine the binding affinity of glyconanoparticles to lectins. In the assay, a fixed concentration of a free ligand (for example, D-mannose) and varying amounts of ligands bound to Au NPs were incubated with fluorescein isothiocyanate (FITC)-labeled Con A. The solution was then centrifuged and the fluorescence intensity of the supernatant was measured. Two equilibria co-exist in the system: FITC-Con A with free D-mannose and FITC-Con A with D-mannose bound on nanoparticles (Figure 3.1). Since very low concentrations of Con A and free D-mannose were used, it was assumed that no agglomeration occurred. Both interactions are reversible, and steady equilibria are reached rapidly. 

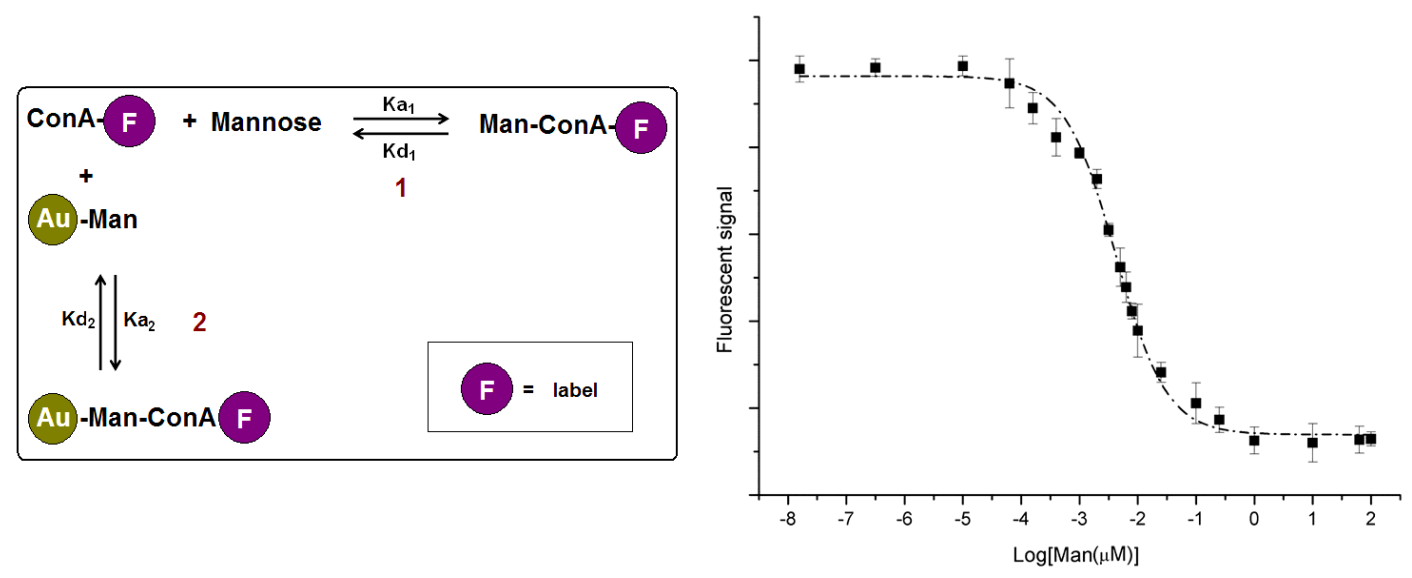

Figure 3.1. Equilibria involved in the competition binding assay (left). Concentration dependent fluorescence intensity curve (right). [Man] is the concentration of Dmannose on NPs determined using the anthrone $/ \mathrm{H}_{2} \mathrm{SO}_{4}$ colorimetry assay described in the text. Each data point was an average of 3 assays.

In order to obtain the dissociation constant $\mathrm{K}_{\mathrm{d}}$, the concentration of the carbohydrate ligand on Au NPs must be determined. The colorimetric assay of anthrone-sulfuric acid was adopted to measure the ligand density on the nanoparticles. ${ }^{133}$ A calibration curve was first established using the corresponding free carbohydrate, and the amount of surface-bound ligand on the Au NPs was subsequently determined. The fluorescence intensity measured from the competition studies was plotted against the concentration of bound D-mannose on the Au NPs (Figure 3.1). The resulting curve fits a typical competition assay for ligand-receptor binding, validating the assumptions made for the system. The $\mathrm{IC}_{50}$ value was subsequently derived and the apparent dissociation constant $\left(\mathrm{K}_{\mathrm{d}}\right)$ calculated using the Cheng-Prusoff equation (eq. 3.1), 


$$
K_{d 2}=\frac{I C_{50}}{1+\frac{[M]}{K_{d 1}}}
$$

where $\mathrm{IC}_{50}=$ concentration of ligands displaying $50 \%$ of specific binding; $[\mathrm{M}]=$ concentration of free ligand, i.e. D-mannose; $\mathrm{K}_{\mathrm{d} 1}=$ dissociation constant of free ligand to Con $\mathrm{A}$; and $\mathrm{K}_{\mathrm{d} 2}=$ dissociation constant of surface bound D-mannose to Con A. 


\subsection{Dynamic Light Scattering}

A number of analytical techniques have been used to monitor the interactions of GNPs with biological receptors, including UV-vis spectroscopy, ${ }^{150}$ transmission electron microscope (TEM), ${ }^{151}$ surface plasmon resonance (SPR), quartz crystal microbalance $(\mathrm{QCM}),{ }^{152}$ isothermal titration calorimetery (ITC), ${ }^{94}$ magnetic resonance imaging (MRI), ${ }^{153}$ and fluorescence spectroscopy. ${ }^{154,155}$ Each technique has its advantages and limitations, and some methods are restricted to the property of the nanomaterials. For instance, UV-vis spectroscopy only applies to metal nanoparticles that absorb light by free electron oscillations. SPR and QCM are generally performed on Au surfaces where the interactions of nanoparticles with target biomolecules immobilized on $\mathrm{Au}$ surfaces are monitored.

Light scattering is a powerful technique for characterizing particles in solutions. When a beam of light passes through a colloidal dispersion, the particles scatter the light in all directions. In DLS, the particles are illuminated with a monochromatic laser. The intensity of the scattered light fluctuates and the rate is dependent on the size of the particles. Analysis of the time dependence of the intensity fluctuations yields the diffusion coefficient of the particles, from which the hydrodynamic radius, or the diameter, of the particles can be calculated. ${ }^{156}$ DLS has become a routine analytical tool for particle size measurement. The technique has also been used to study the interactions of nanoparticles with other species such as polymers, ${ }^{157} \mathrm{DNA}^{158-160}$ and biomarkers. ${ }^{161}$ The advantages of DLS are: (1) excellent sensitivity, (2) low-cost, (3) 
easy sample preparation, and (4) fast measurement (data can be obtained in a few minutes). In this part, we report that DLS is a highly efficient technique to study GNP - lectin interactions. GNPs of different sizes and particle composition were synthesized and their interactions with lectins were monitored by DLS. The apparent association constant $\left(\mathrm{K}_{\mathrm{d}}\right)$ values were determined from the particle size concentration response curves. The impact of the particle size on the affinity of the GNPs was also investigated. To the best of our knowledge, this is the first report on the quantitative analysis of GNP - lectin interactions by DLS.

\section{Experimental details}

Preparation of silica NPS

Silica NPs were synthesized following a modified Stöber protocol, ${ }^{162}$ similar to what was previously described. ${ }^{125}$ TEOS $(2 \mathrm{~mL})$ was added to 200 proof absolute ethanol (34 mL) followed by $\mathrm{NH}_{4} \mathrm{OH}(35 \%, 1 \mathrm{~mL})$. The reaction was allowed to proceed at room temperature overnight with vigorous stirring to yield a white colloidal solution; the particle size was $35 \mathrm{~nm}$ measured by DLS. NPs of $110 \mathrm{~nm}$ size was synthesized following the same procedure except for the amount of reagents added: TEOS (2.8 $\mathrm{mL}), \mathrm{NH}_{4} \mathrm{OH}(2.8 \mathrm{~mL})$. The 470-nm NPs were prepared using the seed-growth method. ${ }^{163}$ TEOS $(1.4 \mathrm{~mL})$ was added to 200 -proof absolute ethanol $(34 \mathrm{~mL})$ followed by the addition of $\mathrm{NH}_{4} \mathrm{OH}(35 \%, 2.8 \mathrm{~mL})$. The reaction was allowed to proceed at 
room temperature overnight with vigorous stirring, after which, additional TEOS (1.4 $\mathrm{mL}$ ) was added continuously in aliquots of $0.2 \mathrm{~mL}$ every $10 \mathrm{~min}$.

Functionalization of silica NPS with perfluorophenyl azide (PFPA)

PFPA-silane $(80 \mathrm{mg})$, synthesized following a previously reported procedure, was added directly to the Stöber solution prepared above, and the mixture was stirred at room temperature overnight. The mixture was then brought to reflux under continuous stirring for $1 \mathrm{~h}$ at $\sim 78{ }^{\circ} \mathrm{C}$ to facilitate the silanization of the silica nanoparticles with PFPA-silane. ${ }^{125}$ The mixture was centrifuged at $12,000 \mathrm{rpm}$ for $30 \mathrm{~min}$, and the precipitate was redispersed in the fresh solvent by sonication. This centrifugation/redispersion procedure was repeated three times with ethanol and twice with acetone.

\section{Conjugation of carbohydrates onto PFPA-functionalized NPS}

Our previously reported procedure of coupling carbohydrates onto Au NPs was followed. Briefly, the dispersion of PFPA-functionalized NPs in acetone and an aqueous solution of carbohydrate was placed in a flat-bottom dish, and the mixture was irradiated with a 450-W medium pressure $\mathrm{Hg}$ lamp with a $280-\mathrm{nm}$ filter for 10 min. Excess carbohydrate was removed by membrane dialysis in water for 24 hours. The concentration of the resulting GNPs, ca. $18.4 \mathrm{mg} / \mathrm{mL}$, was determined gravimetrically after drying the solution under reduced pressure for 3 hours. 
Synthesis of 40-nm Au GNPs

The Turkevich method ${ }^{164}$ was followed to synthesize Au NPs. An aqueous solution of sodium citrate $(1 \mathrm{wt} \%, 1.2 \mathrm{~mL})$ was added to a boiling $\mathrm{HAuCl}_{4}$ solution $(0.25 \mathrm{mM}$, $100 \mathrm{~mL}$ ) under vigorous stirring for $15 \mathrm{~min}$. PFPA-disulfide (Figure 3.2), was synthesized following a previously reported procedure. A solution of PFPA-disulfide (25 mg) in acetone $(5 \mathrm{~mL})$ was added to the Au NPs solution, and the solution was stirred for 12 hours. The resulting NPs were centrifuged at 12,000 rpm for $15 \mathrm{~min}$ and cleaned with acetone 3 times. Carbohydrate conjugation followed the same procedure described above for the silica NPs.

\section{Interaction of GNPs with lectins and DLS measurements}

The following general procedure was followed for all GNPs and lectins. GNPs $(0.1$ $\mathrm{mg}$ ) were incubated in a pH 7.2 HEPES buffer $(10 \mathrm{mM}, 0.5 \mathrm{~mL})$ containing $3 \%$ BSA for $30 \mathrm{~min}$. The sample was then centrifuged and the particles rinsed 3 times with the fresh HEPES buffer. The GNPs were subsequently treated with a solution of Con A (or $\mathrm{RCA}_{120}$ ) in $\mathrm{pH} 7.2$ HEPES buffer at different concentrations for 2 hour while shaking. For DLS measurements, the suspension was diluted to $3 \mathrm{~mL}$ using the HEPES buffer. Each DLS measurement was performed at 20 scans, and was repeated 6 times. 


\section{Results and discussion}

\section{Lectin detection using silica GNPs by DLS}

GNPs were synthesized using the methods developed previously in our laboratory. ${ }^{165,166}$ Briefly, silica NPs and Au NPs of varying sizes, synthesized by the Stöber method ${ }^{162}$ and the Turkevich method, ${ }^{164}$ respectively, were functionalized with PFPA-silane or PFPA-disulfide (Figure 3.2a). Man and Gal were then coupled to the NPs by the $\mathrm{CH}$ insertion reaction of PFPA by UV activation. ${ }^{106} \mathrm{Con} \mathrm{A}$, a well-studied lectin that exhibits high affinity for the terminal $\alpha$-D-mannopyranosyl group, ${ }^{114,167}$ was used to evaluate the DLS technique in studying GNP-lectin interactions. As demonstrated in our previous studies, Man-functionalized NPs formed aggregates upon binding with Con $\mathrm{A},{ }^{166}$ resulting in an overall size increase as observed by TEM. $^{155}$ 
(a)

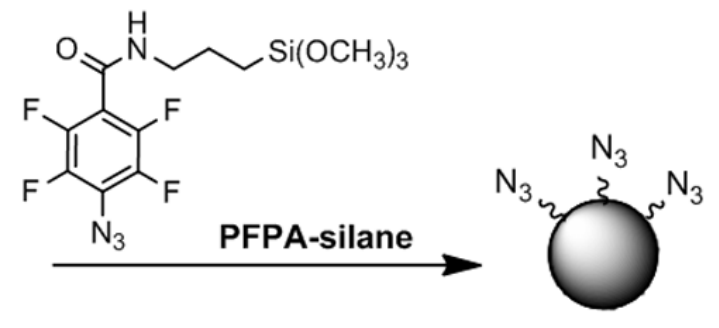

Silica NPs

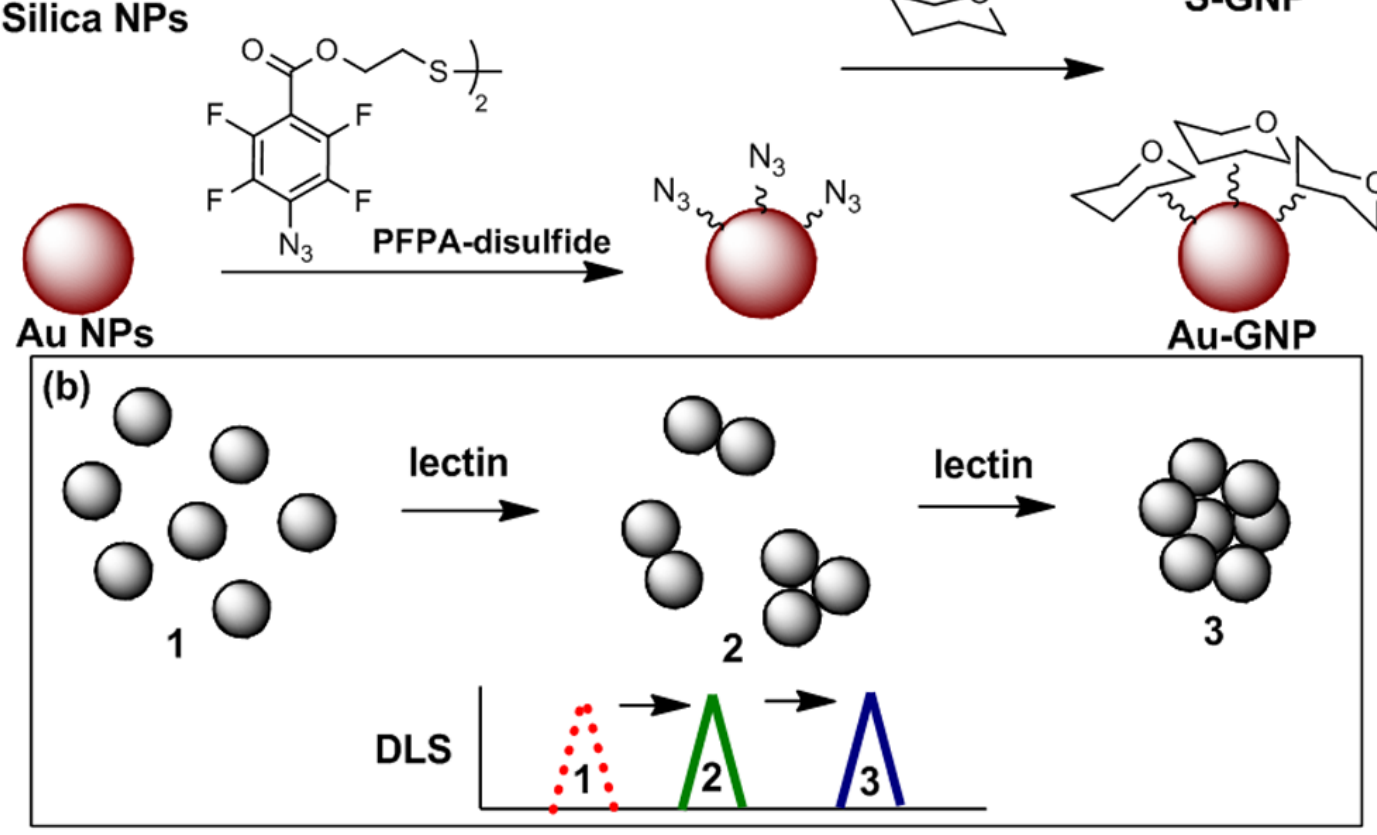

Figure 3.2 (a) Synthesis of silica and Au GNPs: S-GNP and Au-GNP. (b) GNP aggregation upon addition of lectin.

To investigate the feasibility of DLS in studying GNP-lectin interactions, Man coupled on silica NPs $(35 \pm 4.4 \mathrm{~nm}$ in diameter), S-M-GNP, was treated with varying concentration of Con A ( $1 \mathrm{nM}-700 \mathrm{nM})$. The hydrodynamic radius of the resulting complex was measured by DLS. At $1 \mathrm{nM}$ Con A concentration, there was no obvious increase in the average particle size (Figure 3.3a). When a higher concentration of Con A was added (50,70, and $170 \mathrm{nM}$, respectively), the median diameter of the resulting particles increased to $55.1 \pm 4.5,64.3 \pm 9.5 \mathrm{~nm}$, and $94.8 \pm 14.6 \mathrm{~nm}$, respectively (Figure 
3.3b-d). Also increased is the error margin suggesting that particles become more polydisperse with the addition of Con A.
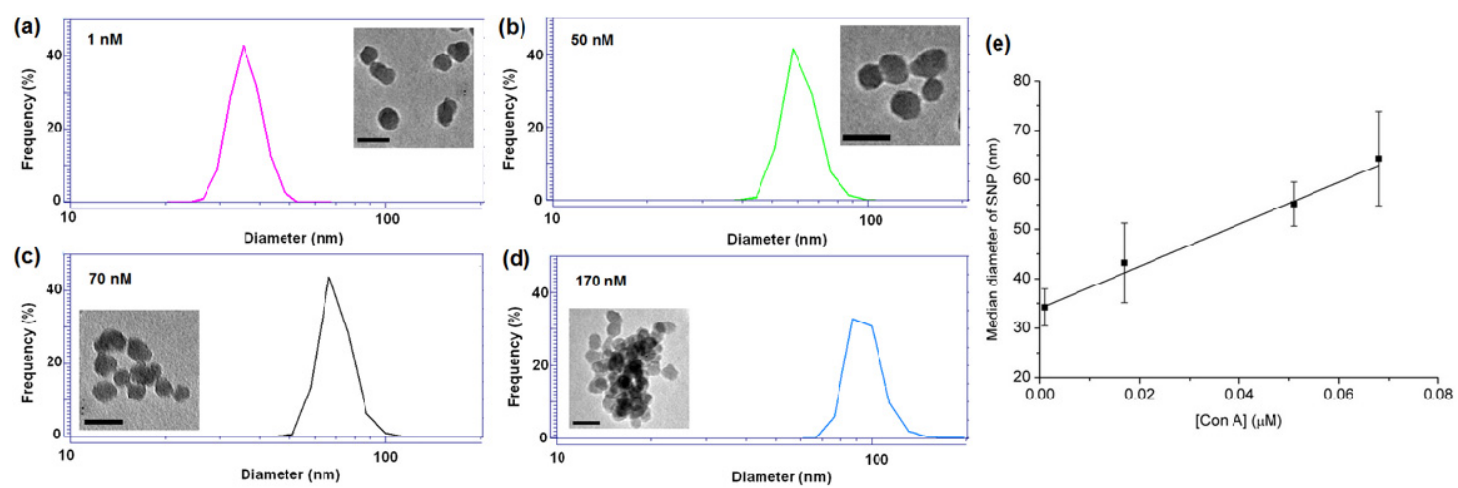

Figure 3.3 (a-d) DLS spectra and TEM images (inserts, scale bars: $50 \mathrm{~nm}$ ) when 35nm S-M-GNP was treated with varying concentrations of Con A. (e) The particle size vs. concentration of Con A.

The sensitivity of the DLS detection was next studied. The limit of detection (LOD), defined as the lowest analyte concentration measurable against the background, was determined from Equation 3.2,

$$
L O D=\frac{3 \sigma}{b}
$$

where $\sigma$ is the standard deviation of the spectroscopic signals of the blank sample (from 12 measurements), and b is the slope of the linear calibration curve. ${ }^{168,169}$ Using the data from Figure 3.3e, the LOD of Con A by the 35-nm S-M-GNP was calculated to be $2.9 \mathrm{nM}$. 
To investigate the generality of this method with respect to the nature of the particles, $\mathrm{Au}$ NPs (40 nm in diameter) were synthesized, and Man was then coupled onto the particles. ${ }^{155}$ The resulting Au-M-GNP was treated with varying concentrations of Con A and the particle sizes were monitored by DLS, from which a calibration curve was established. The LOD was then calculated according to Eq. 1, which yielded $15 \mathrm{nM}$. This value was higher than that of the $35-\mathrm{nm}$ S-M-GNP determined by the same method $(2.9 \mathrm{nM})$, indicating that the Au GNPs were of approximately 5 times lower sensitivity than the silica GNPs in detecting Con A. However, the method was more sensitive than the SPR-based optical detection where the LODs were in range of 80$100 \mathrm{nM}$ for $13-16 \mathrm{~nm}$ Au-NPs. ${ }^{150,170}$

Titration experiments were next carried out to quantitatively analyze GNP-lectin interactions. A fixed amount of the 35-nm S-M-GNP was incubated with varying concentrations of Con A, and the DLS graphs of the resulting solutions were recorded. The increases in particle diameter, D, were computed and the results plotted against the concentration of Con A (Figure 3.4a). The saturation curve was then fitted with an overall binding model, i.e., the Hill Equation (Eq. 3.3),

$$
Y=\frac{B_{\max } * X^{h}}{K_{D}^{h}+X^{h}}
$$

where $B_{\max }$ is the maximum specific binding, $K_{D}$ is the apparent dissociation constant, and $\mathrm{h}$ is the Hill coefficient. The $\mathrm{K}_{\mathrm{D}}$ value was subsequently derived as $63 \mathrm{nM}$ for SM-GNP (Table 3.1). Following the same procedure, the $K_{D}$ value of Au-M-GNP was 
determined to be $86 \mathrm{nM}$ (Table 3.1). These values represent more than 3 orders of magnitude increases in binding affinity than that of the free ligand Man with Con A $\left(\mathrm{K}_{\mathrm{d}}=470 \mu \mathrm{M}\right) .{ }^{148}$ The results are consistent with our previous studies that NPs can serve as an efficient multivalent scaffold greatly amplifying the affinity of the bound ligands with lectins. ${ }^{155}$ In all cases, the $\mathrm{h}$ values were larger than 1 , indicating a positive cooperativity of the ligands conjugated on GNPs.
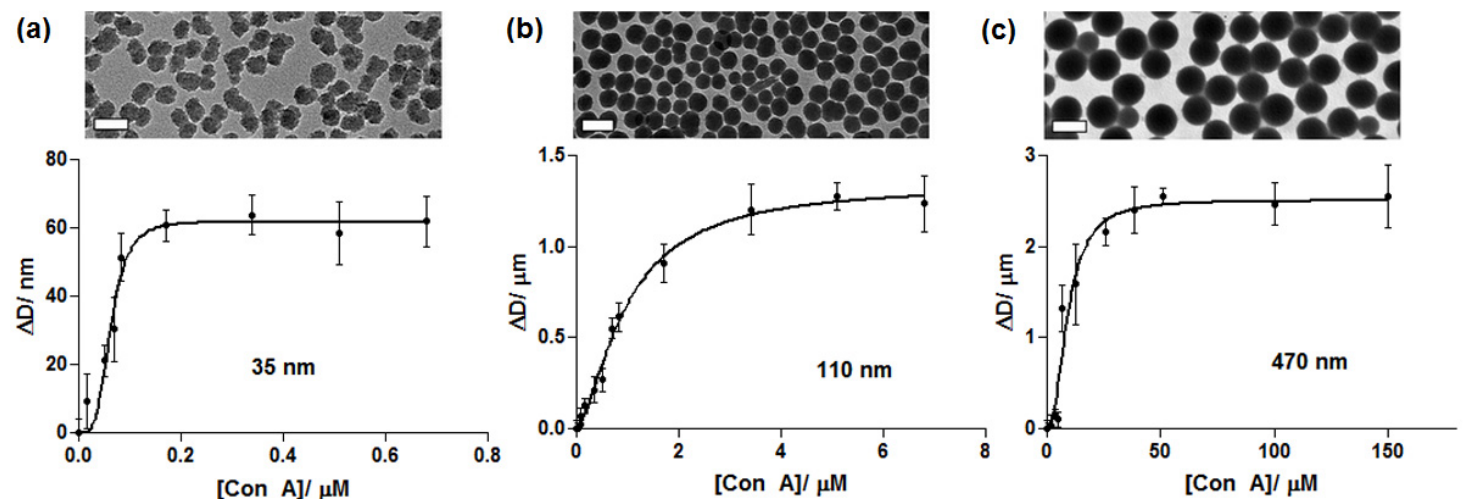

Figure 3.4 The change in particle diameter $(\Delta \mathrm{D})$ vs. lectin concentration: experimental data (circles) and the corresponding Hill fitting curves (lines) for a) $35 \mathrm{~nm}$, b) $110 \mathrm{~nm}$, and $470 \mathrm{~nm}$ silica NPs, respectively. The scale bars in the TEM images are a) $50 \mathrm{~nm}$, b) $200 \mathrm{~nm}$, and c) $500 \mathrm{~nm}$ respectively. 
Table 3.1 LOD and $K_{D}$ values of GNP-lectin interactions.

\begin{tabular}{|c|c|c|c|c|c|c|}
\hline GNP & $\begin{array}{l}\text { Diameter } \\
\text { (nm) }\end{array}$ & $\begin{array}{c}\text { Number of } \\
\text { ligand per NP }\end{array}$ & Lectin & $\begin{array}{l}\text { LOD } \\
\text { (nM) }\end{array}$ & $\mathbf{K}_{\mathbf{D}}(\mu \mathbf{M})$ & h \\
\hline \multirow{3}{*}{ S-M-GNP } & 35 & 830 & \multirow{3}{*}{ Con A } & 2.9 & 0.063 & 3.9 \\
\hline & 110 & 2600 & & 42 & 0.97 & 1.6 \\
\hline & 470 & 4900 & & $6.2 \times 10^{3}$ & 9.2 & 2.3 \\
\hline Au-M-GNP & 40 & 950 & Con A & 15 & 0.086 & 2.2 \\
\hline \multirow{2}{*}{ S-G-GNP } & 35 & 830 & Con A & \multicolumn{3}{|c|}{$N / A$} \\
\hline & 35 & 840 & $\mathrm{RCA}_{120}$ & 6.6 & 0.22 & 1.9 \\
\hline
\end{tabular}

N/A: not applicable.

\section{Impact of NP size on binding affinity}

Size-dependency has been recognized as of high significance affecting the physical and chemical properties of NPs, including adsorption, bio-affinity and catalysis. ${ }^{171-173}$ To study the impact of the particle size on the binding affinity of GNPs, silica NPs with an average diameter of 35,110 , and $470 \mathrm{~nm}$, respectively, were synthesized. Particles of $35 \mathrm{~nm}$ and $110 \mathrm{~nm}$ in size were obtained by varying the reagent concentrations using the Stöber method. ${ }^{162}$ The $470 \mathrm{~nm}$ particles were prepared using a seed-growth method. ${ }^{174}$ These particles were uniform in shape and size (see TEM images, Figure 3.4), and were more monodisperse than the Stöber particles as demonstrated by a narrower particle size distribution for the $470 \mathrm{~nm}$ nanoparticles. Man ligands were subsequently conjugated on these silica NPs following the same protocol as described above, and the ligand densities were determined by the 
colorimetric method using anthrone/ $\mathrm{H}_{2} \mathrm{SO}_{4}$. As the size of the NPs increases, the number of coupled Man increases (Table 3.1), which is expected since the bigger particle has a larger surface and can thus accommodate more ligands.

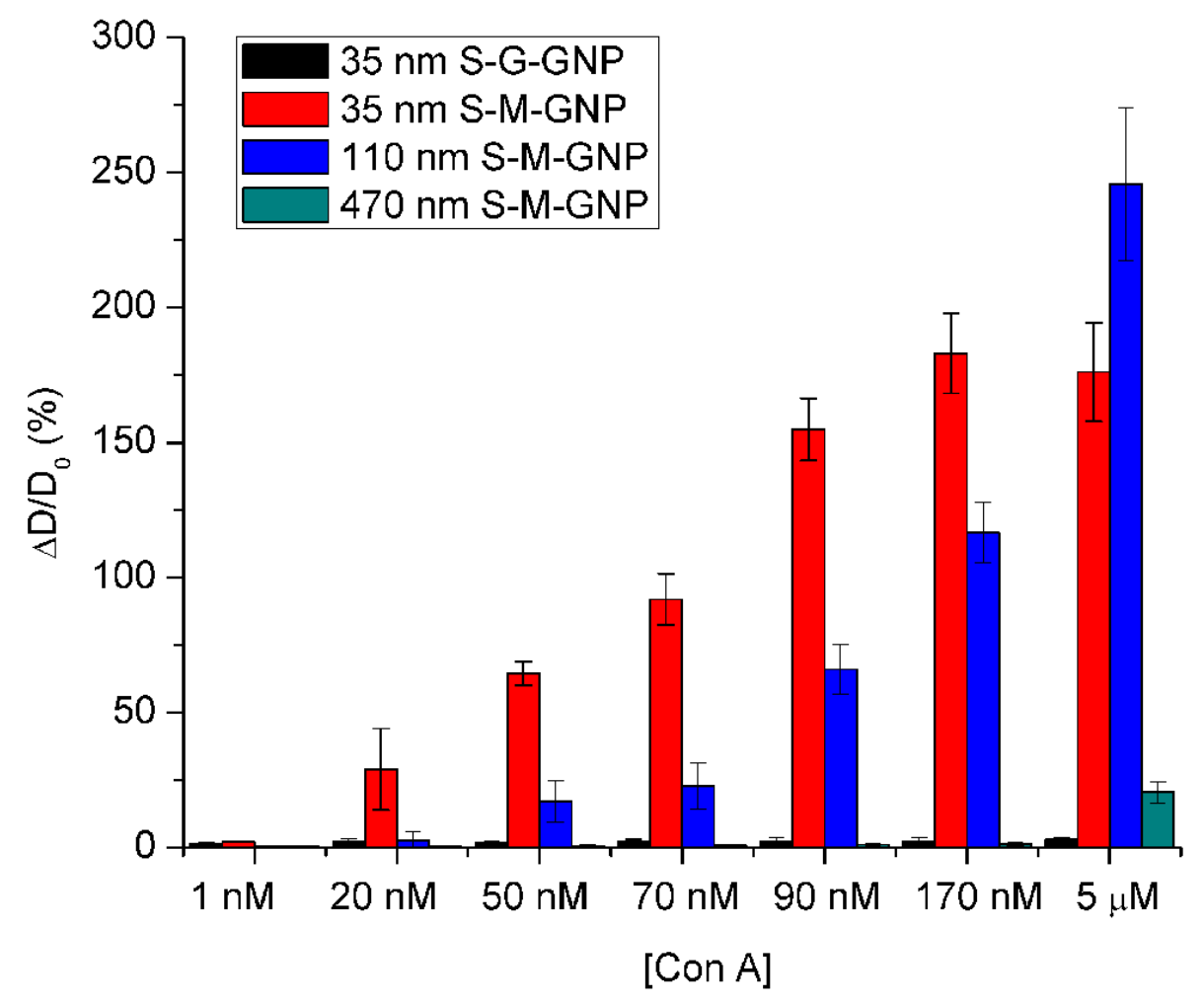

Figure 3.5 Percent increase in particle size (= increase in particle diameter $(\Delta \mathrm{D})$ /original particle diameter $\left.\left(\mathrm{D}_{0}\right) \times 100 \%\right)$ vs. concentration of Con $\mathrm{A}$ for various GNPs.

The GNPs were then treated with varying concentrations of Con A, and the percentage increase in particle diameter was calculated from the DLS measurements, shown in Figure 3.5. A noticeable change in the particle diameter was observed for the $35 \mathrm{~nm} \mathbf{S}$ M-GNP when $20 \mathrm{nM}$ Con A was added. The particle size did not change for the 110 $\mathrm{nm}$ and $470 \mathrm{~nm}$ S-M-GNP until the Con A concentration reached $50 \mathrm{nM}$ and $5 \mu \mathrm{M}$, 
respectively, indicating that smaller NPs were more sensitive in detecting GNP-lectin interactions. This is consistent with the LOD results determined for these GNPs, which were $2.9 \mathrm{nM}, 42 \mathrm{nM}$, and $6.2 \mu \mathrm{M}$ for $35 \mathrm{~nm}, 110 \mathrm{~nm}$, and $470 \mathrm{~nm}$ S-M-GNPs, respectively (Table 3.1). This size-dependent phenomenon was also observed by Huo and coworkers where $\mathrm{Au}$ NPs were used to detect DNA by DLS. ${ }^{158}$ Titration experiments were subsequently carried out on all GNPs synthesized, and the apparent $\mathrm{K}_{\mathrm{D}}$ values were determined from the saturation binding curves in the same manner as shown in Figure 3.4. For S-M-GNP, the binding affinity decreased, i.e., the $K_{D}$ values increased with increasing particle size (Table 3.1). This is in agreement with our previous studies using Au GNPs where the affinity of GNPs with lectins decreased with increasing particle size. ${ }^{155}$ A possible explanation for the lower LOD and binding affinity of the larger GNPs is the steric hindrance imposed by the larger particles. Although there are more ligands on the larger particles (Table 3.1), these ligands are less accessible to the lectin as compared to smaller particles with the same length of the spacer linker.

The DLS method is also highly specific. When Gal, a non-binding ligand for Con A, was conjugated to the 35-nm silica NPs and the resulting S-G-GNP was subsequently treated with Con A, no obvious change in particle size was observed at all Con A concentrations up to $5 \mu \mathrm{M}$ (Figure 3.5 ). S-G-GNP was then treated with $\mathrm{RCA}_{120}$, an R-type lectin exhibiting broad specificity for the terminal galactose group. ${ }^{175} \mathrm{RCA}_{120}$ is a dimer having one active Gal-binding site on each subunit. ${ }^{176}$ The lectin can 
therefore act as a crosslinker forming a complex with Gal-coated NPs. ${ }^{177}$ Indeed, when S-G-GNP was treated with $\mathrm{RCA}_{120}$, aggregates formed and the size of which could be monitored by DLS. Following the same procedure as described above, the LOD of $\mathrm{RCA}_{120}$ was measured to be $6.6 \mathrm{nM}$, which is on the same order of magnitude as that of S-M-GNP/Con A system (2.9 nM, Table 3.1). Titration experiments were then carried out, and the particle size changes were plotted against the $\mathrm{RCA}_{120}$ concentration. The apparent $\mathrm{K}_{\mathrm{D}}$ was then calculated by fitting the saturation curve with the Hill equation (Eq. 3.3). The value, $0.22 \mu \mathrm{M}$ (Table 3.1), corresponds to an affinity enhancement of over 4 orders of magnitude in comparison to that of the free Gal with $\operatorname{RCA}_{120}\left(\mathrm{~K}_{\mathrm{d}}=455 \mu \mathrm{M}\right) .{ }^{176}$

\section{Conclusions}

In summary, a new method, based on DLS, was developed to study the interactions of GNPs with lectins. The method relies on the particle size growth of GNPs, resulting from the multiple binding sites on lectins that act as crosslinkers bringing GNPs together to form larger aggregates. Two GNP-lectin systems, M-GNP with Con A and G-GNP with $\mathrm{RCA}_{120}$, were investigated and the particle size growth was observed only for the specific binding pairs. The technique is highly sensitive, and the LOD was on a par with values obtained by other techniques. Quantitative analysis is also possible from the titration experiments, from which the apparent $K_{D}$ values were obtained. The results showed that the binding affinities of GNPs with lectins were 3-4 
orders magnitude higher than that of the free ligands, demonstrating that NPs are an efficient scaffold amplifying the glycan-lectin interactions. The effect of particle size was also studied, and results demonstrate that smaller GNPs gave higher detection sensitivity as well as binding affinity. The method is applicable to both Au and silica NPs, and is therefore general regardless of the nature of the particles. The high sensitivity of the DLS method comes from the crosslinking ability of the lectins, which can be a limitation. However, many lectins contain more than 2 subunits and can act as crosslinkers inducing particle aggregation. The method developed here, coupled with the simplicity and fast measurement of the DLS technique, is therefore highly valuable in studying GNP-lectin interactions. 


\subsection{Isothermal Titration Calorimetry (ITC)}

(a)

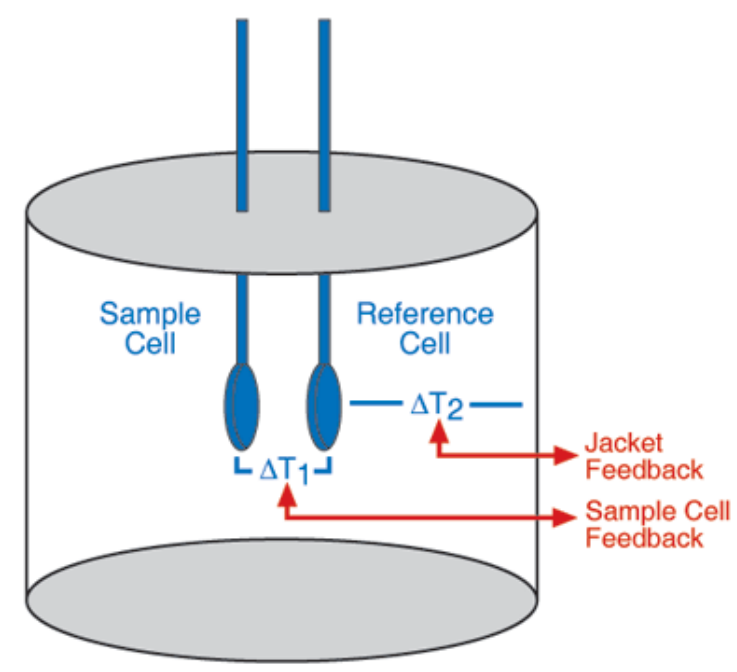

(b)

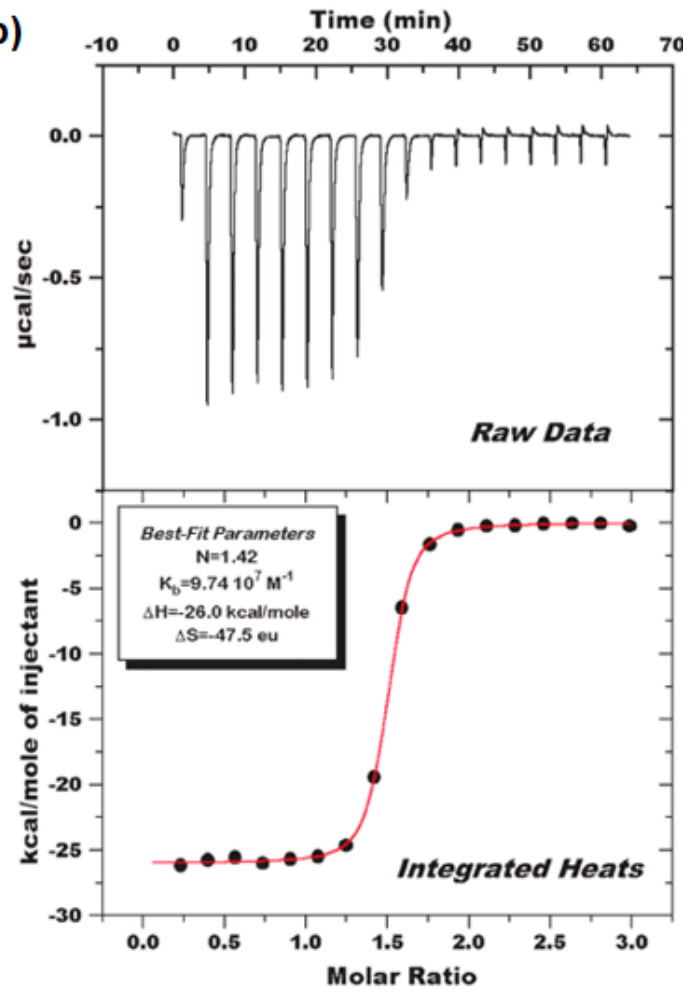

Figure 3.6 (a) Diagram of ITC cells and (b) Typical ITC data. Top: raw ITC data. Bottom: binding isotherm curve. ${ }^{178}$

In this chapter, an ITC-based method has been developed for determining the binding affinity of glyconanoparticles with lectins. ITC is a sensitive and quantitative analytical technique that determines the thermodynamic parameters of binding events occurring in solution. By measuring the heat absorbed or released upon the interactions between ligands and biomacromolecules, the binding constant $\left(\mathrm{K}_{\mathrm{a}}\right)$, enthalpy of binding $(\triangle \mathrm{H})$ and stoichiometry of binding (n) can be derived simultaneously. ${ }^{179}$ The working principle of ITC is based on heat 
measurement between a reference cell and a sample cell where the binding actually occurs (Figure 3.6a). During the experiment, the temperature of the sample and reference cell is adjusted to be the same by supplying or absorbing heat to the sample cell, and the amount of heat absorbed/released is recorded by a sensor, which is attributed to the interaction heat in sample cell. The raw data was obtained by plotting the quantity of heat versus time. Subsequently, heat was integrated as function of molar ratio between the injectant, e.g., Con A, and its binding partner, e.g. GNP-M, to give the binding curve (Figure 3.6b). Current commerical ITC instrument is capable of measuring a wide range of interactions with $\mathrm{K}_{\mathrm{d}}$ values ranging from millimolar to nanomolar. Based on the results from literature $^{11,152}$ and our previous studies, ${ }^{155,180}$ apparent dissociation constants of GNP-lectin interactions were mostly in the nanomolar range, and therefore, ITC should be a suitable method for measuring GNP-lectin interactions. ${ }^{178}$

\section{Experimental details}

Preparation of gold glyconanoparticles.

AuNPs, $22 \mathrm{~nm}$ in diameter, were prepared and functionalized with PFPA-disulfide following the same procedure as described in Chapter 2. ${ }^{155}$ The subsequent carbohydrate coupling was carried out photochemically as described in Chapter 2. ${ }^{181}$ Briefly, a solution of PFPA-functionalized AuNPs in acetone (10 nM) mixed with an aqueous solution of carbohydrate $(10 \mathrm{mM})$ was irradiated for 12 min with a 450-W medium pressure $\mathrm{Hg}$ lamp (Hanovia) with a 280-nm long-path optical filter. The 
resulting GNPs were then dialyzed overnight to remove excess carbohydrate. Before the binding experiments, the GNPs were incubated in HEPES buffer containing $0.01 \%$ Tween 20 and 3\% BSA for 1 hour, centrifuged, and placed in the buffer without BSA until further use.

ITC

ITC experiments were performed using an ITC200 microcalorimeter from Microcal, LLC. (Northampton, MA) in HEPES buffer. The concentration of lectin was $10 \mu \mathrm{M}$, and that of GNP was $2.5 \mathrm{mM}$. In each experiment, $\sim 38 \mu \mathrm{L}$ of lectin was injected through the computer-controlled $40-\mu \mathrm{L}$ micro-syringe at an injection interval of 4 min into the nanoparticle solution (cell volume $=200 \mu \mathrm{L}$ ) while stirring at $1000 \mathrm{rpm}$. The experimental data were fitted to a theoretical titration curve using the software supplied by MicroCal. A standard one-site model was used with $\Delta \mathrm{H}$ (enthalpy change, in $\mathrm{kcal} / \mathrm{mol}), \mathrm{K}_{\mathrm{a}}$ (association constant, in $\mathrm{M}^{-1}$ ), and $\mathrm{N}$ (number of binding sites) as the variables.

\section{Results and discussion}

GNPs were synthesized by coupling carbohydrates to Au NPs using the perfluorophenyl azide (PFPA) photocoupling chemistry developed in our laboratory. ${ }^{165,181} 106,140$ AuNPs of $22 \mathrm{~nm}$ in diameter were synthesized and functionalized with PFPA (see Supplementary Information for detailed synthesis). 
D-Mannose (Man), D-galactose (Gal), and $\alpha$-1,2-mannobiose (Man2) were then conjugated to the PFPA-functionalized AuNPs by light activation. ${ }^{165,181}$ The density of the attached carbohydrates were determined colorimetrically using the anthrone-sulfuric acid assay (Table 3.2). ${ }^{181}$ Binding studies were carried out using a plant lectin Concanavalin A (Con A), a well-studied tetrameric protein having specific binding site for the terminal $\alpha$-mannosyl groups. ${ }^{167,114}$ In the ITC experiments, the lectin was loaded into the micro-syringe and was titrated into the GNP solution placed in the calorimeter cell. When the binding occurs between GNPs and lectin, such as Man-conjugated GNP (GNP-Man) and Con A, heat will be released upon each injection. The amount of generated heat decreases with each subsequent injection as the GNPs in the cell are consumed with bound lectin. In contrast, if a non-binding lectin is used, the released heat, likely produced due to reagent mixing, will be small and will remain constant with each injection. 
(a)

Time $(\mathrm{min})$

(b)

Time (min)

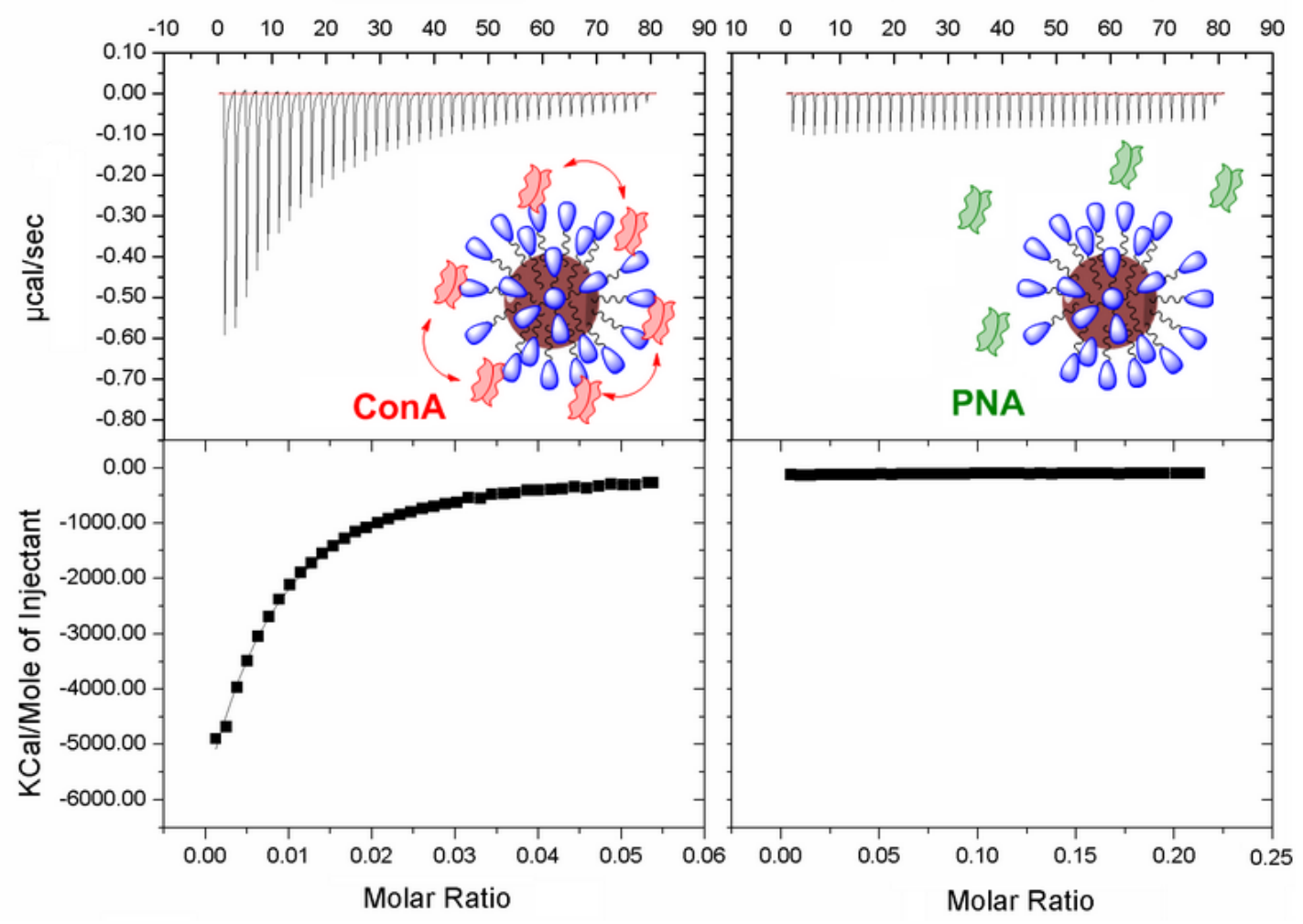

Figure 3.7 ITC graphs of GNP-Man with a) Con A and b) PNA. The experimental data (solid squares) were fit to theoretical titration curves (solid lines) using the software supplied by the ITC manufacturer.

Figure $3.7 \mathrm{a}$ is a typical ITC titration graph of GNP-Man with Con A at $\mathrm{pH} 7.6$ in HEPES buffer containing $1 \mathrm{mM} \mathrm{Mn}^{2+}$ and $1 \mathrm{mM} \mathrm{Ca}^{2+}$. As expected, heat was generated after each injection of the lectin solution, and the amount of the released heat decreased gradually with each additional injection. Because heat can also be produced just by mixing the reagents, background heat from dilutions and was substracted from each titration experiment. Figure 3.7a (bottom) shows the 
processed titration data (solid squares) and the computed integrated curve (line) which fit the data points well. The thermodynamic parameters, including dissociation constant $\left(K_{D}\right)$, the enthalpy change $(\Delta H)$, the entropy change $(\Delta S)$, and the number of binding sites (n) are shown in Table 3.2. The Gibbs free energy changes $(\Delta \mathrm{G})$ was calculated by standard thermodynamic equation: $\Delta \mathrm{G}=\Delta \mathrm{H}-\mathrm{T} \Delta \mathrm{S}$. The enhancement factor $\beta$, defined as $\log \left(\mathrm{K}_{\mathrm{d}} / \mathrm{K}_{\mathrm{D}}\right)$, can be used to evaluate the multivalency effect when nanoparticles are used as the scaffolds. ${ }^{69,182}$ Compared to the binding affinity of the free $\alpha-\mathrm{D}$-mannopyranoside ligand with Con $\mathrm{A}\left(\mathrm{K}_{\mathrm{d}}=470\right.$ $\mu \mathrm{M}),{ }^{136}$ the $\mathrm{K}_{\mathrm{D}}$ value of GNP-Man with Con $\mathrm{A}\left(\mathrm{K}_{\mathrm{D}}=122 \mathrm{nM}\right)$ was closed to 4 orders of magnitude smaller, i.e. the affinity was 4 orders of magnitude higher. The number of binding sites, $N(=1 / n)$, represents the number of sites on the nanoparticle that were available for binding with the lectin. Note that $\mathrm{N}(306)$ is smaller than the number of carbohydrate ligands immobilized on the particle surface (3600), meaning that only a portion of available ligands on the GNP participated in binding the lectin. This is expected considering that the distance between each of the four monomeric binding site on Con A is $6.5 \mathrm{~nm},{ }^{183}$ which is far greater than the distance between Man ligands on the GNP surface $(2 \sim 3 \mathrm{~nm}){ }^{155}$ The results indicated the binding of GNP wih lectin is exothermic process $(\Delta \mathrm{H}<0$ and $\Delta \mathrm{S}<0)$, and an overall negative Gibbs free energy gain $(\Delta \mathrm{G})$ was obtained due to the slight difference between unfavorable entrophy and enthalpy changes. In the control experiment where PNA lectin was titrated into GNP-Man, the released heat was small and remained unchanged throughout the titration (top, Fig. 1b). 
Subsequent data fitting gave a flat titration curve (bottom, Fig. 1b). Similar results were also obtained when Con A was titrated into GNP-Gal where galactose is a non-binding ligand for Con A. These results demonstrated that there was no apparent binding between the non-interacting GNP-lectin pairs.

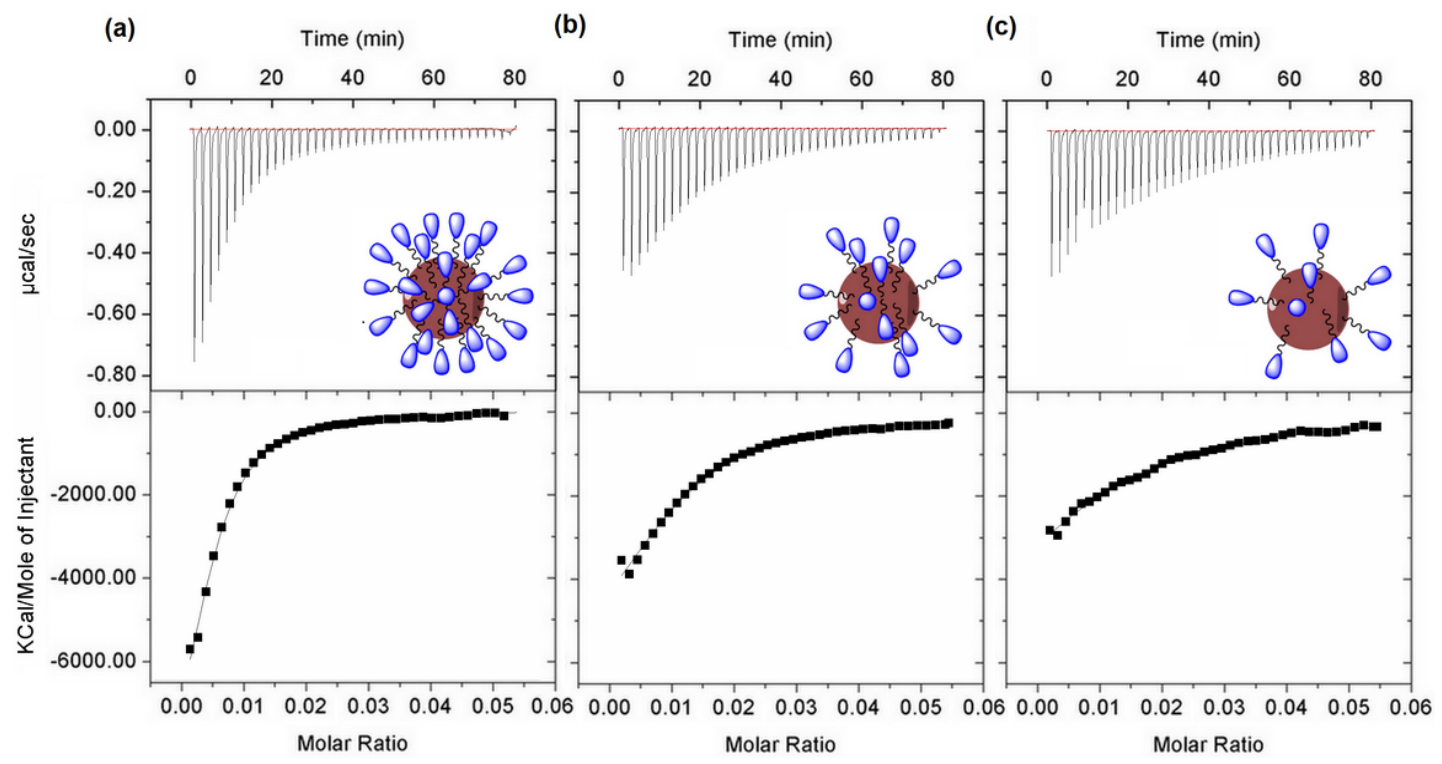

Figure 3.8 ITC titration graphs of Con A with (a) GNP-Man2I, (b) GNP-Man2II, and (c) GNP-Man2III. Solid squares: experimental data, lines: fitted titration curves.

To study the effect of ligand density on lectin binding, ITC experiments were carried out where the ligand density on GNP-Man2 was varied from 1400 (GNPMan2I, Figure 3.8a,), to 260 (GNP-Man2II, Figure 3.8b) and 110 (GNP-Man2III, Figure 3.8c), which were prepared following a mixed ligand strategy. ${ }^{155}$ GNP-M2I, having the highest ligand density, gave the largest $\mathrm{N}$ value and the highest binding affinity (Table 3.2). Although GNP-M2I has 5 times and 13 times more ligands than GNP-M2III and GNP-M2III, respectively, the binding affinity was only 2 
times and 5 times larger. This may be attributed to the fact that not all ligands participated in binding the lectin. ${ }^{155}$ GNP-M2III had a slightly smaller N value than GNP-M2II. However, it gave a slightly more favorable thermodynamic values ( $\Delta \mathrm{H}$ and $\Delta \mathrm{G}$ ) probably due to the reduced steric hindrance at lower ligand density. The method was further tested with a bacterial lectin, cyanovirin-N (CV-N). ${ }^{184-187}$ GNP-Man2 was used since Man2 is an epitope ligand for CV-N. Mut ${ }^{\mathrm{DB}}$, a variant of CV-N and having only one active domain ${ }^{188}$ (instead of two in the wide-type CV-N), was used in this study. Results from the ITC titration curves (Figure 3.9) show 4.6 orders of magnitude affinity enhancement $(\beta)$ of GNP-Man2I over free Man2 to CV-N (Table 3.2), similar to the results obtained previously using a fluorescent competition assay. ${ }^{189}$ 


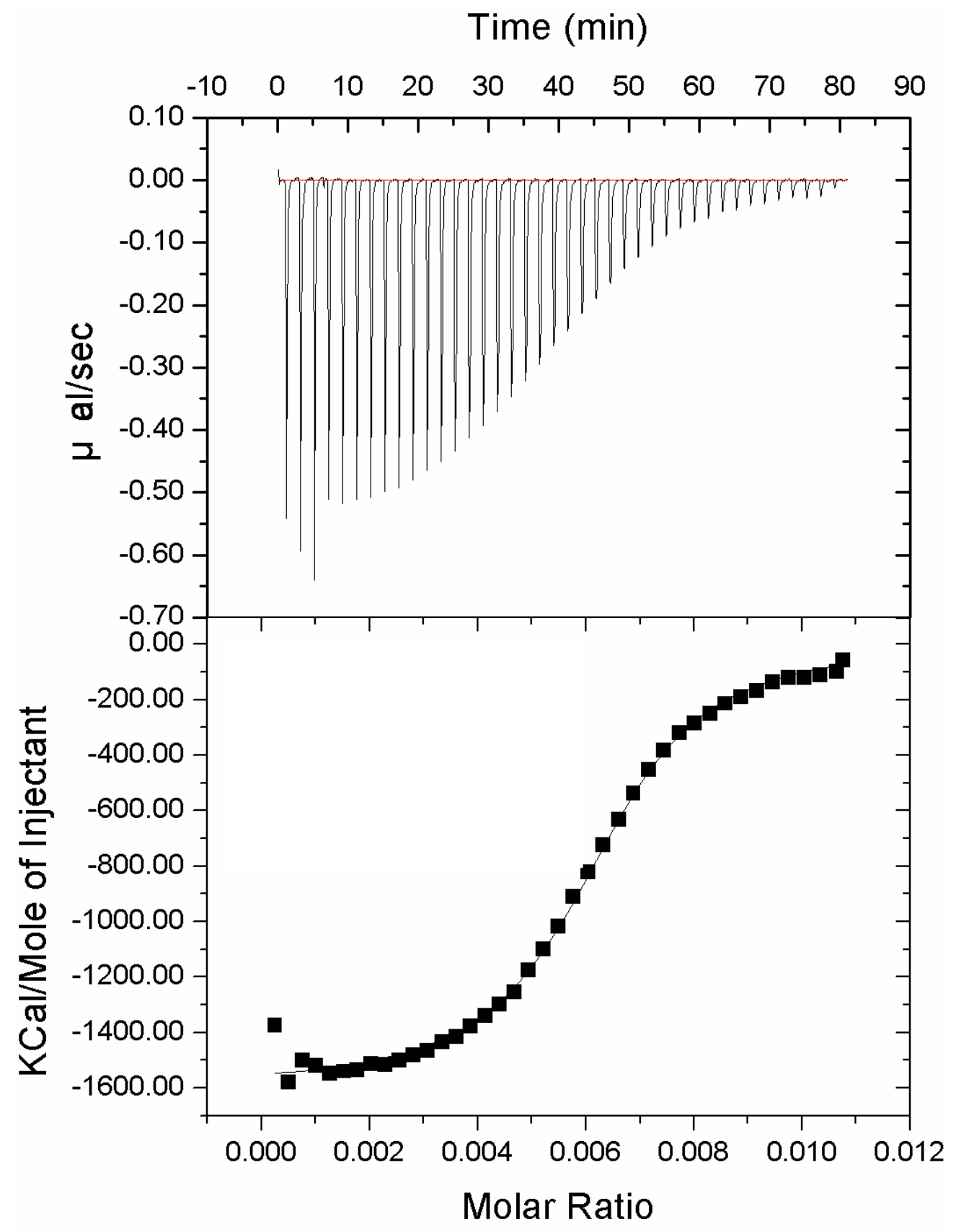

Figure 3.9 ITC titration graphs of GNP-Man2 with CV-N Mut ${ }^{\mathrm{DB}}$ 
Table 3.2 The thermodynamic parameters of GNP-lectin interactions at $25{ }^{\circ} \mathrm{C}$.

\begin{tabular}{|c|c|c|c|c|c|c|c|c|c|c|c|}
\hline \multicolumn{2}{|c|}{ GNP } & $\begin{array}{c}\text { Ligand } \\
\text { density } \\
\text { (per } \\
\text { NP) }\end{array}$ & Lectin & $\begin{array}{l}\mathrm{K}_{\mathrm{D}} / \\
\mathrm{nM}\end{array}$ & $\begin{array}{l}\mathrm{K}_{\mathrm{d}} / \\
\mu \mathrm{M}\end{array}$ & $\beta^{\mathrm{a}}$ & $\begin{array}{c}-\Delta \mathrm{G} / \\
\mathrm{kJ} \mathrm{mol} \\
1\end{array}$ & $\begin{array}{c}\Delta \mathrm{H} / \mathrm{kJ} \\
\mathrm{mol}^{-1}\end{array}$ & $\begin{array}{c}\mathrm{T} \Delta \mathrm{S} / \mathrm{kJ} \\
\mathrm{mol}^{-1}\end{array}$ & $\mathrm{n}$ & $\mathrm{N}^{\mathrm{b}}$ \\
\hline \multirow{2}{*}{\multicolumn{2}{|c|}{$\begin{array}{l}\text { GNP- } \\
\text { Man }\end{array}$}} & \multirow{2}{*}{3600} & Con $\mathrm{A}$ & 122 & 470 & 3.6 & 137 & -107760 & -107623 & 0.00327 & 306 \\
\hline & & & PNA & \multicolumn{8}{|c|}{$\mathrm{ND}^{\mathrm{c}}$} \\
\hline \multicolumn{2}{|c|}{$\begin{array}{c}\text { GNP- } \\
\text { Gal }\end{array}$} & 3500 & Con $\mathrm{A}$ & \multicolumn{8}{|c|}{$\mathrm{ND}^{\mathrm{c}}$} \\
\hline \multirow{3}{*}{$\begin{array}{l}\text { GNP- } \\
\text { Man2 }\end{array}$} & I & 1400 & \multirow{3}{*}{ Con A } & 34.8 & \multirow{3}{*}{24} & 2.8 & 18.3 & -46356 & -46338 & 0.00478 & 209 \\
\hline & II & 260 & & 82.6 & & 2.5 & 6.5 & -28407 & -28401 & 0.0101 & 99 \\
\hline & III & 110 & & 175 & & 2.1 & 59.2 & -30577 & -30518 & 0.0121 & 83 \\
\hline \multicolumn{2}{|c|}{$\begin{array}{l}\text { GNP- } \\
\text { Man2 }\end{array}$} & 1400 & $\mathrm{CV}-\mathrm{N}$ & 17.2 & $757^{188}$ & 4.6 & 47.1 & -6650 & -6602 & 0.00625 & 160 \\
\hline
\end{tabular}

a enhancement factor: $\beta=\log \left(\mathrm{K}_{\mathrm{d}} / \mathrm{K}_{\mathrm{D}}\right)$

${ }^{b}$ number of binding site on GNP: $N=1 / n$ (n: number of binding sites on lectin) c not detectable

In conclusion, an ITC-based method has been developed to quantitatively analyze the binding affinity of glyconanoparticles with lectins. Thermodynamic parameters including dissociation constant, entropy and enthalpy changes, and the number of binding site were simultaneously determined by titrating GNPs into the lectin solution. A 3-5 orders of magnitude affinity enhancement over the free ligand was observed, which could be attributed to the multivalent ligand presentation on 
nanoparticles. The results were consistent with what have been obtained previously using other analytical techniques such as fluorescence competition assays, dynamic light scattering, and microarrays. In contract to these methods that either require a label, or rely on indirect information, ITC is a direct measurement method that gives the affinity constant, themodynamic parameters and stoichimetry of the interactions. This technique provides a general and highly sensitive means to study nanomaterial interactions with biological entities. The method developed will benefit fundamental studies as well as the development of biosensors and theranostic tools. 


\section{Chapter 4. Impact of Ligand Presentation on the Binding Affinity of Glyconanoparticles - Multivalency Effect}

This work was published on "Wang, X.; Ramström, O.; Yan, M., Quantitative analysis of Multivalent Ligands Presentation on Gold Glyconanoparticles and Their Effects on Protein Binding. Anal. Chem. 2010, 82 (21), 9082-9089”

http://pubs.acs.org/doi/abs/10.1021/ac102114z

Reproduced by permission of American Chemical Society 


\section{Introduction}

A general feature of carbohydrate-protein interactions relates to their inherent complexity, and multivalent ligand expression is frequently used to achieve sufficiently high affinities required for sensitive recognition. ${ }^{68,69,190}$ In analogy to ligand clustering at cell surfaces, nanomaterials can function as efficient nano-sized scaffolds for multiple carbohydrate presentation. Strong binding enhancements resulting from ligand presentation at the surfaces of nanomaterials have been demonstrated in numerous studies. ${ }^{191-193}$ However, the current investigations are generally short of in-depth characterization of the structures, compositions and densities of the surface ligands, as well as the biological activities of the constructs, since conventional surface analysis methods have been less optimized for nanomaterials. Owing to the increasing interest in nanomaterials research, recent development in spectroscopic and microscopic techniques has afforded detailed structural and compositional information for nanomaterials. ${ }^{137,194,195}$ Nevertheless, quantitative analysis of multivalent biological affinity at nanoparticle surfaces still represents a considerable challenge. Furthermore, very limited methods were available to measure the binding affinity of glyconanoparticles with proteins. ${ }^{11,196,197}$

In this chapter, we present detailed quantitative analyses on nanoparticle-based multivalent carbohydrate-protein interactions. The effects resulting from the nanoparticle size was investigated, and the ligand presentation with respect to ligand density, surface environment, and linker spacer was studied. The well-established 
interaction system involving the lectin Con A, together with a panel of carbohydrate species, was chosen as the target. This lectin is present as a tetramer at $\mathrm{pH}>7$, and possesses specific affinities to $\alpha$-D-mannopyranoside, $\alpha$-D-glucopyranoside and their derivatives. ${ }^{198,199}$ The system constitutes an excellent model for investigating the multivalency effect, ${ }^{114}$ and studies on mannose-Con A interactions have established that multivalent effects are highly sensitive to a range of factors, including the number of binding sites, the ligand density, the structure of the ligand linkers, and the coupling chemistry of the ligand attachments. ${ }^{200-203}$ In the present study, a recently developed photochemical carbohydrate immobilization technique was adopted, ${ }^{154,181}$ and an array of GNPs functionalized with different mono-, oligo-, and poly-saccharides were prepared and ligand densities determined. A fluorescence-based ligand competition assay was employed for the quantitative analysis of the binding affinities of the GNPs where the apparent $K_{d}$ values of the resulting GNPs were determined. The binding affinity of GNPs with respect to the ligand density, spacer length, linker structure, as well as the nanoparticle size was furthermore investigated.

\section{Experimental details}

\section{Synthesis of PFPA-Thiols}


HOt ${ }_{4}+\gamma_{9} \mathrm{SH}$

1a

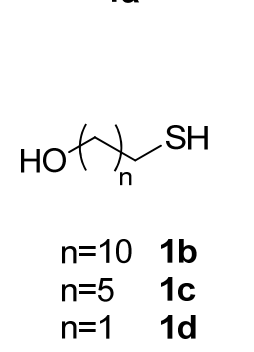<smiles>CC(C)SCC(C)C(C)(C)OCCO</smiles>

$\mathrm{I}_{2}$
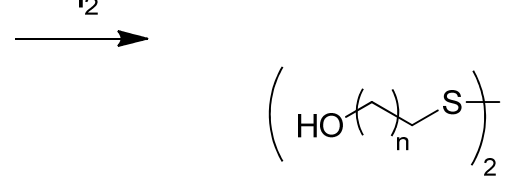<smiles>Nc1c(F)c(F)c(C(=O)O)c(F)c1F</smiles>

DMAP, EDAC<smiles>CC(C)(C)COCCOC(=O)c1c(F)c(F)c(N)c(F)c1F</smiles><smiles>CC(C)(C)OCCOC(=O)c1c(F)c(F)c(N)c(F)c1F</smiles><smiles>CC(C)SCCOC(=O)c1c(F)c(F)c(N)c(F)c1F</smiles>

$\begin{array}{cc}n=10 & \mathbf{2 b} \\ n=5 & \mathbf{2 c} \\ n=1 & \mathbf{2 d} \\ & \\ & \end{array}$<smiles>CC(S)COC(=O)c1c(F)c(F)c(N)c(F)c1F</smiles>

$\begin{array}{ll}n=10 & b \\ n=5 & c \\ n=1 & d\end{array}$

Figure 4.1 Synthesis of PFPA-thiols a-d.

\section{3,6,9,12,37,40,43,46-Octaoxa-24,25-dithiaoctatetracontane-1,48-diylbis(4-azido-}

2,3,5,6-tetrafluorobenzoate) (2a) (1-Mercaptoundec-11-yl)tetra(ethylene glycol) (1a, $100 \mathrm{mg}, 0.26 \mathrm{mmol})$ in absolute ethanol $(10 \mathrm{~mL})$ was titrated with a saturated solution of iodine in ethanol until the brown color of iodine persisted. The solution was concentrated to $2 \mathrm{~mL}$ and then water $(10 \mathrm{~mL})$ was added. The solution was extracted with diethyl ether $(3 \times 10 \mathrm{~mL})$. The combined ethereal extracts were washed with brine, dried over $\mathrm{Na}_{2} \mathrm{SO}_{4}$, and the solvent was removed under reduced pressure to afford the disulfide as a brown oil. A solution of 4-azido-2,3,5,6-tetrafluorobenzoic 
acid ${ }^{129}(61.8 \mathrm{mg}, 0.26 \mathrm{mmol})$ in $\mathrm{CH}_{2} \mathrm{Cl}_{2}(10 \mathrm{~mL})$ was cooled to $0{ }^{\circ} \mathrm{C}$, and DMAP (3.7 $\mathrm{mg}, 0.03 \mathrm{mmol})$ and EDAC (57.5 $\mathrm{mg}, 0.3 \mathrm{mmol})$ were added. The disulfide obtained above was then added, and the solution was stirred for 1 hour, after which the reaction was allowed to warm up to room temperature and was stirred for $12 \mathrm{~h}$ under argon. The product was recovered by extracting with $\mathrm{CH}_{2} \mathrm{Cl}_{2}$ and the organic layer was washed with water, brine, and dried over $\mathrm{Na}_{2} \mathrm{SO}_{4}$. The crude product was purified by column chromatography with 1:8 v/v hexanes/ethyl acetate to afford PFPA-disulfide 2a as a clear oil (57.1 mg, 37\%). ${ }^{1} \mathrm{H}$ NMR (400 MHz, $\left.\mathrm{CDCl}_{3}\right): \delta(\mathrm{ppm}) 4.52(\mathrm{t}, J=3.2$ Hz, 4H), $3.81(\mathrm{t}, J=3.2 \mathrm{~Hz}, 4 \mathrm{H}), 3.66-3.54(\mathrm{~m}, 24 \mathrm{H}), 3.46(\mathrm{t}, J=5.2 \mathrm{~Hz}, 4 \mathrm{H}), 2.67(\mathrm{t}$, $J=7.2 \mathrm{~Hz}, 4 \mathrm{H}), 1.61(\mathrm{~m}, 4 \mathrm{H}), 1.57(\mathrm{~m}, 4 \mathrm{H}), 1.28-1.23(\mathrm{~m}, 28 \mathrm{H}) .{ }^{13} \mathrm{C} \mathrm{NMR}(100$ $\left.\mathrm{MHz}, \mathrm{CDCl}_{3}\right): \delta(\mathrm{ppm}) 159.4,145.4(\mathrm{~d}, J=260 \mathrm{~Hz}), 140.2(\mathrm{~d}, J=252 \mathrm{~Hz}), 127.9$ $109.5,70.4,69.6,69.1,68.4,61.3,37.4,36.7,30.4,30.1,29.3,29.2,28.4,28.0$.

\section{3-Mercapto-3,6,9,12-tetraoxatricosyl 4-azido-2,3,5,6-tetrafluorobenzoate (a).}

To a solution of PFPA-disulfide 2a $(40 \mathrm{mg}, 0.033 \mathrm{mmol})$ in $1: 1 \mathrm{v} / \mathrm{v}$ ethanol/acetonitrile $(20 \mathrm{~mL})$, zinc dust $(50 \mathrm{mg})$ and concentrated $\mathrm{HCl}(0.05 \mathrm{~mL})$ were added, and the reaction mixture was stirred under argon at room temperature for 1 hour. The reaction mixture was filtered, and the solvents were removed by rotary evaporation. The residue was dissolved in chloroform, and the resulting solution was washed twice with water followed by dilute $\mathrm{NaHCO}_{3}$ solution, and was dried over $\mathrm{Na}_{2} \mathrm{SO}_{4}$. The crude product was purified by column chromatography with 1:8 $\mathrm{v} / \mathrm{v}$ hexanes/ethyl acetate to afford PFPA-thiol a as a clear oil (32.4 mg, 81\%). ${ }^{1} \mathrm{H}$ NMR $\left(400 \mathrm{MHz}, \mathrm{CDCl}_{3}\right): \delta(\mathrm{ppm}) 4.40(\mathrm{t}, J=2.8 \mathrm{~Hz}, 2 \mathrm{H}), 3.75(\mathrm{t}, J=2.6 \mathrm{~Hz}, 2 \mathrm{H}), 3.65-$ 
$3.52(\mathrm{~m}, 12 \mathrm{H}), 3.45(\mathrm{t}, J=5.6 \mathrm{~Hz}, 2 \mathrm{H}), 2.40(\mathrm{q}, J=7.6 \mathrm{~Hz}, 2 \mathrm{H}), 1.67(\mathrm{~m}, 4 \mathrm{H}) 1.27-$ $1.23(\mathrm{~m}, 15 \mathrm{H}) .{ }^{13} \mathrm{C}$ NMR (100 MHz, $\left.\mathrm{CDCl}_{3}\right): \delta(\mathrm{ppm}) 159.1,145.3(\mathrm{~d}, J=255 \mathrm{~Hz})$, $140.4(\mathrm{~d}, J=252 \mathrm{~Hz}), 128.2,108.9,70.5,69.9,69.1,68.5,61.8,37.7,30.3,29.7,29.2$, 28.7, 28.1, 23.4. IR (film) 2925, 2854, 2129, 1737, 1648, 1488, 1258, 1119, $998 \mathrm{~cm}^{-1}$. HRMS (ESI) $\mathrm{C}_{26} \mathrm{H}_{39} \mathrm{~F}_{4} \mathrm{~N}_{3} \mathrm{O}_{6} \mathrm{~S}[\mathrm{M}+\mathrm{H}]^{+}$calcd 598.2574, found 598.2535.

11,11'-Disulfanediylbi(undecane-11,1-diyl) bis(4-azido-2,3,5,6-tetrafluorobenzoate) (2b). Compound $\mathbf{2 b}$ was prepared following a previously reported procedure. ${ }^{181}$

11-Mercaptoundecyl 4-azido-2,3,5,6-tetrafluorobenzoate (b). Compound b was synthesized from $\mathbf{2 b}$ following the same procedure as described above for $\mathbf{a} .{ }^{1} \mathrm{H}$ NMR (400 MHz, $\left.\mathrm{CDCl}_{3}\right): \delta(\mathrm{ppm}) 4.36(\mathrm{t}, J=6.6 \mathrm{~Hz}, 2 \mathrm{H}), 2.52(\mathrm{q}, J=7.6 \mathrm{~Hz}, 2 \mathrm{H}), 1.72(\mathrm{~m}$,

$2 \mathrm{H}), 1.61(\mathrm{~m}, 2 \mathrm{H}), 1.44-1.20(\mathrm{~m}, 15 \mathrm{H}) .{ }^{13} \mathrm{C}\left(100 \mathrm{MHz}, \mathrm{CDCl}_{3}\right) \delta(\mathrm{ppm}) 159.5,145.1$ $(\mathrm{d}, J=252 \mathrm{~Hz}), 140.2(\mathrm{~d}, J=256 \mathrm{~Hz}) 125.3,108.1,64.3,36.1,29.0,28.1,25.4,23.7$. Anal. Calcd for $\mathrm{C}_{18} \mathrm{H}_{23} \mathrm{~F}_{4} \mathrm{~N}_{3} \mathrm{O}_{2} \mathrm{~S}$ : C, 51.30; H, 5.50; N, 9.97. Found C, 51.36; H, 5.51; N, 9.99 .

\section{6,6'-Disulfanediylbis(hexane-6,1-diyl) bis(4-azido-2,3,5,6-tetrafluoro-benzoate)}

(2c). Compound 2c was synthesized from 1c following the same procedure as described above for 2a. ${ }^{1} \mathrm{H}$ NMR $\left(400 \mathrm{MHz}, \mathrm{CDCl}_{3}\right) \delta(\mathrm{ppm}) 4.37(\mathrm{t}, J=6.4 \mathrm{~Hz}, 2 \mathrm{H})$, $2.69(\mathrm{t}, J=7.2 \mathrm{~Hz}, 2 \mathrm{H}), 1.81-1.65(\mathrm{~m}, 4 \mathrm{H}), 1.53-1.38(\mathrm{~m}, 4 \mathrm{H}) .{ }^{13} \mathrm{C}$ NMR $(100 \mathrm{MHz}$, $\left.\mathrm{CDCl}_{3}\right): \delta(\mathrm{ppm}) 159.7,145.1(\mathrm{~d}, J=256 \mathrm{~Hz}), 140.5(\mathrm{~d}, J=258 \mathrm{~Hz}), 124.4,107.2$, 64.8, 39.1, 36.4, 29.1, 28.4, 23.1. Anal. Calcd for $\mathrm{C}_{26} \mathrm{H}_{24} \mathrm{~F}_{8} \mathrm{~N}_{6} \mathrm{O}_{4} \mathrm{~S}_{2}$ : C, 44.57; H, 3.45; N, 12.00. Found C, 44.63; H, 3.50; N, 11.96. 
6-Mercaptohexyl 4-azido-2,3,5,6-tetrafluorobenzoate (c). Compound c was synthesized from $2 \mathbf{c}$ following the same procedure as described above for $\mathbf{a} .{ }^{1} \mathrm{H}$ NMR $\left(400 \mathrm{MHz}, \mathrm{CDCl}_{3}\right): \delta(\mathrm{ppm}) 4.32(\mathrm{t}, J=6.4 \mathrm{~Hz}, 2 \mathrm{H}), 2.54(\mathrm{q}, J=7.6 \mathrm{~Hz}, 2 \mathrm{H}), 1.75$ $(\mathrm{m}, 2 \mathrm{H}), 1.64(\mathrm{~m}, 2 \mathrm{H}), 1.45(\mathrm{~m}, 4 \mathrm{H}),(\mathrm{t}, J=7.8 \mathrm{~Hz}, 1 \mathrm{H}) .{ }^{13} \mathrm{C}$ NMR $(100 \mathrm{MHz}$ $\left.\mathrm{CDCl}_{3}\right): \delta(\mathrm{ppm}) 159.3,145.2(\mathrm{~d}, J=250 \mathrm{~Hz}), 140.4(\mathrm{~d}, J=266 \mathrm{~Hz}), 123.1,108.1$, 64.2, 36.1, 29.0, 28.1, 25.4, 23.7. Anal. Calcd for $\mathrm{C}_{13} \mathrm{H}_{13} \mathrm{~F}_{4} \mathrm{~N}_{3} \mathrm{O}_{2} \mathrm{~S}: \mathrm{C}, 44.44 ; \mathrm{H}, 3.73$; N, 11.96. Found C, 44.31; H, 3.75; N, 12.04.

\section{2,2'-Disulfanediylbis(ethane-2,1-diyl) bis(4-azido-2,3,5,6-tetrafluoro-benzoate)}

(2d). Compound 2d was prepared following a previously reported procedure. ${ }^{204}$

2-Mercaptoethyl 4-azido-2,3,5,6-tetrafluorobenzoate (d). Compound d was synthesized from 2d following the same procedure as described for a. ${ }^{1} \mathrm{H}$ NMR (400 $\left.\mathrm{MHz}, \mathrm{CDCl}_{3}\right): \delta(\mathrm{ppm}) 4.45(\mathrm{t}, J=6.4 \mathrm{~Hz}, 2 \mathrm{H}), 2.86(\mathrm{q}, J=7.2 \mathrm{~Hz}, 2 \mathrm{H}), 1.63(\mathrm{t}, J=$ $8.8 \mathrm{~Hz}, 1 \mathrm{H}) ;{ }^{13} \mathrm{C} \mathrm{NMR}\left(100 \mathrm{MHz}, \mathrm{CDCl}_{3}\right): \delta(\mathrm{ppm}) 159.1,145.3(\mathrm{~d}, J=258 \mathrm{~Hz})$, $140.5(\mathrm{~d}, J=267 \mathrm{~Hz}), 123.7,107.2,64.0,23.3$. Anal. Calcd for $\mathrm{C}_{9} \mathrm{H}_{5} \mathrm{~F}_{4} \mathrm{~N}_{3} \mathrm{O}_{2} \mathrm{~S}: \mathrm{C}$, 36.62; H, 1.71; N, 14.23. Found C, 36.25; H, 1.77; N, 14.02.

\section{Preparation of carbohydrate-conjugated gold nanoparticles.}

Citrate-protected $\mathrm{Au} \mathrm{NPs}, \sim 22 \mathrm{~nm}$ in diameter, were prepared by adding sodium citrate $(1 \mathrm{wt} \%, 1.8 \mathrm{~mL})$ to a boiling solution of $\mathrm{HAuCl}_{4}(0.25 \mathrm{mM}, 100 \mathrm{~mL})$ under vigorous stirring, and the boiling was continued for an additional $5 \mathrm{~min}$ when the solution became purple and finally light red. ${ }^{164}$ PFPA-thiol 3a-3d were synthesized following the procedures in Figure 4.1. In a 250-mL flask, the Au NP solution 
prepared above $(50 \mathrm{~mL})$ was mixed with a solution of PFPA-thiol in ethanol $(2.5 \mathrm{mM}$, $50 \mathrm{~mL}$ ), and the resulting solution was stirred for $3 \mathrm{~h}$. The solution was centrifuged at $10,000 \mathrm{rpm}$ for $15 \mathrm{~min}$, and the solid obtained was re-dispersed in ethanol $(10 \mathrm{~mL})$. The centrifugation and re-dispersion process was repeated for 3 times to remove excess PFPA-thiol, and the resulting PFPA-functionalized Au NPs was finally dispersed in acetone $(10 \mathrm{~mL})$. The concentration of the Au NP solution, about $10 \mathrm{nM}$, was determined by drying the sample under reduced pressure for $3 \mathrm{~h}$ and weighing. The carbohydrate coupling was carried out following the procedure reported previously. ${ }^{181}$ A solution of PFPA-functionalized Au NPs in acetone $(10 \mathrm{~mL})$ was placed in a flat-bottomed dish, and an aqueous solution of carbohydrate $(1 \mathrm{mM}, 0.5$ $\mathrm{mL}$ ) was added. The mixture was covered with a 280 -nm long-path optical filter (WG280, Schott Glass) and was irradiated with a 450-W medium pressure Hg lamp (Hanovia) for 10 min under vigorous stirring. Centrifugation of the solution at 12,000 rpm for 15 min separated the carbohydrate-attached Au NPs as precipitates. Excess carbohydrate was removed by membrane dialysis in water for 24 hours. Before binding experiments, the nanoparticles were incubated in the $\mathrm{pH} 7.4$ PBS buffer solution containing $0.01 \%$ Tween 20 and $3 \%$ BSA for $30 \mathrm{~min}$, centrifuged, and incubated in a pH 7.4 PBS solution without BSA for further use. ${ }^{204}$

\section{Determination of carbohydrate ligand density on GNPs.}

A previously-developed colorimetry method was used to determine the ligand density on $\mathrm{Au} \mathrm{NPs}{ }^{181}$ Calibration curves were obtained for each carbohydrate where 
carbohydrate solutions of various concentrations were incubated with anthrone/sulfuric acid and the absorbances at $620 \mathrm{~nm}$ were measured. Carbohydrates coupled on nanoparticles were subjected to the same assay where solutions of the GNPs in Milli-Q water $(30-50 \mu \mathrm{g} / 0.5 \mathrm{~mL})$ were treated with anthrone $/ \mathrm{H}_{2} \mathrm{SO}_{4}$. Background absorption due to Au NPs themselves was accounted for by treating citrate-protected Au NPs solution of the same concentration with anthrone $/ \mathrm{H}_{2} \mathrm{SO}_{4}$, and the absorbance at $620 \mathrm{~nm}$ was subtracted from that of the GNPs. The amount of surface-bound carbohydrate was then computed from the corresponding calibration curve.

Preparation of GNPs of varied carbohydrate density.

Mixed thiol solutions were prepared from PFPA-thiol and 1-hexanethiol in ethanol, with the mole percentage of PFPA-thiol varying from $10 \%$ to $98 \%$. The functionalization of $\mathrm{Au}$ NPs with mixed thiols followed the general functionalization procedure described above, except that the pure thiol solution was replaced by the mixed thiol solution when treating citrate-protected $\mathrm{Au}$ NPs. The subsequent carbohydrate coupling was carried out following the same procedure described in detail above.

Fluorescence competition binding assay.

AuNP-a-Man solutions of various concentrations $\left(1 \times 10^{-8}-5 \mathrm{nM}\right)$ were prepared from the stock solution (10 nM). The Con A-FITC solution (190 nM) was prepared in 
pH 7.4 PBS buffer containing $\mathrm{MnCl}_{2}(1 \mathrm{mM})$ and $\mathrm{CaCl}_{2}(1 \mathrm{mM})$. To the AuNP-aMan solution $(1 \mathrm{~mL})$ in a $1.5-\mathrm{mL}$ microcentrifuge tube, D-mannose $(1.44 \mathrm{mM}, 0.1$ $\mathrm{mL})$ and Con A-FITC $(0.1 \mathrm{~mL})$ were added. The total volume of the final solution was $1.20 \mathrm{~mL}$, where the concentration of D-mannose and Con A-FITC was $120 \quad \mathrm{M}$ and $16 \mathrm{nM}$, respectively. The solutions were shaken for $1 \mathrm{~h}$, which was sufficient for reaching equilibrium as shown in a time-based study, and centrifuged at 12,000 rpm for $30 \mathrm{~min}$ where nanoparticles precipitated to the bottom of the tube. The supernatants were transferred to a quartz cuvette for fluorescence measurements at 480 $\mathrm{nm}$ excitation, and the emissions at $517 \mathrm{~nm}$ were recorded using Felix32 software. The incubation time was determined from a time study where GNPs were incubated with Con A-FITC for varying amount of time. The fluorescence of the supernatant was monitored, and after 1-hour incubation, the intensity no longer changed indicating that the reaction had reached equilibrium. Measurement at each concentration was repeated 5 times, and the mean value of the emission intensities was used for the analysis. For all other GNPs, the same procedure was followed except that the concentrations of the GNPs were varied.

\section{Results and Discussion}

Synthesis of GNPs and ligand density determination.

GNPs were synthesized by coupling carbohydrate ligands to PFPA-functionalized Au NPs. PFPA-thiol compounds of varying spacer lengths (a-d) (Figure 4.2) were synthesized following the procedure shown in Figure 4.1. PFPA-thiols b-d contain 
varying lengths of methylene spacer linkage, whereas PFPA-thiol a has four ethylene oxide (EO) in addition to the 11 methylene units. These PFPA-thiols were used to investigate the impact of spacer linkage on the ligand density and binding affinity of the corresponding GNPs.

Preparation of GNPs followed the photocoupling method developed previously (Figure 4.2). ${ }^{181}$ Citrate-protected $\mathrm{Au}$ NPs were prepared from $\mathrm{HAuCl}_{4}$ and sodium citrate. The size and uniformity were examined by DLS and TEM, which showed the spherically-shaped $\mathrm{Au}$ NPs of $22 \pm 2.6 \mathrm{~nm}$ in diameter. PFPA-thiols were then introduced to the NP surface via a ligand-exchange reaction, and the presence of PFPA on Au NPs was confirmed by ${ }^{1} \mathrm{H}$ NMR and FTIR. Subsequent carbohydrate immobilization was carried out by a photocoupling reaction, and the unattached carbohydrates were removed by membrane dialysis. The resulting GNPs exhibited excellent solubility in water. Furthermore, the centrifuged nanoparticle pellet was easily dissolved in water, and the solution showed no change in the optical property. The GNPs dispersed well in the PBS buffer and the solutions were stable for weeks at $4{ }^{\circ} \mathrm{C}$. 


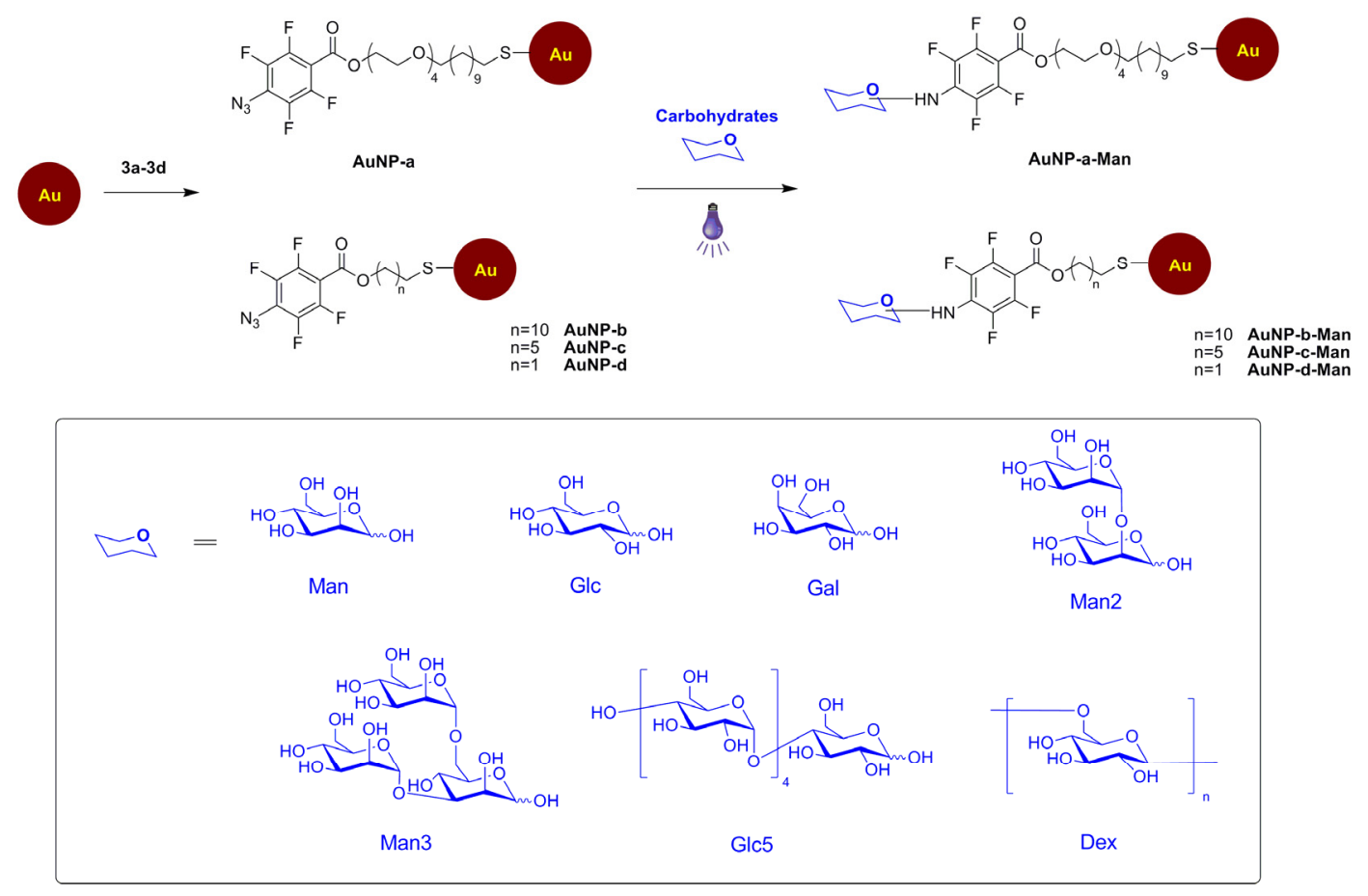

Figure 4.2 Functionalization of Au NPs with PFPA-thiol and subsequent coupling of carbohydrates.

In order to quantitatively analyze GNPs-protein interactions, it is essential to determine the carbohydrate ligand density. The anthrone- $\mathrm{H}_{2} \mathrm{SO}_{4}$ assay, a colorimetric method that was widely used to measure carbohydrate concentrations in solution ${ }^{205}$ and on solid surfaces ${ }^{133}$ was employed to measure the carbohydrate ligand density on the GNPs. ${ }^{181}$ Calibration curves were obtained by treating various concentrations of each carbohydrate with anthrone/sulfuric acid, and the absorption at $620 \mathrm{~nm}$ was plotted against the carbohydrate concentration. Au NPs with immobilized carbohydrate ligands were then subjected to the same assay, and the amount of ligand on the nanoparticles was subsequently derived by comparing 
with the calibration curve. The coupling yield was estimated from the theoretically calculated maximal amount of each ligand that can occupy the 22-nm Au NP assuming a close-packed arrangement of the ligand on the NP. Table 4.1 summarizes the coupling yields of mono-, oligo- and poly-saccharides on Au NPs functionalized with PFPA-thiol a. The results show that the coupling yield increased with the size of the carbohydrate, which was anticipated since the probability of attaching the ligand via $\mathrm{CH}$ insertion reactions increases with the number of available $\mathrm{CH}$ bonds on the ligand.

Table 4.1 Coupling yield of mono-, oligo-, and poly-saccharides on Au NPs.

\begin{tabular}{|c|c|c|c|}
\hline Carbohydrates & $\begin{array}{c}\text { No. of Man/NP } \\
\text { (Theoretical) }^{\mathbf{a}}\end{array}$ & $\begin{array}{c}\text { No of Man/NP } \\
\text { (Experimental) }^{\text {Exp }}\end{array}$ & $\begin{array}{c}\text { Coupling Yield } \\
\text { (\%) }\end{array}$ \\
\hline Man & 6333 & 3991 & $63 \pm 4.2$ \\
\hline Glc & 6333 & 3641 & $57 \pm 5.8$ \\
\hline Man2 & 2303 & 1450 & $67 \pm 6.1$ \\
\hline Man3 & 1974 & 937 & $73 \pm 5.4$ \\
\hline Glc5 & 1299 & 836 & $79 \pm 7.2$ \\
\hline Dex & 24 & 19 & $77 \pm 16$ \\
\hline
\end{tabular}

${ }^{\mathrm{a}}$ Each data was the average of 5 samples.

Detection limit. 
The unique optical property of metal nanoparticles, plasmon resonance absorption, offers a simple and attractive means to study molecular interactions with high sensitivity ${ }^{88,107}$ The binding events occurring at the surface of the Au NPs result in a red shift in the plasmon resonance band, which can be conveniently monitored by UVvis spectroscopy. ${ }^{150}$ To determine the sensitivity of the GNPs in detecting lectins, Dmannose-coupled Au NPs were titrated with Con A, and the UV-vis spectra recorded (Figure 4.3a). The absorbance at $650 \mathrm{~nm}$ vs. Con A concentration was then plotted (Figure 4.3b), and the dynamic linear range of each curve was used to determine the limit of detection (LOD), which was calculated to be $6.2 \mathrm{nM}, 7.4 \mathrm{nM}, 10 \mathrm{nM}$, and 22 nM for AuNP-a-Man, AuNP-b-Man, AuNP-c-Man and AuNP-d-Man, respectively. The results showed that the sensitivity of the GNPs increased with the spacer length, and the lowest LOD was obtained for GNPs prepared from PFPA-thiol a that contains the long and flexible spacer linkage.
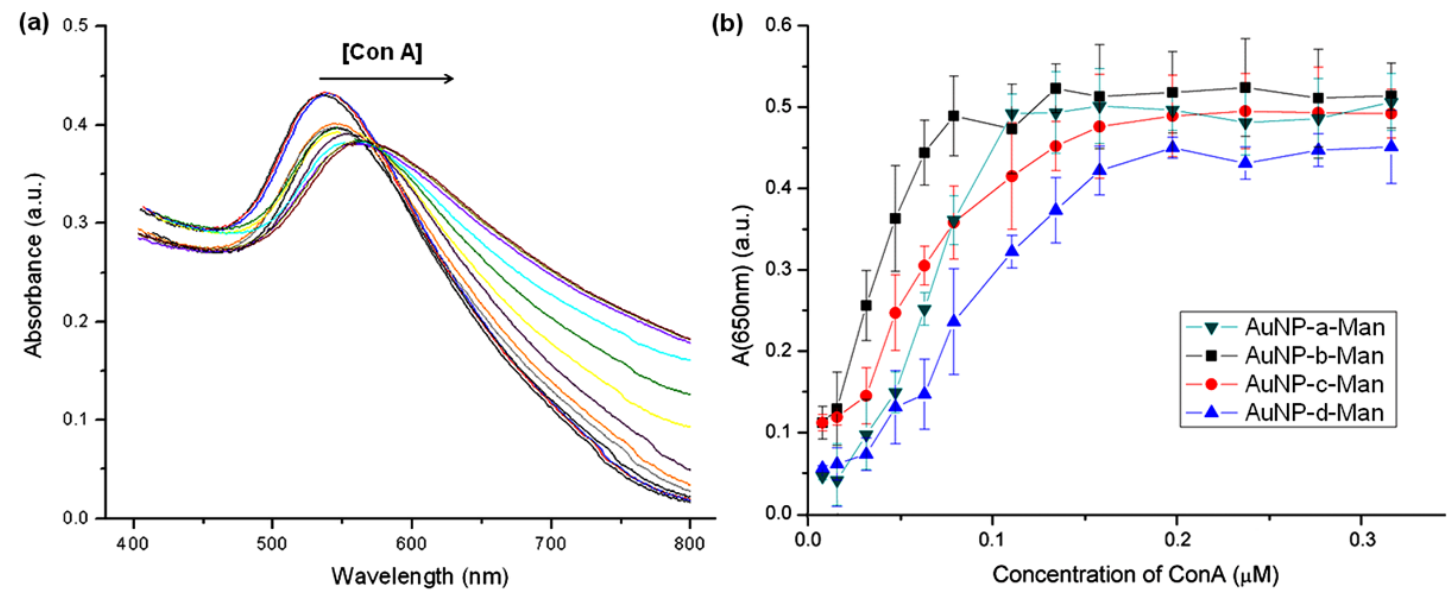

Figure 4.3 (a) UV-vis spectra of AuNP-a-Man upon addition of increasing concentration of Con A. (b) Absorbance of GNPs at $650 \mathrm{~nm}$ vs. Con A concentration. 
Determination of binding affinity by fluorescence competition assay.

We developed a fluorescence competition assay to determine the binding affinity of GNPs with lectins using a fluorescently-labeled lectin and a free competing ligand. In a typical assay, GNPs of varying concentrations and a fixed concentration of a free competing ligand were incubated with Con A-FITC (Figure 4.4a). Two equilibria, Con A with GNPs and Con A with the free ligand, were established in the system (Figure 4.4b). Because a relatively low concentration of Con A was used, no agglomeration was observed in the assay. After the solution was incubated for 1 hour, it was centrifuged, bringing down GNPs including those bound to Con A-FITC. The unbound Con A-FITC and free ligand-Con A conjugate remained in the supernatant, corresponding to the amount of Con A-FITC that did not bind GNPs. The fluorescence of the supernatant was measured (Figure 4.4d), and the intensity at $517 \mathrm{~nm}$ was plotted as a function of the ligand density on GNPs (Figure 4.4e). From this concentration response curve, the $\mathrm{IC}_{50}$ value was determined and the apparent dissociation constant $\mathrm{K}_{\mathrm{d}}$ was computed according to the Cheng-Prusoff equation ${ }^{206}$ (Figure $4.4 \mathrm{c}, \mathrm{K}_{\mathrm{d}}=\mathrm{K}_{\mathrm{d} 2}$ ). 

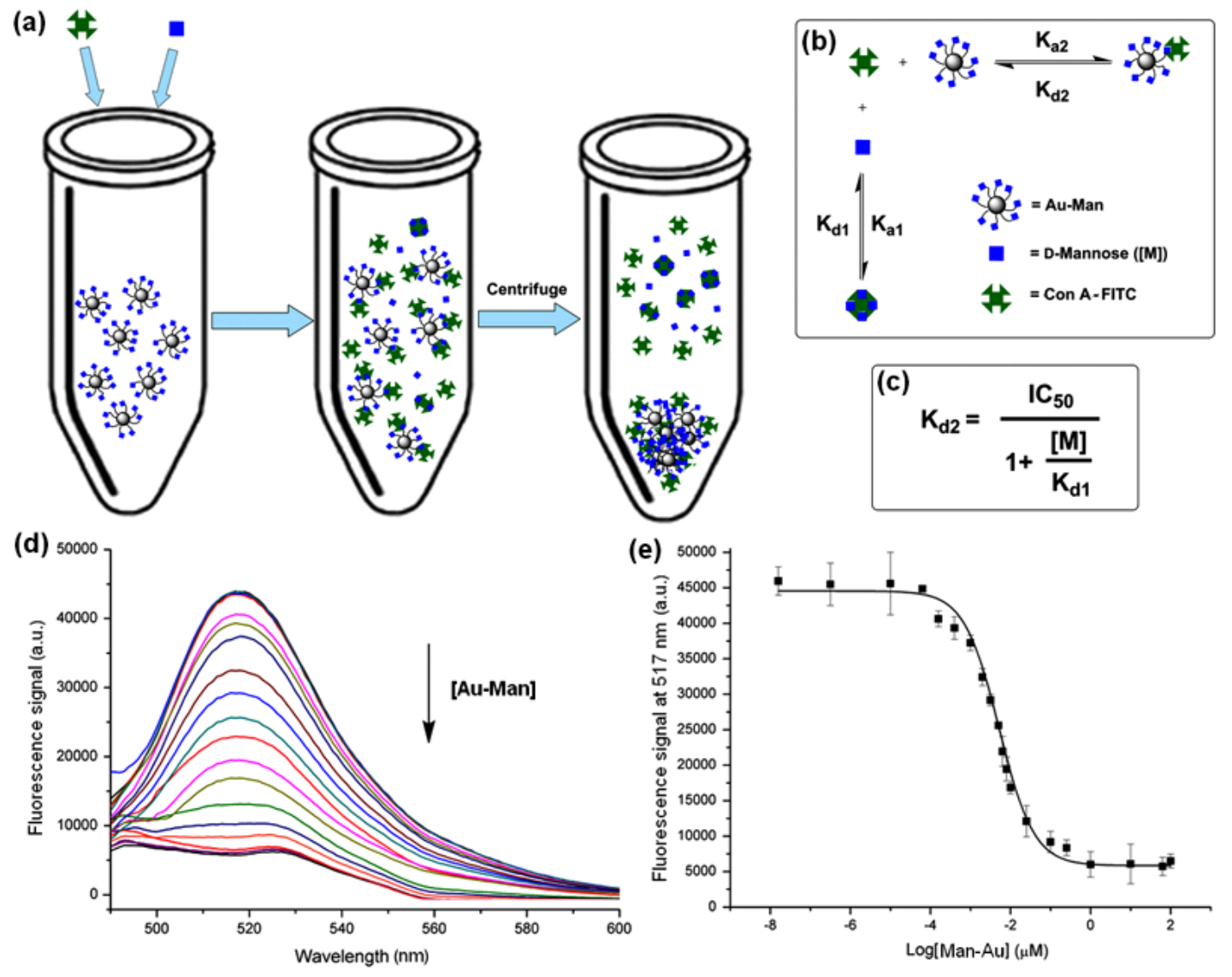

Figure 4.4 (a) The fluorescence-based competition binding assay; (b) two equilibriums established in the system; (c) Cheng-Prusoff equation, where $\mathrm{IC}_{50}=$ concentration of ligand displaying $50 \%$ of specific binding; $[\mathrm{M}]=$ concentration of free ligand, $\mathrm{K}_{\mathrm{d} 1}=$ dissociation constant of the free ligand with Con $\mathrm{A}$; and $\mathrm{K}_{\mathrm{d} 2}=$ dissociation constant of GNPs with Con A; (d) fluorescence spectra of the supernatant as a function of increasing concentration of AuNP-b-Man; (e) concentration response curve, where the $\mathrm{IC}_{50}$ value was obtained.

This method was furthermore validated by several control experiments. First, different concentrations of Con A-FITC, $18 \mathrm{nM}, 40 \mathrm{nM}$ and $80 \mathrm{nM}$, were used in the assay and the binding affinity determined. The results obtained were similar for all three 
concentrations with less than $5 \%$ variation among the three $\mathrm{K}_{\mathrm{d}}$ values. Secondly, different competing ligands, e.g., MeMan, Man2, and Man3, were used in addition to Man. Although the binding affinities of these ligands with Con A were significantly different from that of Man, when they were used as the competing ligand to determine the $K_{d}$ value of the same GNPs, the results were consistent and did not show significant variations. Thirdly, AuNP-a-Man was incubated with Con A-FITC in the absence of the competing ligand. In this case the fluorescence intensity decreased with the concentration of Con A-FITC, and the typical $\mathrm{IC}_{50}$ concentration response curve was not observed. Lastly, Au NPs coupled with Gal, a carbohydrate that does not bind Con A, was tested to ensure that the binding was due to the specific interactions of the surface-bound ligands with the lectin. No significant changes in the fluorescence intensity were observed after varying concentrations of GNPs were incubated with Con A-FITC, demonstrating that the strong affinity of AuNP-a-Man with Con A was indeed due to the specific binding of surface-bound Man with Con A. This result furthermore revealed that the non-specific adsorption of lectin to non-lectin binding carbohydrates was minimal.

With the methodologies developed, we next tested the impact of nanoparticles as the scaffold on the affinity ranking of various carbohydrate ligands with lectins. We chose Man, Man2, and Man3, which have at least several-fold differences in binding affinity between each ligand with Con A (Table 4.2). These ligands were attached to Au NPs using PFPA-thiol 3a, and the apparent $\mathrm{K}_{\mathrm{d}}$ values of the resulting GNPs with Con $\mathrm{A}$ 
were determined by the fluorescence competition assay. An increase in the binding affinity of 4.5-fold and 33-fold was observed for AuNP-a-Man2 and AuNP-a-Man3 in comparison with AuNP-a-Man, respectively. Since the number of ligands on each GNPs was different, when taking into consideration the ligand density, the affinity increase was 12-fold and 141-fold, respectively. This compares well with the affinity ranking of the corresponding free ligands, which was 20 and 158 times higher affinity of Man2 and Man3 than Man with Con A in solution. ${ }^{136,148}$

In addition, the binding affinity of AuNP-a-Glc was determined, and the result showed over 4 orders of magnitude higher affinity than that of the free ligand with Con A (Table 4.2). Compared with the affinity enhancement in the case of Man, however, the binding of AuNP-a-Man with Con A was over 30 times higher than that of AuNP-a-Glc. Considering that the affinity of free Man with Con A was only 4 times higher than Glc, the affinity enhancement when the ligands were attached to NPs was significantly higher. This observation, i.e., the amplification of binding affinity difference due to multivalent interactions, has also been observed for carbohydrate ligands on neoglycopolymers $^{207}$ and dendrimers. ${ }^{208}$ 
Table 4.2 Binding affinity of different GNPs with Con A

\begin{tabular}{|c|c|c|c|}
\hline GNPs & $\begin{array}{c}\text { Number of } \\
\text { ligands/NP }\end{array}$ & $\mathbf{K}_{\mathbf{d}}(\mathbf{n M})$ & $\mathbf{K}_{\mathbf{D}}(\boldsymbol{\mu M})^{\mathbf{a}}$ \\
\hline AuNP-a-Man & 3,991 & $0.43 \pm 0.044$ & $470^{209}$ \\
\hline AuNP-a-Man2 & 1,450 & $0.095 \pm 0.008$ & $24.0^{136}$ \\
\hline AuNP-a-Man3 & 937 & $0.013 \pm 0.002$ & $2.97^{136}$ \\
\hline AuNP-a-Glc & 3,641 & $12.7 \pm 2.5$ & $1,786^{209}$ \\
\hline
\end{tabular}

${ }^{\mathrm{a}} \mathrm{K}_{\mathrm{D}}$ : dissociation constant of the corresponding free ligand with Con $\mathrm{A}$.

\section{Binding affinity with respect to ligand presentation.}

Unlike the free ligand that has the translational and rotational freedom in solution, the surface-bound ligand is no longer an un-restricted entity. Each ligand becomes a member of the nanomaterial carrier and can act cooperatively when interacting with their binding partners. The binding affinity is sensitive to a number of factors including the coupling chemistry, the size of the nanomaterial scaffold, the type and length of the spacer linkage connecting the ligand and the nanomaterial, the flexibility/rigidity of the spacer, the density of ligands and the distance between them. In the present study, the ligand density, linker length, and nanoparticle size were varied, and their impacts on the binding affinities of the resulting GNPs were investigated. 
Ligand density. To control the surface ligand density, we employed the mixed SAM approach where a non-photoactive thiol together with PFPA-thiol was used to functionalize Au NPs. Solutions containing PFPA-thiol $\mathbf{a}$ or $\mathbf{b}$ and 1-hexanethiol at varying mole ratios were used to treat Au NPs. Man was subsequently coupled and the density of attached ligand measured (Table 4.3). The apparent $K_{d}$ value of the resulting GNPs was then determined using the fluorescence competition assay described above. Results showed that the binding affinity generally increased with the ligand density. For AuNP-a-Man, however, there seems to be a maximal affinity for Au NPs treated with 98\% PFPA-thiol a at the ligand density of 3,004 Man/NP. This maximal affinity was not observed in the case of AuNP-b-Man, likely due to the lower ligand density even at $100 \%$ PFPA-thiol treatment (2,824 Man/NP). To investigate the generality of this observation, Man2 and Man3 were coupled on Au NPs treated with mixed SAM of 1-hexanethiol and PFPA-thiol a, and the $K_{d}$ values were measured with respect to the ligand density. The highest binding affinity of the resulting GNPs occurred at $50 \%$ and $30 \%$ of PFPA-thiol a in the mixed SAM, corresponding to the ligand density of $289 \mathrm{Man} 2 / \mathrm{NP}$ and $132 \mathrm{Man} 3 / \mathrm{NP}$, respectively (Figure 4.5). 
Table 4.3 Binding affinity as a function of ligand density.

\begin{tabular}{|c|c|c|c|c|}
\hline \multirow{2}{*}{$\begin{array}{c}\text { PFPA- } \\
\text { thiol } \\
(\mathbf{m o l e} \%)\end{array}$} & $\begin{array}{c}\text { Number of } \\
\text { Man/NP }\end{array}$ & $\mathbf{K}_{\mathbf{d}}(\mathbf{n M})^{\mathbf{a}}$ & $\begin{array}{c}\text { Number of } \\
\text { Man/NP }\end{array}$ & $\mathbf{K}_{\mathbf{d}}(\mathbf{n M})^{\mathbf{a}}$ \\
\hline $10 \%$ & 107 & $27.4 \pm 2.3$ & 99 & $92 \pm 12$ \\
\hline $30 \%$ & 283 & $8.67 \pm 0.87$ & 260 & $16.1 \pm 5.5$ \\
\hline $50 \%$ & 544 & $1.93 \pm 0.25$ & 549 & $12.3 \pm 4.1$ \\
\hline $70 \%$ & 1,444 & $1.33 \pm 0.31$ & 1,196 & $10.8 \pm 1.8$ \\
\hline $90 \%$ & 2,275 & $1.01 \pm 0.22$ & 1,726 & $9.6 \pm 3.0$ \\
\hline $95 \%$ & 2,756 & $0.88 \pm 0.14$ & 2,090 & $7.1 \pm 1.7$ \\
\hline $98 \%$ & 3,004 & $0.39 \pm 0.09$ & 2,474 & $5.0 \pm 1.0$ \\
\hline $100 \%$ & 3,991 & $0.43 \pm 0.044$ & 2,824 & $4.0 \pm 0.7$ \\
\hline
\end{tabular}

${ }^{a}$ Each data was the average of 3 measurements each on 5 samples. 

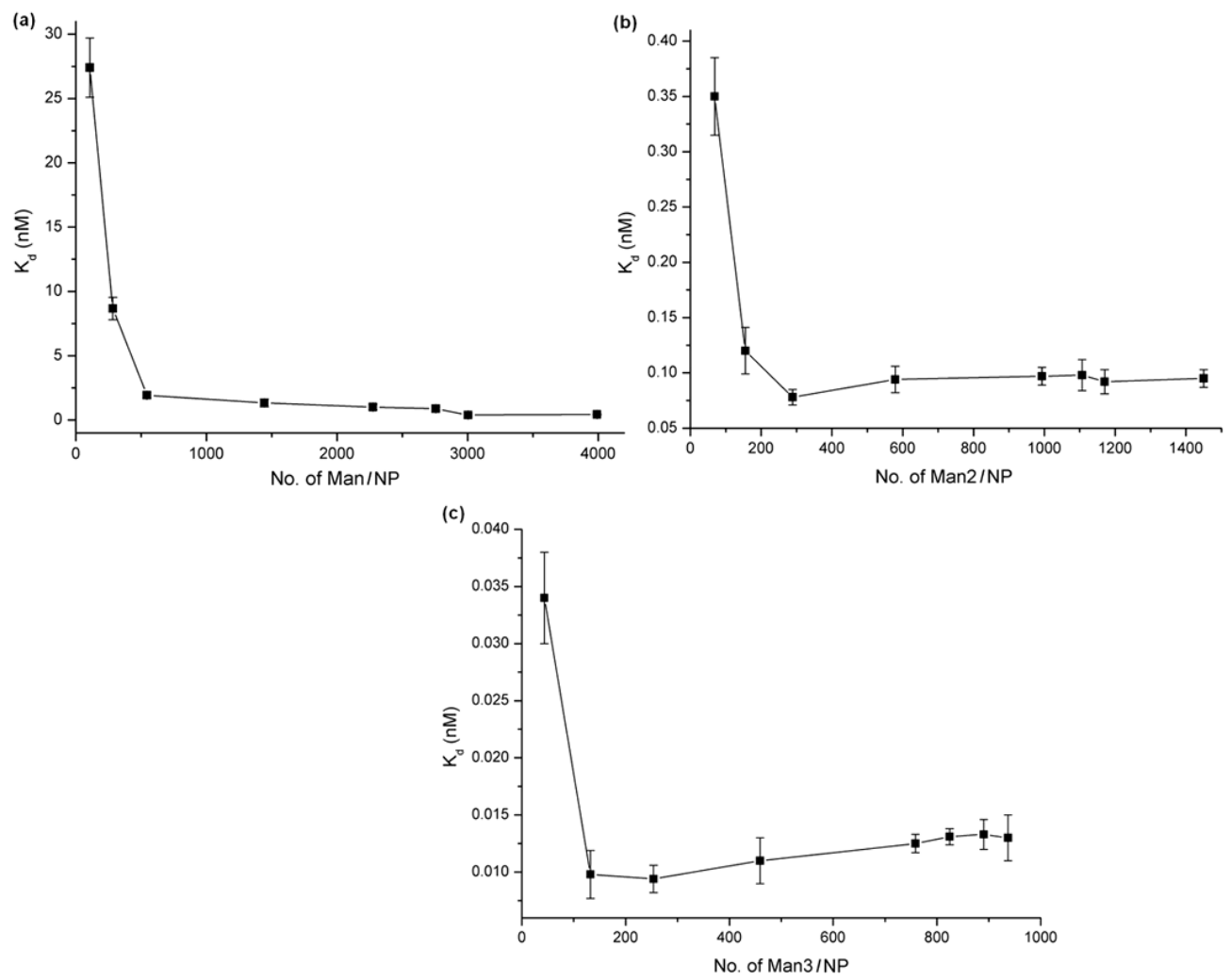

Figure 4.5. Binding affinity vs. ligand density for Man (a), Man2 (b), and Man3 (c) with Con A. Each data point was the average of 3 measurements each on 5 samples.

A few general observations can be drawn from the ligand density studies. For all three ligands, there was a sudden increase in binding affinity, and after which, the $\mathrm{K}_{\mathrm{d}}$ values remained more or less constant. The ligand density at which the drastic change in binding affinity occurred was estimated from the intercept of the two relatively linear curves in each graph, which was 370 Man/NP, 166 Man2/NP and 138 Man3/NP, respectively. Assuming that the ligands were evenly distributed on the GNPs, the footprint occupied by each ligand on the nanoparticle surface was calculated to be 4.1 $\mathrm{nm}^{2}, 9.2 \mathrm{~nm}^{2}$ and $11.0 \mathrm{~nm}^{2}$, and the distance between neighboring ligands were 
approximately $2.0 \mathrm{~nm}, 3.0 \mathrm{~nm}$ and $3.3 \mathrm{~nm}$ for Man, Man2 and Man3, respectively (see detailed calculation in Supporting Information). Note that the distance between each binding site on Con $\mathrm{A}$ is $6.5 \mathrm{~nm}$ as determined by X-ray structural analysis. ${ }^{183}$ This distance is larger than the ligand spacing on NPs indicating that the binding is not monovalent and the higher ligand density is necessary for the enhanced affinity. The internal diffusion mechanism, which states that a lectin molecule "binds and jumps" from ligand to ligand along the scaffold, ${ }^{190}$ can be applied to explain the results. According to the mechanism, the more ligands there are on the scaffold, the longer the dwelling time of the lectin on the ligands, and the slower the lectin would dissociate. This affinity increase was observed for all three ligands where the $K_{d}$ values decreased drastically at lower ligand density (Figure 4.5). The affinity started to decrease after the maximal affinity was reached, and this decrease in affinity was more pronounced as the size of the ligand increased. This was likely due to the steric effect where the larger ligand hinders the lectin binding. ${ }^{210}$

Spacer. PFPA-thiols a, b, $\mathbf{c}$ and $\mathbf{d}$ were used as the coupling agent to evaluate the effect of spacer linker on the binding affinity of the resulting GNPs. PFPA-thiols $\mathbf{c}, \mathbf{d}$ contain a shorter spacer of six and two methylene units, and the $\mathrm{K}_{\mathrm{d}}$ values of the corresponding GNPs were $15 \mathrm{nM}$ and $19 \mathrm{nM}$, respectively (Table 4.4). When Au NPs were functionalized with PFPA-thiol b having 11 methylene units, $K_{d}$ decreased to 4.0 $\mathrm{nM}$, which represents 4-5 times increase in binding affinity as compared to the shorter spacer $\mathbf{c}$ and d. When PFPA-thiol a was used, the binding affinity increased an 
additional order of magnitude in comparison to PFPA-thiol b. Because the ligand density increases with the spacer length, it could also contribute to the observed enhancement in the binding affinity. However, the density increase alone could not account for the magnitude of the affinity enhancement. At the similar ligand density, for example, 544 and 549 Man/NP for AuNP-a-Man and AuNP-b-Man, respectively, the binding affinity was 6 times higher for AuNP-a-Man than AuNP-b-Man (Table 4.4). This affinity increase was therefore solely caused by the difference in the spacer linkage between the two GNPs. In order to quantitatively assess the binding affinity enhancement of different GNPs, an affinity enhancement factor (EF) was used, as defined in Eq. 4.1,

$$
\mathrm{EF}=\mathrm{K}_{\mathrm{D}} /\left[\mathrm{K}_{\mathrm{d}} \mathrm{x}\right]
$$

where $K_{D}$ is the dissociation constant of free ligand with Con $A$, which is $470 \mu \mathrm{M}$ for Man, ${ }^{209} \mathrm{~K}_{\mathrm{d}}$ is the apparent dissociation constant of GNPs with Con A, and $\mathrm{N}$ is the number of ligands on each GNP. Here, the ligand density is taken into consideration, and the EF value can therefore be used to rank binding affinity with respect to the difference in the spacer linkage. Results in Table 4.4 show that the EF values for AuNP-d-Man and AuNP-c-Man were similar, 16, whereas that of AuNP-b-Man was 2.6 times higher at 42, and that of AuNP-a-Man was in addition 17 times higher at 274 . These results clearly demonstrate that longer spacers led to enhanced binding affinity of GNPs with lectins. The spacer elevates the ligand further from the solid substrates. On curved surfaces such as nanoparticles, a longer spacer would also result in a larger distance between the ligands at the same ligand density. Both events would 
Table 4.4 Binding affinity of GNPs with Con A.

\begin{tabular}{|c|c|c|c|}
\hline GNPs & Number of Man/NP & $\mathbf{K}_{\mathbf{d}}(\mathbf{n M})^{\mathbf{a}}$ & EF \\
\hline AuNP-a-Man & 3,991 & $0.43 \pm 0.044$ & 274 \\
\hline AuNP-b-Man & 2,824 & $4.0 \pm 0.72$ & 42 \\
\hline AuNP-c-Man & 1,959 & $15 \pm 2.0$ & 16 \\
\hline AuNP-d-Man & 1,590 & $19 \pm 2.2$ & 16 \\
\hline
\end{tabular}

${ }^{a}$ Each data was the average of 5 samples, 3 measurements each.

reduce the steric hindrance when the lectin approaches the ligands, making the ligands more accessible for interacting with the lectin.

Nanoparticle size. It is well-established that the physical and chemical absorption, and the catalytic property of gold nanoparticles are highly size-dependent. ${ }^{171-173}$ In this study, the effect of nanoparticle size on binding affinity was investigated by varying the diameter of Au NPs. In addition to the 22-nm nanoparticles, Au NPs of $7 \mathrm{~nm}, 14$ $\mathrm{nm}$, and $30 \mathrm{~nm}$ in diameter were synthesized using the same protocol (Figures 4.6), and Man was subsequently conjugated using PFPA-thiol b. The ligand densities of the resulting GNPs and their binding affinities were measured (Table 4.5). As expected, the number of ligands attached on Au NPs increased with the size of the nanoparticles. The binding affinity of GNPs with particle sizes of $7 \mathrm{~nm}, 14 \mathrm{~nm}$, and $22 \mathrm{~nm}$ were similar, whereas a decrease of about 6-fold in binding affinity was observed for the 
30-nm GNPs. The EF values calculated for each GNPs showed a considerable sizedependent effect, with the EF value increasing with decreasing particle size. Smaller nanoparticles yielded the highest affinity enhancement, likely due to their large surface-to-volume ratio and higher mobility in solution.
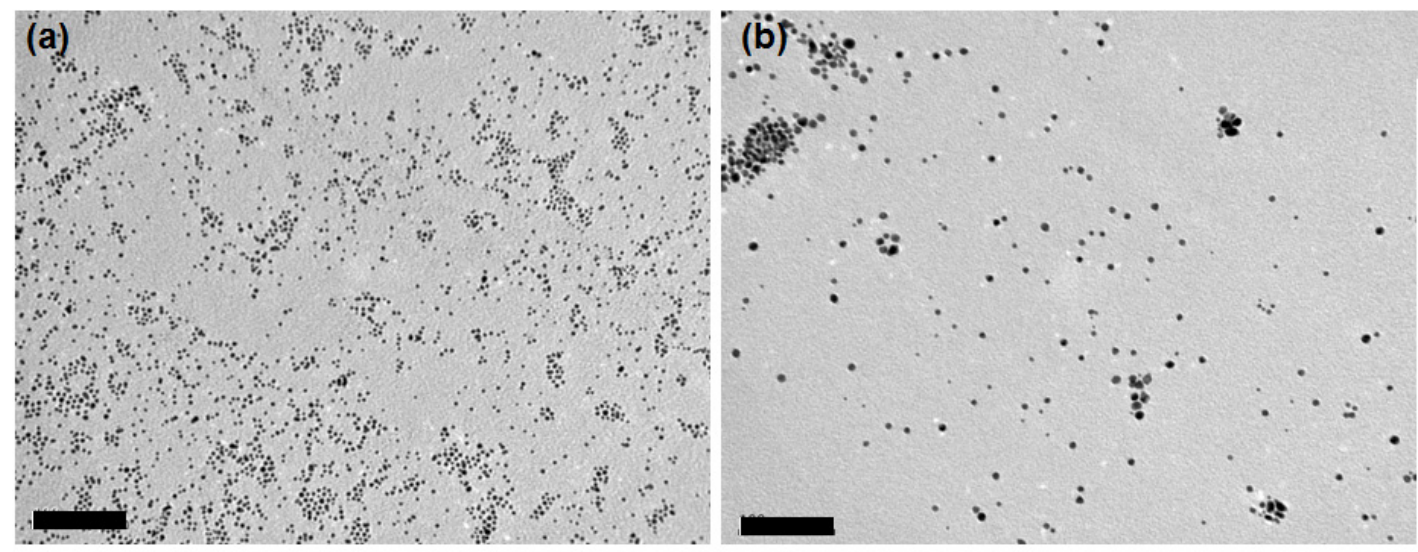

(c)
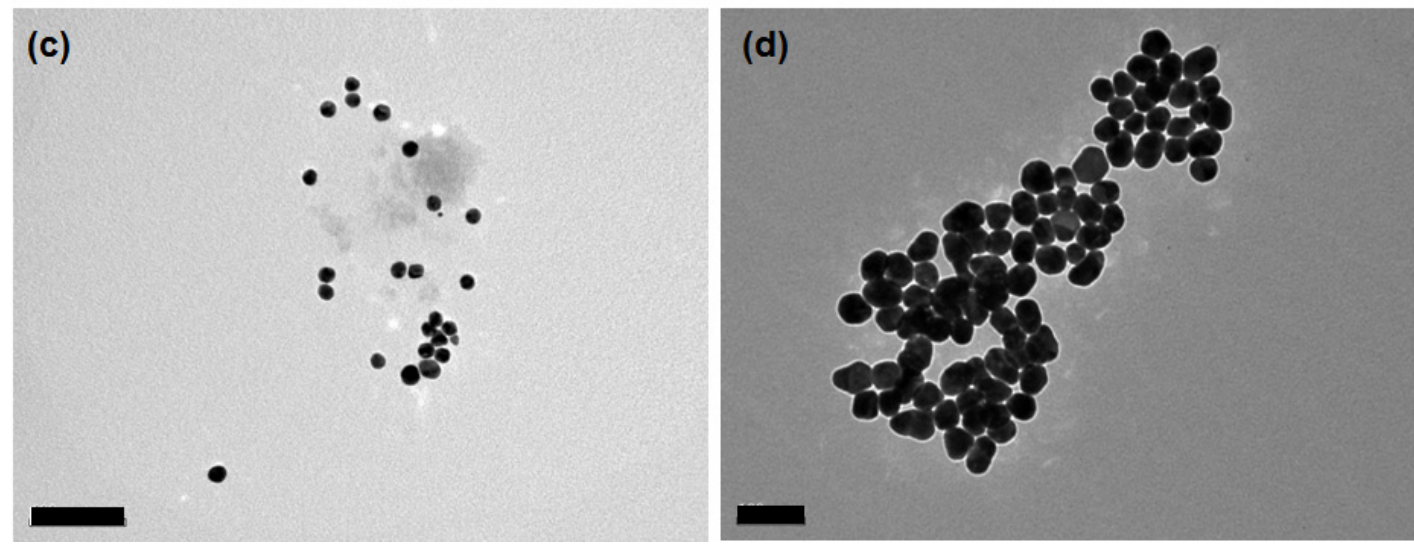

Figure 4.6 TEM images of gold nanoparticles in 4 different sizes: $7 \mathrm{~nm}$ (a), $14 \mathrm{~nm}$ (b), $22 \mathrm{~nm}$ (c) and $30 \mathrm{~nm}(\mathrm{~d})$. 
Table 4.5 Binding affinity of GNPs of varying sizes

\begin{tabular}{|c|c|c|c|}
\hline $\begin{array}{c}\text { Au NP Diameter } \\
(\mathbf{n m})\end{array}$ & Number of Man/NP & $\mathbf{K}_{\mathbf{d}}(\mathbf{n M})$ & $\mathbf{E F}$ \\
\hline $7.2 \pm 1.8$ & 297 & $3.38 \pm 0.67$ & 468 \\
\hline $14 \pm 2.6$ & 1,127 & $3.14 \pm 0.49$ & 132 \\
\hline $22 \pm 3.3$ & 2,824 & $3.99 \pm 0.81$ & 42 \\
\hline $30 \pm 4.0$ & 4,486 & $24.8 \pm 3.1$ & 4.2 \\
\hline
\end{tabular}

\section{Conclusion}

In conclusion, we developed a fluorescence-based competition assay to determine the apparent dissociation constants of GNPs with lectin. The assay was successfully used to determine the $\mathrm{K}_{\mathrm{d}}$ values and to evaluate the binding affinity of GNPs. When carbohydrate ligands were attached on the Au NP scaffold, their interactions with lectins were drastically enhanced, and several orders of magnitude increases in binding affinity were observed between GNPs and lectin. Systematic studies were conducted to investigate the impacts of nanoparticle size, spacer length, ligand size and density on the binding affinity of GNPs. Results show that the lectin binding to ligands on GNPs is profoundly affected by how the ligands are displayed on the NP surface. Findings from this study are important that GNPs with controlled binding affinity can be readily synthesized by varying ligand density, spacer linker, and scaffold configuration. This will open up immense opportunities for tailor-made glyconanomaterials where the ligand display can be exploited to tune their bioaffinities. 


\section{Supporting Information}

Calculations of ligand density and distance
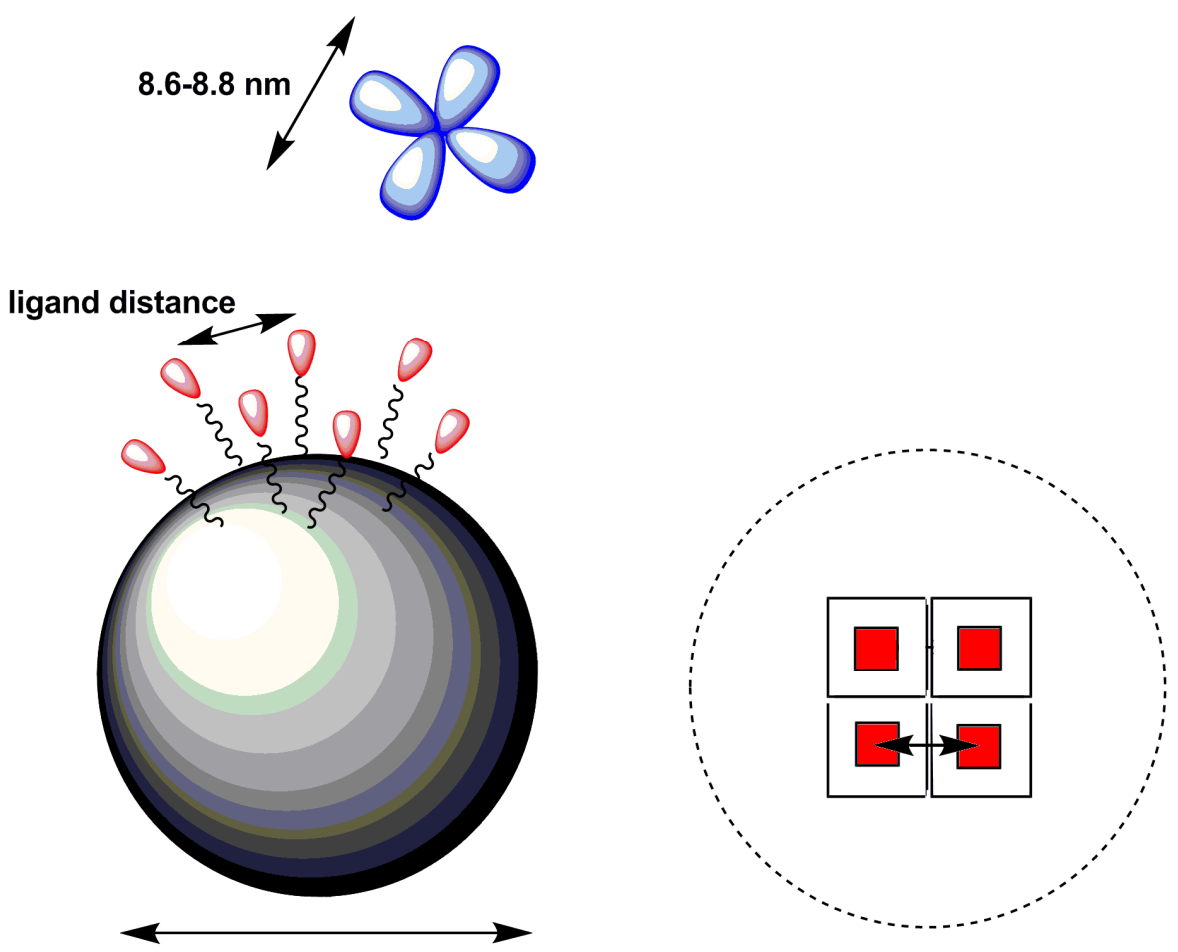

$22 \mathrm{~nm}$

Figure 4.7 Schematic illustration of Con A and GNP.

Assuming that carbohydrate occupies in space by taking the shape of a square, the ligand molecule is then projected to the surface using Chem 3D (CambridgeSoft, Ultra, version 9.0), and the surface area of each Man, Man2 and Man3 molecule were calculated to be $0.24,0.66$, and $0.77 \mathrm{~nm}^{2}$, respectively.

The ligand-ligand distance $(L)$ can be calculated from the following:

$\mathrm{L}=\sqrt{\frac{\mathrm{S}_{\mathrm{NP}}}{\mathrm{N}}}$ 
where $S_{N P}$ is the surface area of $A u N P$, and $N$ is the number of ligands attached. At the point where there was a sudden increase in binding affinity, the number of ligands was determined from the intercept of the two relatively linear curves of each graph which was 370, 166 and 138 ligands per NP for Man, Man2, and Man3, respectively. Therefore, the ligand distance at the deflection point is 2.0, 3.0 and $3.3 \mathrm{~nm}$ for Man, Man2 and Man3, respectively. 


\section{Chapter 5. Applications of Glyconanomaterials in Bioanalysis}

The work in Section 5.1 was published on "Wang, X.; Matei, E.; Deng, L. Q.;

Ramström, O.; Gronenborn, A. M.; Yan, M., Multivalent Glyconanoparticles with Enhanced Affinity to Anti-Viral Lectin Cyanovirin-N Chem. Commun. 2011, 47, 8620-8622"

http://pubs.rsc.org/en/Content/ArticleLanding/2011/CC/C1CC12981C

Reproduced by permission of The Royal Society of Chemistry

The work in Section 5.2 was published on "Wang, X.; Ramström, O.; Yan, M., Dyedoped silica nanoparticles as efficient labels for glycans. Chem. Commun. 2011, 47 (14), 4261-4263" (Section 3.2)

http://pubs.rsc.org/en/Content/ArticleLanding/2011/CC/c0cc05299j

Reproduced by permission of The Royal Society of Chemistry

The work in Section 5.3 was submitted for publication. 


\subsection{Signal Enhancement Study Glycan-lectin Interactions using Anti-HIV Lectins}

In this chapter, a specific platform was presented for the binding signal enhancement of glyconanoparticle with biologically important anti-HIV lectin Cyanovirin-N in a rigorous and coherent way. Our method enables sensitive recognition of different type of lectin mutants with various binding sites using high-mannose conjugated gold nanoparticles, and more significantly visualize the difference using UV-vis and TEM techniques. No other known methods can efficiently accomplish this. This fact alone results in a platform, where the effect of ligand display on multivalency can be efficiently monitored, as well as quantitatively analyzed. The present coupling method is novel, using only unprotected high mannose oligosaccharides structures. We have demonstrated that our method is general, versatile, and has been validated with other solution- and surface-based assays. It is of high importance that our results have shown that the glyconanoparticles system is highly valid in distinguishing multivalency effects based on nanoparticle presentation. The binding affinities of both Man2 and Man3-conjugated nanoparticles were found several orders of magnitude higher than those of free Man2 and Man3 in solution, based on ITC results.

Lectins, carbohydrate-binding proteins, play critical roles in a plethora of biological processes. ${ }^{[1]}$ An in-depth understanding of carbohydrate-lectin interactions is not only fundamentally important for elucidating their biological functions, but also of 
outstanding practical value in the design and development of therapeutics and diagnostic tools. Cyanovirin- $\mathrm{N}$ is an $11 \mathrm{kDa}$ cyanobacterial lectin that exhibits inhibitory activity against a number of viruses, including HIV, at concentrations as low as nanomolar. Its anti-HIV activity is mediated by binding to the high-mannose (HM) structures on the envelope glycoprotein gp 120. ${ }^{[2]}$ Previous studies established that the binding epitope(s) on $N$-linked high oligomannosides for CV-N comprised $\alpha$ D-Man $p-(1 \rightarrow 2)-\alpha-\mathrm{D}-\mathrm{Man} p \quad$ moieties on the glycan's D1 and D3 arms. ${ }^{[3]}$ Multivalency, resulting in cooperative interactions of multiple ligands with multiple receptors, is a general phenomenon that occurs in many biological processes involving molecular recognition. Multivalent interactions are often significantly stronger than the corresponding monovalent interactions, and, as such, the design and creation of multivalent reagents is an important strategy for generating diagnostic and therapeutic tools. ${ }^{[4]}$ In glycobiology, these kinds of approaches are especially relevant given the commonly observed weak affinities between glycans and lectins. ${ }^{[5]}$ On the other hand, high glycan structures exhibit drastically enhanced apparent affinities, compared to the monovalent ligands. However, the synthesis of high glycans is tremendously demanding, involving multiple protection/ deprotection steps and complex stereochemistry control. As such, their availability is limited. An alternative approach for creating multivalency is to use a scaffold, such as polymers, lipids or nanomaterials, on which multiple copies of a ligand can be presented, thereby generating a multivalent ligand. ${ }^{[6]}$ For example, Melander and coworkers prepared small molecule-coated gold nanoparticles (AuNPs) as effective inhibitors for HIV 
fusion, ${ }^{[7]}$ and Gervay-Hague's group reported that galactosyl- and glucosylfunctionalized AuNPs exhibited 300 times better binding to gp $120 .{ }^{[8]}$ In previous studies from our group, we showed that carbohydrate ligands conjugated to AuNPs exhibited affinities up to five orders of magnitude higher than those of the corresponding monomeric ligands with lectins. ${ }^{[9]}$

Here, we conjugated two low-mannoses, Man2 and Man3, to the AuNP scaffold, and investigated the binding affinity of the resulting GNPs with CV-N lectins (Figure 5.1). In order to derive quantitative numbers for the affinity enhancement caused by AuNPs, we developed a fluorescence competition assay and determined the apparent dissociation constant of GNP binding to CV-N $\left(\mathrm{K}_{\mathrm{D}}\right)$. The results from this assay were compared with the $\mathrm{K}_{\mathrm{d}}$ values of monomeric glycan binding to CV-N using isothermal titration calorimetry (ITC).

\section{Experimental Section}

\section{Preparation of gold glyconanoparticles}

AuNPs, $22 \mathrm{~nm}$ in diameter, were prepared and functionalized with PFPA-disulfide following the same procedure as described in a previous chapter. ${ }^{[9]}$ The subsequent carbohydrate coupling was carried out photochemically as reported previously. ${ }^{[10]}$. Before the binding experiments, the GNPs were incubated in $20 \mathrm{mM}$ sodium phosphate buffer solution (pH 6.0), containing $0.01 \% \mathrm{NaN}_{3}, 0.01 \%$ Tween 20 and 3\% BSA for 1 hour, centrifuged, and placed in buffer without BSA until further use. 
CVN binding assays

GNP-M3 $(5 \mathrm{nM}, 1.0 \mathrm{~mL})$ was incubated in a solution of $\mathrm{CVN}^{\mathrm{Q} 50 \mathrm{C}}$ or $\mathrm{CVN}^{\mathrm{MutDB}}$ in 20 $\mathrm{mM}$ sodium phosphate buffer solution $(10 \mu \mathrm{M}, 1.0 \mathrm{~mL}), \mathrm{pH}$ 6.0, containing $0.01 \%$ $\mathrm{NaN}_{3}$ for 1 hour with constant shaking. UV-vis spectra of the resulting solutions were recorded on a Perkin Elmer Lambda 45 UV-vis spectrophotometer, and each measurement was performed at least 3 times.

Isothermal Titration Calorimetry (ITC)

ITC experiments of Man3 binding to CVN were performed using an ITC200 Microcalorimeter from Microcal, LLC. (Northampton, MA) in $50 \mathrm{mM}$ sodium phosphate buffer, $\mathrm{pH} 7.5,200 \mathrm{mM} \mathrm{NaCl}, 0.02 \% \mathrm{NaN}_{3}$. The concentration of $\mathrm{CV}-\mathrm{N}$ was $50 \mu \mathrm{M}$, and that of Man3 was $0.64 \mathrm{mM}$. In each individual experiment, $\sim 38 \mu \mathrm{L}$ of Man3 was injected through the computer-controlled $40-\mu \mathrm{L}$ micro-syringe at an interval of 4 min into the protein solution in the same buffer $($ cell volume $=200 \mu \mathrm{L})$ while stirring at $350 \mathrm{rpm}$. Calorimetric titrations of Man2 binding to CVN were performed using a VP-ITC isothermal titration calorimeter (MicroCal, LLC; Northampton, MA). Titrations were carried out at $30^{\circ} \mathrm{C}$ in the same buffer as described above for Man3. A $35 \mu \mathrm{M} \mathrm{CV-N}$ solution was placed in the calorimeter cell $(\sim 1.44$ $\mathrm{mL}$ active volume), stirred at $310 \mathrm{rpm}$, and 9- $\mu \mathrm{L}$ aliquots of $1.5 \mathrm{mM}$ Man2 solution were added at 2 min intervals from a $295-\mu \mathrm{L}$ stirring syringe. A total of 30 injections 
were performed. The experimental data were fitted to a theoretical titration curve using the software supplied by MicroCal. A standard two-site model was used with $\Delta \mathrm{H}$ (enthalpy change, in $\mathrm{kcal} / \mathrm{mol}$ ), $\mathrm{K}_{\mathrm{a}}$ (association constant, in $\mathrm{M}^{-1}$ ), and $\mathrm{N}$ (number of binding sites) as the variables.

\section{Fluorescence competition binding assays}

The previously reported protocol was adapted, ${ }^{[9]}$ as described below. A series of GNPM2 solutions were prepared by diluting a stock solution $(10 \mathrm{nM})$ to concentrations between $5 \mathrm{nM}$ and $1 \times 10^{-8} \mathrm{nM}$. A stock solution of Cy5-CVN $(1.20 \mu \mathrm{M})$ was prepared in $\mathrm{pH} 6.0$ sodium phosphate buffer $(20 \mathrm{mM})$. To a solution of GNP-M2 $(1 \mathrm{~mL})$ in a 1.5-mL microcentrifuge tube, Man2 (0.48 mM, $0.1 \mathrm{~mL})$ and Cy5-CVN $(1.2 \mu \mathrm{M}, 0.1$ $\mathrm{mL}$ ) were added. The total volume of the final solution was $1.20 \mathrm{~mL}$, and the concentrations of Man2 and Cy5-CVN were $40 \mu \mathrm{M}$ and $100 \mathrm{nM}$, respectively. The solutions were shaken for 1 hour, and then centrifuged at 12,000 rpm for 30 min until all nanoparticles were completely pelleted at the bottom of the tube. The supernatants were taken out for fluorescence measurement using a PTI spectrofluorimeter (Photon Technology International). Excitation was at $649 \mathrm{~nm}$ and emission was recorded at $666 \mathrm{~nm}$ for analysis. The same procedure was followed for GNP-M3, except that Man3 was used as the competing ligand. Measurement for each concentration was repeated 5 times, and values were averaged. 
$\mathrm{K}_{\mathrm{D} 1}$ and $\mathrm{K}_{\mathrm{D} 2}$, apparent dissociation constants for GNP binding to the glycan-binding site on Domain A and Domain $\mathrm{B}$ of $\mathrm{CVN}^{\mathrm{Q} 50 \mathrm{C}}$, respectively, were obtained from bestfitting the response curves, using a two-site competitive binding model with the equation in KaleidaGraph software.

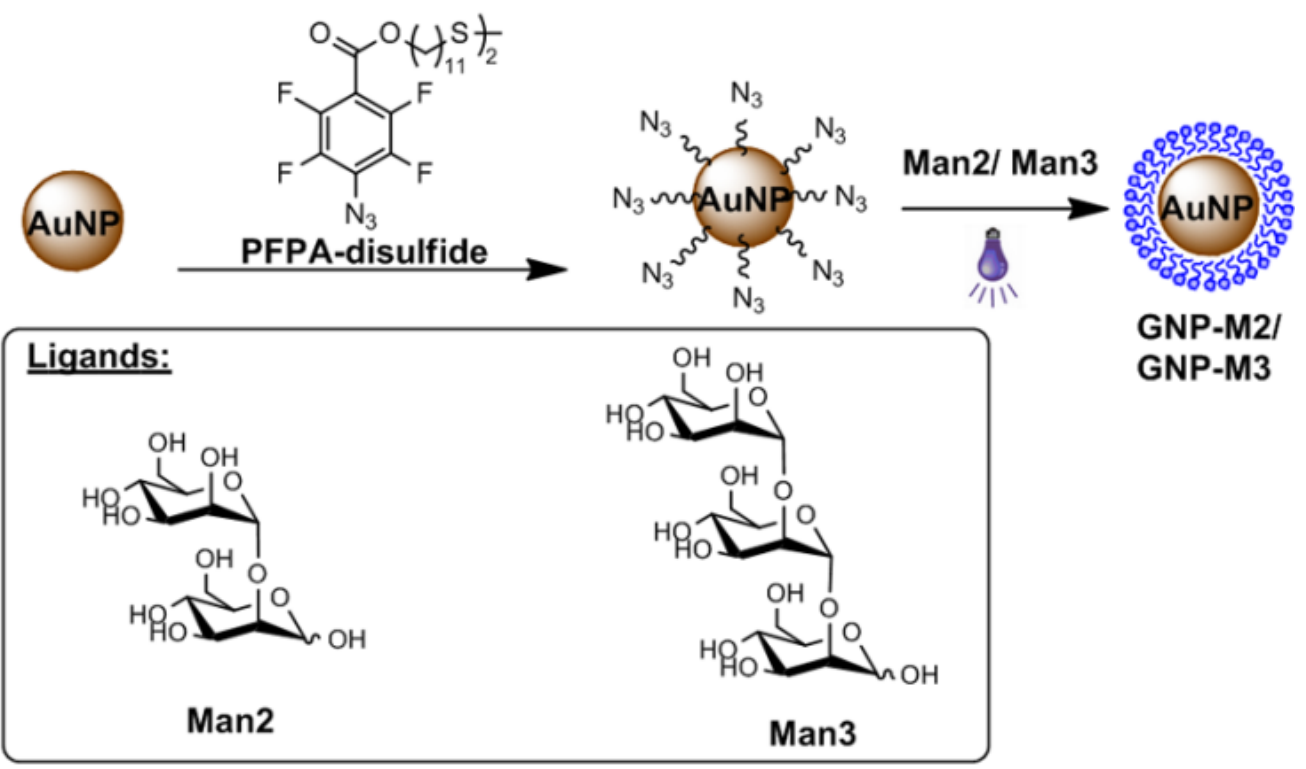

Figure 5.1 Synthesis of Man2- and Man3-conjugated AuNPs GNP-M2 and GNP-M3.

\section{Results and discussion}

GNP-M2 and GNP-M3 were prepared following a previously established procedure ${ }^{[10]}$ outlined in Figure 5.1. Uniform, $\sim 22 \mathrm{~nm}$ AuNPs were synthesized by the Turkevich method ${ }^{[11]}$ and were subsequently functionalized with PFPA-disulfide (Figure 5.1). Man2 and Man3 were then conjugated to the PFPA-functionalized AuNPs using a photocoupling method ${ }^{[10]}$ by way of a $\mathrm{CH}$ insertion reaction of the photogenerated perfluorophenylnitrene. ${ }^{[12]}$ The ligand density was determined using a 
colorimetric approach with anthrone/sulfuric acid. Values of 1,516 \pm 232 Man2 and 1,037 \pm 148 Man3 were obtained for GNP-M2 and GNP-M3, respectively. Binding affinities of GNP-M3 to CV-N was evaluated using two CV-N variants: $\mathrm{CVN}^{\mathrm{MutDB}}$ and $\mathrm{CVN}^{\mathrm{Q} 50 \mathrm{C}} \cdot \mathrm{CVN}^{\mathrm{Q} 50 \mathrm{C}}$ is essentially a wild-type variant, comprising two separate glycan binding sites one on Domain A and one on Domain B. ${ }^{[3 a, 3 c]}$ Domain A exhibits a slight preference for the Man3 units and domain B for the Man2 units. ${ }^{[13,3 c]}$ The Cys substitution at position 50 was introduced to allow for specific fluorescence labeling of CV-N without interfering with glycan binding. In the $\mathrm{CVN}^{\mathrm{MutDB}}$ variant on the other hand, the glycan binding site on domain B is completely eliminated, while the site on domain A still can bind glycan ligands. Since this variant no longer can cross-link glycans on gp120, it has lost its anti-HIV activity. ${ }^{[14]}$ Therefore, in interactions with GNPs, we would expect that $\mathrm{CVN}^{\mathrm{Q50C}}$ can act as a crosslinker and form a complex with GNP-M3, while no such crosslinking should be possible between GNP-M3 and CVN ${ }^{\mathrm{MutDB}}$. Indeed, GNP-M3 treatment with $\mathrm{CVN}^{\mathrm{Q} 50 \mathrm{C}}$ caused a red shift from $529 \mathrm{~nm}$ to $542 \mathrm{~nm}$ in the surface plasmon resonance (SPR) band of AuNPs (Figure 5.2a), indicative of particle size growth. ${ }^{[15]}$ Such an increase in particle size was further confirmed by TEM which revealed the presence of clusters of aggregated particles (Figure 5.2c). When GNP-M3 was treated with CVN ${ }^{\mathrm{MutDB}}$, however, no SPR shift was observed (Figure 5.2b). TEM images were devoid of aggregates and only isolated single particles were observed in this case (Figure 5.2c). Dynamic light scattering (DLS) measurements of CV-N treated GNP-M3 particles yielded average particle sizes of $25.9 \pm 3.5 \mathrm{~nm}$ and $38.3 \pm 4.6 \mathrm{~nm}$ for $\mathrm{CVN}^{\mathrm{MutDB}}$ and 
$\mathrm{CVN}^{\mathrm{Q} 50 \mathrm{C}}$, respectively. These results are all consistent with our previous structural studies on $\mathrm{CVN}^{\mathrm{MutDB}}$ that revealed a single glycan binding site. ${ }^{[14]}$
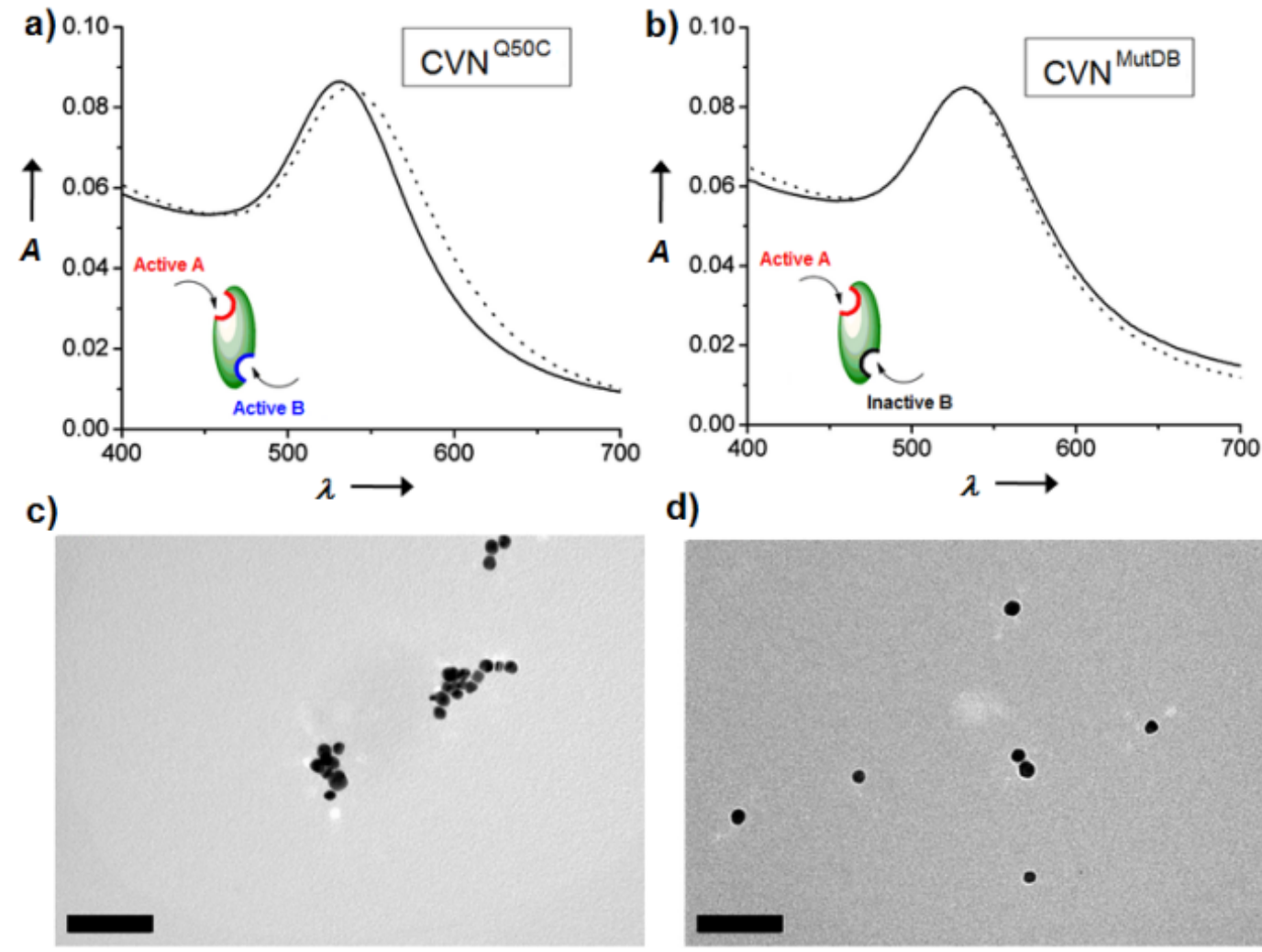

d)

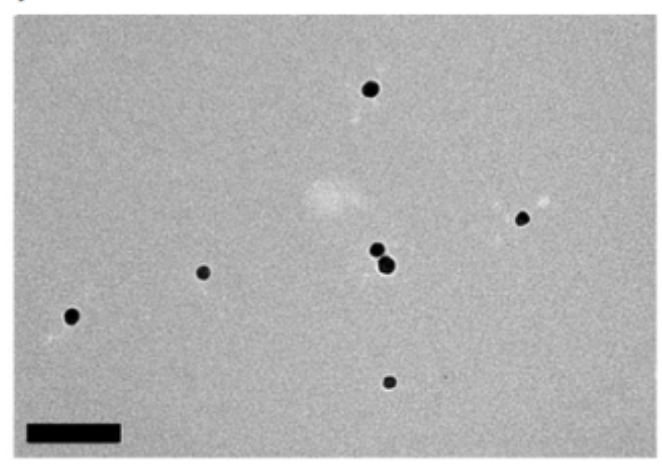

Figure 5.2 UV-vis spectra of GNP-M3 before (solid line) and after (dotted line) treatment with a) $\mathrm{CVN}^{\mathrm{Q} 50 \mathrm{C}}$ and b) $\mathrm{CVN}^{\mathrm{MutDB}}$. TEM micrographs of GNP-M3 treated with c) $\mathrm{CVN}^{\mathrm{Q} 50 \mathrm{C}}$ and d) $\mathrm{CVN}^{\mathrm{MutDB}}$. Scale bars: $100 \mathrm{~nm}$.

The binding affinities of the GNPs to the CV-N variants were evaluated using a recently developed fluorescence-based competition assay. ${ }^{[9]}$ In the experiment, free ligand competitor (Man2 for GNP-M2, Man3 for GNP-M3) together with varying concentrations of GNP-M2 or GNP-M3 was incubated at a fixed concentration of Cy5-CVN ${ }^{\mathrm{Q} 50 \mathrm{C}}$, specifically Cy5-labeled $\mathrm{CVN}^{\mathrm{Q} 50 \mathrm{C}}$ (Figure 5.3a, see experimental 
section for details). The solution was centrifuged to remove all GNPs and the fluorescence intensity of the supernatant was measured. The difference in fluorescence intensity of $\mathrm{Cy} 5-\mathrm{CVN}{ }^{\mathrm{Q} 50 \mathrm{C}}$ before and after incubation with GNPs corresponds to the amount of the bound $\mathrm{CVN}^{\mathrm{Q} 50 \mathrm{C}}$. Concentration response curves for GNP-M2 or GNPM3 permit the determination of $\mathrm{IC}_{50}$ values (Figure 5.3c).

a)

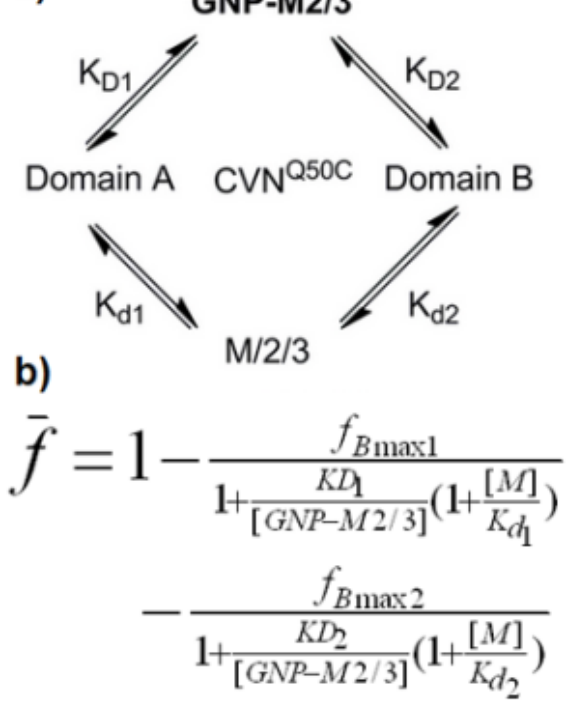

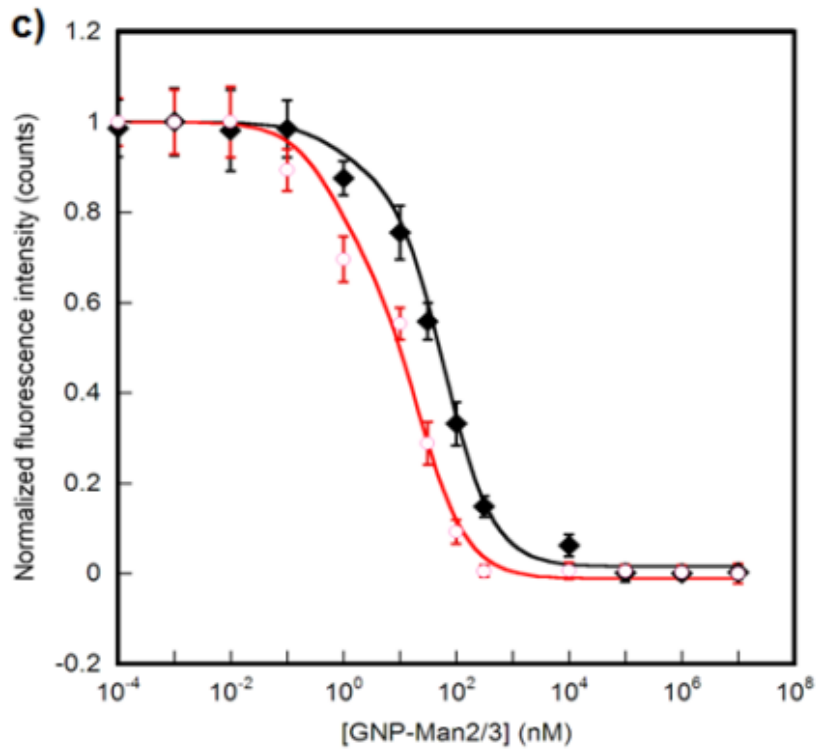

Figure 5.3 Fluorescence competition assay. a) Schematic representation of binding scenario. b) Modified Cheng-Prusoff equation based on a competitive two site binding model, where $[\mathrm{M}]$ is the concentration of the free ligand, and $\mathrm{K}_{\mathrm{d} 1}$ and $\mathrm{K}_{\mathrm{d} 2}$ are the dissociation constants of the free ligand for the glycan-binding sites on Domains A and $\mathrm{B}$ of $\mathrm{CVN}^{\mathrm{Q} 50 \mathrm{C}}$, respectively. The data were fitted using the maximum bound fractions, $\mathrm{f}_{\mathrm{B} \max 1}$ and $\mathrm{f}_{\mathrm{Bmax} 2}$, corresponding to the two binding sites, and dissociation constants $\mathrm{K}_{\mathrm{D} 1}$ and $\mathrm{K}_{\mathrm{D} 2}$ as adjustable parameters. c) Concentration response curves of GNP-M2 and GNP-M3. 
In order to extract the binding constants of GNPs with $\mathrm{CVN}^{\mathrm{Q} 50 \mathrm{C}}$, it is necessary to know the $\mathrm{K}_{\mathrm{d}}$ values of the monomeric ligands, Man2 and Man3, with $\mathrm{CVN}^{\mathrm{Q} 50 \mathrm{C}}$. These values were determined by ITC and the dissociation constants, $\mathrm{K}_{\mathrm{d} 1}$ (glycan-binding site on Domain A) and $\mathrm{K}_{\mathrm{d} 2}$ (glycan-binding site on Domain B), were calculated based on a two-site binding model (see Figure 5.4). Values for $\mathrm{K}_{\mathrm{d} 1}$ and $\mathrm{K}_{\mathrm{d} 2}$ of $700 \mu \mathrm{M}$ and $64 \mu \mathrm{M}$ for Man2, and 3.4 $\mu \mathrm{M}$ and $43 \mu \mathrm{M}$ for Man3, respectively, were calculated (Table 5.1). These values agree well with our previous observation that slightly stronger binding of Man2 to the site on Domain B than to that on Domain A occurs, while the opposite is true for Man3. ${ }^{[3 a, 3 c, 14]}$ These data, together with the $\mathrm{IC}_{50}$ values determined from the data shown in Figure $5.3 \mathrm{c}$, were then used to calculate the apparent dissociation constants for the site on Domain $\mathrm{B}, \mathrm{K}_{\mathrm{D} 1}$ and $\mathrm{K}_{\mathrm{D} 2}$, based on a two binding site model (Figure 5.3b). 
(a)

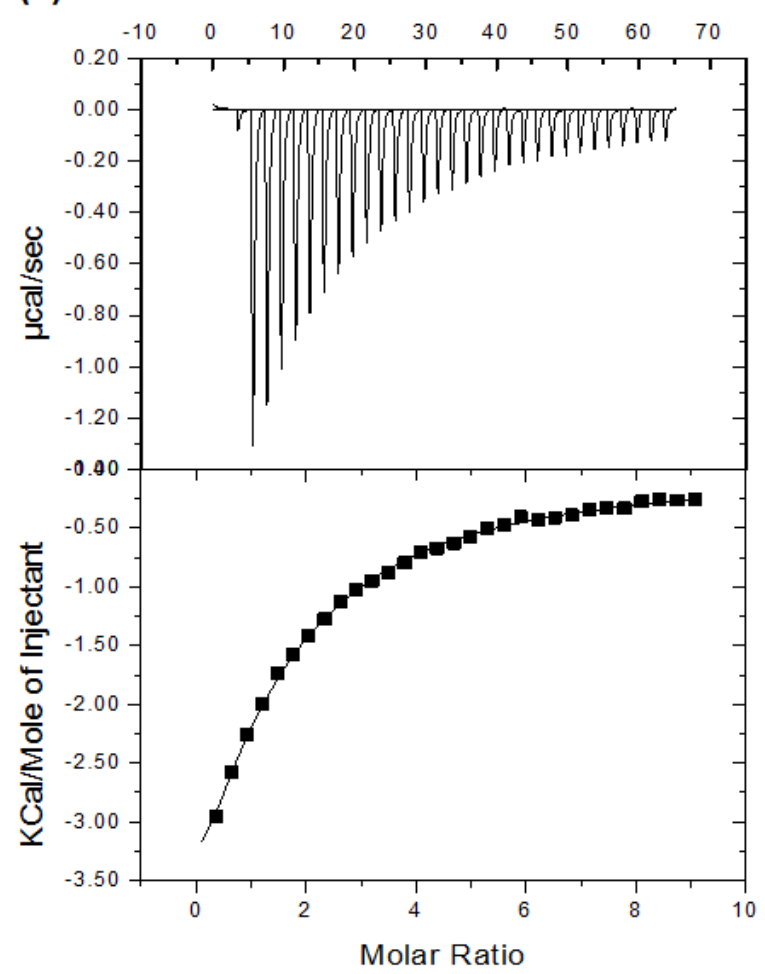

(b)

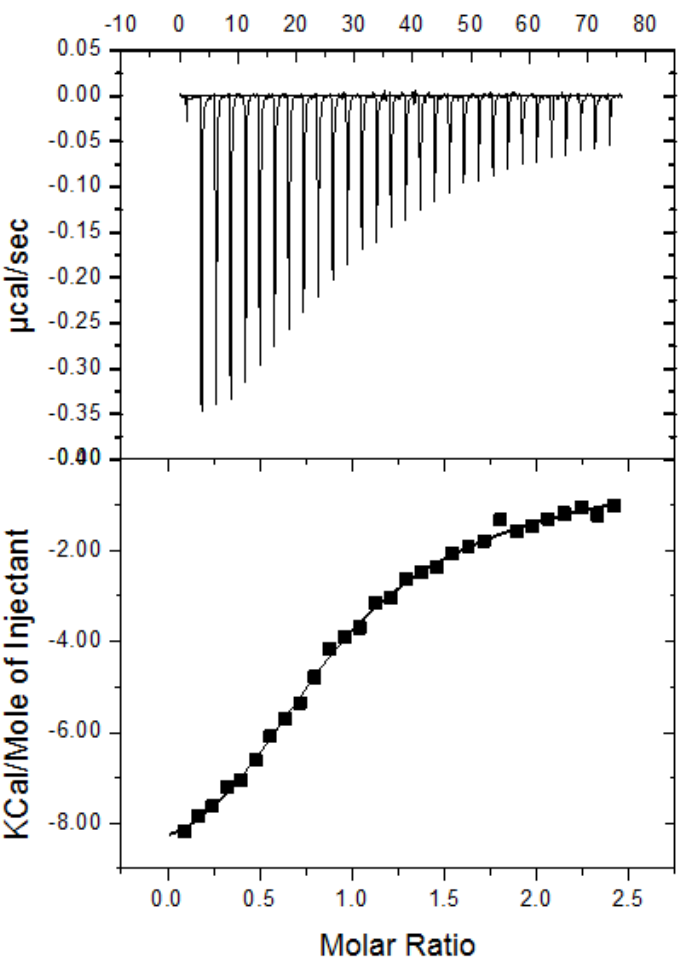

Figure 5.4 Calorimetric titration of a) $\mathrm{CVN}^{\mathrm{Q} 50 \mathrm{C}}(35 \mu \mathrm{M})$ with $\mathrm{Man} 2(1.5 \mathrm{mM})$, and b) $\mathrm{CVN}^{\mathrm{Q} 50 \mathrm{C}}(50 \mu \mathrm{M})$ with Man3 $(0.64 \mathrm{mM})$ at $30{ }^{\circ} \mathrm{C}$. The raw data were obtained for 30 and 20 automatic injections, respectively. The integrated curves show experimental points ( $\square$ ) and the best fit (-). The buffer was $50 \mathrm{mM}$ sodium phosphate, $200 \mathrm{mM}$ $\mathrm{NaCl}, \mathrm{pH} 7.5$, containing $0.02 \% \mathrm{NaN}_{3}$.

The data summarized in Table 5.1 demonstrate that both GNPs, GNP-M2 and GNPM3, exhibit an affinity enhancement by several orders of magnitude compared to the affinities measured for the isolated, monomeric sugars interacting with $\mathrm{CVN}^{\mathrm{Q} 50 \mathrm{C}}$. Taking into account the number of ligands on the particles, i.e. considering the affinity/ligand, an increase up to several hundred times is still present for the AuNPbound glycan (Table 5.1). In addition, GNP-M3 exhibited a higher affinity than 
Table 5.1 Affinities for Man2/3 $\left(\mathrm{K}_{\mathrm{d}}\right)$ and GNP-M2/3 $\left(\mathrm{K}_{\mathrm{D}}\right)$ binding to $\mathrm{CVN}^{\mathrm{Q} 50 \mathrm{C}}$. Numbers in parentheses correspond to $\mathrm{EF}\left(=\mathrm{K}_{\mathrm{d} 1} / \mathrm{K}_{\mathrm{D} 1} \bullet\right.$ Number of ligands on GNP).

\begin{tabular}{|c|c|c|}
\hline Ligand & $\begin{array}{c}\mathrm{K}_{\mathrm{d} 1} \text { or } \mathrm{K}_{\mathrm{D} 1} \\
\text { (Domain A) }\end{array}$ & $\begin{array}{c}\mathrm{K}_{\mathrm{d} 2} \text { or } \mathrm{K}_{\mathrm{D} 2} \\
\text { (Domain B) }\end{array}$ \\
\hline Man2 & $700 \pm 50 \mu \mathrm{M}$ & $64 \pm 4 \mu \mathrm{M}$ \\
\hline GNP-M2 & $56.4 \pm 7 \mathrm{nM}(8.2)$ & $0.24 \pm 0.1 \mathrm{nM}(176)$ \\
\hline Man3 & $3.4 \pm 0.2 \mu \mathrm{M}$ & $43 \pm 2 \mu \mathrm{M}$ \\
\hline GNP-M3 & $0.011 \pm 0.007 \mathrm{nM}(309)$ & $11.8 \pm 2.3 \mathrm{nM}(3.6)$ \\
\hline
\end{tabular}

GNP-M2 for both domains. These results correlate well with the general affinity ranking of the free ligands Man2 and Man3, and are consistent with observations in our previous study with a different GNP-lectin system. ${ }^{[9]}$ Interestingly, for both GNPM2 and GNP-M3, the affinity enhancement is more pronounced for the better binding domain. For example, Man2 exhibits a higher affinity for the binding site on Domain B, and with GNP-M2, the affinity enhancement factor (EF) is 178 for the Domain B site vs. 8.3 for the Domain A site (Table 5.1). For GNP-M3, on the other hand, the opposite was observed that the EF is higher for the Domain A site (340) than for the Domain B site (3.8).

\section{Conclusion}

In conclusion, we have successfully grafted low-mannose ligands onto AuNPs via an efficient photocoupling reaction. The resulting GNPs interacted with the CV-N 
variants $\mathrm{CVN}^{\mathrm{MutDB}}$ and $\mathrm{CVN}^{\mathrm{Q} 50 \mathrm{C}}$ in a manner that is consistent with the expected behavior of one- and two-site binders. Crosslinked complexes and aggregates were observed when GNP-M3 was treated with the two-site $\mathrm{CVN}^{\mathrm{Q} 50 \mathrm{C}}$ while only single particles were seen after treatment with the single-site variant $\mathrm{CVN}^{\mathrm{MutDB}}$. Furthermore, these GNPs exhibited significantly higher affinity towards the CV-N lectins, compared to the free glycan ligands, demonstrating that AuNPs serve as an efficient multivalent scaffold that significantly enhances the apparent affinity. This affinity enhancement compares well with that of other synthetic multivalent ligands. Therefore, a general strategy can be envisioned which uses simple glycans, rather than large and complex sugars, for grafting onto a multivalent scaffold for affinity amplification. These types of approaches will aid in development of effective new glyconanomaterials for diagnostic and therapeutic applications. 


\subsection{Labeling Native Glycans with Dye-doped Silica Nanoparticles}

\section{Introduction}

A major challenge in bioanalysis, including high-throughput screening, is the means to effectively display the outcome of the ligand-receptor interactions. Fluorescence is by far the most commonly used detection method, which requires the conjugation of a fluorescent tag to the ligand to be studied..$^{211-213}$ Among the fluorescent tags, organic dyes continue to be the label of choice due to the availability of a wide variety of commercial products of diverse structures, functionalities, solubility, and spectral properties. A major drawback of organic dyes, however, is their relatively poor photostability. When exposed to light, organic dyes can photobleach and lead to a decrease in fluorescence intensity. A clever solution to this problem is to entrap the fluorescent dye inside a solid matrix, for example silica nanoparticles. ${ }^{214}$ The nanoparticle protects the dye molecules from being directly exposed to the environmental oxygen, and thus greatly enhances the photostability of the entrapped dye. ${ }^{215}$ Furthermore, because a large number of dye molecules can be embedded inside a nanoparticle, high fluorescence emission can be obtained, the intensity of which exceeds the dye molecule itself or even quantum dots. ${ }^{216-219}$ In addition, silica nanoparticles are biocompatible and of low toxicity. ${ }^{3,84}$ Uniformly sized silica nanoparticles can be readily prepared from inexpensive starting materials following simple synthetic procedures. ${ }^{162}$ 
Ligand labeling often requires a robust conjugation method where the labeling agent can be covalently attached to the ligand. For many biomolecules, this can be conveniently accomplished, for example, by using a commercial kit containing chemically-derivatized labeling agents. For ligands that lack functional groups or are difficult to derivatize, the task of labeling can be complex and challenging. We have developed a general coupling chemistry, based on perfluorophenyl azides (PFPAs), that can conjugate a variety of molecules regardless of their chemical structures. ${ }^{126,140,181,220}$ Upon photochemical or thermal activation, PFPA is converted to a highly reactive singlet perfluorophenylnitrene which form covalent adducts with neighboring molecules by way of $\mathrm{CH}$ insertion and/or $\mathrm{C}=\mathrm{C}$ addition reactions. Therefore, by functionalizing FSNPs with PFPA, ligands can be conjugated to FSNPs via the photocoupling reaction of the surface PFPA. In this study, we present that PFPA-functionalized FSNPs can be used as highly efficient fluorescence labels for carbohydrate ligands. Carbohydrates are an important class of biomolecules involved in many important biorecognition processes including, for example, cell communication, immune responses, fertilization, and infections. ${ }^{57,61,64,178}$ Studies of these processes are, however, hampered by the high complexity of glycan structures and the lack of efficient bioanalytical tools. ${ }^{74,221-223}$ The present study seeks to address some of these challenges by developing a new method to efficiently label underivatized carbohydrates with FSNPs. We evaluate FSNP-labeled carbohydrates for their affinities with lectins. The synthesized glyco 
FSNPs are furthermore applied to image bacteria, and to probe carbohydrateprotein interactions on a lectin microarray.

\section{Experimental details}

Synthesis of FITC-doped silica nanoparticles FSNPS.

Fluorescein isothiocyanate ( $39 \mathrm{mg}, 0.10 \mathrm{mmol})$ was mixed with APTMS $(23 \mu \mathrm{L}, 0.10$ $\mathrm{mmol}$ ) in $100 \mathrm{~mL}$ of absolute ethanol, and was stirred at $42{ }^{\circ} \mathrm{C}$ for $24 \mathrm{~h}$ to yield the FITC-silane precursor as a bright yellow solution. The fluorescent nanoparticles were

synthesized following a modified protocol from the classic Stöber protocol, ${ }^{162}$ similar to what was previously described. ${ }^{125}$ The dye precursor solution $(5 \mathrm{~mL})$ was mixed with TEOS $(2.8 \mathrm{~mL})$, and the mixture was added to 200 proof absolute ethanol (34 $\mathrm{mL})$ followed by $\mathrm{NH}_{4} \mathrm{OH}(35 \%, 2.8 \mathrm{~mL})$. The reaction was allowed to proceed at room temperature for at least $8 \mathrm{~h}$ with vigorous stirring to yield a bright yellow colloidal solution. The particle diameters were determined by TEM and DLS (Figure 5.9 and 5.10 in Supporting Information)

Functionalization of silica nanoparticles with PFPA.

PFPA-silane $(80 \mathrm{mg})$, synthesized following a previously reported procedure, ${ }^{123}$ was added directly to the Stöber solution prepared above, and the mixture was stirred at room temperature overnight. The next day the mixture was brought to reflux while continuing stirring for $1 \mathrm{~h}$ at $\sim 78{ }^{\circ} \mathrm{C}$ to facilitate the covalent bond formation between PFPA-silane and the silica nanoparticles. ${ }^{125}$ The mixture was centrifuged at $8,000 \mathrm{rpm}$ 
for $10 \mathrm{~min}$, and the precipitate was redispersed in the fresh solvent by sonication. This centrifugation/redispersion procedure was repeated three times with ethanol and twice with acetone.

\section{Conjugation of carbohydrates onto FSNPS.}

Our previous reported procedure of coupling carbohydrates on gold nanoparticles was followed. ${ }^{181}$ The solution of PFPA-functionalized FSNPs in acetone $(20 \mathrm{mg} / \mathrm{mL}, 5$ $\mathrm{mL}$ ) was placed in a flat-bottom dish, and an aqueous solution of carbohydrate (10 $\mathrm{mg} / \mathrm{mL}, 1 \mathrm{~mL}$ ) was added. The mixture was covered with a 280 -nm long-path optical filter (WG-280, Schott Glass) and was irradiated with a 450-W medium pressure Hg lamp (Hanovia) for 10 min under vigorous stirring. Centrifugation of the solution at 8,000 rpm for 10 min separated the carbohydrate-attached FSNPs as precipitates. Excess carbohydrate was removed by membrane dialysis in water for 24 hours. The concentration of FSNP, $\sim 17.2 \mathrm{mg} / \mathrm{mL}$, was determined by drying the solution under reduced pressure for 3 hours and weighing.

Determination of carbohydrate density on glyco-FSNPs.

A previously developed colorimetric method was followed to determine the density of carbohydrates immobilized on FSNPs. ${ }^{181}$ Calibration curves were first obtained for each carbohydrate where carbohydrate solutions of various concentrations were incubated with anthrone/sulfuric acid and the absorbances at $620 \mathrm{~nm}$ were measured. ${ }^{155}$ A freshly-prepared anthrone solution in concentrated $\mathrm{H} 2 \mathrm{SO} 4(0.5 \mathrm{wt} \%, 1$ 
$\mathrm{mL})$ was added to a carbohydrate solution in water $(0.5 \mathrm{~mL})$ in an ice bath under stirring. The solution was then heated to $100{ }^{\circ} \mathrm{C}$ and stirred for $10 \mathrm{~min}$. After cooled to room temperature, the UV-vis spectra of the resulting solutions were recorded on a Perkin-Elmer Lambda 45 UV-vis spectrometer.

Carbohydrates coupled on nanoparticles were subjected to the same assay where solutions of the glyco-FSNPs in Milli-Q water $(30-50 \mu \mathrm{g} / 0.5 \mathrm{~mL})$ were treated with anthrone $/ \mathrm{H}_{2} \mathrm{SO}_{4}$. Background absorption due to FSNPs themselves was accounted for by treating FSNPs solution of the same concentration with anthrone/ $\mathrm{H}_{2} \mathrm{SO}_{4}$, and the absorbance at $620 \mathrm{~nm}$ was subtracted from that of the glyco-FSNPs. The amount of surface-bound carbohydrate was then computed from the corresponding calibration curve.

Binding with Con A and E. coli.

The binding affinity of FSNP-labeled Man, FSNP-Man, was evaluated using Con A and E. coli strain ORN178 and ORN208 according to the following procedure. FSNPMan $(2.5 \mathrm{mg})$ were incubated in a $\mathrm{pH}$ 7.2 HEPES buffer solution $(1 \mathrm{~mL}, 10 \mathrm{mM})$ containing 3\% BSA for $30 \mathrm{~min}$, centrifuged, and the particles were incubated in the pH 7.2 HEPES solution without BSA for another $20 \mathrm{~min}$. The nanoparticles were subsequently treated with a solution of Con A in HEPES buffer $(1 \mathrm{~mL}, 10 \mu \mathrm{g} / \mathrm{mL})$ containing $\mathrm{MnCl}_{2}(1 \mathrm{mM})$ and $\mathrm{CaCl}_{2}(1 \mathrm{mM})$, or E. coli solution for 1 hour while shaking. In cases where aggregation was induced after binding with Con A, the 
suspension was transferred to a centrifuge tube and was centrifuged at 8,000 rpm for $10 \mathrm{~min}$.

Fabrication of lectin microarrays.

Aldehyde-coated glass slides were prepared following a reported procedure. ${ }^{224}$ Piranha-cleaned glass slides were treated with a solution of 3-(trimethoxysilyl)butyl aldehyde in toluene $(2 \mathrm{mM})$ for 4 hours, rinsed with toluene and dried with $\mathrm{N}_{2}$. Solutions of lectins were prepared in $\mathrm{pH} 7.4$ phosphate-buffered saline (PBS) at varying concentrations of $0.1-1 \mathrm{mg} / \mathrm{mL}$ with $40 \%$ glycerol added to prevent evaporation of the liquid droplets after printing. Con A and SBA were then printed onto the aldehyde-functionalized glass slide using a robotic printer (BioOdyssey Calligrapher miniarrayer; Bio-Rad Laboratories, Inc.). The glass slides were then incubated in a humid chamber ( $80 \%$ humidity) at $25{ }^{\circ} \mathrm{C}$ for $3 \mathrm{~h}$ to facilitate the immobilization of the lectins. After incubation, the blocking solution of BSA in $\mathrm{pH}$ 7.4 PBS buffer (1\%) was added and the slides were incubated for $1 \mathrm{~h}$, rinsed with the PBS buffer and was dried with $\mathrm{N}_{2}$.

Microarray assay and fluorescence imaging.

The lectin microarrays were incubated in the solution of glyco FSNPs in HEPES (2.5 $\mathrm{mg} / \mathrm{mL}$ ) for $2 \mathrm{~h}$, and were then gently rinsed with the HEPES buffer 3 times and dried. The slides were scanned under a microarray scanner (GenePix 4000B, Molecular 
Devices, Inc) at excitation of $532 \mathrm{~nm}$. The fluorescent images were recorded and the data were analyzed using the supplied software (Axon GenePix Pro 5.1).

\section{Results and discussion}

(a)

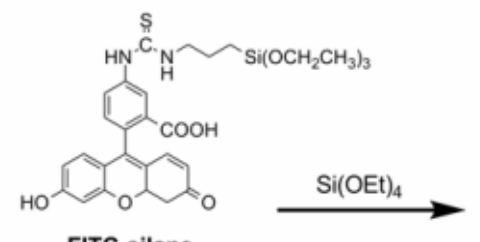

FITC-silane
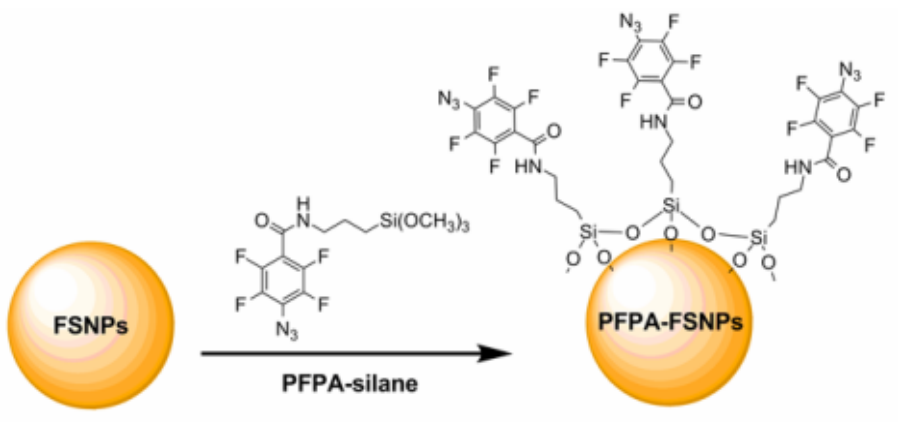

(b)

Glycan 1 Glycan 2

- 0
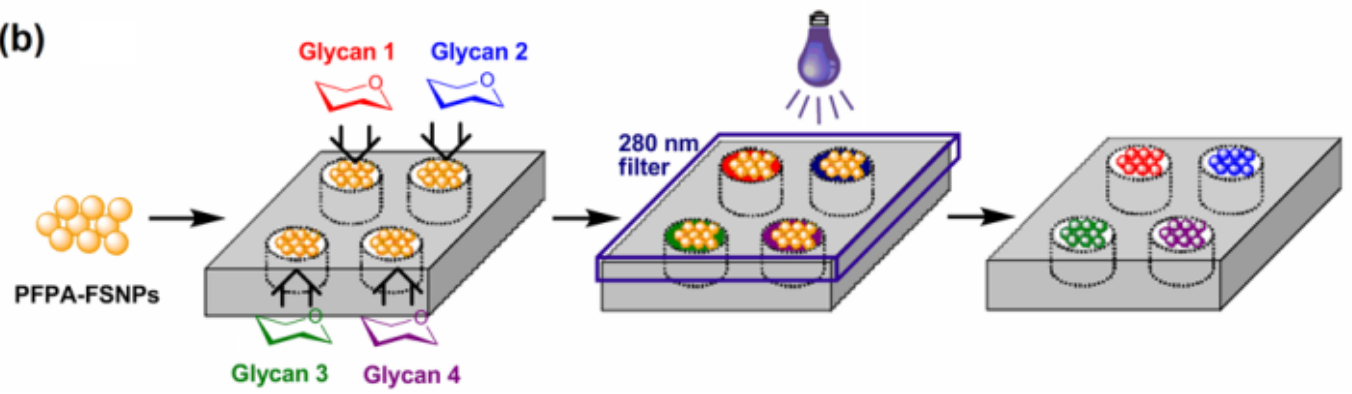

Figure 5.5 (a) Synthesis of PFPA-functionalized FSNPs, and (b) high-throughput synthesis of glyco FSNPs.

Fluorescein (FITC)-doped silica nanoparticles were synthesized using a modified Stöber method. ${ }^{162}$ Fluorescein isothiocyanate was silanized with 3aminopropyltriethoxysilane yielded FITC-silane (Figure 5.5a, which was then copolymerized with tetraethyl orthosilicate. ${ }^{214,217}$ The resulting FSNPs, $\sim 100 \mathrm{~nm}$ in diameter, showed intense fluorescence even at low particle concentrations . To test whether the entrapped FITC can withstand the UV irradiation condition used for 
the photocoupling reaction, a solution of FSNPs was irradiated with a $450-\mathrm{W}$ medium-pressure $\mathrm{Hg}$ lamp for $10 \mathrm{~min}$. The resulting solution remained highly fluorescent, and the fluorescence intensity decreased only to a small extent. On the other hand, when a solution of FITC was irradiated under the same condition, the fluorescence intensity was reduced to about $50 \%$ of the original value (Figure 5.11 and 5.12 in Supporting Information) These results are consistent with the observations by others that the photostability is significantly improved when fluorescent dyes are embedded in silica nanoparticles. ${ }^{219,225}$

Next, FSNPs were functionalized with PFPA by treating the as-prepared FSNPs with PFPA-silane (Figure 5.5a). ${ }^{125}$ To covalently label carbohydrates with FSNPs, our previously established procedure for coupling carbohydrates on gold nanoparticles was followed. ${ }^{155,181}$ In the process, a solution containing the carbohydrate and FSNPs was irradiated with a medium-pressure Hg lamp for 10 min (Figure 5.5b). Excess reagents were removed by dialysis, and the resulting glyco FSNPs showed excellent water solubility and high fluorescence emission intensity. The density of the immobilized carbohydrate was determined using a previously developed colorimetric assay, ${ }^{181}$ from which the coupling yields were calculated. The results showed that the photocoupling reaction was highly efficient. The coupling yield, ranging from $36 \%$ to $54 \%$ (Table 5.2 in Supporting Information), increased with the size of the carbohydrate, a result that is consistent with our previous study using gold nanoparticles. ${ }^{181}$ 
The labeling process is well adaptable for high-throughput where the photocoupling step can be performed in parallel. A pilot study was carried out where four micro-vials containing PFPA-FSNPs and four different carbohydrates were photoactivated simultaneously. The products showed successful conjugation of each carbohydrate on the FSNPs. The number of reaction wells can be further increased by using the microarray technology to enable a rapid parallel synthesis of larger libraries of FSNP-labeled ligands.
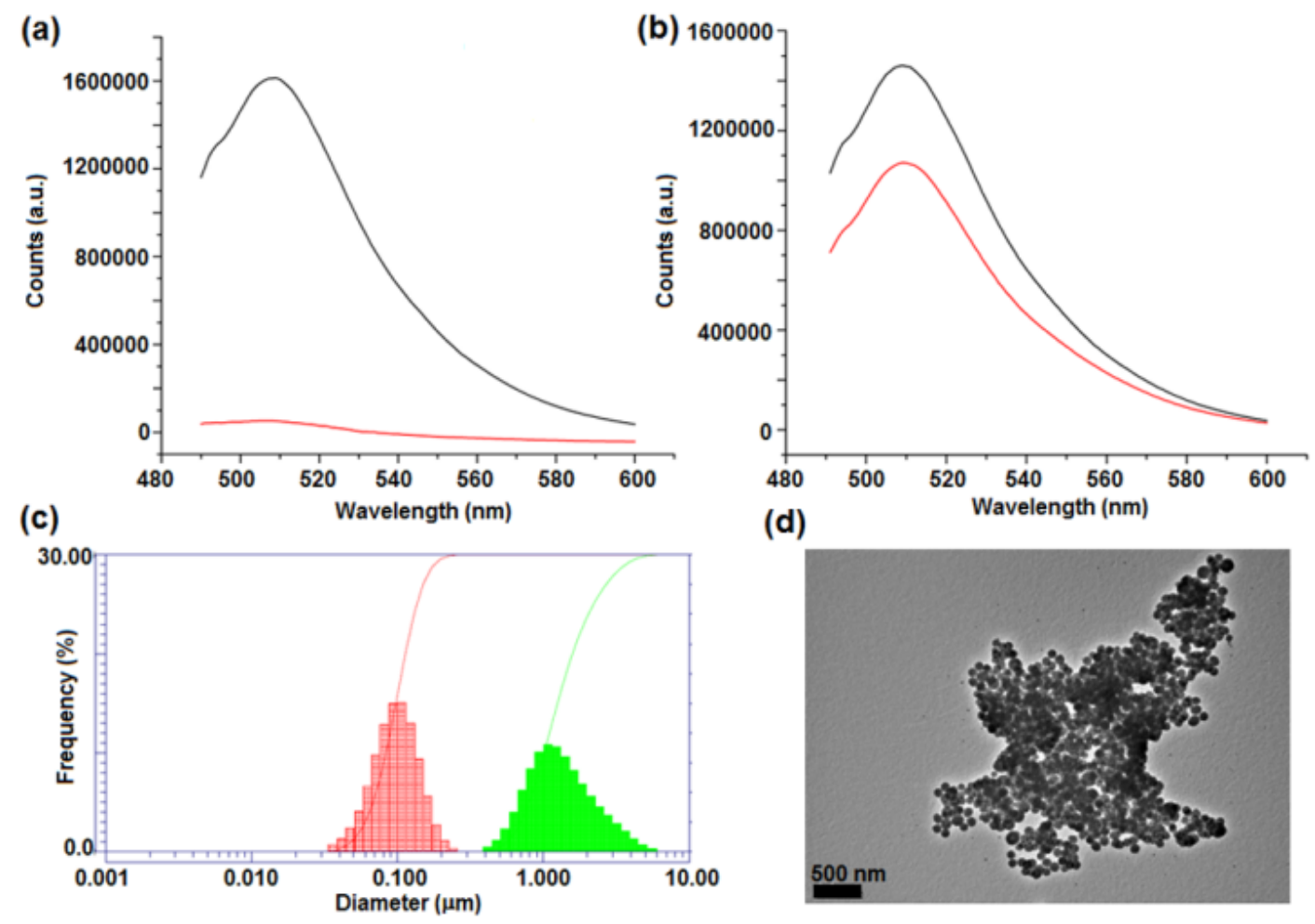

(d)

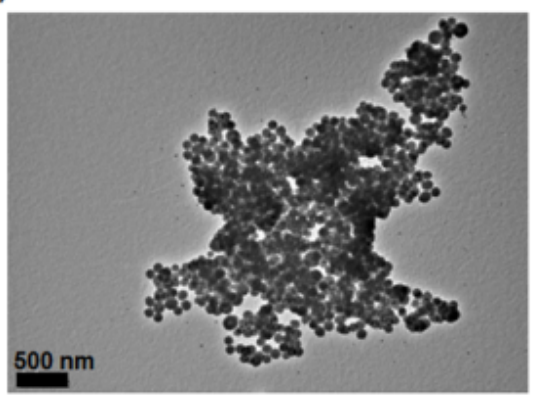

Figure 5.6 Fluorescence spectra of (a) FSNP-Man and (b) FSNP-Gal before (black lines) and after (red lines) incubating with Con A; (c) DLS of Man-FSNPs before (red) and after (green) binding with Con A; (d) TEM image of Man-FSNPs after treating with Con A. 
The bioactivity of FSNP-labeled carbohydrates was tested in the following studies. D-Mannose labeled with FSNP, FSNP-Man, was treated with Concanavalin A (Con A), a well-studied lectin which exhibits specific affinity to $\alpha$-Dmannopyranoside, $\alpha$-D-glucopyranoside, and their derivatives. ${ }^{114,183}$ At $\mathrm{pH}>7$, Con A exists as a tetramer, inducing significant nanoparticle agglomeration upon binding to Man-containing ligands. ${ }^{181}$ When FSNP-Man was incubated with Con A for 1 hour, the fluorescence intensity of the solution decreased drastically (Figure 5.6a). Precipitates were observed where the nanoparticles agglomerated into clusters (Figure 5.6d). In contrast, when FSNP-labeled D-galactose, FSNP-Gal, was treated with Con A following the same procedure, the fluorescence intensity decreased only slightly (Figure 5.6b). The small intensity decrease is likely due to the nonspecific adsorption of Con A on the nanoparticles. The FSNP-Man-Con A aggregates were additionally examined by dynamic light scattering (DLS), which showed, on the average, a 10-time increase in the particle size in comparison to FSNP-Man (Figure 5.6c). 

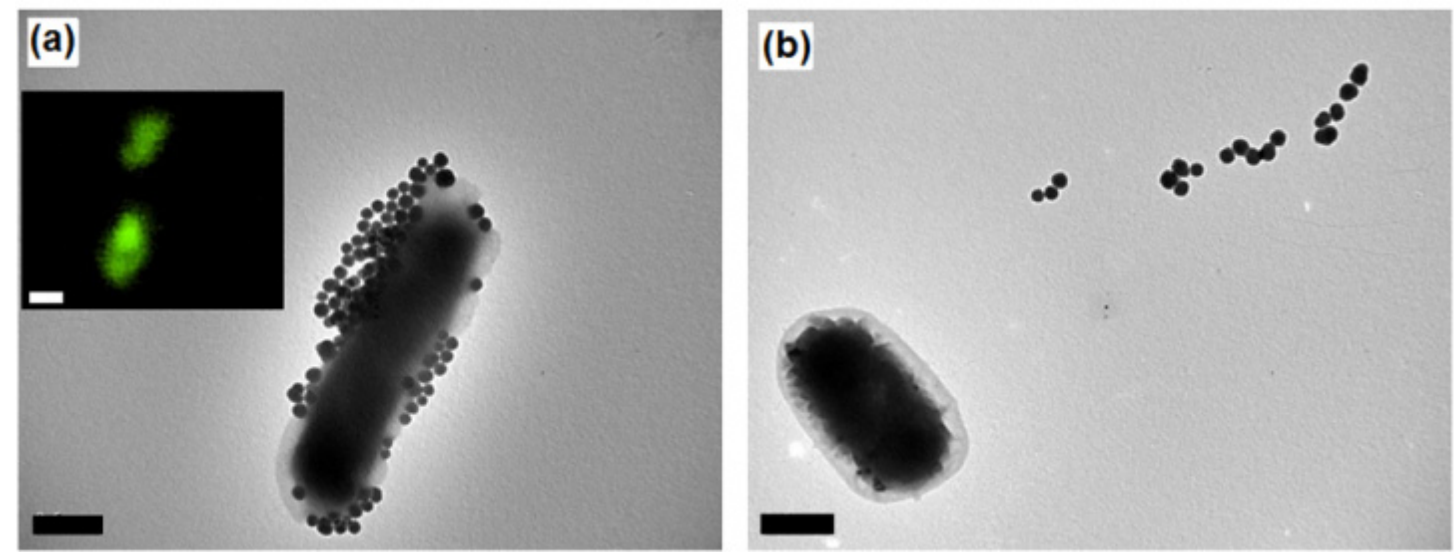

Figure 5.7 TEM images after FSNP-Man was treated with E. coli strain ORN178 (a), or ORN208 (b). Insert: fluorescence image of the corresponding sample. Scale bars: $500 \mathrm{~nm}$.

The utility of the FSNP labeling technique was next investigated by applying glyco FSNPs in imaging, and for studying glycan-lectin interactions on a lectin microarray. In the first experiment, FSNP-Man was treated with E. coli bacteria strain ORN 178. This particular strain contains a Man-specific binding domain, i.e., the FimH lectin, on type 1 pili. ${ }^{226,227}$ The TEM image of the resulting solution displays a large number of FSNP-Man on the E. coli (Figure 5.7a), which can be attributed to the multivalent interactions between Man ligands on the FSNP-Man with the FimH lectin on the bacteria. ${ }^{140}$ The strong interaction was further confirmed by fluorescence imaging where intense fluorescence was observed on the bacteria (Figure 5.7a insert). In contrast, when FSNP-Man was treated with $E$. coli strain ORN208 that is deficient of the Man-binding FimH protein, no fluorescence was observed on the bacteria. In fact, almost no nanoparticles were attached to the bacteria surface (Figure 5.7b). 


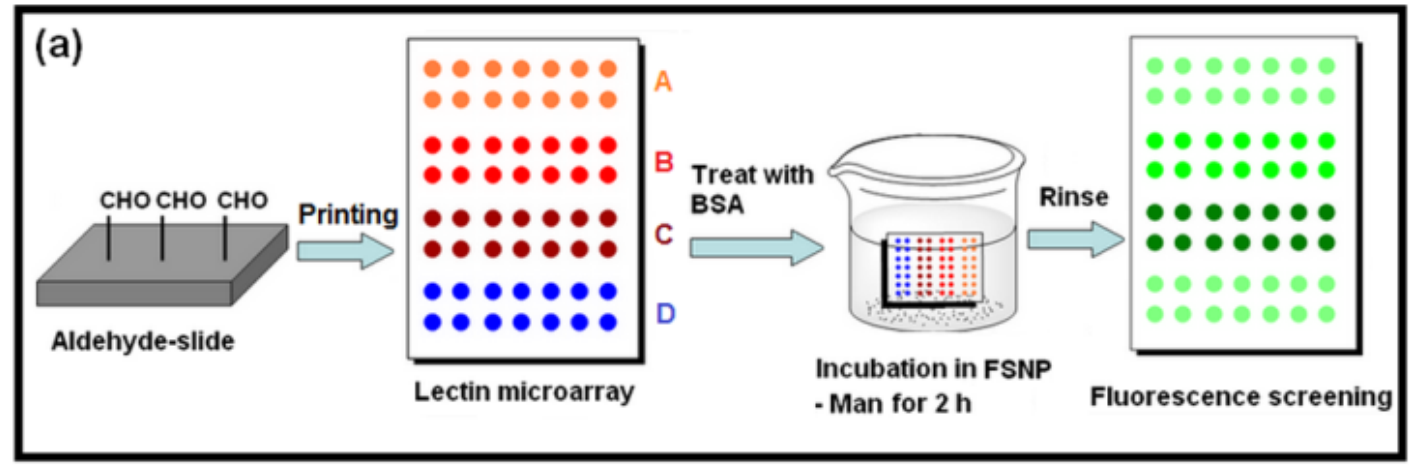

(b)

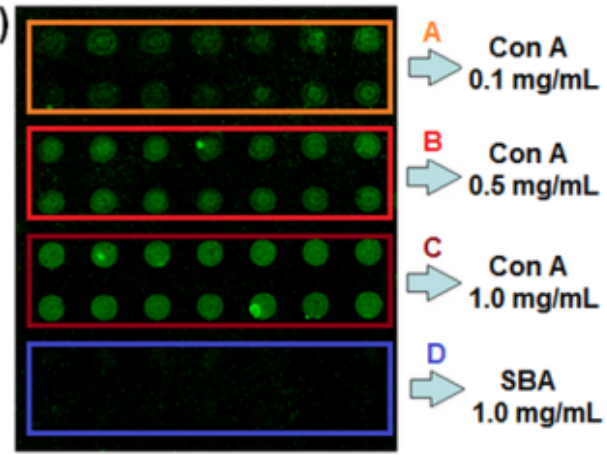

(c)

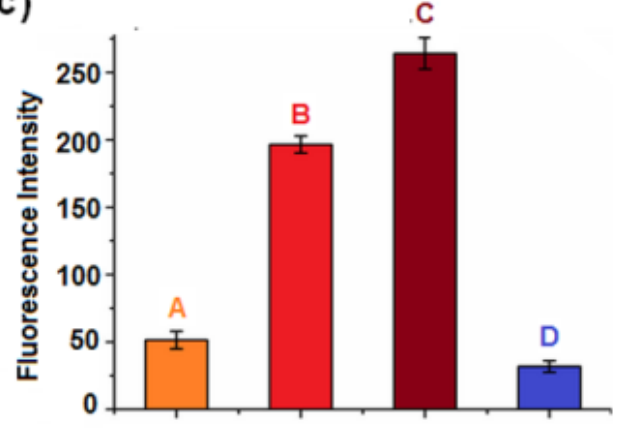

Figure 5.8 (a) Preparation of lectin microarray, incubation with FSNP-Man, and fluorescence imaging. (b) Fluorescence image and (c) fluorescence intensities of lectin microarray after treating with FSNP-Man. Each data point was the average of the 7 spots on the microarray.

The FSNP-labeled carbohydrates were next employed to study carbohydrate-lectin interactions on a lectin microarray. The lectin microarray was fabricated on aldehyde-functionalized glass slides following the established literature procedure. $^{224}$ Solutions of Con A and soybean agglutinin (SBA, a non-Manbinding lectin) in $\mathrm{pH} 7.4$ phosphate-buffered saline (PBS) containing $40 \%$ glycerol were then printed on the aldehyde slides using a robotic printer. ${ }^{228}$ The lectin array slide, after blocking with bovine serum albumin (BSA), was incubated in the FSNP-Man solution for 2 hours (Figure 5.8a). As anticipated, fluorescence was 
observed on all Con A spots (Figure 5.8b). The relative fluorescence intensity on Con A were $\sim 10$ times higher than those on SBA at the printing concentration of 1 $\mathrm{mg} / \mathrm{mL}$ (Figure 5.8c). Even at $0.1 \mathrm{mg} / \mathrm{mL}$, the fluorescence intensity from Con A was still noticeably higher than that from SBA, although the spot quality deteriorated at lower printing concentrations. These results clearly demonstrate that the FSNP-labeled carbohydrates are highly suited for interrogating carbohydratelectin interactions on microarray. Due to the irreversible agglomeration upon the interactions, the binded nanoparticles were found difficult to be displaced by other ligands. However, in order to extensively evaluate the specificity and apparent affinity of glyco FSNPs to lectin microarray, we currently developed an alternative competition-based assay, with unlabelled ligands added with FSNPs during the incubation. The preliminary results showed the florescence intensity was highly affected by concentration of free ligand competitors, and calculated affinities of FSNPs were several orders of magnitudes higher than that of monovalent binding in solution.

\section{Conclusion}

In conclusion, a simple and general method was developed to label carbohydrates with dye-doped silica nanoparticles. The strategy applies to underivatized carbohydrate structures, thus avoiding complex synthesis and purification steps that are often involved in the chemical derivatization of these ligands. The labeling is highly efficient, and the resulting FSNP-labeled carbohydrates retained their binding affinity 
and selectivity towards lectins. The utility of this labeling technique was successfully demonstrated where FSNP-labeled carbohydrates were applied in bacteria detection and imaging, and in probing glycan-lectin interactions on microarray. These results illustrate that, although the labeling chemistry, i.e., the $\mathrm{CH}$ insertion reaction of PFPA, yields a random orientation of the attached ligands, the labeled ligands retain their binding selectivities nonetheless. Further, the labeling reaction may allow the exposure of all epitopes on the ligands, and thus a biased epitope selection can be avoided. The technique developed can in principle be readily applied to other biologically significant molecules including pharmaceuticals and metabolites. The advantage of this method resides in its generality and simplicity, where the labeling process employs a single labeling agent, PFPA-FSNP, and a uniform coupling chemistry. Ligands are labeled in their native forms without undergoing prior chemical derivatization. These features, combined with the straightforward preparation of dye-doped silica nanoparticles and the low material cost, may open up a myriad of opportunities for this technique in bioanalysis and diagnostic applications. 


\section{Supporting Information}

TEM and DLS characterization of FITC-doped SNPS

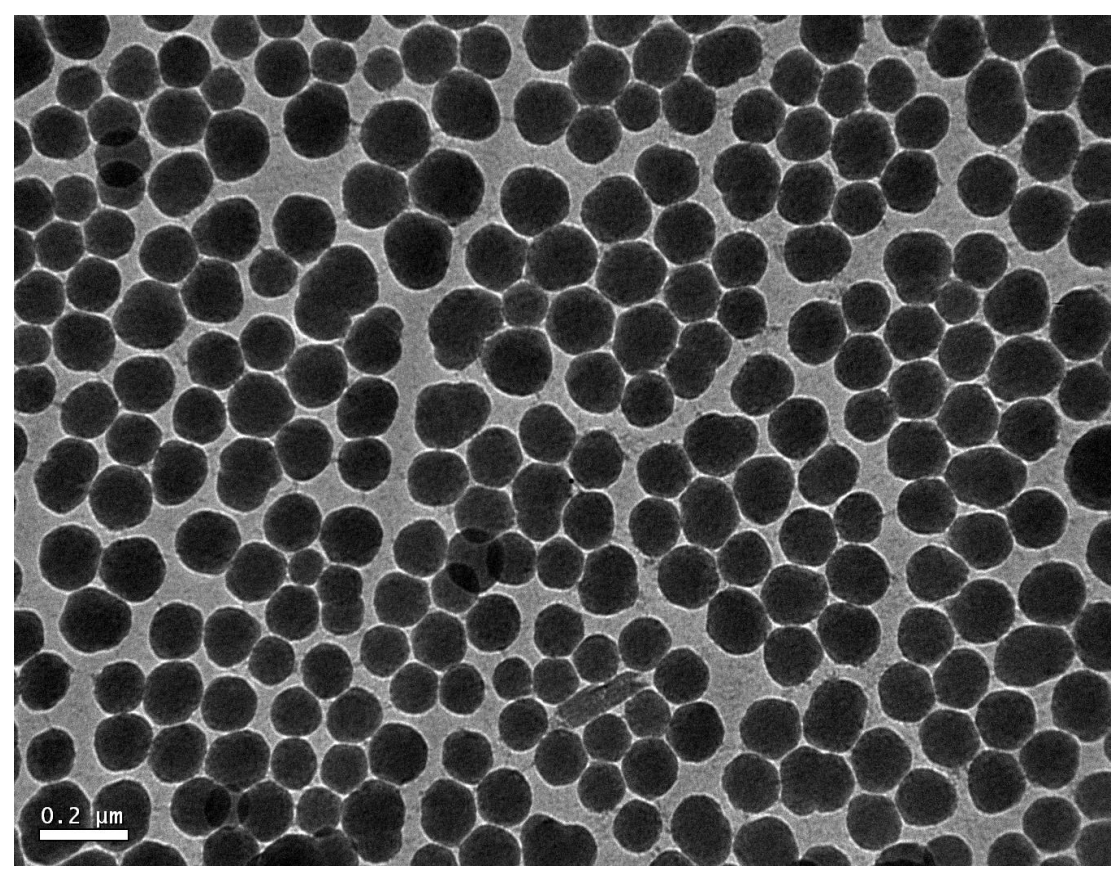

Figure 5.9 TEM image of FITC-doped SNPs. (Scale bar: $0.2 \mu \mathrm{m}$ )

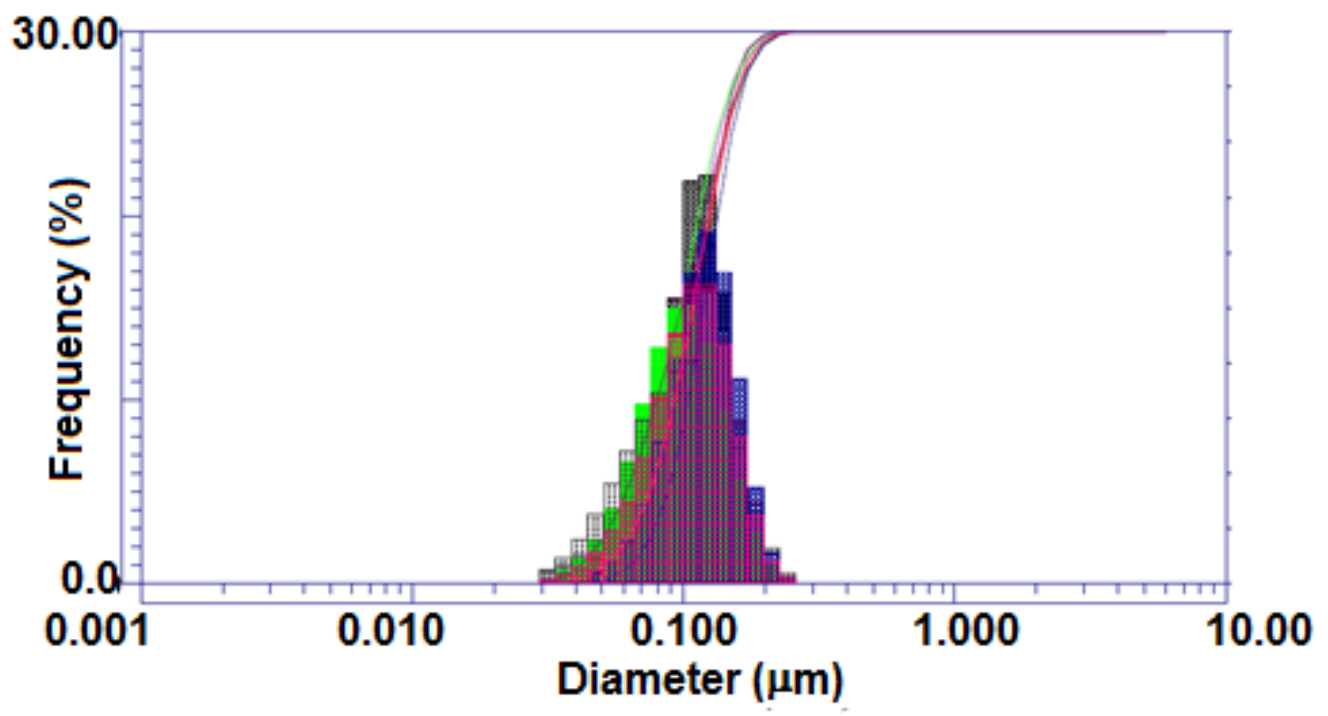

Figure 5.10 Size distribution of FITC-doped SNPs measured by DLS. 
Photo-stability of FITC-doped silica nanoparticles and FITC

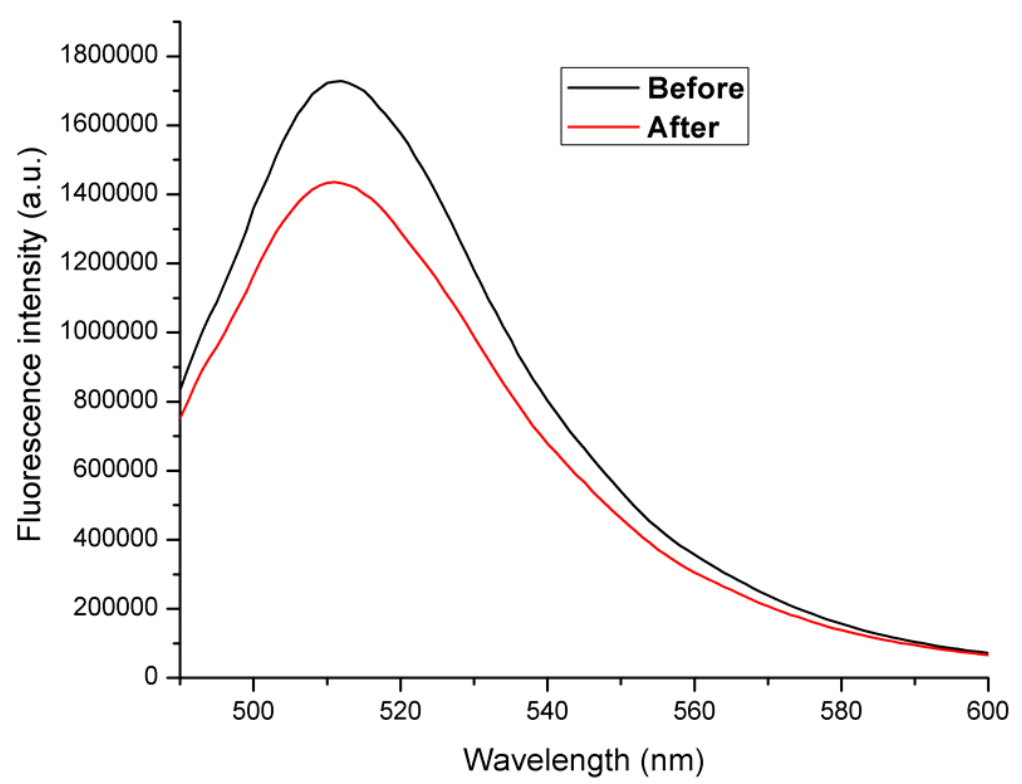

Figure 5.11 Fluorescence spectra of FITC-doped silica nanoparticles before and after UV irradiation for $10 \mathrm{~min}$.

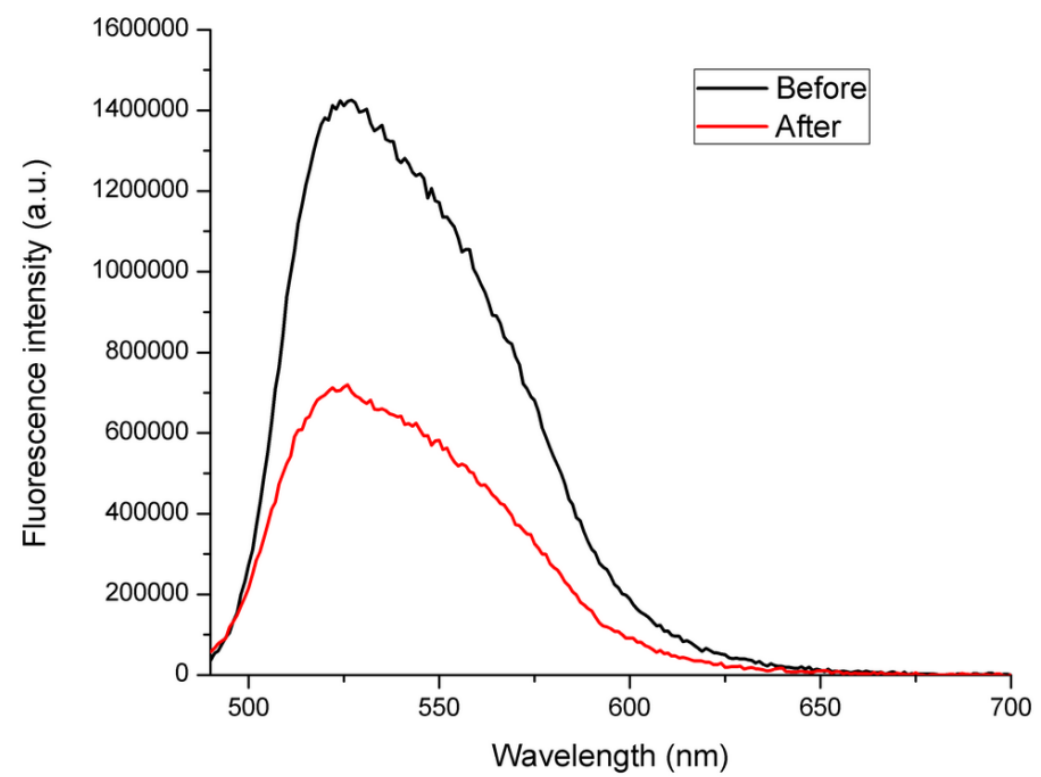

Figure 5.12 Fluorescence spectra of FITC before and after UV irradiation for $10 \mathrm{~min}$. 


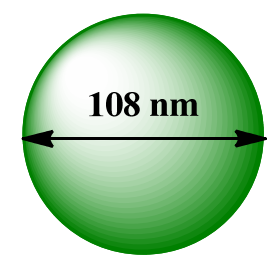

Surface area of each FSNP: $\mathrm{S}=\pi * \mathrm{~d}^{2}=36625 \mathrm{~nm}^{2}$

Volume of each FSNP: $\mathrm{V}=\pi^{*} \mathrm{~d}^{3} / 6=6.59 \times 10^{5} \mathrm{~nm}^{3}=6.59 \times 10^{-16} \mathrm{~cm}^{3}$

Density of FSNP $=2.3 \mathrm{~g} / \mathrm{cm}^{3}$

Concentration of FSNP $=20.61 \mathrm{mg} / \mathrm{mL}$

So in $1 \mathrm{~mL}$, No. of FSNP $=(0.02061 / 2.3) /\left(6.59 \times 10^{-16}\right)=1.36 \times 10^{13}$

By assuming the projection area of carbohydrate molecules, such as D-mannose $0.24 \mathrm{~nm}^{2}$, the number of maximal ligands occupied on each FSNP is:

$\mathrm{N}_{\text {Max }}=36625 / 0.24=1.53 \times 10^{5}$

The experimental number of ligands $\left(\mathrm{N}_{\mathrm{exp}}\right)$ was determined by anthrone- $\mathrm{H}_{2} \mathrm{SO}_{4}$ colorimetric measurements (using FSNP as background). The coupling yield was calculated as $\mathrm{N}_{\exp } / \mathrm{N}_{\mathrm{Max}} \mathrm{x} 100 \%$

Table 5.2 Ligand density and coupling yield of glyco FSNPs

\begin{tabular}{|c|c|c|c|}
\hline Carbohydrate ligand & $\begin{array}{c}\text { No. of ligand per } \\
\text { FSNP (x10 } \\
\text { experimental) }\end{array}$ & $\begin{array}{c}\text { No. of ligand per } \\
\text { FSNP (x10 } \\
\text { calculated) }\end{array}$ & $\begin{array}{c}\text { Coupling } \\
\text { yield (\%) }\end{array}$ \\
\hline $\begin{array}{c}\text { D-Mannose (Man) } \\
\begin{array}{c}\alpha-1,2-\text {-Mannobiose } \\
\text { (Man2) }\end{array}\end{array}$ & 5.52 & 15.3 & 36 \\
\hline $\begin{array}{c}\alpha-1,3-1,6-M a n n o t r i o s e \\
\text { (Man3) }\end{array}$ & 2.48 & 4.76 & 52 \\
\hline $\begin{array}{c}\text { D -Glucose (Glc) } \\
\text { Maltopentaose (Glc5) }\end{array}$ & 5.64 & 15.3 & 37 \\
\hline
\end{tabular}




\subsection{High-throughput Glycan Screening on Lectin Microarray}

Microarrays have become increasingly used in biological research for large-scale ligand profiling, owing to its features of high-throughput signal output and fast data analysis. Frequently, glycan microarrays are fabricated which are subsequently

employed to study glycan-lectin interactions using fluorescently-labeled lectins. ${ }^{51,80}$ An alternative configuration is to construct lectin microarrays which can then be probed with labeled glycans. Protein microarrays are more developed than glycan microarrays, and they can be fabricated using established procedures ${ }^{224,229}$ or acquired from commercial sources. ${ }^{230}$ Glycan labeling, however, can be challenging. Unlike proteins that can be readily labeled using commercially available kits, labeled glycans, on the other hand, are not easy to obtain. One strategy is to label glycoproteins. In the study done by Hirabayashi and coworkers, an glycan profiling technique was described based evanescent-field fluorescence detection strategy. The interactions between lectin microarrays fabricated on epoxy-derivatized slides and fluorescent Cy3-tagged glycoproteins, glycopeptides and tetramethylrhodamine (TMR)-labeled oligosaccharides were monitored, and the dissociation constants were also determined by competition assays. ${ }^{213}$

We have demonstrated that dye-doped silica nanoparticles provided an attractive platform to label glycans for the recognition of carbohydrate-binding proteins, lectins. Because multiple dye molecules are trapped inside the NPs, the resulting fluorescent nanoparticles are brighter than single dye molecule, and the fluorescent intensity is 
more stable towards photobleaching and environmental degradation. ${ }^{215,228}$ In the previous chapter, we demonstrate that the FSNP-labeled glycans are effective in probing lectin microarrays. In this chapter, a novel "super-microarray" design will be adapted where FSNP-labeled glycans will be used to study glycan-lectin interactions in high throughput. The super-microarray differs from the conventional microarray that it consists of multiple microarrays (Figure 5.13). By applying a poly(dimethyl siloxane) (PDMS) isolator, each microarray can be isolated and therefore different assays can be conducted in each individual microarray (Figure 5.13).

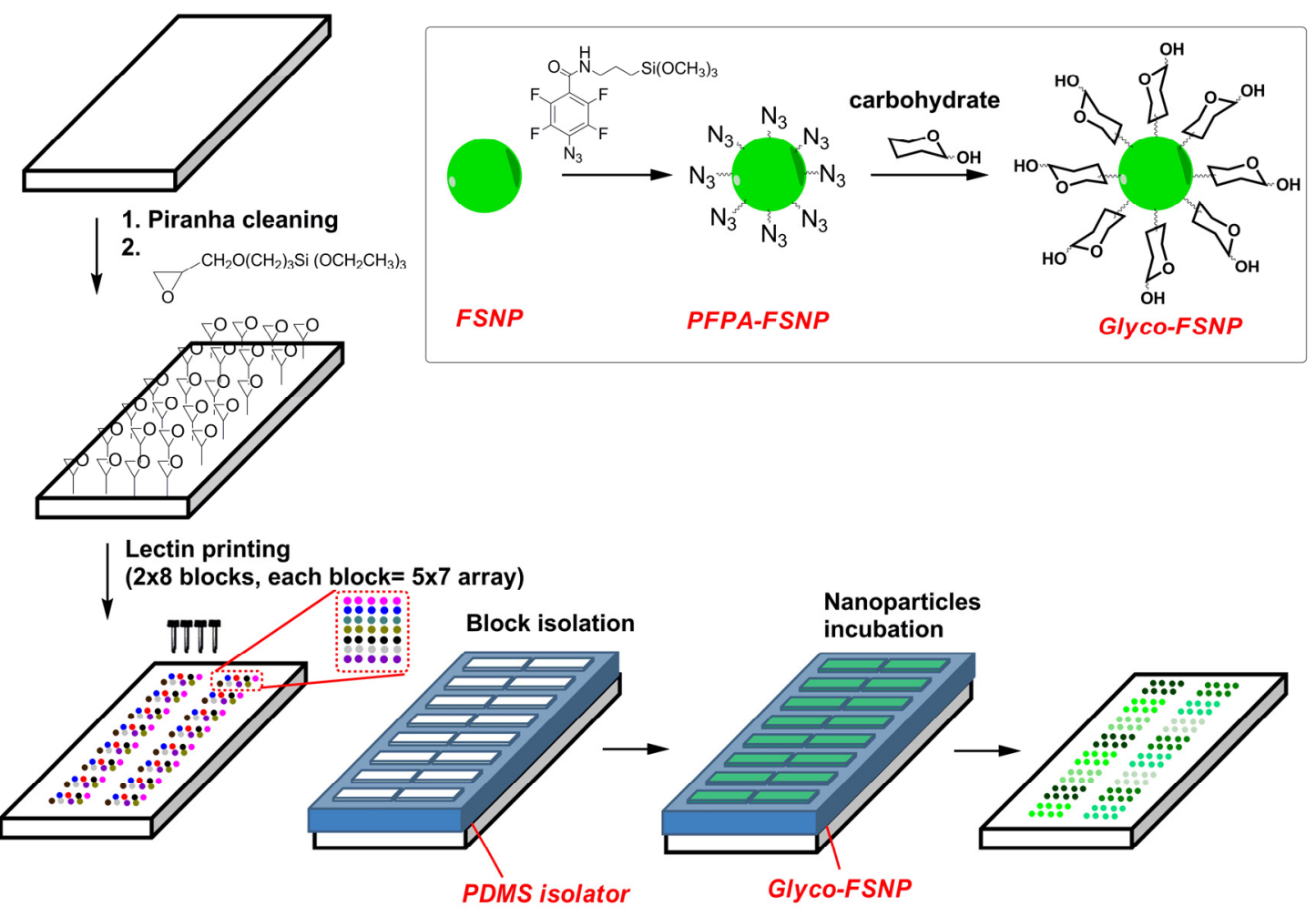

Figure 5.13 Schematics of lectin super-microarray fabrication, and the subsequent assays with glyco-FSNPs. 
The super-microarrays can be used for high throughput screening of ligands (Figure 5.14a) and for high throughput competition assays to determine binding affinity of glycan-lectin interactions (Figure 5.14b). Both applications will be demonstrated in this Chapter where FSNP-labeled glycans will be used on lectin supermicroarrays. Competition assays will be carried out simultaneously on a single super-microarray, from which $\mathrm{IC}_{50}$ values will be derived and the binding affinity calculated. This provides a high throughput method to determine binding affinity of glycan-lectin interactions.

(a) High-throughput Screening
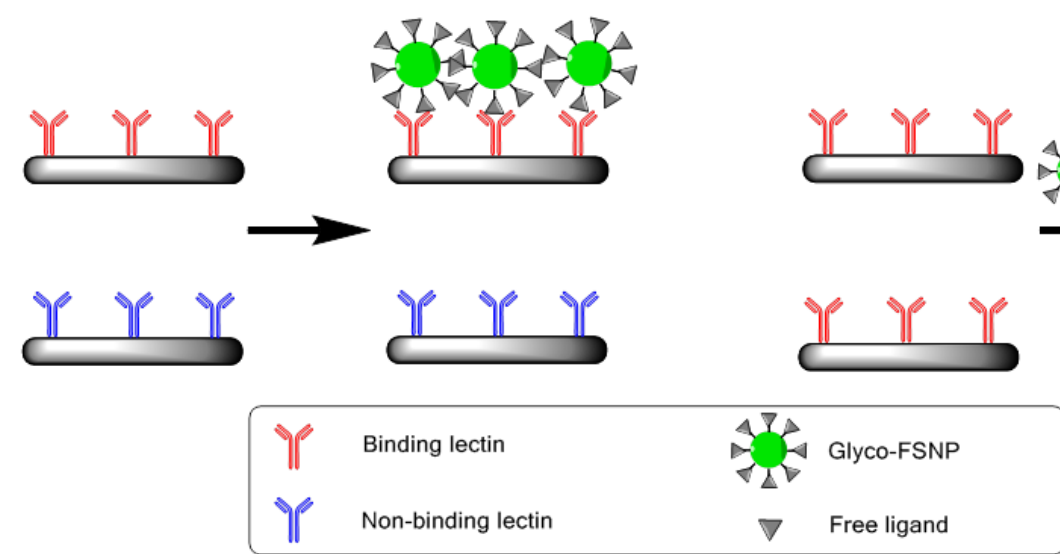

(b) Competition Assays

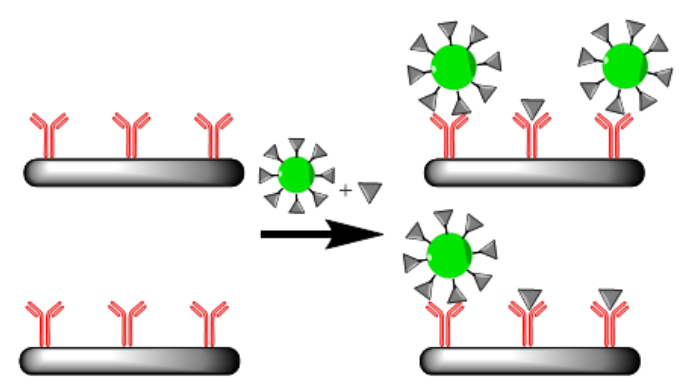

$\nabla \quad$ Free ligand

Figure 5.14 Schematics of (a) high-throughput screening, and (b) competition assay on super microarrays.

\section{Experimental details}

Preparation of FITC-doped glyconanoparticles.

See section $5.2^{166}$ 
Fabrication of lectin super-microarrays.

The lectin super-microarray was prepared on an epoxy-coated glass slide. Glass slides were firstly cleaned in Piranha solution (conc. $\mathrm{H}_{2} \mathrm{SO}_{4} / \mathrm{H}_{2} \mathrm{O}_{2}, 1: 1$, v:v), followed by soaking in a toluene solution of 3-glycidyloxypropyltrimethoxysilane (12.6 mM, $>95 \%$, TCI America; Portland, OR) for 4 hours, rinsed thoroughly with toluene and dried with nitrogen. Con A (Concanavalin A), BSA (Bovine serum albumin), SBA (Soybean Agglutinin), PNA (Peanut Agglutinin), BS-I (Bandeiraea simplicifolia) (Sigma-Aldrich; St. Louis, MO), DBA (Dolichos Biflorus Agglutinin), UEA (Ulex Europaeus Agglutinin I), WGA (Wheat Germ Agglutinin) and RCA (Ricinus Communis Agglutinin I) (Vector Laboratories; Burlingame, CA) were used as received. The concentration of Con A was determined spectrophotometrically at 280 $\mathrm{nm}$ using $\mathrm{A}^{1 \%}, 1 \mathrm{~cm}=13.7$ at $\mathrm{pH} 7.2 .{ }^{136} \mathrm{CV}-\mathrm{N}($ Cyanovirin- $\mathrm{N})$ and OAA (Oscillatoria Agardhii Agglutinin) wild-type lectins and $\mathrm{CN}-\mathrm{V}$ mutants $\mathrm{CVN}^{\mathrm{Q} 50 \mathrm{C}}$ and $\mathrm{CVN}^{\mathrm{MutDB}}$ were supplied by Professor Angela M. Gronenborn's research laboratory at University of Pittsburgh. Lectin solutions were prepared in phosphate-buffered saline at concentrations of $1 \mathrm{mg} / \mathrm{mL}$ (except PNA and RCA at $0.25 \mathrm{mg} / \mathrm{mL}$ ) with $40 \%$ glycerol added to prevent complete drying of the liquid droplets after printing. A volume of $0.3 \mathrm{~mL}$ lectin solution was added to the microtiter plate well and the solutions were spotted onto the epoxy-coated glass slides using a robotic printer (BioOdyssey Calligrapher Mini-arrayer; Bio-Rad Laboratories, Inc.) and capillary pin of $\sim 360 \mu \mathrm{M}$ size. Each spot was printed 5 times. Each lectin was printed with 5 duplicate spots. The glass slides were then incubated in a humid chamber $\left(80 \%\right.$ humidity) at $25{ }^{\circ} \mathrm{C}$ for 
$3 \mathrm{~h}$ to facilitate the immobilization of the lectins. A SecureSeal ${ }^{\mathrm{TM}}$ hybridization chambers sheet (Grace Bio-lab, Bend, OR) was carefully placed on the glass slide to create 16 individual wells. ${ }^{213}$ A blocking solution of BSA in pH 7.4 PBS buffer (1\%) was then added to each well and the slide was incubated at room temperature for $1 \mathrm{~h}$, rinsed with the PBS buffer and was dried with $\mathrm{N}_{2}$.

Treating supermicroarray with glyco-FSNP.

The lectin supermicroarrays were incubated in the solution of glyco FSNPs in HEPES $(1.5 \mathrm{mg} / \mathrm{mL})$ for $2 \mathrm{~h}$; for the competition assays, $1 \mathrm{nM}$ to $1 \mathrm{mM}$ free ligand Man2 was added in each well with FSNP-Man2 $(1.5 \mathrm{mg} / \mathrm{mL})$; and were then gently rinsed with the HEPES buffer containing $0.1 \%$ Tween 20 for 3 times and dried with $\mathrm{N}_{2}$. The slides were scanned under a microarray scanner (GenePix 4100A, Molecular Devices, Inc) at excitation of $532 \mathrm{~nm}$. The fluorescent images were recorded and the data were analyzed using the supplied software (Axon GenePix Pro 5.1).

\section{Results and discussion}

\section{Optimization of super-microarrays and assay conditions}

Lectin microarrays were prepared following a literature procedure by immobilizing lectins on epoxy-functionalized surfaces. ${ }^{224}$ Piranha-cleaned glass slides were treated with an epoxy-silane, GPTMS (Figure 5.13). Lectin solutions in PBS containing glycerol were then printed onto GPTMS-modified glass slides using a microarray printer. Multiple lectin microarrays were printed on the same glass slide where each 
microarray was separated by a 16-well PDMS isolator to give a super-microarray with 16 individual microarray on a single slide (Figure 5.13).

The lectin super-microarrays were tested for their ability for screening carbohydrate ligands. The carbohydrates were labeled with FITC-doped silica nanoparticles. We have demonstrated previously that dye-doped silica nanoparticles can efficiently label glycans. ${ }^{166}$ Because multiple dye molecules are trapped inside silica nanoparticles, the resulting FSNPs are brighter and the fluorescence is more stable than the free dye molecules as they are protected by the nanoparticles. ${ }^{215}$ FSNPs of $110 \mathrm{~nm}$ diameter were synthesized, ${ }^{166}$ and carbohydrate ligands were conjugated onto the fluorescent nanoparticles using the previously developed PFPA photocoupling chemistry to yield FSNP-labeled carbohydrates, glyco-FSNPs (Figure 5.13). ${ }^{125,166,181}$

The binding experiments were conducted by incubating buffer solutions containing glyco-FSNPs in each well on the lectin super-microarray. After rinsing with the fresh buffer and drying, the slide was subjected to a microarray scanner and the fluorescence image was recorded. The lectin printing concentration, the concentration and incubation time of glyco-FSNPs were varied to study the impact of these experimental conditions on the binding results. Figure 5.15a shows that when Man2FSNPs were incubated with Con A, the fluorescence intensity increased linearly with the Con A printing concentration from 0.1 to $2.5 \mathrm{mg} / \mathrm{mL}$. Similarly, the fluorescence intensity increased with the concentrations of Man2-FSNPs from 0.05 to $1.0 \mathrm{mg} / \mathrm{mL}$ 
(Figure 5.15b). The incubation time was then varied while keeping concentrations of Con A and Man2-FSNPs at $1 \mathrm{mg} / \mathrm{mL}$, and $1.5 \mathrm{mg} / \mathrm{mL}$, respectively. As shown in Figure $5.15 \mathrm{c}$, the intensity increased until $75 \mathrm{~min}$ and reached saturation after 2 hours. The incubated lectin spots were characterized by AFM. Images show that the spot was fairly uniformly covered with FSNPs (Figure 5.16). More than single layer of FSNPs was observed in some areas, most likely due to nanoparticle agglomeration before/during incubation. In a control experiment where a non-binding lectin, SBA, was treated with Man2-FSNPs, the signals remained low until after 2 hours, and increased afterwards because of the non-specific adsorption (Figure 5.15c). To minimize particle agglomeration and non-specific adsorption, the glyco-FSNP concentration and the incubation time were kept at $1.5 \mathrm{mg} / \mathrm{mL}$ and 2 hours, respectively, for subsequent studies. 

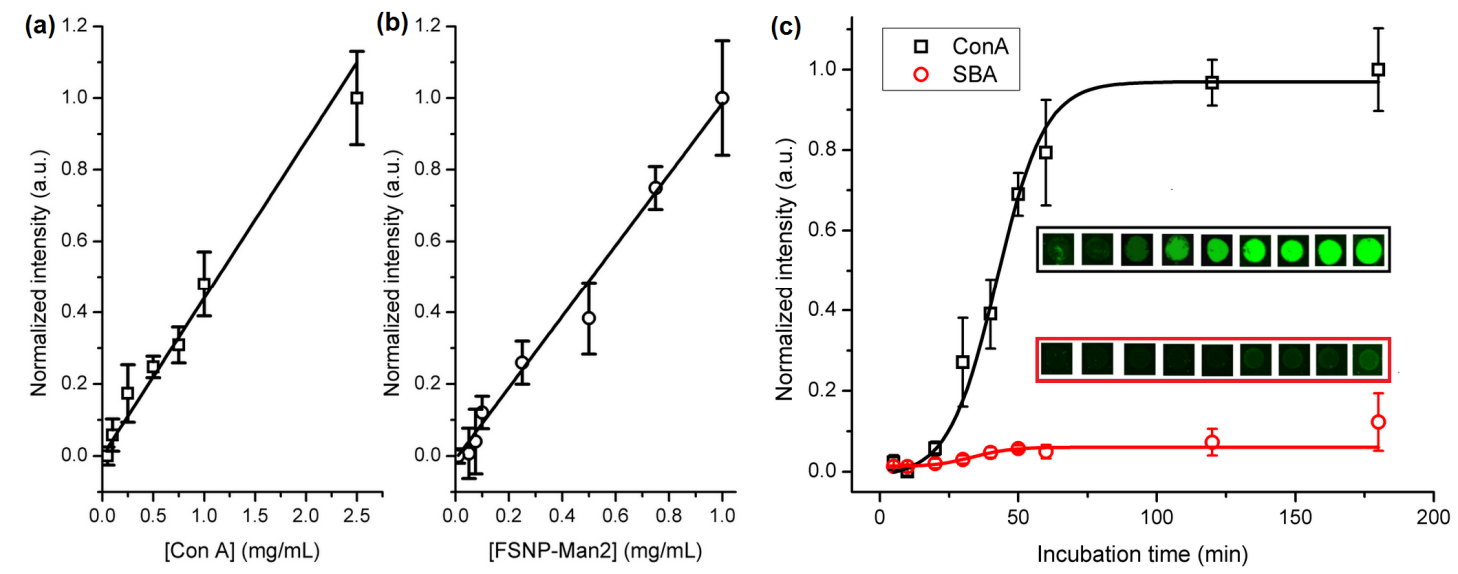

Figure 5.15 Interactions of Man2-FSNPs with super-microarray: (a) fluorescence intensity vs. the printing concentration of Con A ( $\square$ ); (b) fluorescence intensity vs. the concentration of Man2 -FSNPs (O); (c) fluorescence intensity vs. Man2-FSNPs (1.5 $\mathrm{mg} / \mathrm{mL}$ ) incubation time (inserts: fluorescence images of Con A spots (top panel) and SBA spots (bottom panel)). The concentration of Man2-FSNPs in (a) was $1.5 \mathrm{mg} / \mathrm{mL}$, and the printing concentration of Con A was $1 \mathrm{mg} / \mathrm{mL}$ in (b). Each data point was the average of five duplicate spots on the array. The lines were drawn to aid visualization

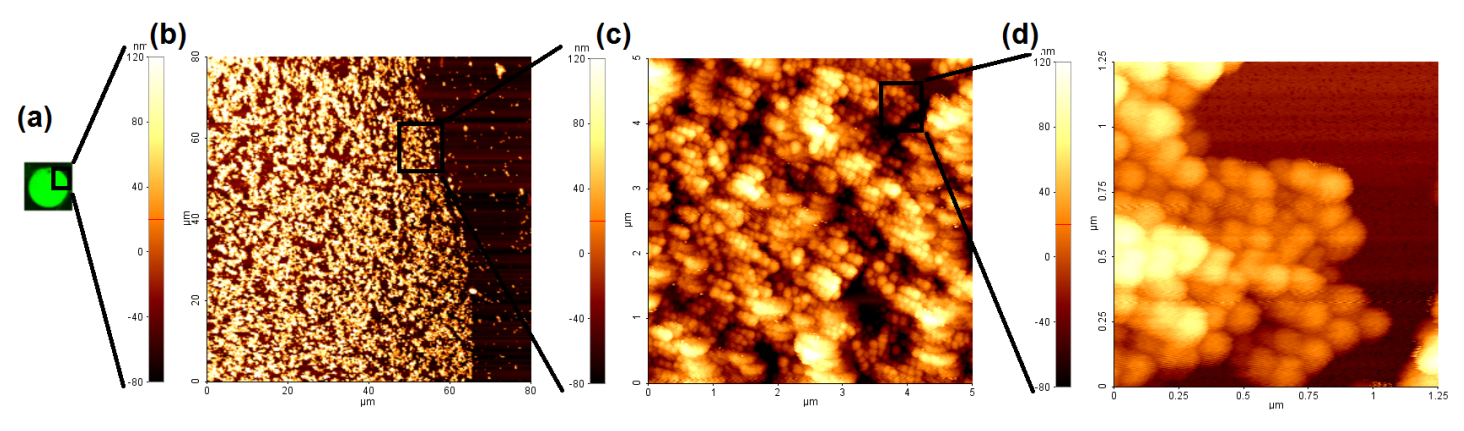

Figure 5.16 Fluorescence (a) and AFM images (b-d) of a printed Con A spot (1 $\mathrm{mg} / \mathrm{mL})$ after incubation with Man2-FSNP $(1.5 \mathrm{mg} / \mathrm{mL})$ for $2 \mathrm{~h}$. 


\section{Super-microarrays for high-throughput ligand screening}

In this study, we evaluate the application of lectin super-microarrays in screening carbohydrate ligands using glyco-FSNPs. A total of 14 lectins were used; their binding carbohydrate ligands are listed in Table 5.3. The lectin microarrays were fabricated following the same protocols and parameters developed in the section described above. Due to the size limitation of the PDMD isolator and printing spot size, only 7 lectins could fit in one isolator well, and therefore, the 14 lectins were divided into two groups. The lectins were printed on GPTMS-functionalized glass slides in 7 x 5 array, and the array was repeated in $2 \times 8$ array format to create 16 microarrays on each chip (Figure 5.13). Figure 5.17 lists the carbohydrate ligands used in this study. Each ligand was coupled on FSNPs following the protocol described above, and the surface density was similar to what was reported in our previous study. ${ }^{166}$ In the binding experiments, a 2 × 8 PDMS isolator was placed on the lectin super-microarray slide to create 16 wells, and different glyco-FSNPs were incubated in each individual well containing the $7 \times 5$ lectin microarray. 
Table 5.3 Lectins and their corresponding carbohydrate binding ligands.

\begin{tabular}{|c|c|c|c|}
\hline Lectin & & Origin & Binding ligands \\
\hline CVN-Q & Cyanovirin- $\mathrm{N} \mathrm{Q}^{50 \mathrm{C}}$ & Cyanobacteria & $\alpha-1,2-$ Man \\
\hline CVN-M & Cyanovirin-N Mut ${ }^{\mathrm{DB}}$ & Cyanobacteria & $\alpha-1,2-\operatorname{Man}$ \\
\hline SBA & Soybean Agglutinin & $\begin{array}{l}\text { Glycine max } \\
\text { (soybean) }\end{array}$ & GalNAc \\
\hline BSA & Bovine serum albumin & Bovine serum & N/A \\
\hline Con A & Concanavalin A & Canavalia ensiformis & Man \\
\hline PNA & Peanut Agglutinin & $\begin{array}{l}\text { Arachis hypogaea } \\
\text { (peanut) }\end{array}$ & $\begin{array}{l}\operatorname{Gal}(\beta-1,3) \\
\text { GalNAc /Gal }\end{array}$ \\
\hline BS-I & $\begin{array}{l}\text { Bandeiraea } \\
\text { simplicifolia }\end{array}$ & $\begin{array}{l}\text { Griffonia } \\
\text { (Bandeiraea) } \\
\text { simplicifolia seeds }\end{array}$ & GalNAc, and Gal \\
\hline OAA & $\begin{array}{l}\text { Oscillatoria Agardhii } \\
\text { Agglutinin }\end{array}$ & Cyanobacteria & $\alpha-1,6-$ Man \\
\hline PFA & Homolog of OAA & $\mathrm{N} / \mathrm{A}$ & $\alpha-1,6-$ Man \\
\hline W77 & Mutant of OAA & Cyanobacteria & $\alpha-1,6-$ Man \\
\hline $\mathrm{DBA}$ & $\begin{array}{l}\text { Dolichos Biflorus } \\
\text { Agglutinin }\end{array}$ & $\begin{array}{l}\text { Dolichos biflorus } \\
\text { (horse gram) seeds }\end{array}$ & GalNAc \\
\hline UEA & $\begin{array}{l}\text { Ulex Europaeus } \\
\text { Agglutinin I }\end{array}$ & $\begin{array}{l}\text { Ulex europaeus } \\
\text { (Furze gorse) seed }\end{array}$ & L-fucose \\
\hline WGA & $\begin{array}{l}\text { Wheat Germ } \\
\text { Agglutinin }\end{array}$ & $\begin{array}{l}\text { Triticum vulgaris } \\
\text { (wheat germ) }\end{array}$ & GlcNAc \\
\hline $\mathrm{RCA}$ & $\begin{array}{l}\text { Ricinus Communis } \\
\text { Agglutinin I }\end{array}$ & $\begin{array}{l}\text { Ricinus communis } \\
\text { (castor bean) seeds }\end{array}$ & Gal/ GalNAc/ Lac \\
\hline
\end{tabular}




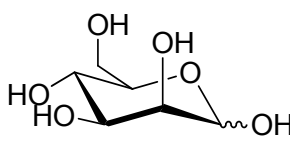

Man

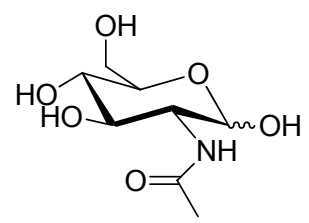

GIcNAc

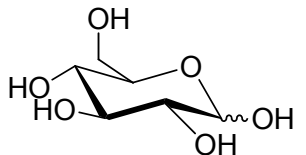

GIc

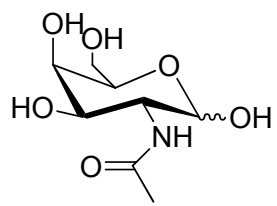

GaINAc

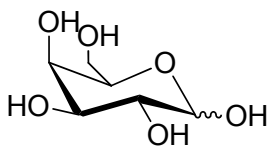

Gal

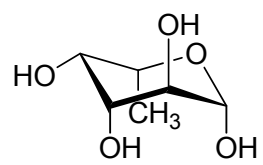

Fuc

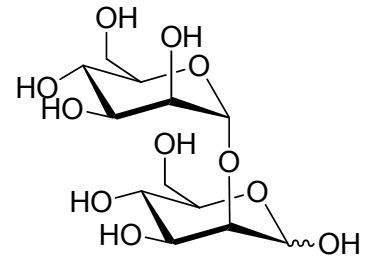

$2 \alpha-M a n 2$

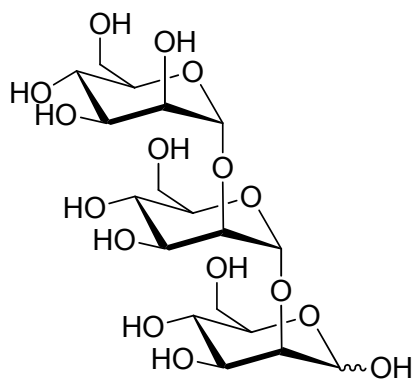

$2 \alpha, 2 \alpha-M a n 3$

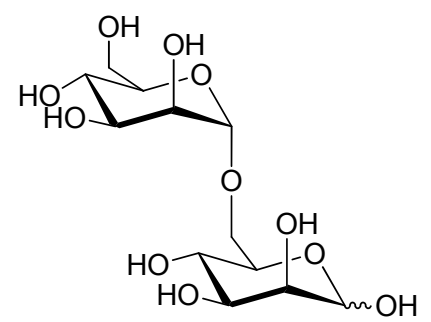

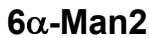

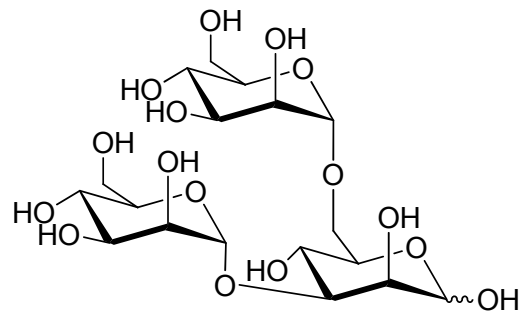

$3 \alpha, 6 \alpha-\operatorname{Man} 3$

Figure 5.17 Carbohydrate structures used in this study.

Results of the microarray analysis are shown in Figure 5.18. The binding pairs such as $2 \alpha-\operatorname{Man} 2 / 2 \alpha, 2 \alpha-\operatorname{Man} 3$ with CVNs/Con A, Man with Con A, Gal with PNA/BS-I, as well as $6 \alpha-M a n 2 / 3 \alpha, 6 \alpha-M a n 3$ with OAA/PFA, Fuc with UEA and Glc with WGA 
show noticeably higher intensities than those non-binding pairs. The results were consistent with the affinity of these glycan-lectin pairs (see association constants shown in Table 5.4), demonstrating the high selectivity of the super-microarrays. The signal intensities also revealed the relative binding affinities of glycan ligands with lectins. For instance, Con A that is a tetrameric protein having saccharides binding sites to $\alpha$-Man and $\alpha$-Glc in the affinity order of $2 \alpha, 2 \alpha-\operatorname{Man} 3\left(3.79 \times 10^{5} \mathrm{M}^{-1}\right)>2 \alpha-$ Man2 $\left(4.17 \times 10^{4} \mathrm{M}^{-1}\right)>\operatorname{Man}\left(8.2 \times 10^{3} \mathrm{M}^{-1}\right)>\operatorname{Glc}\left(1.96 \times 10^{3} \mathrm{M}^{-1}\right)$ (Table 5.4). In this study, Con A spots showed the highest intensity for $2 \alpha, 2 \alpha-\operatorname{Man} 3$ and the lowest for Glc and derivatives (GlcNAc and Lac), and minimal signal was detected for the nonbinding ligand Gal (Figure 5.18). Another group of lectins studied were CV-N mutants $\mathrm{Q}^{50 \mathrm{C}}$ and $\mathrm{Mut}^{\mathrm{DB}}$. These are anti-HIV lectins that recognize $\alpha-1,2$ linked Man structures such as $2 \alpha-\operatorname{Man} 2$ and $2 \alpha, 2 \alpha-\operatorname{Man} 3$ (Table 5.4). The natural ligand is Man9, an oligosaccharide that is difficult and expensive to obtain. ${ }^{184}$ We have shown in Chapter 5.1 that by conjugating the epitope structure of lower Man structures such as $2 \alpha-$ Man2 and $2 \alpha, 2 \alpha-M a n 3$ to gold nanoparticles, the affinity of the resulting glyconanoparticles were increased several orders of magnitude. ${ }^{189}$ These epitope ligands labeled with SFNPs were therefore used in the present studies to probe lectin microarrays. Results in Figure 5.18 shows that $2 \alpha, 2 \alpha$-Man3 conjugated on FSNP exhibited higher fluorescent density than Man2-FSNP, which correlated well with the affinity ranking of the free ligands (Table 5.4) as well as the ITC ${ }^{188}$ and gold GNP ${ }^{189}$ studies. Similar results were also observed for other lectin-glycan binding pairs that the array results were consistent with the affinity rank order of the corresponding free ligands. 
Table 5.4 Association constants $\left(\mathrm{K}_{\mathrm{a}}\right)$ of lectins and glycans.

\begin{tabular}{|c|c|c|}
\hline Lectin & Glycan & $\mathbf{K}_{\mathrm{a}}\left(\mathbf{M}^{-1}\right)$ \\
\hline \multirow{2}{*}{ CVN-Q ${ }^{189}$} & $2 \alpha-\operatorname{Man} 2$ & $\begin{array}{l}1.43 \times 10^{3}(\text { Domain } \mathrm{A}) ; 1.56 \times 10^{4} \\
(\text { Domain B) }\end{array}$ \\
\hline & $2 \alpha, 2 \alpha-\operatorname{Man} 3$ & $\begin{array}{l}2.94 \times 10^{5}(\text { Domain } \mathrm{A}) ; 2.33 \times 10^{4} \\
(\text { Domain B) }\end{array}$ \\
\hline \multirow{2}{*}{$\mathrm{CVN} \mathrm{M}^{188}$} & $2 \alpha-\operatorname{Man} 2$ & $1.32 \times 10^{3}$ \\
\hline & $2 \alpha, 2 \alpha-\operatorname{Man} 3$ & $2.94 \times 10^{5}$ \\
\hline \multirow{4}{*}{ Con $\mathrm{A}^{136}$} & Man & $8.2 \times 10^{3}$ \\
\hline & $2 \alpha-\operatorname{Man} 2$ & $4.17 \times 10^{4}$ \\
\hline & $2 \alpha, 2 \alpha-\operatorname{Man} 3$ & $3.79 \times 10^{5}$ \\
\hline & Glc & $1.96 \times 10^{3}$ \\
\hline $\mathrm{SBA}^{231}$ & GalNAc & $1.51 \times 10^{6}$ \\
\hline \multirow{2}{*}{$\mathrm{PNA}^{232}$} & Gal & $0.98 \times 10^{3}$ \\
\hline & GalNAc & $2.43 \times 10^{3}$ \\
\hline \multirow{2}{*}{ BS-I ${ }^{147}$} & Gal & $2.1 \times 10^{4}$ \\
\hline & GalNAc & $1.87 \times 10^{5}$ \\
\hline $\mathrm{DBA}^{233}$ & GalNAc & $4.2 \times 10^{3}$ \\
\hline $\mathrm{WGA}^{234}$ & GlcNAc & $0.4 \times 10^{3}$ \\
\hline \multirow{2}{*}{$\mathrm{RCA}^{176}$} & Gal & $2.2 \times 10^{3}$ \\
\hline & GalNAc & $4.84 \times 10^{4}$ \\
\hline
\end{tabular}




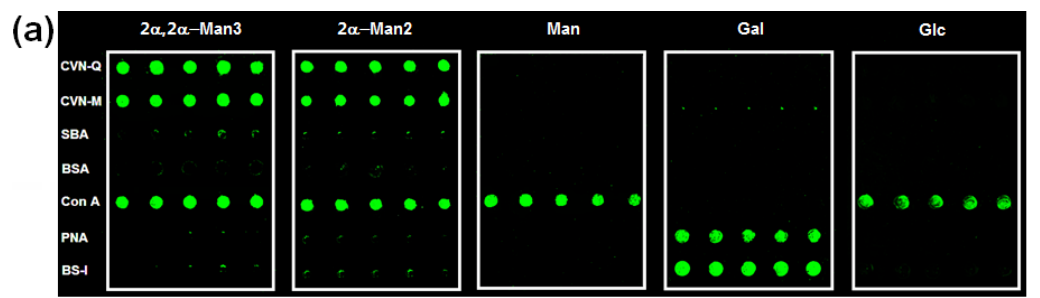

(b)
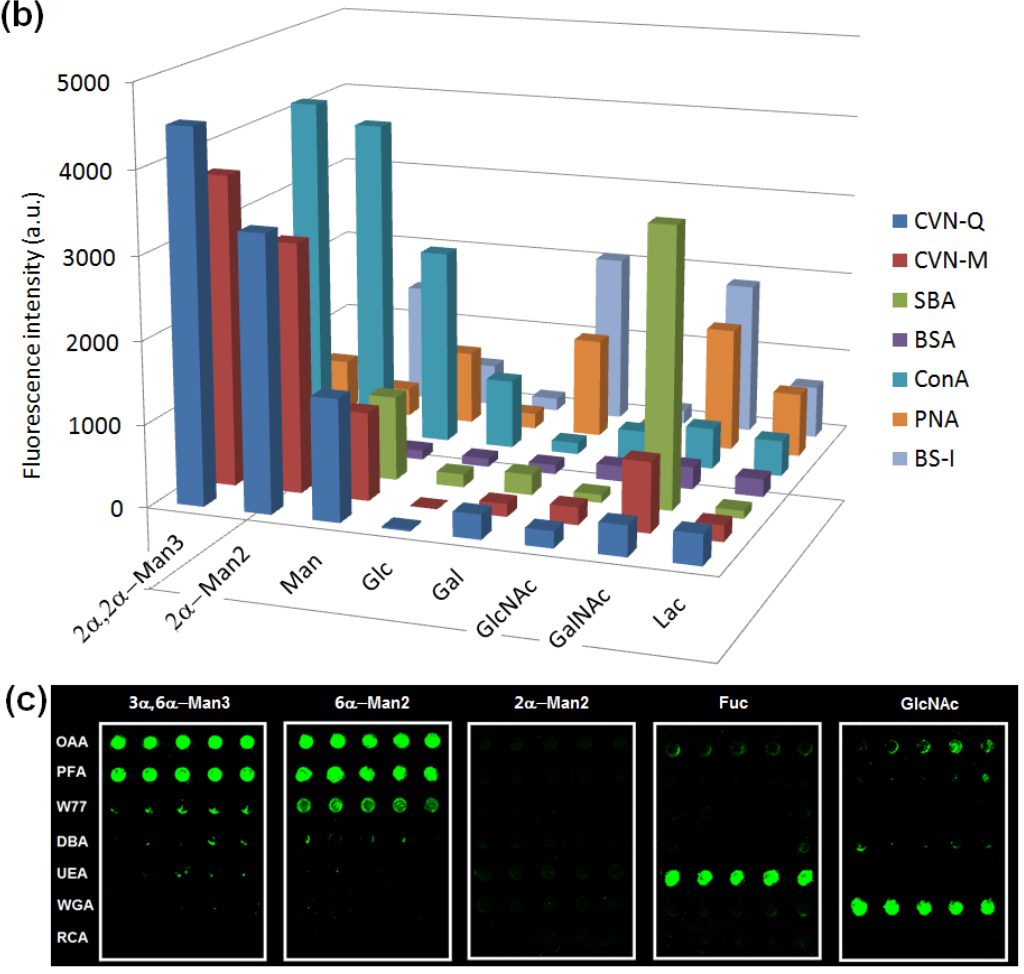

(d)

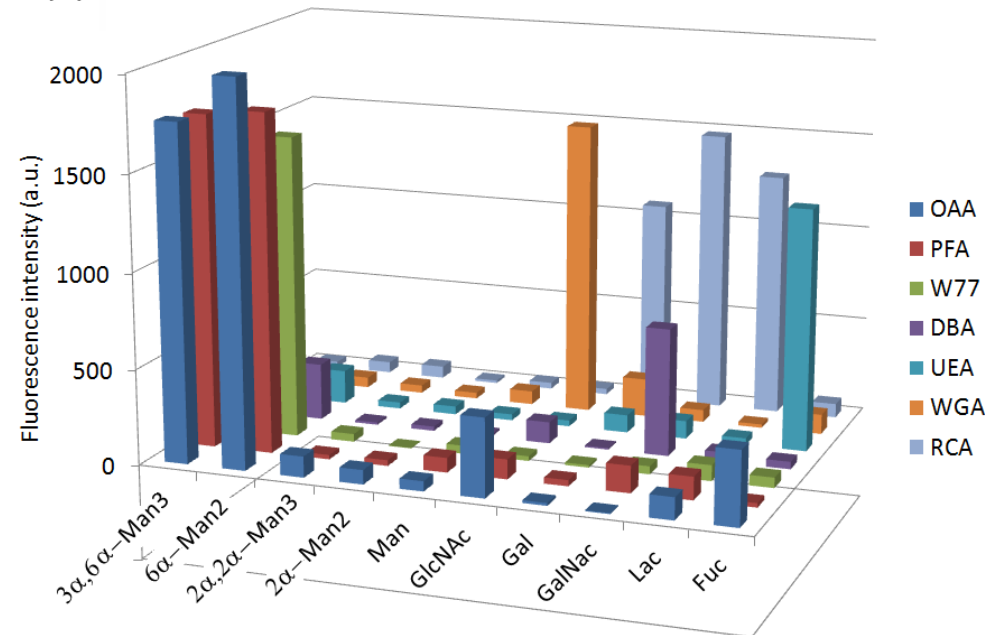

Figure 5.18 Fluorescence image (a, c) and fluorescence intensity (b, d) of lectin supermicroarrays interacting with glyco-FSNPs. 
Super-microarrays for high throughput binding affinity determination

The 2 x 8 PDMS isolator creates 16 individual microarrays, and therefore, 16 different assays can be carried out simultaneously on a single super-microarray. When 16 ligand competition assays are performed, a dose-response curve can be plotted, from which the $\mathrm{IC}_{50}$ value can be derived and the dissociation constant subsequently calculated. This provides a high throughput means to determine binding affinity from a single super-microarray.

Competition binding assays were performed by mixing free competing ligands of varying concentrations with a fixed concentration of glyco-FSNPs and the resulting solutions were transferred to the wells on the super-microarray. Figure 5.19 showed a typical fluorescence image of the lectin super-microarray after incubating with Man2FSNP and varying concentration of $2 \alpha$-Man2. It can be seen that only the binding lectins for 2 $\alpha-\mathrm{Man} 2(\mathrm{CVN}-\mathrm{Q}, \mathrm{CVN}-\mathrm{M}$ and Con A) exhibited strong and different signals with varying concentrations of $2 \alpha-M a n 2$. Further, the fluorescence intensity decreased with increasing $2 \alpha-$ Man2 concentration, which was anticipated since $2 \alpha$ Man2 competed with Man2-FSNP for binding the lectins on the array. By plotting the fluorescence intensity against the concentration of $2 \alpha-M a n 2$, a dose response curve was obtained, from which the $\mathrm{IC}_{50}$ value can be derived. Figure $5.19 \mathrm{~b}-\mathrm{d}$ show the results from the three binding lectins $\mathrm{CVN}-\mathrm{Q}, \mathrm{CVN}-\mathrm{M}$ and Con A. Following a revised Cheng-Prusoff equation

$$
\mathrm{K}_{\mathrm{D}}=\mathrm{IC}_{50} /\left(1+[\mathrm{M}] / \mathrm{K}_{\mathrm{d}}\right)
$$


where $\mathrm{K}_{\mathrm{d}}$ is the dissociation constant of the free ligand with the corresponding lectin, $\mathrm{K}_{\mathrm{D}}$, the apparent dissociation constant of immobilized lectin with glyco-FSNP was calculated, ${ }^{154,206}$ and results are shown in Table 5.5.
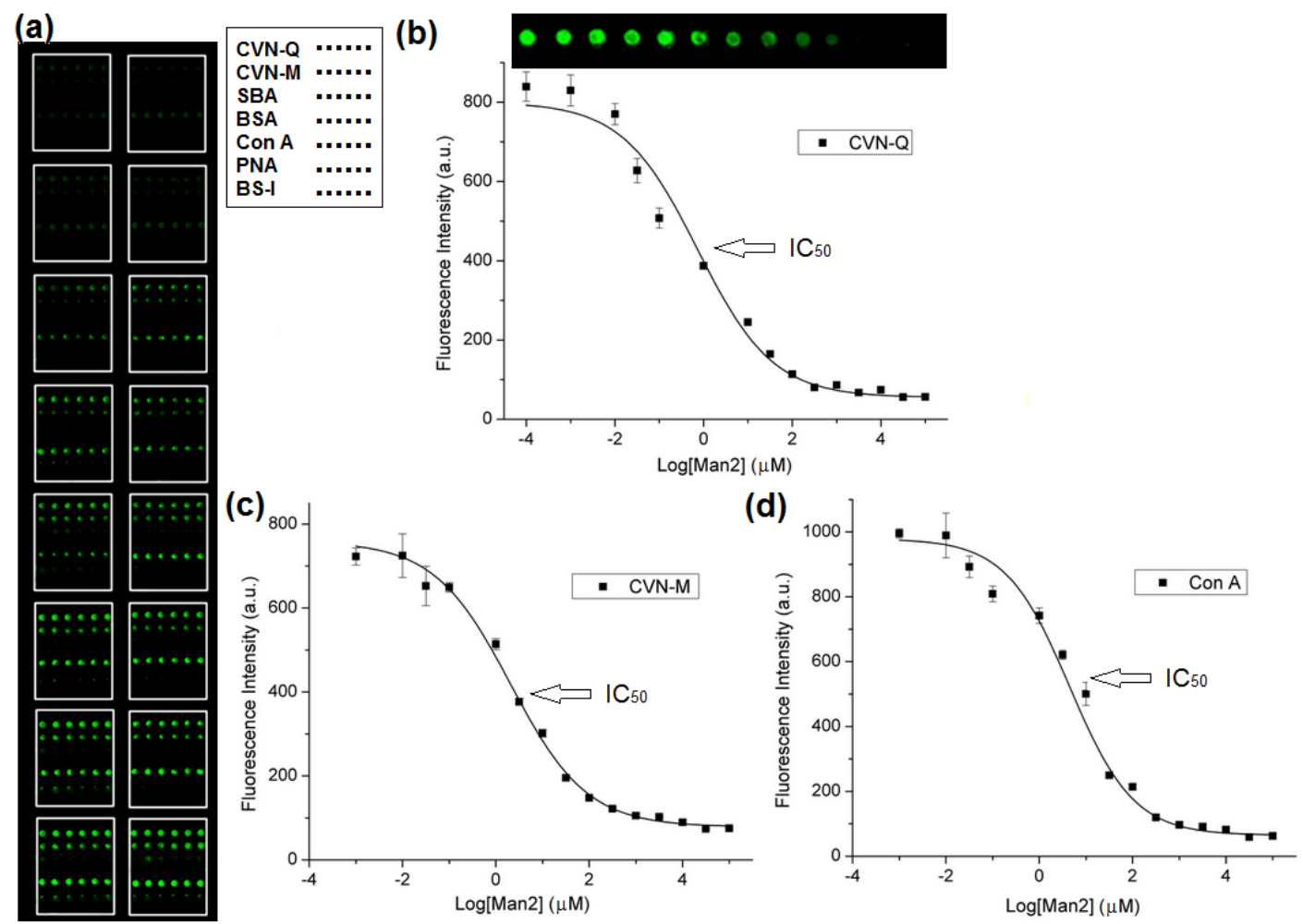

Figure 5.19 (a) Fluorescence image of a lectin super-microarray after incubating with Man2-FSNP and varying concentrations of $2 \alpha-M a n 2$. (b-d) Fluorescence intensities $v s$. free $2 \alpha-M a n 2$ concentration for CVN-Q CVN-M (c) and Con A (d). Insert in (b) shows the fluorescence images of the corresponding spots. 
Table 5.5 Apparent $K_{D}$ values of glyco-FSNPs with lectins obtained from supermicroarrays.

\begin{tabular}{|c|c|c|c|c|c|}
\hline & $\begin{array}{c}\text { Ligand density } \\
(\text { ligand/nm²) }\end{array}$ & CVN-Q & CVN-M & Con A & PNA \\
\hline $\begin{array}{l}\text { FSNP- } \\
\text { Man3 }\end{array}$ & 1.3 & $\begin{array}{c}1.66 \mathrm{nM} \\
(15.5 \mu \mathrm{M})\end{array}$ & $\begin{array}{l}0.43 \mathrm{nM} \\
(3.4 \mu \mathrm{M})\end{array}$ & $\begin{array}{l}0.0083 \mathrm{nM} \\
(2.97 \mu \mathrm{M})\end{array}$ & $\mathrm{N} / \mathrm{D}^{3}$ \\
\hline $\begin{array}{l}\text { FSNP- } \\
\text { Man2 }\end{array}$ & 1.5 & $\begin{array}{c}16.6 \mathrm{nM} \\
(89.8 \mu \mathrm{M})\end{array}$ & $\begin{array}{c}589 \mathrm{nM} \\
(757 \mu \mathrm{M})\end{array}$ & $\begin{array}{l}5.40 \mathrm{nM} \\
(24 \mu \mathrm{M})\end{array}$ & N/D \\
\hline $\begin{array}{l}\text { FSNP- } \\
\text { Man }\end{array}$ & 4.2 & $\mathrm{~N} / \mathrm{D}$ & $\mathrm{N} / \mathrm{D}$ & $\begin{array}{l}13.7 \mathrm{nM} \\
(470 \mu \mathrm{M})\end{array}$ & N/D \\
\hline $\begin{array}{c}\text { FSNP- } \\
\text { Gal }\end{array}$ & 4.3 & $\mathrm{~N} / \mathrm{D}$ & $\mathrm{N} / \mathrm{D}$ & $\mathrm{N} / \mathrm{D}$ & $\begin{array}{c}114 \mathrm{nM} \\
(1050 \mu \mathrm{M})\end{array}$ \\
\hline
\end{tabular}

${ }^{1}$ Ligand density was measured by anthrone- $\mathrm{H}_{2} \mathrm{SO}_{4}$ colorimetric method.

${ }^{2}$ The data in brackets are dissociation constants of the free ligand with the lectin. ${ }^{3} \mathrm{~N} / \mathrm{D}=$ not detectable.

A dramatic increase in affinity was observed when glycans attached on nanoparticle surface. Results showed that the multivalent presentation of carbohydrate ligands significantly enhanced the binding affinity of FSNPs by $4 \sim 6$ orders of magnitude in comparison to the free ligands in solution. In addition, the apparent affinity of FSNPGal with its binding lectin PNA was determined as well, and similarly $\mathrm{K}_{\mathrm{D}}$ was 7 orders of magnitude lower than $K_{d}$ of free galactose ligand. The apparent $K_{D}$ values (Table 5.5) were comparable to those obtained using other microarray platforms, such as surface plasmon resonance ${ }^{235,236}$ and evanescent-field fluorescence. ${ }^{213}$ 


\section{Conclusion}

A new platform has been developed to study glycan-lectin interactions. It is based on lectin microarrays which are probed with glycans labeled with dye-doped nanoparticles. This labeling technique has several key advantages. First, the coupling chemistry is highly general and can be applied to a wide range of glycans including higher glycan structures, underivatized carbohydrates, as well as reducing sugars. ${ }^{237}$ Secondly, compared with fluorescent dyes, dye-doped nanoparticles provide higher fluorescence intensity and much improved photo-stability. The labeled glycans can be readily purified by dialysis thus avoiding the potential complex solution-phase purification procedure when fluorescent dyes are used. Thirdly, multiple copies of the glycan can be conjugated on one nanoparticle introducing multivalency effect that significantly enhances their interactions with lectins.

The lectin super-microarrays further increase the throughput of microarrays by creating multiple microarrays on the same chip. Thus different assays can be performed simultaneously permitting large-scale glycan profiling. Furthermore, ligand competition assays can be conducted on a single super-microarray to afford the dose response curve, from which the $\mathrm{IC}_{50}$ and apparent $\mathrm{K}_{\mathrm{D}}$ values can be readily obtained. This represents a new analytical method for affinity measurement. Compared with the reported methods of nuclear magnetic resonance (NMR), ${ }^{238}$ enzyme-linked immunosorbent assay (ELISA), ${ }^{239}$ isothermal titration calorimetry (ITC), ${ }^{240}$ precipitation inhibition, ${ }^{241}$ equilibrium dialysis, ${ }^{242}$ spectrophotometry, ${ }^{243}$ fluorescence 
competition assay, ${ }^{155}$ surface plasmon resonance (SPR), ${ }^{236,244}$ carbohydrate microarrays, ${ }^{204}$ and quartz-crystal microbalance (QCM) ${ }^{245,246}$ our method allows for high-throughput data outputs and much reduced glycan consumption (only $\sim 3 \mu \mathrm{g}$ of glycans consumed for each assay). The high throughput nature of lectin supermicroarrays in conjunction with the versatility of nanoparticle-based glycan labeling technique provides a robust and unique approach ideally suited for quantitative glycan profiling in a large library. The protocol could also be applied to other biological entities such as DNA, antibodies, and cells. The technology presented herein thus opens new opportunities facilitating the development of glycomics as well as application in disease diagnosis and therapeutics. 


\section{Chapter 6. Conclusion}

Merging nanotechnology with biology has seen exponential growth of research activities in functional bio-nanomaterials. Nanomaterials in general, and glyconanoparticles in particular, are witnessing a tremendous interest for a wide range of applications in chemistry, biology and medicine, and their merits are well known in comparison to other formats. Essential to glyconanoparticle preparation is the conjugation chemistry that can efficiently attach carbohydrates to the nanomaterials. We developed a simple and versatile photocoupling chemistry that allows the covalent immobilization of a wide range of carbohydrate structures to nanomaterials, including gold and fluorescent silica nanoparticles. The coupling reaction was efficient and high yielding, and the resulting surface-bound carbohydrate ligands retained their binding affinities and selectivities. The development of new analytical methods to characterize glyconanoparticle-protein interactions, including fluorescence competition assays, dynamic light scattering and isothermal calorimetry, highlight the importance of quantitative analysis of structural and functional properties of glyconanomaterials, especially the biorecognition properties that must be carefully analyzed in the context of ligand presentation and display. Results from my studies have shown that glyconanoparticles are highly potent - potentially superior - vehicles for targeting carbohydrate-mediated biological effects due to the multivalency effects commonly associated with carbohydrate-protein interactions. Nanoparticles as carriers can greatly enhance the binding affinity of the carbohydrate ligands, and in addition, the affinity is 
significantly impacted by the carbohydrate presentation on nanoparticle surfaces, which could be applied for enhanced recognition of biologically significant lectins, such as anti-HIV CV-N in our study. The new glycan label, dye-doped silica nanoparticles, greatly facilitated the high-throughput labeling using nanomaterials as the scaffold, and FSNP-labeled glycans have shown potential applications in protein recognition, bacteria imaging, and high-throughput glycan screening on lectin microarrays. With the wealth of existing results on glyconanomaterials and their demonstrated multivalent interactions with proteins, advanced developments in therapeutic and diagnostic applications based on biologically-functionalized nanomaterials are poised to evolve. 


\section{References}

(1) Koch, C. C. Nanostructured Materials: Processing Properties, and Potential Applications; Noyes Publications: Norwich, NY, 2002.

(2) Gonsalves, K.; Halberstadt, C.; Laurencin, C. T.; Nair, L. Biomedical

Nanostructures John Wiley \& Sons, Inc.: Hoboken, NJ, 2007.

(3) Rosi, N. L.; Mirkin, C. A. Chem. Rev. 2005, 105, 1547-1562.

(4) Yang, H.; Xia, Y. Adv. Mater. 2007, 19, 3085-3087.

(5) Moran, N. Nat Biotech 2006, 24, 121-121.

(6) Kataoka, K.; Scholz, C.; Editors Polymers in Biomedical Applications, 2007; Vol. $32(8-9)$.

(7) Sperling, R.; Rivera, G. P.; Zhang, F.; Zanella, M.; Parak, W. Chem. Soc. Rev. 2008, 37, 1896-1908.

(8) Wilson, R. Chem. Soc. Rev. 2008, 37, 2028-2045.

(9) Liu, R.; Liew, R.; Zhou, J.; Xing, B. Angew. Chem. Int. Ed. 2007, 46, 8799-8803.

(10) Elghanian, R.; Storhoff, J. J.; Mucic, R. C.; Letsinger, R. L.; Mirkin, C. A.

Science 1997, 277, 1078 - 1081.

(11) Lin, C.-C.; Yeh, Y.-C.; Yang, C.-Y.; Chen, G.-F.; Chen, Y.-C.; Wu, Y.-C.; Chen, C.-C. Chem. Commun. 2003, 23, 2920-2921.

(12) Bowman, M.-C.; Ballard, T. E.; Ackerson, C. J.; Feldheim, D. L.; Margolis, D. M.; Melander, C. J. Am. Chem. Soc. 2008, 130, 6896-6897.

(13) Hu, J.; Li, L.-s.; Yang, W.; Manna, L.; Wang, L.-w.; Alivisatos, A. P. Science 2001, 292, 2060-2063.

(14) Nirmal, M.; Brus, L. Acc. Chem. Res. 1999, 32, 407-414.

(15) Bruchez, M., Jr.; Moronne, M.; Gin, P.; Weiss, S.; Alivisatos, A. P. Science 1998, 281, 2013-2016.

(16) Lu, A. H.; Salabas, E. L.; Schuth, F. Angew. Chem. Int. Ed. 2007, 46, 1222-1244.

(17) Gupta, A. K.; Gupta, M. Biomaterials 2005, 26, 3995-4021.

(18) Laurent, S.; Forge, D.; Port, M.; Roch, A.; Robic, C.; Elst, L. V.; Muller, R. N. Chem. Rev. 2008, 108, 2064-2110. 
(19) Tartaj, P.; Morales, M. D.; Veintemillas-Verdaguer, S.; Gonzalez-Carreno, T.; Serna, C. J. J. Phys. D: Appl. Phys. 2003, 36, R182-R197.

(20) Pankhurst, Q. A.; Connolly, J.; Jones, S. K.; Dobson, J. J. Phys. D: Appl. Phys. 2003, 36, R167-R181.

(21) Berry, C. C.; Curtis, A. S. G. J. Phys. D: Appl. Phys. 2003, 36, R198-R206.

(22) Jun, Y. W.; Seo, J. W.; Cheon, A. Acc. Chem. Res. 2008, 41, 179-189.

(23) Ito, A.; Shinkai, M.; Honda, H.; Kobayashi, T. J. Biosci. Bioeng. 2005, 100, 1-11.

(24) Gupta, A. K.; Naregalkar, R. R.; Vaidya, V. D.; Gupta, M. Nanomedicine 2007, 2, 23-39.

(25) Bonnemain, B. J. Drug Targeting 1998, 6, 167-174.

(26) Martina, M. S.; Fortin, J. P.; Menager, C.; Clement, O.; Barratt, G.; GrabielleMadelmont, C.; Gazeau, F.; Cabuil, V.; Lesieur, S. J. Am. Chem. Soc. 2005, 127, 10676-10685.

(27) Hacliipanayis, C. G.; Bonder, M. J.; Balakrishanan, S.; Wang, X.; Mao, H.; Hadjipanayis, G. C. Small 2008, 4, 1925-1929.

(28) Giri, S.; Trewyn, B. G.; Stellmaker, M. P.; Lin, V. S. Y. Angew. Chem. Int. Ed. 2005, 44, 5038-5044.

(29) Yoon, T. J.; Kim, J. S.; Kim, B. G.; Yu, K. N.; Cho, M. H.; Lee, J. K. Angew. Chem. Int. Ed. 2005, 44, 1068-1071.

(30) Dobson, J. Drug Dev. Res. 2006, 67, 55-60.

(31) Polyak, B.; Friedman, G. Expert Opinion on Drug Delivery 2009, 6, 53-70.

(32) Jordan, A.; Scholz, R.; Wust, P.; Fahling, H.; Felix, R.; Elsevier Science Bv: 1999, p 413-419.

(33) Johannsen, M.; Gneveckow, U.; Eckelt, L.; Feussner, A.; Waldofner, N.; Scholz, R.; Deger, S.; Wust, P.; Loening, S. A.; Jordan, A. International Journal of Hyperthermia 2005, 21, 637-647.

(34) Ito, A.; Shinkai, M.; Honda, H.; Kobayashi, T. Cancer Gene Ther. 2001, 8, 649654.

(35) Liberti, P. A.; Rao, C. G.; Terstappen, L.; Elsevier Science Bv: 2001, p 301-307. 
(36) Morisada, S.; Miyata, N.; Iwahori, K. J. Microbiol. Methods 2002, 51, 141-148.

(37) El-Boubbou, K.; Gruden, C.; Huang, X. J. Am. Chem. Soc. 2007, 129, 1339213397.

(38) Xu, C. J.; Xu, K. M.; Gu, H. W.; Zheng, R. K.; Liu, H.; Zhang, X. X.; Guo, Z. H.;

Xu, B. J. Am. Chem. Soc. 2004, 126, 9938-9939.

(39) Sasaki, T.; Iwasaki, N.; Kohno, K.; Kishimoto, M.; Majima, T.; Nishimura, S. I.;

Minami, A. J. Biomed. Mater. Res., Part A 2008, 86A, 969-978.

(40) Jun, Y. W.; Lee, J. H.; Cheon, J. Angew. Chem. Int. Ed. 2008, 47, 5122-5135.

(41) Josephson, L.; Tung, C. H.; Moore, A.; Weissleder, R. Bioconjugate Chem. 1999, $10,186-191$.

(42) Wise, F. W. Acc. Chem. Res. 2000, 33, 773-780.

(43) Labhasetwar, V.; Leslie-Pelecky, D. L. Biomedical applications of nanotechnology; John Wiley \& Sons, Inc.: Hoboken, NY, 2007.

(44) Papazoglou, E. S.; Parthasarathy, A. BioNanotechnology; Morgan \& Claypool: San Francisco, 2007.

(45) Star, A.; Gabriel, J. C. P.; Bradley, K.; Gruner, G. Nano Lett. 2003, 3, 459-463.

(46) Dahl, J. A.; Maddux, B. L. S.; Hutchison, J. E. Chem. Rev. 2007, 107, 2228-2269.

(47) Ratner, D. M.; Adams, E. W.; Su, J.; O'Keefe, B. R.; Mrksich, M.; Seeberger, P. H. ChemBioChem 2004, 5, 379-382.

(48) Ratner, D. M.; Seeberger, P. H. Curr. Pharm. Des. 2007, 13, 173-183.

(49) Lee, M. R.; Shin, I. Angew. Chem. Int. Ed. 2005, 44, 2881-2884.

(50) Park, S.; Shin, I. Org. Lett. 2007, 9, 1675-1678.

(51) Liang, P. H.; Wang, S. K.; Wong, C. H. J. Am. Chem. Soc. 2007, 129, $11177-$ 11184.

(52) Fazio, F.; Bryan, M. C.; Blixt, O.; Paulson, J. C.; Wong, C. H. J. Am. Chem. Soc. 2002, 124, 14397-14402.

(53) Sun, X. L.; Stabler, C. L.; Cazalis, C. S.; Chaikof, E. L. Bioconjugate Chem. 2006, $17,52-57$. 
(54) Xia, B. Y.; Kawar, Z. S.; Ju, T. Z.; Alvarez, R. A.; Sachdev, G. P.; Cummings, R. D. Nat. Methods 2005, 2, 845-850.

(55) Park, S.; Lee, M. R.; Shin, I. Bioconjugate Chem. 2009, 20, 155-162.

(56) Zhi, Z.-1.; Powell, A. K.; Turnbull, J. E. Anal. Chem. 2006, 78, 4786-4793.

(57) Liu, F.-T.; Rabinovich, G. A. Nat. Rev. Cancer 2005, 5, 29-41.

(58) Dube, D. H.; Bertozzi, C. R. Nat. Rev. Drug. Discov. 2005, 4, 477-488.

(59) Fuster, M. M.; Esko, J. D. Nat. Rev. Cancer 2005, 5, 526-542.

(60) Szymanski, C. M.; Wren, B. W. Nat. Rev. Microbiol. 2005, 3, 225-237.

(61) Ohtsubo, K.; Marth, J. D. Cell 2006, 126, 855-867.

(62) Sharon, N. Biochim. Biophys. Acta, Gen. Subj. 2006, 1760, 527-537.

(63) Crocker, P. R.; Paulson, J. C.; Varki, A. Nat. Rev. Immunol. 2007, 7, 255-266.

(64) Blow, N. Nature 2009, 457, 617-620.

(65) Sears, P.; Wong, C.-H. Science 2001, 291, 2344-2350.

(66) Koeller, K. M.; Wong, C.-H. Nature 2001, 409, 232-240.

(67) Seeberger, P. H. Chem. Soc. Rev. 2008, 37, 19-28.

(68) Lee, Y. C.; Lee, R. T. Acc. Chem. Res. 1995, 28, 321-327.

(69) Mammen, M.; Choi, S. K.; Whitesides, G. M. Angew. Chem. Int. Ed. 1998, 37, 2754-2794.

(70) J. Huskens Curr. Opin. Chem. Biol. 2006, 10, 537-543.

(71) U. Drechsler; B. Erdogan; V. M. Rotello Chem. Eur. J. 2004, 10, 5570-5579.

(72) Ernst, B.; Magnani, J. L. Nat. Rev. Drug. Discov. 2009, 8, 661-677.

(73) Wang, D. Proteomics 2003, 3, 2167-2175.

(74) Feizi, T.; Chai, W. Nat. Rev. Mol. Cell. Biol. 2004, 5, 582-588.

(75) Stevens, J.; Blixt, O.; Paulson, J. C.; Wilson, I. A. Nat. Rev. Microbiol. 2006, 4, 857-864.

(76) Coullerez, G.; Seeberger, P. H.; Textor, M. Macromol. Biosci. 2006, 6, 634-647.

(77) Laurent, N.; Voglmeir, J.; Flitsch, S. L. Chem. Commun. 2008, 4400-4412.

(78) Park, S.; Lee, M.-R.; Shin, I. Chem. Soc. Rev. 2008, 37, 1579-1591.

(79) Horlacher, T.; Seeberger, P. H. Chem. Soc. Rev. 2008, 37, 1414-1422. 
(80) Liang, P.-H.; Wu, C.-Y.; Greenberg, W. A.; Wong, C.-H. Curr. Opin. Chem. Biol. 2008, $12,86-92$.

(81) Jaiswal, J. K.; Mattoussi, H.; Mauro, J. M.; Simon, S. M. Nat. Biotechnol. 2003, $21,47-51$.

(82) Rojo, J.; Díaz, V.; de la Fuente, J. M.; Segura, I.; Barrientos, A. G.; Riese, H. H.; Bernade, A.; Penadés, S. ChemBioChem 2004, 5, 291-297.

(83) Sihelnikova, L.; Tvaroska, I. Chem. Pap. 2007, 61, 237-255.

(84) De, M.; Ghosh, P. S.; Rotello, V. M. Adv. Mater. 2008, 20, 4225-4241.

(85) Cipolla, L.; Peri, F.; Airoldi, C. Anti-Cancer Agents Med. Chem. 2008, 8, 92-121.

(86) Huang, H.; Yuan, Q.; Yang, X. Colloids Surf., B 2004, 39, 31-37.

(87) Chen, Y.; Ji, T.; Rosenzweig, Z. Nano Lett. 2003, 3, 581-584.

(88) El-Kouedi, M.; Keating, C. D. In Nanobiotechnology; Niemeyer, C. M., Mirkin, C. A., Eds.; Wiley-VCH Weinheim, 2004.

(89) Brust, M.; Walker, M.; Bethell, D.; Schiffrin, D. J.; Whyman, R. J. Chem. Soc., Chem. Commun. 1994, 801-802.

(90) Niemeyer, C. M. Angew. Chem. Int. Ed. 2001, 40, 4128-4158.

(91) Daniel, M.-C.; Astruc, D. Chem. Rev. 2003, 104, 293-346.

(92) Rojas, T. C.; de la Fuente, J. M.; Barrientos, A. G.; Penadés, S.; Ponsonnet, L.; Fernández, A. Adv. Mater. 2002, 14, 585-588.

(93) M. J. Hernáiz; J. M. de la Fuente; Á. G. Barrientos; S. Penadés Angew. Chem. Int. Ed. 2002, 41, 1554-1557.

(94) de la Fuente, J. M.; Eaton, P.; Barrientos, A. G.; Menendez, M.; Penadés, S. J. Am. Chem. Soc. 2005, 127, 6192-6197.

(95) de Paz, J. L.; Ojeda, R.; Barrientos, A. G.; Penadés, S.; Martin-Lomas, M.

Tetrahedron: Asymmetry 2005, 16, 149-158.

(96) Zhang, J.; Geddes, C. D.; Lakowicz, J. R. Anal. Biochem. 2004, 332, 253-260.

(97) Koen, M. H.; Adriana Carvalho de, S.; Maljaars, C. E. P.; Gerrit, J. G.; Johannis, P. K. Eur. J. Org. Chem. 2005, 2005, 3650-3659.

(98) Schofield, C. L.; Field, R. A.; Russell, D. A. Anal. Chem. 2007, 79, 1356-1361. 
(99) Huang, C. C.; Chen, C. T.; Shiang, Y. C.; Lin, Z. H.; Chang, H. T. Anal. Chem. 2009, $81,875-882$.

(100) Marradi, M.; Alcántara, D.; de la Fuente, J. M.; García-Martín, M. L.; Cerdán, S.; Penadés, S. Chem. Commun. 2009, 3922-3924.

(101) Robinson, A.; Fang, J. M.; Chou, P. T.; Liao, K. W.; Chu, R. M.; Lee, S. J.

ChemBioChem 2005, 6, 1899-1905.

(102) Babu, P.; Sinha, S.; Surolia, A. Bioconjugate Chem. 2007, 18, 146-151.

(103) Svarovsky, S. A.; Barchi, J. J. J. In Frontiers in Modern Carbohydrate

Chemistry; Demchenko, A. V., Ed.; Oxford University Press: Oxford, UK, 2007.

(104) Earhart, C.; Jana, N. R.; Erathodiyil, N.; Ying, J. Y. Langmuir 2008, 24, 62156219.

(105) Otsuka, H.; Akiyama, Y.; Nagasaki, Y.; Kataoka, K. J. Am. Chem. Soc. 2001, 123, 8226-8230.

(106) Liu, L.-H.; Yan, M. Acc. Chem. Res. 2010, 43, 1434-1443.

(107) Homola, J. In Springer Series on Chemical Sensors and Biosensors Urban, G., Ed.; Springer-Verlag GmbH: Heidelberg, 2006; Vol. 4.

(108) Oh, E.; Lee, D.; Kim, Y.-P.; Cha, S. Y.; Oh, D.-B.; Kang, H. A.; Kim, J.; Kim, H.-S. Angew. Chem. Int. Ed. 2006, 45, 7959-7963.

(109) Storhoff, J. J.; Lucas, A. D.; Garimella, V.; Bao, Y. P.; Müller, U. R. Nat.

Biotechnol 2004, 22, 883-887.

(110) Sun, Y.; Xia, Y. Analyst 2003, 128, 686-691.

(111) Jiang, Y.; Zhao, H.; Zhu, N.; Lin, Y.; Yu, P.; Mao, L. Angew. Chem. Int. Ed.

2008, 47, 8601-8604.

(112) Kamat, P. V. J. Phys. Chem. B 2002, 106, 7729 -7744.

(113) Okamoto, T.; Yamaguchi, I. J. Phys. Chem. B 2003, 107, 10321-10324.

(114) Lis, H.; Sharon, N. Chem. Rev. 1998, 98, 637-674.

(115) Ngundi, M. M.; Kulagina, N. V.; Anderson, G. P.; Taitt, C. R. Exp. Rev.

Proteomics 2006, 3, 511-524. 
(116) Jesus M. de la Fuente; Barrientos, A. G.; Rojas, T. C.; Rojo, J.; Canada, J.;

Fernandez, A.; Penades, S. Angew. Chem. Int. Ed. 2001, 40, 2257-2261.

(117) Maria, J. H.; Jesus, M. d. 1. F.; Africa, G. B.; Soledad, P. Angew. Chem. Int. Ed. 2002, 41, 1554-1557.

(118) Kim, Y.-P.; Park, S.; Oh, E.; Oh, Y.-H.; Kim, H.-S. Biosens. Bioelectron. 2009, 24, 1189-1194.

(119) Ojeda, R.; de Paz, J. L.; Barrientos, A. G.; Martin-Lomas, M.; Penades, S.

Carbohydrate Res. 2007, 342, 448-459.

(120) Mikkel, T.; Jorgen, S.; Knud, J. Chem. Eur. J. 2009, 15, 1649-1660.

(121) Lee, M.-R.; Shin, I. Org. Lett. 2005, 7, 4269-4272.

(122) Seo, J. H.; Adachi, K.; Lee, B. K.; Kang, D. G.; Kim, Y. K.; Kim, K. R.; Lee, H.

Y.; Kawai, T.; Cha, H. J. Bioconjugate Chem. 2007, 18, 2197-2201.

(123) Yan, M.; Ren, J. Chem. Mater. 2004, 16, 1627-1632.

(124) Liu, L.; Engelhard, M. H.; Yan, M. J. Am. Chem. Soc. 2006, 128, 14067-14072.

(125) Gann, J. P.; Yan, M. Langmuir 2008, 24, 5319-5323.

(126) Yan, M. Polym. News 2002, 27, 6-12.

(127) Joester, D.; Klein, E.; Geiger, B.; Addadi, L. J. Am. Chem. Soc. 2006, 128, 1119-1124.

(128) Pei, Y.; Yu, H.; Pei, Z.; Theurer, M.; Ammer, C.; Andre, S.; Gabius, H. J.; Yan, M.; Ramstrom, O. Anal. Chem. 2007, 79, 6897-6902.

(129) Keana, J. F. W.; Cai, S. X. J. Org. Chem. 2002, 55, 3640-3647.

(130) Danehy, J. P.; Doherty, B. T.; Egan, C. P. J. Org. Chem. 2002, 36, 2525-2530.

(131) Pons, A.; Roca, P.; Aguiló, C.; Garcia, F.; Alemany, M.; Palou, A. J Biochem Biophys Methods. 1981, 4, 227-231.

(132) Trevelyan, W. E.; Forrest, R. S.; Harrison, J. S. Nature 1952, 170, 626-627.

(133) Chien, Y.; Jan, M.; Adak, A.; Tzeng, H.; Lin, Y.; Chen, Y.; Wang, K.; Chen, C.;

Chen, C.; Lin, C. ChemBioChem 2008, 9, 1100-1109.

(134) Anita, L.; Frank, G. L.; Clement, K. B. Eur. J. Biochem. 1980, 103, 307-312. 
(135) Gupta, D.; Dam, T. K.; Oscarson, S.; Brewer, C. F. J. Biol. Chem. 1997, 272, 6388-6392.

(136) Mandal, D. K.; Kishore, N.; Brewer, C. F. Biochemistry 1994, 33, 1149-1156.

(137) Grainger, D. W.; Castner, D. G. Adv. Mater. 2008, 20, 867-877.

(138) Hino, K.; Shingai, R.; Morita, T.; Toku, K.; Hirano, T.; Yoshikawa, H.; Nakano, H.; Nishi, N. Chem. Phys. Lett. 2008, 460, 173-177.

(139) Regev, O.; Backov, R.; Faure, C. Chem. Mater. 2004, 16, 5280-5285.

(140) Liu, L. H.; Dietsch, H.; Schurtenberger, P.; Yan, M. Bioconjugate Chem. 2009, 20, 1349-1355.

(141) Poveda, A.; Jimenez-Barbero, J. Chem. Soc. Rev. 1998, 27, 133-143.

(142) Smith, E. A.; Thomas, W. D.; Kiessling, L. L.; Corn, R. M. J. Am. Chem. Soc. 2003, 125, 6140-6148.

(143) Beccati, D.; Halkes, K. M.; Batema, G. D.; Guillena, G.; de Souza, A. C.; van Koten, G.; Kamerling, J. P. ChemBioChem 2005, 6, 1196-1203.

(144) von Itzstein, M.; Colman, P. Curr. Opin. Struct. Biol. 1996, 6, 703-709.

(145) Fernandez, M. D.; Canada, F. J.; Jimenez-Barbero, J.; Cuevas, G. J. Am. Chem. Soc. 2005, 127, 7379-7386.

(146) Clarke, C.; Woods, R. J.; Gluska, J.; Cooper, A.; Nutley, M. A.; Boons, G. J. J. Am. Chem. Soc. 2001, 123, 12238-12247.

(147) Lee, Y. C. J. Biochem. 1997, 121, 818-825.

(148) Sanders, J. N.; Chenoweth, S. A.; Schwarz, F. P. J. Inorg. Biochem. 1998, 70, 71-82.

(149) Liang, C.-H.; Wang, C.-C.; Lin, Y.-C.; Chen, C.-H.; Wong, C.-H.; Wu, C.-Y. Anal. Chem. 2009.

(150) Hone, D. C.; Haines, A. H.; Russell, D. A. Langmuir 2003, 19, 7141-7144.

(151) Lin, C.-C.; Yeh, Y.-C.; Yang, C.-Y.; Chen, C.-L.; Chen, G.-F.; Chen, C.-C.; Wu, Y.-C. J. Am. Chem. Soc. 2002, 124, 3508-3509.

(152) Mahon, E.; Aastrup, T.; Barboiu, M. Chem. Commun. 2010, 46, 5491-5493. 
(153) El-Boubbou, K.; Zhu, D. C.; Vasileiou, C.; Borhan, B.; Prosperi, D.; Li, W.; Huang, X. J. Am. Chem. Soc. 2010, 132, 4490-4499.

(154) Wang, X.; Ramström, O.; Yan, M. Adv. Mater. 2010, 22, 1946-1953.

(155) Wang, X.; Ramström, O.; Yan, M. Anal. Chem. 2010, 82, 9082-9089.

(156) Schärtl, W. Light Scattering from Polymer Solutions and Nanoparticle

Dispersions; Springer-Verlag: Berlin, Germany, 2007.

(157) Mishra, A.; Ram, S.; Ghosh, G. J. Phys. Chem. C 2009, 113, 6976-6982.

(158) Jans, H.; Liu, X.; Austin, L.; Maes, G.; Huo, Q. Anal. Chem. 2009, 81, 94259432.

(159) Falabella, J. B.; Cho, T. J.; Ripple, D. C.; Hackley, V. A.; Tarlov, M. J.

Langmuir 2010, 26, 12740-12747.

(160) Dai, Q.; Liu, X.; Coutts, J.; Austin, L.; Huo, Q. J. Am. Chem. Soc. 2008, 130, 8138-8139.

(161) Liu, X.; Dai, Q.; Austin, L.; Coutts, J.; Knowles, G.; Zou, J.; Chen, H.; Huo, Q. J. Am. Chem. Soc. 2008, 130, 2780-2782.

(162) Stöber, W.; Fink, A. J. Colloid Interface Sci. 1968, 26, 62-69.

(163) Chen, S.-L.; Dong, P.; Yang, G.-H. J. Colloid Interface Sci. 1997, 189, 268-272.

(164) Turkevich, J.; Stevenson, P. C.; Hollier, J. Discuss. Faraday Soc. 1951, 11, $55-$ 75.

(165) Wang, X.; Liu, L. H.; Ramstrom, O.; Yan, M. Exp. Biol. Med. 2009, 234, 11281139.

(166) Wang, X.; Ramstrom, O.; Yan, M. Chem. Commun. 2011, 47, 4261-4263.

(167) de la Fuente, J. M.; Penadés, S. Biochim. Biophys. Acta, Gen. Subj. 2006, 1760, 636-651.

(168) MacDougall, D.; Crummett, W. B. Anal. Chem. 1980, 52, 2242-2249.

(169) Long, G. L.; Winefordner, J. D. Anal. Chem. 1983, 55, 712A-724A.

(170) Sato, Y.; Murakami, T.; Yoshioka, K.; Niwa, O. Anal. Bioanal. Chem. 2008, $391,2527-2532$.

(171) Campbell, C. T.; Parker, S. C.; Starr, D. E. Science 2002, 298, 811-814. 
(172) De, M.; Miranda, O. R.; Rana, S.; Rotello, V. M. Chem. Commun. 2009, 21572159.

(173) Hill, H. D.; Millstone, J. E.; Banholzer, M. J.; Mirkin, C. A. ACS Nano 2009, 3, 418-424.

(174) Nyffenegger, R.; Quellet, C.; Ricka, J. J. Colloid Interface Sci. 1993, 159, 150 157.

(175) Wu, A. M.; Wu, J. H.; Singh, T.; Lai, L.-J.; Yang, Z.; Herp, A. Mol. Immunol. 2006, 43, 1700-1715.

(176) Sharma, S.; Bharadwaj, S.; Surolia, A.; Podder, S. K. Biochem. J 1998, 333, $539-542$.

(177) Schofield, C. L.; Mukhopadhyay, B.; Hardy, S. M.; McDonnell, M. B.; Field, R. A.; Russell, D. A. Analyst 2008, 133, 626-634.

(178) Koeller, K. M.; Wong, C. H. Nat. Biotechnol. 2000, 18, 835-841.

(179) Lewis, E. A.; Murphy, K. P. In Protein-Ligand Interactions: Methods and

Applications; Nienhaus, G. U., Ed.; Humana Press: Totowa, New Jersey, 2005, p 1-17.

(180) Wang, X.; Ramstrom, O.; Yan, M. Analyst 2011, 136, 4174-4178.

(181) Wang, X.; Ramström, O.; Yan, M. J. Mater. Chem. 2009, 19, 8944-8949.

(182) Shenhar, R.; Rotello, V. M. Acc. Chem. Res. 2003, 36, 549-561.

(183) Bittiger, H.; Schnebli, H. P. Concanavalin A as a Tool; John Wiley and Sons:

London, 1976.

(184) Barrientos, L. G.; Gronenborn, A. M. Mini-Rev. Med. Chem. 2005, 5, 21-31.

(185) Bewley, C. A.; Otero-Quintero, S. J. Am. Chem. Soc. 2001, 123, 3892-3902.

(186) Bolmstedt, A. J.; O'Keefe, B. R.; Shenoy, S. R.; Mcmahon, J. B.; Boyd, M. R.

Mol. Pharmacol. 2001, 59, 949-954.

(187) Shenoy, S. R.; Barrientos, L. G.; Ratner, D. M.; O'Keefe, B. R.; Seeberger, P. H.; Gronenborn, A. M.; Boyd, M. R. Chem. Biol. 2002, 9, 1109-1118.

(188) Matei, E.; Furey, W.; Gronenborn, A. M. Structure 2008, 16, 1183-1194.

(189) Wang, X.; Matei, E.; Deng, L. Q.; Ramstrom, O.; Gronenborn, A. M.; Yan, M.

Chem. Commun. 2011, 47, 8620-8622. 
(190) Dam, T. K.; Brewer, C. F. Glycobiology 2010, 20, 270-279.

(191) Drechsler, U.; Erdogan, B.; Rotello, V. M. Chem. Eur. J. 2004, 10, 5570-5579.

(192) Huskens, J. Curr. Opin. Chem. Biol. 2006, 10, 537-543.

(193) Jayaraman, N. Chem. Soc. Rev. 2009, 38, 3463-3483.

(194) Roy, D.; Fendler, J. Adv. Mater. 2004, 16, 479-508.

(195) Rao, C. N. R.; Biswas, K. Annu. Rev. Anal. Chem. 2009, 2, 435-462.

(196) Lyu, Y.-K.; Lim, K.-R.; Lee, B. Y.; Kim, K. S.; Lee, W.-Y. Chem. Commun. 2008, 4771-4773.

(197) Tsai, C.-S.; Yu, T.-B.; Chen, C.-T. Chem. Commun. 2005, 4273-4275.

(198) McKenzie, G. H.; Sawyer, W. H.; Nichol, L. W. Biochim. Biophys. Acta. 1972, 263, 283-293.

(199) Huet, M. Eur. J. Biochem. 1975, 59, 627-632.

(200) Kanai, M.; Mortell, K. H.; Kiessling, L. L. J. Am. Chem. Soc 1997, 119, 99319932.

(201) Cairo, C. W.; Gestwicki, J. E.; Kanai, M.; Kiessling, L. L. J. Am. Chem. Soc 2002, 124, 1615-1619.

(202) Jason, E. G.; Laura, E. S.; Laura, L. K. Angew. Chem. Int. Ed. 2000, 39, 45674570 .

(203) Barrientos, A. G.; Fuente, J. M. d. 1.; Jiménez, M.; Solís, D.; Cañada, F. J.;

Martín-Lomas, M.; Penadés, S. Carbohydrate Res. 2009, 344, 1474-1478.

(204) Pei, Z.; Yu, H.; Theurer, M.; Waldén, A.; Nilsson, P.; Yan, M.; Ramström, O. ChemBioChem 2007, 8, 166-168.

(205) Koehler, L. H. Anal. Chem. 1952, 24, 1576-1579.

(206) Cheng, Y.-C.; Prusoff, W. H. Biochem. Pharmacol. 1973, 22, 3099-3108.

(207) Mortell, K. H.; Weatherman, R. V.; Kiessling, L. L. J. Am. Chem. Soc 1996, 118 , 2297-2298.

(208) Wolfenden, M. L.; Cloninger, M. J. J. Am. Chem. Soc 2005, 127, 12168-12169.

(209) Schwarz, F. P.; Puri, K. D.; Bhat, R. G.; Surolia, A. J. Biol. Chem. 1993, 268, 7668-7677. 
(210) Dhayal, M.; Ratner, D. M. Langmuir 2009, 25, 2181-2187.

(211) Pepperkok, R.; Ellenberg, J. Nat. Rev. Mol. Cell Biol. 2006, 7, 690-696.

(212) Krutzik, P. O.; Nolan, G. P. Nat. Methods 2006, 3, 361-368.

(213) Kuno, A.; Uchiyama, N.; Koseki-Kuno, S.; Ebe, Y.; Takashima, S.; Yamada, M.;

Hirabayashi, J. Nat. Methods 2005, 2, 851-856.

(214) Van Blaaderen, A.; Vrij, A. Langmuir 1992, 8, 2921-2931.

(215) Yao, G.; Wang, L.; Wu, Y. R.; Smith, J.; Xu, J. S.; Zhao, W. J.; Lee, E. J.; Tan,

W. H. Anal. Bioanal. Chem. 2006, 385, 518-524.

(216) Ow, H.; Larson, D. R.; Srivastava, M.; Baird, B. A.; Webb, W. W.; Wiesner, U. Nano Lett. 2005, 5, 113-117.

(217) Larson, D. R.; Ow, H.; Vishwasrao, H. D.; Heikal, A. A.; Wiesner, U.; Webb, W. W. Chem. Mater. 2008, 20, 2677-2684.

(218) Zhao, X. J.; Bagwe, R. P.; Tan, W. H. Adv. Mater. 2004, 16, 173-176.

(219) Wang, L.; Wang, K. M.; Santra, S.; Zhao, X. J.; Hilliard, L. R.; Smith, J. E.; Wu, J. R.; Tan, W. H. Anal. Chem. 2006, 78, 646-654.

(220) Yan, M. Chem. Eur. J. 2007, 13, 4138-4144.

(221) Dwek, R. A. Chem. Rev. 1996, 96, 683-720.

(222) Varki, A. Glycobiology 1993, 3, 97-130.

(223) Sears, P.; Wong, C. H. Proc. Natl. Acad. Sci. U. S. A. 1996, 93, 12086-12093.

(224) MacBeath, G.; Schreiber, S. L. Science 2000, 289, 1760-1763.

(225) Tapec, R.; Zhao, X. J. J.; Tan, W. H. J. Nanosci. Nanotechnol. 2002, 2, 405-409.

(226) Harris, S. L.; Spears, P. A.; Havell, E. A.; Hamrick, T. S.; Horton, J. R.;

Orndorff, P. E. J. Bacteriol. 2001, 183, 4099-4102.

(227) Krogfelt, K. A.; Bergmans, H.; Klemm, P. Infect. Immun. 1990, 58, 1995-1998.

(228) Lian, W.; Litherland, S. A.; Badrane, H.; Tan, W. H.; Wu, D. H.; Baker, H. V.;

Gulig, P. A.; Lim, D. V.; Jin, S. G. Anal. Biochem. 2004, 334, 135-144.

(229) MacBeath, G. Nat. Genetics 2002, 32, 526 - 532.

(230) Vlacich, G.; Roe, C.; Webb, G. C. Nat. Clin. Pract. End. Met. 2007, 3, 594-605. 
(231) Khan, M. I.; Swamy, M. J.; Sastry, M. V. K.; Sajjan, S. U.; Patanjali, S. R.; Rao, P.; Swarnalatha, G. V.; Banerjee, P.; Surolia, A. Glycoconjugate J. 1988, 5, 75-84. (232) De Boeck, H.; Lis, H.; van Tilbeurgh, H.; Sharon, N.; Loontiens, F. G. J. Biol. Chem. 1984, 259, 7067-7074.

(233) Etzler, M.; Gupta, S.; Borrebaeck, C. J. Biol. Chem. 1981, 256, 2367-2370.

(234) Bains, G.; Lee, R. T.; Lee, Y. C.; Freire, E. Biochemistry 1992, 31, 12624-12628. (235) Shinohara, Y.; Kim, F.; Shimizu, M.; Goto, M.; Tosu, M.; Hasegawa, Y. Eur. J. Biochem. 1994, 223, 189-194.

(236) Tyagi, A.; Wang, X.; Deng, L. Q.; Ramström, O.; Yan, M. Biosens. Bioelectron. 2010, 26, 344-350.

(237) Wang, H.; Zhang, Y.; Yuan, X.; Chen, Y.; Yan, M. Bioconjugate Chem. 2010, 22, 26-32.

(238) Johnson, M. A.; Pinto, B. M. Carbohydr. Res. 2004, 339, 907-928.

(239) Gervay, J.; McReynolds, K. D. Curr. Med. Chem. 1996, 6, 129-153.

(240) Dam, T. K.; Brewer, C. F. Chem. Rev. 2002, 102, 387-430.

(241) Knibbs, R. N.; Agrwal, N.; Wang, J. L.; Goldstein, I. J. J. Biol. Chem. 1993, 268, 14940-14947.

(242) Cho, M.; Cummings, R. D. Biochemistry 1996, 35, 13081-13088.

(243) Polizzotti, B. D.; Maheshwari, R.; Vinkenborg, J.; Kiick, K. L. Macromolecules 2007, 40, 7103-7110.

(244) Deng, L.; Norberg, O.; Uppalapati, S.; Yan, M.; Ramstrom, O. Org. Biomol. Chem. 2011. (245) Norberg, O.; Deng, L.; Aastrup, T.; Yan, M.; Ramstrom, O. Anal. Chem. 2011, 83, 1000-1007.

(246) Norberg, O.; Deng, L.; Yan, M.; Ramstrom, O. Bioconjugate Chem. 2009, 20, 2364-2370. 


\section{Appendices}

Appendix A. List of Publications

Wang, X.; Ramström, O.; Yan, M., Thermodynamic analysis of interactions between glyco-nanoparticles and binding lectins via isothermal titration calorimetry. submitted for publication.

Wang, X.; Matei, E.; Deng, L. Q.; Ramström, O.; Gronenborn, A. M.; Yan, M., Interaction of fluorescent silica glyconanoparticles with lectin "supermicroarray": a high-throughput glycan screening strategy. submitted for publication.

Wang, X.; Ramström, O.; Yan, M., Dynamic Light Scattering as Efficient Tool to Study Glyconanoparticle-Lectin Interactions. Analyst 2011, 136, 41744178.

Wang, X.; Matei, E.; Deng, L. Q.; Ramström, O.; Gronenborn, A. M.; Yan, M., Multivalent Glyconanoparticles with Enhanced Affinity to Anti-Viral Lectin Cyanovirin-N Chem. Commun. 2011, 47, 8620-8622.

> Wang, X.; Ramström, O.; Yan, M., Dye-doped silica nanoparticles as efficient labels for glycans. Chem. Commun. 2011, 47 (14), 4261-4263.

> Wang, X.; Ramström, O.; Yan, M., Quantitative analysis of Multivalent Ligands Presentation on Gold Glyconanoparticles and Their Effects on Protein Binding. Anal. Chem. 2010, 82 (21), 9082-9089.

> Wang, X.; Ramström, O.; Yan, M., Glyconanomaterials: Synthesis, Characterization, and Ligand Presentation. Adv. Mater. 2010, 22, 1946-1953.

Wang, X.; Ramström, O.; Yan, M., A Photochemically Initiated Chemistry for Coupling Underivatized Carbohydrates to Gold Nanoparticles. J. Mater. Chem. 2009, 19 (47), 8944-8949. 
Dang, X.; Liu, L. H.; Ramström, O.; Yan, M. D., Engineering Nanomaterial Surfaces for Biomedical Applications. Exp. Biol. Med. 2009, 234 (10), 11281139.

\section{Papers not included in this dissertation:}

$>$ Deng, L. Q.; Wang, X.; Norberg, O.; Dong, H.; Joliton, A.; Yan, M.; Ramstrom, O., Stereocontrolled 1-S-Glycosylation and Comparative Binding Studies of Photoprobe-Thiosaccharide Conjugates with Their O-Linked Analogs. submitted for publication.

$>$ Tong, Q.; Wang, X.; Wang, H.; Yan, M., Glyco-nanoparticle microarray for detection of carbohydrate-protein interactions. submitted for publication.

> Wang, X. H.; Wang, X.; Fernández, R.; Yan, M.; La Rosa, A., Nanolithography on Responsive Surfaces. J. Nanoscience Lett. 2011, in press.

> Kubo, T.; Wang, X.; Tong, Q.; Yan, M., Polymer-Based Photocoupling Agent for the Efficient Immobilization of Small Molecules and Nanomaterials Langmuir 2011, 27, 9372-9378.

> Wang, X. H.; Wang, X.; Fernández, R.; Ocola, L.; Yan, M.; La Rosa, A., Electric Field-Assisted Dip-pen Nanolithography on poly(4-vinylpyridine) (P4VP) Thin Films. ACS Appl. Mater. Interfaces 2010, 2 (10), 2904-2909.

> Tyagi, A.; Wang, X.; Deng, L. Q.; Ramström, O.; Yan, M., Photogenerated Carbohydrate Microarrays to Study Carbohydrate-Protein Interactions using Surface Plasmon Resonance Imaging. Biosens. Bioelectron. 2010, 26, 344-350.

> Surangi, J.; Wang, X.; Yan, M., Linear Discriminant Analysis and Statistical Pattern Lectin Recognition Using Gold Glyconanoparticle. in preparation.

Zorn, G.; Tyagi, A.; Wang, X.; Wang, H.; Castner, D. G.; Yan, M., Chemical Composition and Presentation of Perfluorophenylazide Surfaces: Analysis and Impact on Ligand Immobilization. in preparation. 


\section{Appendix B. Copyright Information}

1. Permission for Wang, X.; Ramström, O.; Yan, M. Anal. Chem. 2010, 82 (21), 9082-

9089

Copyright

Clearance

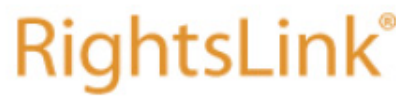

Home

ACS Publications Title: High quality High impact.
Quantitative Analysis of

Multivalent Ligand Presentation on Gold Glyconanoparticles and the Impact on Lectin Binding

\section{Author:}

Xin Wang et al.

Publication: Analytical Chemistry

Publisher: American Chemical Society

Date: Nov 1, 2010

Copyright (c) 2010, American Chemical Society
Logged in as:

Xin Wang

Account \#:

3000429954

LOGOUT

\section{Quick Price Estimate}

Permission for this particular request is granted for print and electronic formats at no charge. Figures and tables may be modified. Appropriate credit should be given. Please print this page for your records and provide a copy to your publisher. Requests for up to 4 figures require only this record. Five or more figures will generate a printout of additional terms and conditions. Appropriate credit should read: "Reprinted with permission from \{COMPLETE REFERENCE CITATION\}. Copyright \{YEAR\} American Chemical Society." Insert appropriate information in place of the capitalized words.

I would like to...? reuse in a Thesis/Dissertation

Requestor Type?

Portion 3

Format 3

Will you be

translating?

Select your currency

Quick Price
Author (original work)

Full article

Electronic

No

USD - \$

Click Quick Price

\section{QUICK PRICE}

\section{CONTINUE}

To request permission for a type of use not listed, please contact the publisher directly.

This service provides permission for reuse only. If you do not have a copy of the article you are using, you may copy and paste the content and reuse according to the terms of your agreement. Please be advised that obtaining the content you license is a separate transaction not involving Rightslink. 
2. Permission for Wang, X.; Ramström, O.; Yan, M. Adv. Mater. 2010, 22, 1946-1953

\section{JOHN WILEY AND SONS LICENSE \\ TERMS AND CONDITIONS}

Oct 26,2011

This is a License Agreement between Xin Wang ("You") and John Wiley and Sons ("John Wiley and Sons") provided by Copyright Clearance Center ("CCC"). The license consists of your order details, the terms and conditions provided by John Wiley and Sons, and the payment terms and conditions.

\begin{tabular}{|c|c|}
\hline \multicolumn{2}{|c|}{$\begin{array}{l}\text { All payments must be made in full to CCC. For payment instructions, please see } \\
\text { information listed at the bottom of this form. }\end{array}$} \\
\hline License Number & 2776291059464 \\
\hline License date & Oct 26, 2011 \\
\hline Licensed content publisher & John Wiley and Sons \\
\hline Licensed content publication & Advanced Materials \\
\hline Licensed content title & $\begin{array}{l}\text { Glyconanomaterials: Synthesis, Characterization, and Ligand } \\
\text { Presentation }\end{array}$ \\
\hline Licensed content author & Xin Wang,Olof Ramström,Mingdi Yan \\
\hline Licensed content date & May 4, 2010 \\
\hline Start page & 1946 \\
\hline End page & 1953 \\
\hline Type of use & Dissertation/Thesis \\
\hline Requestor type & Author of this Wiley article \\
\hline Format & Electronic \\
\hline Portion & Full article \\
\hline Will you be translating? & No \\
\hline \multicolumn{2}{|l|}{ Order reference number } \\
\hline Total & 0.00 USD \\
\hline
\end{tabular}




\section{Permission for Wang, X.; Liu, L. H.; Ramström, O.; Yan, M. D. Exp. Biol. Med.}

2009, 234 (10), 1128-1139

\section{http://www.rsmjournals.com/site/misc/reprints.xhtml}

Reprints and Permissions

Corporate Reprints

For more information about Corporate Reprints, please visit Corporate Services.

\section{Author Reprints}

If you are the author of an article, you may order reprints at a special author rate. Please contact the Reprints team for $n$ information.

Author reprints must be used solely for the author's personal use. For other uses, see Corporate Services.

\section{Single Copies of articles}

Single PDF copies of articles may be downloaded from the joumal Websites.

Subscribers may download single copies of current articles free of charge.

Nonsubscribers may download single copies of articles via the pay-per-view option. For details, simply go to the article download. If you are not a subscriber, ordering information will appear.

Permissions for Authors

Authors do not need to obtain permission for the following:

1. to use their original figures or tables in their future works;

2. to make copies of their papers for their own personal use, including classroom use, or for the personal use colleagues, provided those copies are not for sale and are not distributed in a systematic way;

3. to include their papers as part of their dissertations;

4. to use all or part of their article in a printed compilation of their own works.

Citation of the original source must be included and copies must include the copyright notice of the original publication.

Other permissions

Anyone may, without requesting permission, use original figures or tables published in RSM Press joumals for noncomr educational use (e.g. in a review article) provided that the original source and copyright notice are cited.

For permission to reprint material for commercial purposes, requests must be sent via e-mail to the Permissions team. The email should include the following information about the original material:

1. Your full name, affiliation, and title

2. Your complete mailing address, phone number, fax number, and e-mail address

3. The full citation of the article from which you wish to reproduce material: author(s) name(s), title, year of publication, volume number and page range

4. Figure/table number or portion of text to be reprinted

The email should also include the following information about the intended use of the material:

1. Title of work in which material will appear

2. Authors/editors of work

3. Publisher of work

4. Retail price of work

5. Number of copies of work to be produced

6. Whether work is for nonprofit or commercial use

RSM Press cannot supply original artwork. Use of material must not imply any endorsement by RSM Press. The full jou reference must be cited and "Copyright (copyright year) Royal Society of Medicine Press, UK". 
4. Permission for (a) Wang, X.; Ramström, O.; Yan, M. J. Mater. Chem. 2009, 19 (47), 8944-8949; (b) Wang, X.; Ramström, O.; Yan, M. Chem. Commun. 2011, 47 (14), 4261-4263; (c) Wang, X.; Matei, E.; Deng, L. Q.; Ramström, O.; Gronenborn, A. M.; Yan, M., Chem. Commun. 2011, 47, 8620-8622; (d) Wang, X.; Ramström, O.; Yan, M. , Analyst 2011, 136, 4174-4178.

CONTRACTS-COPYRIGHT (shared) <Contracts-Copyright@rsc.org>

To: "xinw@pdx.edu" <xinw@pdx.edu>

Mon, Oct 31, 2011 at 3:16

Dear Xin Wang

The Royal Society of Chemistry (RSC) hereby grants permission for the use of your paper(s) specified below in the printed and microfilm version of your thesis. You may also make available the PDF version of your paper(s) that the RSC sent to the corresponding author(s) of your paper(s) upon publication of the paper(s) in the following ways: in your thesis via any website that your university may have for the deposition of theses, via you university's Intranet or via your own personal website. We are however unable to grant you permission to include the PDF version of the paper(s) on its own in your institutional repository. The Royal Society of Chemistry is a signatory to the STM Guidelines on Permissions (available on request).

Please note that if the material specified below or any part of it appears with credit or acknowledgement to a third party then you must also secure permission from that third party before reproducing that material.

Please ensure that the thesis states the following:

Reproduced by permission of The Royal Society of Chemistry

and include a link to the paper on the Royal Society of Chemistry's website

Please ensure that your co-authors are aware that you are including the paper in your thesis.

Regards

Gill Cockhead

Publishing Contracts \& Copyright Executive

Gill Cockhead (Mrs), Publishing Contracts \& Copyright Executive

Royal Society of Chemistry, Thomas Graham House

Science Park, Milton Road, Cambridge CB4 OWF, UK

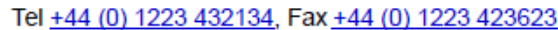

http://www.rsc.org 